Universidad Politécnica de Madrid

E. T. S. de Ingenieros en Topografía, Geodesia y Cartografía

Departamento de Ingeniería Topográfica y Cartografía

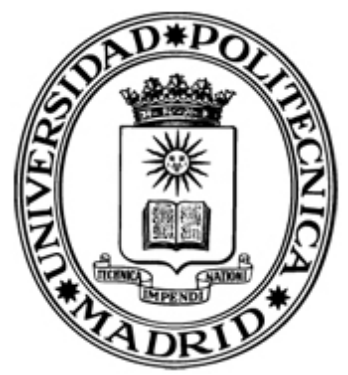

\title{
GEOGRAFÍA DEL CARBONO EN ALTA RESOLUCIÓN EN BOSQUE TROPICAL AMAZÓNICO DEL ECUADOR MEDIANTE SENSORES AEROTRANSPORTADOS
}

\section{TESIS DOCTORAL}

\author{
Autor: \\ Patricio Xavier Molina Simbaña \\ Director: Mercedes Farjas Abadia \\ Codirector: Juan Carlos Ojeda Manrique
}

MADRID

2016 
Tribunal nombrado por el Sr. Rector Magnífico de la Universidad Politécnica de Madrid, el día ... de ... de 2016.

Presidente:

Secretario:

Vocales:

Suplentes:

Realizado el acto de defensa y lectura de la Tesis el día ... de de 2016 en la E.T.S.I. en Topografía, Geodesia y Cartografía de la Universidad Politécnica de Madrid 


\section{RESUMEN}

La estimación de la biomasa de la vegetación terrestre en bosque tropical no sólo es un área de investigación en rápida expansión, sino también es un tema de gran interés para reducir las emisiones de carbono asociadas a la deforestación y la degradación forestal (REDD+). Las estimaciones de densidad de carbono sobre el suelo (ACD) en base a inventarios de campo y datos provenientes de sensores aerotransportados, en especial con sensores LiDAR, han conducido a un progreso sustancial en el cartografiado a gran escala de las reservas de carbono forestal. Sin embargo, estos mapas de carbono tienen incertidumbres considerables, asociadas generalmente al proceso de calibración del modelo de regresión utilizado para producir los mapas.

En esta tesis se establece una metodología para la calibración y validación de un modelo general de estimación de ACD usando LiDAR en un sector del Parque Nacional Yasuní en Ecuador. En el proceso de calibración del modelo se considera el tamaño y la ubicación de las parcelas, la influencia de la topografía y la distribución espacial de la biomasa. Para el análisis de los datos se utilizan técnicas geoestadísticas en combinación con variables geomorfométricas derivadas de datos LiDAR, y se propone un esquema de muestreo estratificado por posiciones topográficas (valle, ladera y cima). La validación del modelo general para toda la zona de estudio presentó valores de $\mathrm{RMSE}=5.81 \mathrm{Mg} \mathrm{C}^{-1}, \mathrm{R}^{2}=0.94$ y sesgo $=0.59$, mientras que, al considerar las posiciones topográficas, el modelo presentó valores de $\mathrm{RMSE}=1.67 \mathrm{Mg} \mathrm{C} \mathrm{ha}^{-1}, \mathrm{R}^{2}=0.98$ y sesgo $=0.23$ para el valle; $\mathrm{RMSE}=3.13$ $\mathrm{MgC} \mathrm{ha}{ }^{-1}, \mathrm{R}^{2}=0.98$ y sesgo $=-0.34$ para la ladera; $\mathrm{y} \mathrm{RMSE}=2.33 \mathrm{Mg} \mathrm{C} \mathrm{ha}^{-1}, \mathrm{R}^{2}=0.97 \mathrm{y}$ sesgo $=0.74$ para la cima.

Los resultados obtenidos demuestran que la metodología de muestreo estratificado por posiciones topográficas propuesto, permite calibrar de manera efectiva el modelo general con las estimaciones de ACD en campo, logrando reducir el RMSE y el sesgo. Los resultados muestran el potencial de los datos LiDAR para caracterizar la estructura vertical de la vegetación en un bosque altamente diverso, permitiendo realizar estimaciones precisas de ACD, y conocer patrones espaciales continuos de la distribución de la biomasa aérea y del contenido de carbono en la zona de estudio.

Palabras clave: Densidad de carbono forestal, LiDAR, análisis geoestadistico, muestreo estratificado, bosque tropical 


\begin{abstract}
Estimating biomass of terrestrial vegetation in tropical forest is not only a rapidly expanding research area, but also a subject of tremendous interest for reducing carbon emissions associated with deforestation and forest degradation (REDD+). The aboveground carbon density estimates (ACD) based on field inventories and airborne sensors, especially LiDAR sensors have led to a substantial progress in large-scale mapping of forest carbon stocks. However, these carbon maps have considerable uncertainties generally associated with the calibration of the regression model used to produce these maps.
\end{abstract}

This thesis establishes a methodology for calibrating and validating a general ACD estimation model using LiDAR in Ecuador's Yasuní National Park. The size and location of the plots are considered in the model calibration phase as well as the influence of topography and spatial distribution of biomass. Geostatistical analysis techniques are used in combination with geomorphometrics variables derived from LiDAR data, and then a stratified sampling scheme considering topographic positions (valley, slope and ridge) is proposed. The validation of the general model for the study area showed values of RMSE = $5.81 \mathrm{Mg} \mathrm{C} \mathrm{ha}^{-1}, \mathrm{R}^{2}=0.94$ and bias $=0.59$, while considering the topographical positions, the model showed values of $\mathrm{RMSE}=1.67 \mathrm{Mg} \mathrm{C} \mathrm{ha}^{-1}, \mathrm{R}^{2}=0.98$ and bias $=0.23$ for the valley; $\mathrm{RMSE}=3.13 \mathrm{Mg} \mathrm{C}$ ha- $1, \mathrm{R}^{2}=0.98$ and bias = -0.34 for the slope; and $\mathrm{RMSE}=$ $2.33 \mathrm{Mg} \mathrm{Cha}^{-1}, \mathrm{R}^{2}=0.97$ and bias $=0.74$ for the ridge.

The results show that the stratified sampling methodology taking into account topographic positions, effectively calibrates the general model with field estimates of ACD, reducing RMSE and bias. The results show the potential of LiDAR data to characterize the vertical structure of vegetation in a highly diverse forest, allowing accurate estimates of $\mathrm{ACD}$, and knowing continuous spatial patterns of biomass distribution and carbon stocks in the study area.

Keywords: forest carbon density, LiDAR, geostatistical analysis, stratified sampling, tropical forest. 
A mi familia, por su apoyo incondicional. A Xavier Israel y María Fernanda, por su paciencia y comprensión durante el tiempo que estuve ausente de casa. 


\section{AGRADECIMIENTOS}

No quiero dejar pasar la oportunidad para expresar mi más profundo y sincero agradecimiento a las personas y organizaciones que han colaborado directa o indirectamente en la realización y consecución de este trabajo.

A la Doctora Mercedes Farjas Abadía y al Doctor Juan Carlos Ojeda Manrique, Director y Codirector de tesis respectivamente, por sus acertadas orientaciones, seguimiento y asesoramiento en todas las etapas de la investigación. Al Doctor Gregory P. Asner y a los miembros del Carnegie Airborne Observatory en la Universidad de Stanford, por el apoyo y tiempo dedicado durante mi estancia de investigación y a lo largo de toda la investigación. Al Doctor Renato Valencia, de la Estación Científica Yasuní, por su colaboración permanente en los temas relacionados con la ecología tropical. Al Doctor Luis Alberto Sánchez Diez por su asistencia con los temas estadísticos. Al Doctor Antonio Vázquez Hoehne por su ayuda con los aspectos administrativos en la Escuela. Al Mayor de Ingeniería del Ejército Ecuatoriano Geovanny Vergara, por su apoyo durante la fase de captura de datos LiDAR. A mis compañeros doctorandos del Centro GEO I+D, Adolfo, Alberto, Ayar, Eladio, Víctor y Vladimir por los gratos momentos compartidos durante los últimos años.

Al Director y Subdirector del Instituto Geográfico Militar de Ecuador por el apoyo logístico y técnico durante las largas jornadas de trabajo en el Parque Nacional Yasuní. A la Secretaria de Educación Superior, Ciencia, Tecnología e Innovación del Ecuador por la beca otorgada para cursar los estudios de Doctorado en la UPM, y al Ejército Ecuatoriano por la autorización para ausentarme del país y continuar con los estudios de Doctorado.

Por último y, sobre todo, el agradecimiento más profundo y sentido para mi familia, y a una persona en especial, Charo. Sin su apoyo, paciencia e inspiración habría sido imposible llevar a cabo esta dura tarea.

Aquí se termina mi tesis, y con ella una etapa difícil y a la vez apasionante de mi vida, que marca el inicio de otra llena de expectativas y nuevos retos.

Misión cumplida, es hora de recoger mis pertrechos y volver a casa.

"La gratitud no es solo la mayor de las virtudes, sino la madre de todas las demás."

Cicerón 


\section{TABLA DE CONTENIDOS}

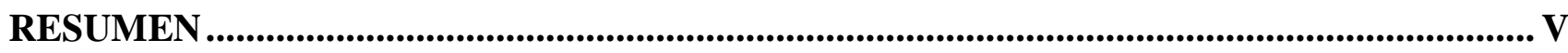

LISTADO DE FIGURAS …….........................................................................................................XVI

LISTADO DE TABLAS ……..............................................................................................................XIX

LISTADO DE ACRÓNIMOS Y SIGLAS ....................................................................................XXI

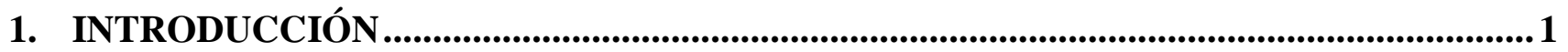

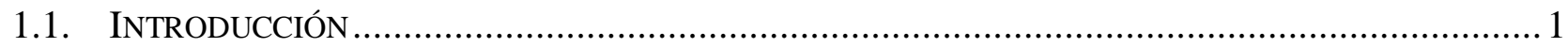

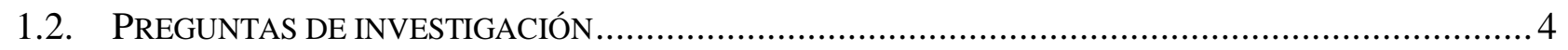

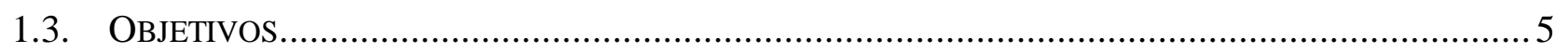

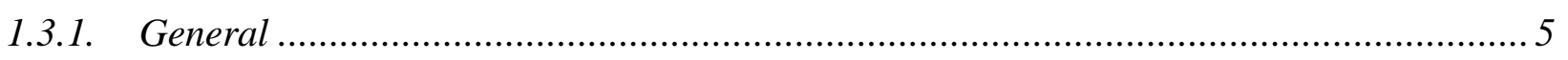

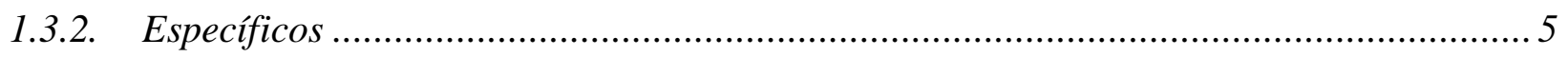

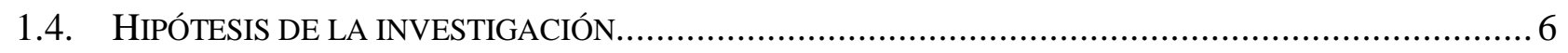

1.5. ESTRUCTURA DE LA TESIS Y METODOLOGÍA UTILIZADA ....................................................... 6

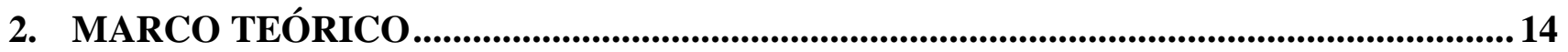

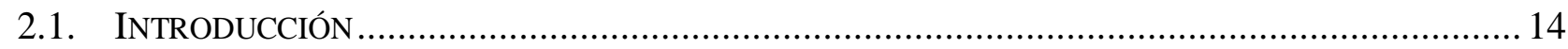

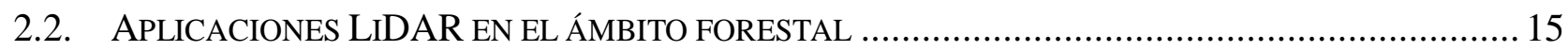

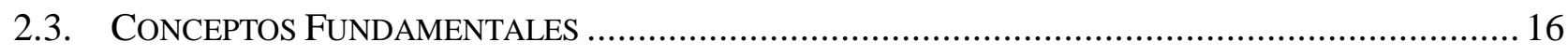

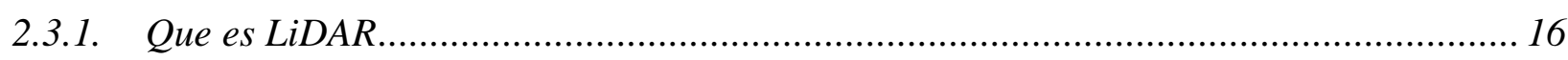

2.3.2. Estructura tridimensional de la vegetación forestal .................................................. 19

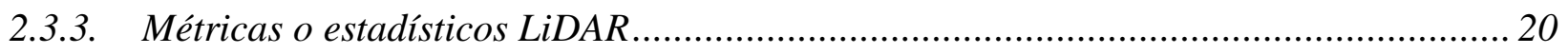

2.4. PRINCIPALES VARIABLES Y PARÁMETROS DE ESTRUCTURA FORESTAL INFERIDOS DE DATOS

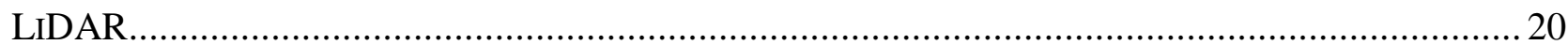

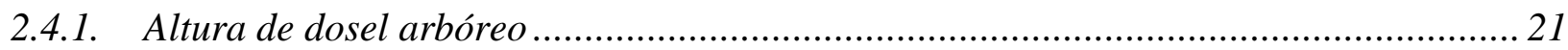

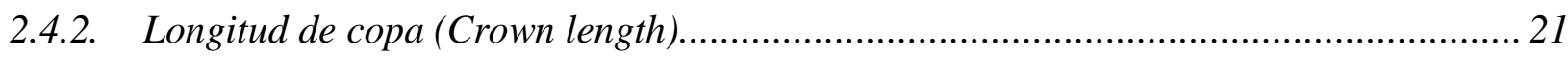

2.4.3. Diámetro a la altura de pecho y área basimétrica......................................................... 22

2.4.4. Fracción de cabida cubierta (fractional canopy cover)................................................ 22

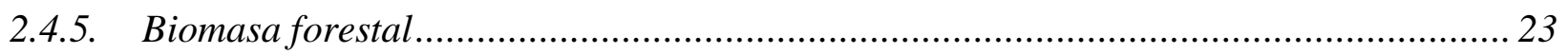




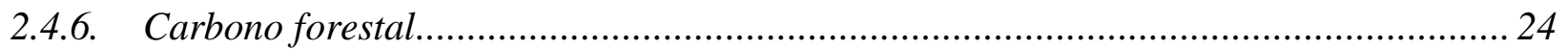

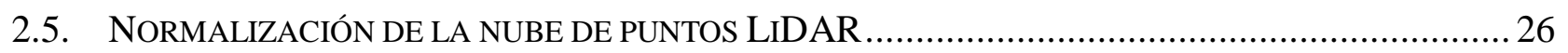

2.5.1. Clasificación o filtrado de puntos LiDAR ................................................................ 26

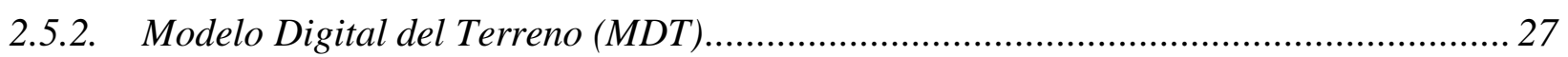

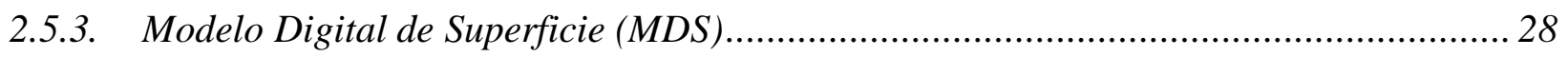

2.6. ESTIMACIÓN DE BIOMASA SOBRE EL SUELO USANDO LIDAR ......................................... 28

2.6.1. Ecuaciones alométricas para estimar AGB en bosques tropicales............................. 30

2.6.2. Escalamiento alométrico de parcelas agregadas ..................................................... 31

2.6.3. Modelo general de estimación de ACD ..................................................................... 33

2.6.4. Fuentes de error en las estimaciones de ACD usando LiDAR ..................................... 34

2.6.5. Influencia de la topografía y del tamaño de parcela.................................................. 35

2.7. MÉTOdOS DE ESTIMACIÓN DE VARIABLES FORESTALES CON LIDAR ................................... 37

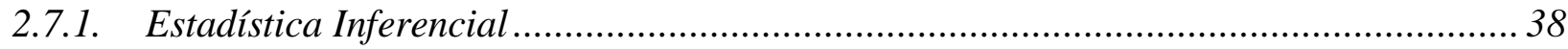

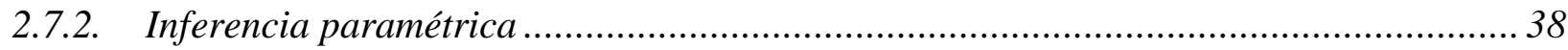

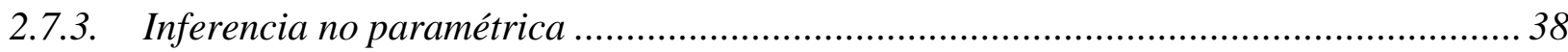

2.8. EL MODELO DE REGRESIÓN LINEAL MÚLTIPLE .............................................................. 38

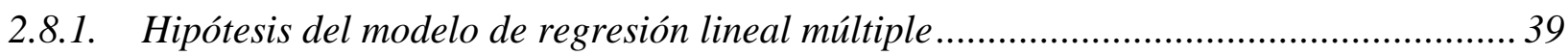

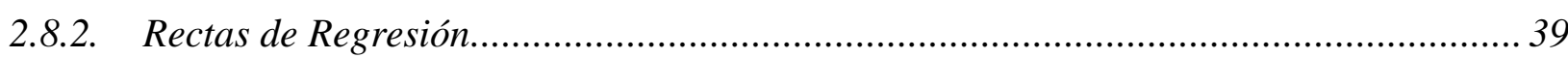

2.8.3. Modelos de regresión utilizados para inventario con LiDAR .................................... 40

2.9. COMPARACIÓN, SELECCIÓN Y VALIDACIÓN DE MODELOS ................................................... 42

2.9.1. Criterios de selección de modelos .............................................................................. 42

2.9.2. Ajuste de modelos de regresión lineal múltiples...................................................... 44

3. ESTUDIO DE CASO: PARQUE NACIONAL YASUNÍ ..........................................5

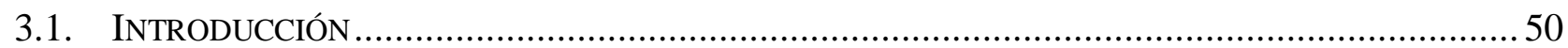

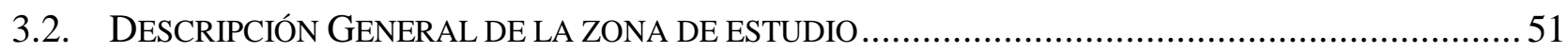

3.2.1. Descripción del Sector Napo-Curaray ................................................................ 52

3.2.2. Descripción del ecosistema Bosque siempreverde de tierras bajas del Napo-Curaray. 54

3.2.3. Descripción del ecosistema Bosque inundado de la llanura aluvial de la Amazonía..... 55

3.2.4. Parcela de investigación de la Pontificia Universidad Católica del Ecuador ............... 56

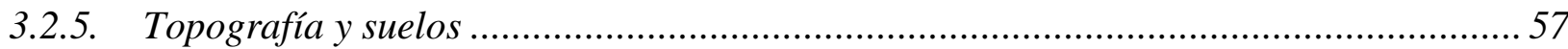

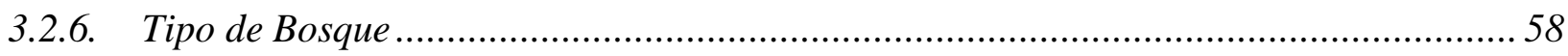

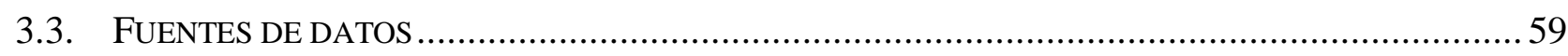

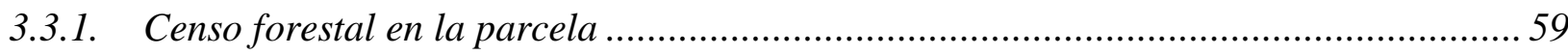


3.3.2. Determinación del tamaño de muestra para el cálculo de la biomasa. 59

3.3.3. Estimación de biomasa sobre el suelo usando modelos generales pantropicales .......... 61

3.3.4. Estimación del área basal en las parcelas ................................................................. 63

3.3.5. Geoposicionamiento y levantamiento planimétrico de la parcela ................................ 63

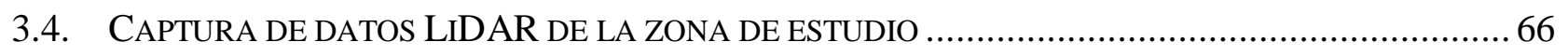

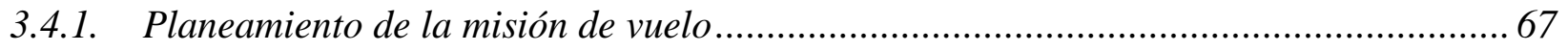

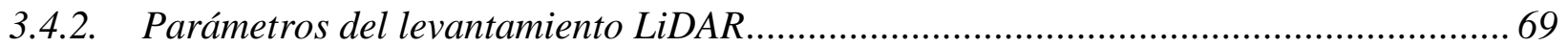

3.4.3. Planificación de la estación de referencia en tierra ................................................... 70

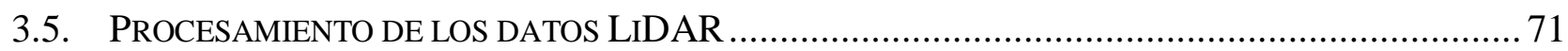

3.5.1. Generación del Modelo Digital del Terreno (MDT) .............................................. 72

3.5.2. Generación del Modelo Digital de Superficie normalizado (nMDS)........................... 73

3.5.3. Cálculo de la altura media al tope del dosel (TCH) y altura media del perfil de dosel (MCH) 75

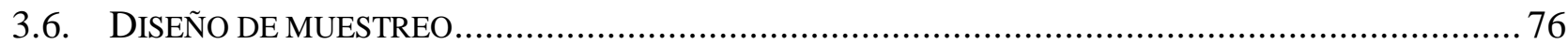

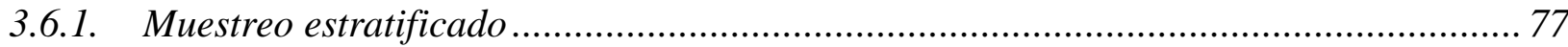

3.6.2. Hábitats topográficos en la zona de estudio................................................................ 77

3.6.3. Determinación de posiciones topográficas mediante análisis geo-estadístico............... 79

3.6.4. Estratificación por posiciones topográficas ........................................................... 79

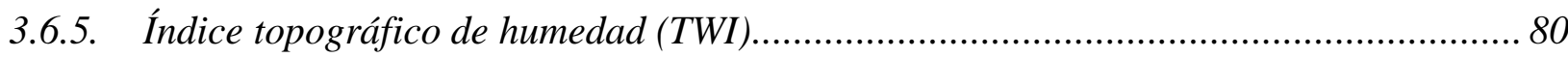

3.7. ANÁLISIS DE LA DISTRIBUCIÓN DE AGB EN LA PARCELA DE ESTUDIO ................................83

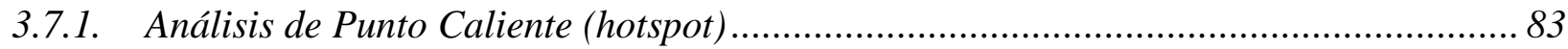

3.7.2. Consideraciones del análisis de punto caliente (hotspot) ........................................ 84

3.7.3. Cálculo de la Autocorrelación espacial en la parcela ............................................... 85

3.8. MODELO DE REGRESIÓN LINEAL PARA LA ESTIMACIÓN DE LA ACD .................................... 87

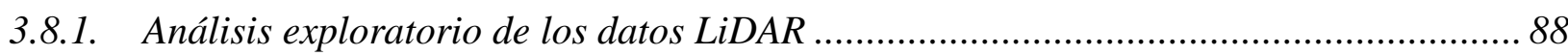

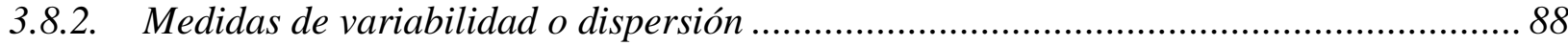

3.8.3. Condiciones previas para utilizar regresión lineal múltiple ...................................... 90

3.8.4. Análisis de correlación lineal de los datos .............................................................. 91

3.9. APLICACIÓN DEL MODELO GENERAL DE ESTIMACIÓN DE ACD .......................................... 93

3.9.1. Selección de las subparcelas para el ajuste y validación del modelo general............... 93

3.9.2. Ajuste del modelo .................................................................................................... 94

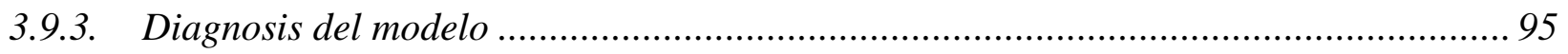

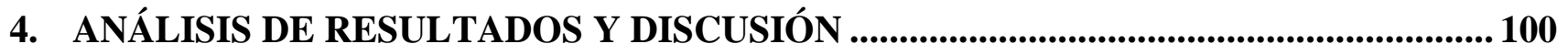




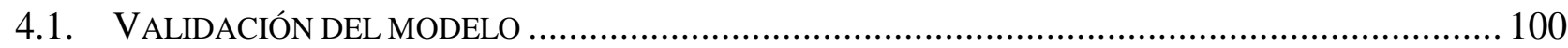

4.2. VALIDACIÓN DEL MODELO POR POSICIONES TOPOGRÁFICAS ............................................ 101

4.3. PRECISIÓN DE LAS ESTIMACIONES DE ACD CON EL MODELO LOCAL ................................... 102

4.4. MODELO PARA LA ESTIMACIÓN DE ÁREA BASAL A PARTIR DE DATOS LIDAR ...................... 104

4.5. CÁlCUlO DE LA DENSIDAD DE MADERA A PARTIR DE DATOS LIDAR ............................... 105

4.6. APLICACIÓN DEL MODELO GENERAL DE ESTIMACIÓN DE ACD EN OTRAS ZONAS DE ESTUDIO106

4.7. MODELO FINAL PARA LA ESTIMACIÓN DE ACD EN LA ZONA DE COBERTURA LiDAR ............ 106

4.8. GEOGRAFÍA DEL CARBONO EN LA ZONA DE ESTUDIO ...................................................... 106

4.9. ANÁLISIS DE LA INCERTIDUMBRE DEL MAPA DE CARBONO............................................... 108

4.9.1. Errores posicionales entre las parcelas de campo y los datos LiDAR ...................... 109

4.9.2. Diferencias temporales entre las mediciones LiDAR y las mediciones de campo........ 109

4.9.3. Discrepancias entre las mediciones de campo y las mediciones con LiDAR.............. 110

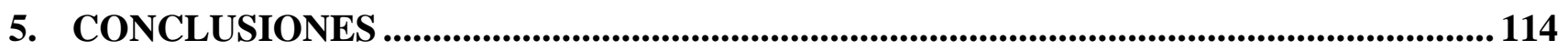

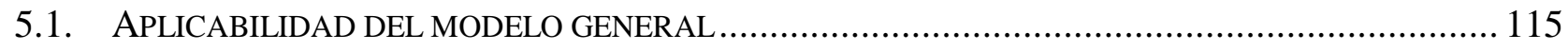

5.2. MUESTREO ESTRATIFICADO POR POSICIONES TOPOGRÁFICAS.......................................... 115

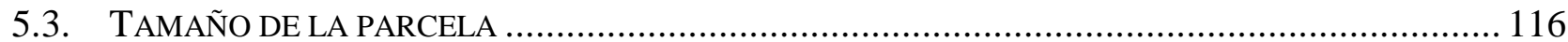

5.4. MAPA DE DENSIDAD DE CARBONO SOBRE EL SUELO ...................................................... 117

5.5. BENEFICIOS DEL CARTOGRAFIADO DE ACD DE ALTA PRECISIÓN ....................................... 117

5.6. APORTACIONES ORIGINALES DE LA INVESTIGACIÓN..................................................... 118

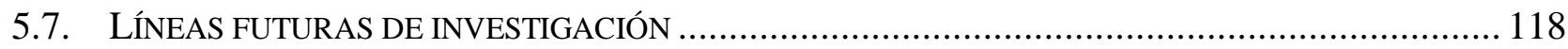

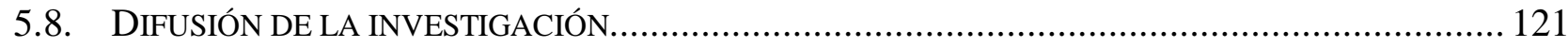

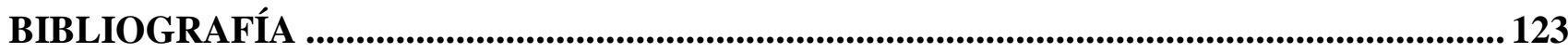

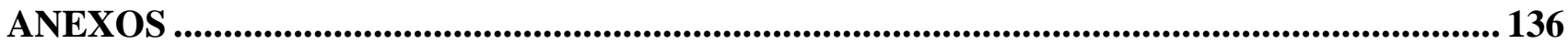

ANEXO A: LÍNEA DE TIEMPO DE LOS CENSOS REALIZADOS EN LA PARCELA DE 50 HA ............... 138

ANEXO B: CUADRO-RESUMEN DE LAS ESTIMACIONES DE LAS VARIABLES FORESTALES ........... 142

ANEXO C: MODELO DIGITAL DE SUPERFICIE NORMALIZADO DE CADA SUBPARCELA ................ 146

ANEXO D: RESUMEN DE LAS ESTADÍSTICAS DESCRIPTIVAS DEL TCH Y MCH DE LAS SUBPARCELAS

ANEXO E: ANÁLISIS EXPLORATORIO DE LOS DATOS LIDAR DE LAS SUBPARCELAS DE ESTUDIO 160 ANEXO F: MAPA DE ALTURA MEDIA AL TOPE DEL DOSEL DE LA ZONA DE ESTUDIO .................. 168 ANEXO G: MAPA DE LAS RESERVAS DE CARBONO SOBRE EL SUELO EN LA ZONA DE ESTUDIO ... 172 ANEXO H: CERTIFICADOS DE LAS ESTANCIAS DE INVESTIGACIÓN 


\section{LISTADO DE FIGURAS}

Figura 1.1: Unidades metodológicas de la investigación ............................................. 8

Figura 2.1: Componentes del sistema de datos LiDAR aerotransportado......................... 17

Figura 2.2: Nube de puntos LiDAR multi-retorno de un sector del bosque tropical en la

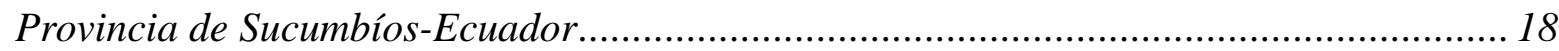

Figura 2.3: Esquema de múltiples retornos del pulso de luz láser en bosque tropical........ 18

Figura 2.4: Fotografía del dosel forestal en la zona de estudio........................................ 19

Figura 2.5: Concepto de voxel en el análisis de la estructura vertical del bosque. ............ 20

Figura 2.6: Distribución de retornos LiDAR de un árbol individual y el suelo y sus

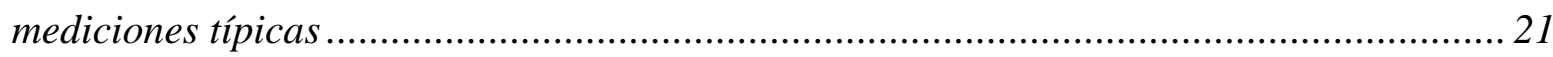

Figura 2.7: Esquema del proceso de cálculo de la cobertura de copas............................. 23

Figura 2.8: La proporción relativa de carbono (C), Agua (H2O), y otros elementos contenida en la biomasa. 24

Figura 2.9: Flujo de trabajo para la caracterización de variables forestales con un enfoque basado en métricas obtenidas con LiDAR.

Figura 3.1: Delimitación de la cuenca amazónica y ubicación del Parque Nacional "Yasunî" 51

Figura 3.2: Modelo digital del Terreno del sector Napo-Curaray .................................. 53

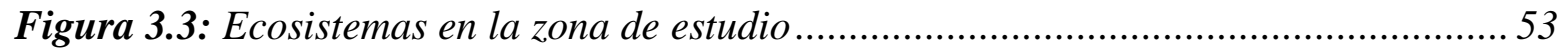

Figura 3.4: Ubicación de la parcela de investigación de 50 hectáreas............................... 56

Figura 3.5: Mapa de sombras de la parcela de estudio generado a partir de datos LiDAR.

Figura 3.6: MDT con la nube de puntos LiDAR con color RGB de la ortofotografía......... 59

Figura 3.7: Sistema de cuadriculado regular de subparcelas de 1 ha, utilizado para el cálculo de la biomasa sobre el suelo 61

Figura 3.8: Ubicación de los 4 puntos GNSS y las 4 esquinas de la parcela ..................... 64

Figura: 3.9: Levantamiento planimétrico de la parcela de estudio. 66

Figura 3.10: Esquema del flujo de trabajo para la generación de MDE (MDT, MDS) a 
partir de datos LiDAR.

Figura 3.11: Estrato bajo herbáceo y troncos caídos; trepadoras y lianas en la zona de estudio. 68

Figura 3.12: Avioneta CESSNA 172 Skyhawk y sensor LiDAR Optech ALTM Gemini ...... 69

Figura 3.13: Ventana del Plan de vuelo realizado en ALTM-NAV................................... 70

Figura 3.14: Trayectoria GPS de la misión LiDAR en la zona de estudio......................... 71

Figura 3.15: Modelo Digital del Terreno de la trayectoria de vuelo LiDAR ..................... 73

Figura 3.16: Modelo Digital de Superficie normalizado (nMDS) de toda la trayectoria de vuelo LiDAR...... 74

Figura 3.17: Modelo Digital de Superficie normalizado en 3D de la parcela de estudio. .. 74 Figura 3.18: Vista superior de la nube de puntos LiDAR de las parcelas $N^{o} 31$ y $N^{o} 36$ y perfil vertical de vegetación .75

Figura 3.19: Comparación de los coeficientes de variación del TCH y MCH para cada una de las subparcelas. 76

Figura 3.20: Hábitats topográficos en la zona de estudio. 78

Figura 3.21: Perfil longitudinal y transversal de la parcela de estudio con la nube de puntos LiDAR visualizada con color RGB transferidos de la ortofotografía. .78

Figura 3.22: Índice Topográfico de humedad de la zona de estudio................................ 81

Figura 3.23: Elevación media por subparcelas............................................................. 81

Figura 3.24: Índice topográfico de humedad por subparcela ........................................ 82

Figura 3.25: Captura de pantalla de los feature-class de la base de datos geográfica ...... 83

Figura 3.26: Análisis de punto caliente en la parcela de 50-ha..................................... 85

Figura 3.27: Autocorrelación espacial incremental en la parcela de estudio. ................... 86

Figura 3.28: Histograma de frecuencias de la AGB en campo. ......................................... 90

Figura 3.29: Histograma de frecuencias del área basal. ........................................... 91

Figura 3.30: Histograma de frecuencias de la densidad de madera. ................................ 91

Figura 3.31: Matriz de diagramas de dispersión de las variables candidatas del modelo general. 93

Figura3.32: Malla regular de sub-parcelas de 1-ha usadas para la calibración y validación del modelo general de estimación de ACD usando LiDAR ................................................. 94

Figura 3.33: Análisis gráfico de los residuos. ................................................................. 96

Figura 4.1: Validación del modelo general de ACD ................................................... 101

Figura 4.2: Validación del modelo general de ACD por posiciones topográficas en: (a) valle; (b) ladera; (c) cima incluyendo el remanente de bosque secundario; (d) cima sin 
bosque secundario.

102

Figura 4.3: Relación entre el área basal estimada en campo y la altura media al tope del dosel (TCH) obtenida con LiDAR en la zona de estudio......

105

Figura 4.4: Mapa de altura media al tope del dosel (TCH) para la zona de estudio........ 107

Figura 4.5: Mapa de las reservas de carbono sobre el suelo en la zona de estudio. 108 


\section{LISTADO DE TABLAS}

Tabla 3.1: Factores diagnósticos del ecosistema BsTa02........................................... 54

Tabla 3.2: Factores diagnósticos del ecosistema BsTa09.............................................. 55

Tabla 3.3: Parámetros de recepción y posicionamiento de los 4 vértices geodésicos....... 65

Tabla 3.4: Coordenadas UTM ZONA 18 Sur de las bases observadas. ........................... 65

Tabla 3.5: Coordenadas UTM ZONA 18 Sur de las esquinas de la parcela de estudio.... 66

Tabla 3.6: Configuración del vuelo y del equipo LiDAR para la zona de estudio. ........... 69

Tabla 3.7: Resumen de las variables del modelo por posiciones topográficas. ................. 82

Tabla 3.8: Resumen de la autocorrelación espacial incremental en la parcela................ 87

Tabla 3.9: Coeficiente de variación de la densidad de AGB, área basal y densidad de madera en la parcela de estudio.................................................................................... 89

Tabla 3.10: Matriz de correlaciones de Pearson de las variables.................................. 92

Tabla 3.11: Tabla de probabilidad asociada de las variables del modelo........................ 92

Tabla 3.12: Resumen de las estadísticas del ajuste de regresión lineal múltiple.............. 95 


\section{LISTADO DE ACRÓNIMOS Y SIGLAS}

AGB: Biomasa sobre el suelo (Aboveground biomass).

ACD: Densidad de carbono sobre el suelo (Aboveground Carbon Density)

ALS: LiDAR aerotransportado (Airborne laser scanning).

ASPRS: American Society of Photogrammetry and Remote Sensing.

COP21: Vigesimoprimera Conferencia de las partes de la Convención Marco de las Naciones Unidas sobre el Cambio Climático.

CHM: Modelo de altura de vegetación (Canopy Height Model).

TWI: Índice de humedad por topografía (Topographic Wetness Index).

DAP: Diámetro a la altura de pecho.

DGPS: Sistema de Posicionamiento Global Diferencial (Differential Global Positioning system).

GEI: Gas de efecto invernadero.

IGM: Instituto Geográfico Militar de Ecuador.

INS: Sistema de Navegación inercial (Inertial navigation system).

LiDAR: Light Detection and Ranging.

MAE: Ministerio del Ambiente del Ecuador.

MCH: Altura media del perfil de dosel (Mean Canopy Height).

MDE: Modelo Digital de Elevación.

MDS: Modelo Digital de Superficie.

MDT: Modelo Digital del Terreno.

nMDS: Modelo Digital de Superficie Normalizado.

OBIA: Análisis de imágenes basada en Objetos (Object Based Image Analysis).

ONU: Organización de las Naciones Unidas.

REDD+: Reducción de Emisiones por Deforestación y Degradación de bosques.

RENAGE: Red Nacional GPS de Ecuador.

RMSE: Raíz cuadrada del error cuadrático medio (Root-Mean-SquareError).

SAR: Radar de Apertura Sintética (Synthetic Aperture Radar). 
SIRGAS: Sistema de Referencia Geocéntrico para las Américas.

TCH: Altura media al tope del dosel (Top of Canopy Height)

TIN: Red irregular de triángulos (Triangulated Irregular Network).

WD: Densidad o gravedad especifica de la madera (Wood Density). 


\section{$\boldsymbol{\theta 1}_{1}$}

\section{INTRODUCCIÓN}

“Biocombustibles, captura de carbono, vientos, fisión, fusión; somos imparciales, pero tiene que ser limpio, barato y escalable"

Bill Gates.

Cumbre COP21-Paris 



\section{INTRODUCCIÓN}

\subsection{Introducción}

Los sensores de teledetección, tanto espaciales como aerotransportados, han experimentado en los últimos años grandes avances en cuanto a sus resoluciones (espacial, radiométrica y espectral). La reciente capacidad del instrumental para combinar sensores activos y pasivos y su integración conjunta para su tratamiento en bases de datos espaciales, ha abierto la posibilidad de un amplio número de aplicaciones. El sensor LIDAR (Light Detection And Ranging) es uno de los sensores aerotransportados más avanzados en cuanto a su precisión altimétrica, convirtiéndose en el instrumento ideal para múltiples estudios entre ellos la caracterización de la estructura vertical de la vegetación. Este sensor ofrece la posibilidad de realizar un vuelo sincrónico con una cámara fotogramétrica digital, constituyendo ambos una magnífica fuente de información, con posibilidad de generar productos derivados (DMT, DMS, ortofotos en color e infrarrojo y clasificaciones estadísticas con los datos integrados de ambos sensores) de gran utilidad en la gestión de espacios forestales.

Los bosques tropicales son importantes reservorios de carbono y de biodiversidad, por lo que, caracterizar la distribución espacial de la biomasa sobre el suelo (AGB) y por consiguiente la densidad de carbono sobre el suelo (ACD o Aboveground Carbon Density) 
que contienen, aproximadamente el $48 \%$ del peso de la biomasa seca (Martin y Thomas, 2011) es un requisito previo para entender la dinámica espacial y temporal del ciclo de carbono terrestre. A fin de reducir las emisiones provenientes de la deforestación y la degradación forestal (REDD+), es necesario disponer de estimaciones precisas de ACD, y de cualquier cambio en las reservas de carbono debido a las actividades humanas, para contribuir a los esfuerzos de mitigación de cambio climático (Angelsen et al., 2008). Los bosques tropicales almacenan grandes cantidades de carbono, pero se desconoce su contribución exacta al ciclo global del carbono, y cómo ésta se distribuye en el espacio y el tiempo (Chave et al., 2005; Mitchard et al., 2014).

Las estimaciones de las reservas de ACD basadas en inventarios de campo y en enfoques de teledetección, han conducido a un progreso sustancial en el cartografiado de las reservas de carbono forestales a gran escala (Asner et al., 2010; Baccini \& Asner, 2013; Malhi et al., 2006; S. S. Saatchi et al., 2011). El método más común para la cuantificación de las reservas forestales de carbono a escala regional consiste en estratificar el área de interés, y luego asignar a cada estrato un valor medio estimado a partir de mediciones de campo. Este enfoque pasa por alto la extensa variación espacial en la densidad de carbono dentro de los estratos, incluyendo la variación relacionada con la degradación forestal y la regeneración, ambos componentes cruciales de los flujos de carbono forestal (Harris et al., 2012; Lewis et al., 2009). Por ello, estudios más recientes han pasado de enfoques basados en la clasificación, hacia enfoques que consideran la variación espacial continua de la estructura del bosque y la densidad de carbono, a menudo apoyadas en técnicas de teledetección espacial y aerotransportada (Asner et al., 2012a; Baccini \& Asner, 2013; Lefsky et al., 2002; Saatchi et al., 2011).

Las técnicas de teledetección activa como los sistemas LiDAR (Light Detection and Ranging), y los equipos Radar de Apertura Sintética (SAR - Synthetic Aperture Radar), constituyen en la actualidad las mejores herramientas para el cartografiado del carbono forestal a escalas espaciales grandes. Los enfoques más empleados para la predicción de ACD con LiDAR, se basan en modelos de regresión que combinan métricas obtenidas con LiDAR, con las estimaciones de campo obtenidas en parcelas forestales concretas. Con ello, el modelo resultante se utiliza para evaluar el ACD en áreas geográficas más grandes. La precisión de las estimaciones de carbono forestal resultantes depende fundamentalmente de la calidad de las mediciones de campo y de los procedimientos de calibración (Réjou- 
Méchain et al., 2014); es decir, de la capacidad de los datos LiDAR para predecir la ACD de un conjunto de parcelas de campo según lo evaluado por el modelo de regresión.

En los últimos años, algunos estudios para estimar ACD con LiDAR en bosques tropicales, se han realizado usando un enfoque de escalamiento alométrico de parcelas agregadas (Asner et al., 2012b, 2013; Taylor et al., 2015). La metodología se basa en un modelo general simplificado que demuestra que la biomasa seca del árbol, y su contenido de carbono, aproximadamente el 48\% del peso de la biomasa seca (Martin y Thomas, 2011), puede estimarse a partir de mediciones de la altura media al tope del dosel (TCH - Top of canopy height) obtenidas de LiDAR, mediciones espacialmente explicitas de área basal, e información de la densidad o gravedad específica de la madera. Este nuevo enfoque ha proporcionado estimaciones comparables en su capacidad de predicción, con los modelos calculados localmente (Asner \& Mascaro, 2014), y tiene el potencial de reducir el tiempo requerido para calibrar los datos LiDAR. Sin embargo, es necesario realizar pruebas adicionales para verificar su aplicabilidad en otras regiones tropicales.

La distribución de las reservas de carbono forestal es muy irregular debido a los efectos de complejos e interactivos procesos abióticos, como por ejemplo, el clima, suelos, geología, topografía, hidrología (Asner et al., 2009), y debido a procesos bióticos, como por ejemplo, la composición de las comunidades, y las actividades humanas (Baldeck et al., 2013; Malhi et al., 2006). En este sentido, una de las principales variables ambientales que determinan la cantidad de carbono sobre el suelo en bosques tropicales es la topografía (de Castilho et al., 2006; Taylor et al., 2015). Este hecho también se puso de manifiesto en el estudio realizado por Réjou-Méchain et al. (2014) sobre la estructura espacial de la biomasa forestal en 30 parcelas grandes permanentes (8-50 ha) distribuidas globalmente, destacando la importancia de la topografía, y sugiriendo que el muestreo de campo debe ser estratificado por posiciones topográficas, como por ejemplo, en cimas, valles y laderas.

Por otro lado, el tamaño de las parcelas de campo es un parámetro de diseño importante tanto en el inventario forestal tradicional, como en el inventario utilizando LiDAR. Para estudios de dinámica forestal en bosques tropicales es necesario contar con parcelas grandes, ya que permiten incluir poblaciones importantes de la mayoría de especies presentes en el entorno (Condit et al., 2000). Zolkos et al. (2013) realizaron una revisión detallada de varios estudios publicados sobre la estimación de AGB utilizando LiDAR, y 
concluyeron que el nivel de incertidumbre entre las estimaciones de AGB con LiDAR y las estimaciones sobre el terreno es de alrededor del 10\% cuando el tamaño de la parcela es de aproximadamente 1 ha. Estos resultados confirman que las parcelas de aproximadamente 1 ha en tamaño, generalmente dan resultados suficientemente precisos en la estimación de la biomasa forestal (Meyer et al., 2013). En un estudio reciente en tierras bajas de la Amazonía Occidental, Marvin et al. (2014), encontraron sesgos medios sustanciales en la estructura del dosel del bosque y la biomasa aérea (9-98\%); y que para estimar la ACD de manera confiable (es decir, una probabilidad de 0,9$)$ para una escala media de paisaje $\left(10^{2}-10^{4} \mathrm{ha}\right)$, sería necesario un tamaño de muestra promedio de 44 parcelas de 1 ha. El estudio sugiere que, cuando se realicen calibraciones con LiDAR, se haga una cuidadosa cuantificación de la variabilidad espacial de la biomasa y de la estructura vertical de la vegetación.

En el Ecuador, el tema de cambio climático es tratado concertadamente dentro de la política nacional, donde la evaluación de los bosques es un tema prioritario, por lo que, es un tema relevante la caracterización y valoración de sus servicios ecosistémicos, servicios tales como el carbono almacenado (MAE, 2013). Según MAE (2013), los bosques de tierras bajas de la Amazonía occidental son escenarios de gran importancia para la conservación y provisión de servicios ecosistémicos, tales como el almacenamiento de carbono y la conservación de la biodiversidad. Estos ecosistemas contienen una alta concentración de especies, convirtiéndola en una de las regiones de mayor biodiversidad en el mundo (Bass et al., 2010). De ahí que, disponer de información sobre la geografía del almacenamiento del carbono terrestre en esta región, es fundamental para comprender numerosos patrones y procesos ecológicos: desde distribuciones de hábitat y biodiversidad, a ciclos de carbono global e interacciones clima-biosfera.

En función de lo anteriormente descrito, las preguntas de investigación quedan expresadas en los términos que se indican a continuación:

\subsection{Preguntas de investigación}

En el contexto indicado, las preguntas de investigación que sustentan ésta tesis son:

1. ¿Es factible aplicar un modelo general de estimación de densidad de carbono sobre el suelo (que ha sido calibrado en bosques de otras regiones tropicales) en un 
bosque tropical con alta diversidad forestal de la Amazonía occidental, con datos obtenidos con tecnología LiDAR aerotransportada?

2. ¿Cómo influye la topografía del terreno en las estimaciones de la ACD obtenidas con tecnología LiDAR sobre un bosque tropical en la Amazonía occidental en Ecuador?

\subsection{Objetivos}

Para la resolución de las preguntas de investigación planteadas anteriormente, se definen los siguientes objetivos.

\subsubsection{General}

Desarrollar una metodología para calibrar y validar un modelo general de estimación de densidad de carbono sobre el suelo usando captura de datos con tecnología LiDAR aerotransportado, en un sector del Parque Nacional Yasuní en la Amazonía Occidental.

\subsubsection{Específicos}

Partiendo del objetivo general, se definen los siguientes objetivos específicos:

a) Realizar estimaciones de biomasa en campo, utilizando información proveniente del censo forestal de una parcela de 50 hectáreas.

b) Analizar la variabilidad local de la biomasa para identificar patrones de distribución espacial, utilizando modelado geo-estadístico.

c) Plantear un esquema de muestreo estratificado que considere la topografía del terreno, en base a variables geomorfométricas derivadas de los datos LiDAR.

d) Calibrar y validar un modelo general de estimación de ACD usando información LiDAR, y evaluar la incidencia de la topografía en la precisión de las estimaciones.

e) Elaborar el mapa de reservas de carbono sobre el suelo de la zona seleccionada a 
partir del modelo calibrado; y evaluar la incertidumbre asociada.

\subsection{Hipótesis de la investigación}

Las hipótesis de investigación planteadas en esta tesis son:

a) Se puede utilizar el modelo general de estimación de densidad de carbono sobre el suelo propuesto en la literatura, que ha sido utilizado en estudios similares en otros entornos tropicales.

b) Se puede mejorar la precisión de las estimaciones de densidad de carbono sobre el suelo si se utiliza un esquema de muestreo estratificado, que considere la topografía, utilizando variables geomorfométricas derivadas del Modelo Digital de superficie obtenido con datos LiDAR.

\subsection{Estructura de la tesis y metodología utilizada}

La tesis consta de 5 capítulos y 8 anexos. El presente capítulo introductorio, plantea el origen del problema de investigación y los objetivos, hipótesis, y metodología propuestos para su resolución.

En el capítulo 2 se realiza una revisión del estado del arte sobre el uso de sensores LiDAR aerotransportados en los inventarios forestales, poniendo énfasis en la estimación de biomasa sobre el suelo en bosques tropicales. Su contenido procura describir los antecedentes, los principios teóricos básicos y necesarios, que fundamenten la propuesta metodológica para el cartografiado de densidad de carbono sobre el suelo en el bosque tropical amazónico del Ecuador, que será utilizada en el capítulo 3.

En el capítulo 3, por medio de un estudio de caso en una zona de bosque tropical en Ecuador con alta diversidad florística, se calibra y valida el modelo general de estimación de densidad de carbono sobre el suelo (ACD) usando LiDAR aerotransportado. Para ello, inicialmente, se realiza la descripción detallada del área de estudio, y de los datos de campo disponibles para la calibración y validación del modelo general. A continuación, se explica el procedimiento utilizado para la captura y procesamiento de datos LiDAR, y se plantea 
una propuesta de diseño de muestreo estratificado usando productos derivados de LiDAR. Finalmente, se analiza la distribución de ACD en el área de estudio usando técnicas de modelado geoestadistico; y se realiza el ajuste del modelo general.

En el capítulo 4 se analizan los resultados obtenidos con el desarrollo metodológico descrito en el capítulo 3. Se realiza la validación del modelo general con el que se genera el mapa de reservas de ACD de la zona de estudio, y se efectúa el análisis de la incertidumbre del mapa obtenido.

En el capítulo 5 se presentan las conclusiones de la investigación, así como las aportaciones, líneas de investigación futuras identificada en el estudio y cuál ha sido la difusión de la investigación hasta el momento.

Finalmente se presentan las referencias bibliográficas utilizadas y los anexos con la información que complementa los procedimientos desarrollados en los distintos capítulos.

A continuación, en la figura 1.1, se presenta el esquema general de los procedimientos utilizados en la investigación para determinar el mapa de carbono en la zona de estudio: 


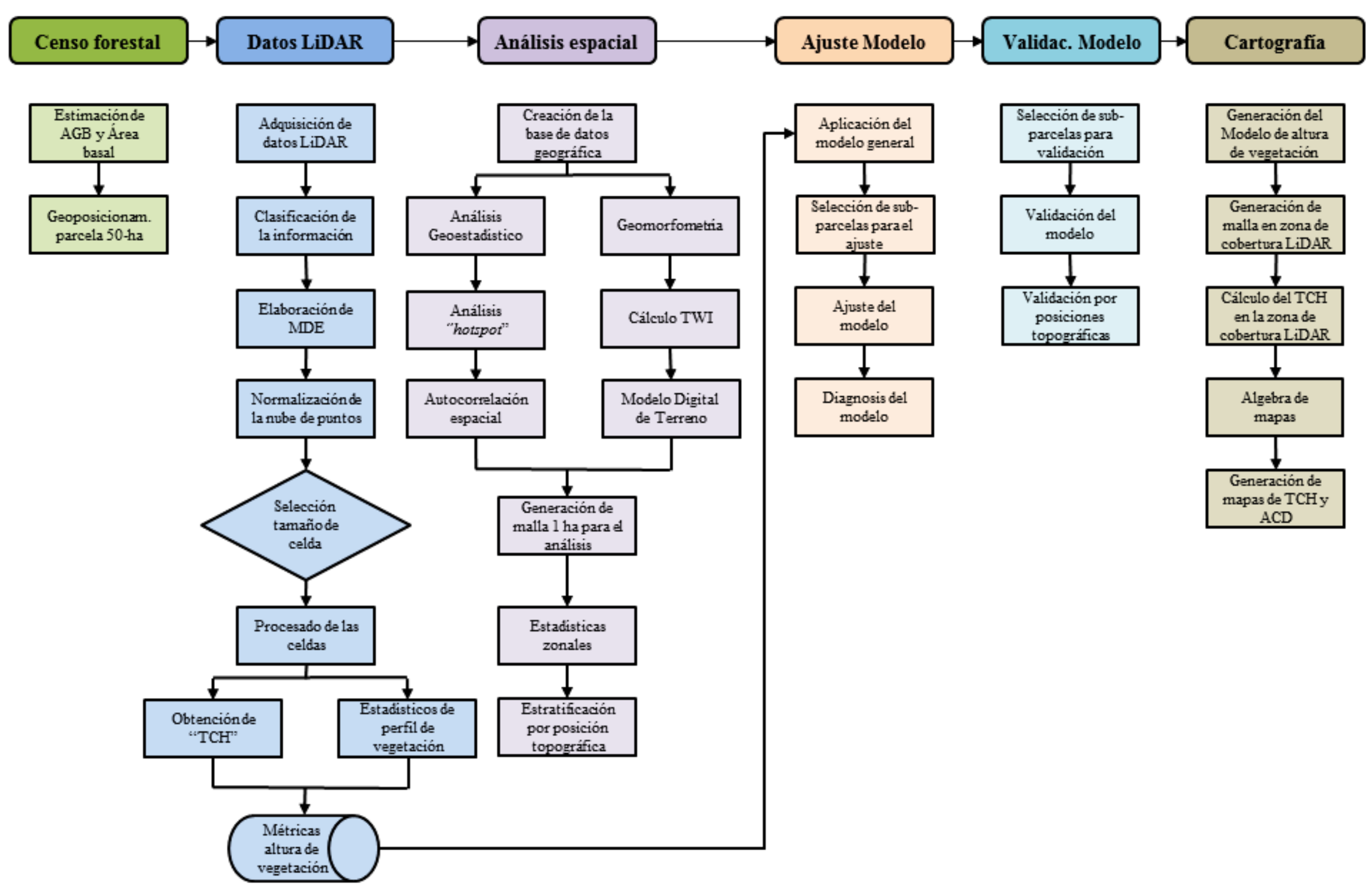

Figura 1.1: Unidades metodológicas. 


\section{MARCO TEÓRICO}

"De vez en cuando vale la pena salirse del camino, sumergirse en un bosque.

Encontrará cosas que nunca había visto".

Alexander Graham Bell 


\section{Resumen:}

En este capítulo se realiza la revisión del estado del arte sobre el uso de sensores LiDAR aerotransportados en los inventarios forestales, poniendo énfasis en la estimación de la biomasa sobre el suelo en bosques tropicales. Su contenido procura describir los antecedentes, los principios teóricos básicos y necesarios, que fundamentan la propuesta metodológica para el cartografiado de densidad de carbono sobre el suelo en una parte del bosque tropical amazónico del Ecuador, propuesta metodológica que será implementada en un estudio de caso en el Parque Nacional Yasuní y que se describirá en el siguiente capítulo. 


\section{MARCO TEÓRICO}

\subsection{Introducción}

Desde el año de 1992, fecha en la que un gran número de países firmaron el programa sobre diversidad biológica ambiental en la convención de Río de Janeiro, las políticas nacionales e internacionales de conservación de la naturaleza han promovido el control y la conservación de la biodiversidad; especialmente en relación con los bosques. Después de más de veinte años, en la vigesimoprimera Conferencia de las Partes de la Convención Marco de las Naciones Unidas sobre Cambio Climático (COP21) realizada en París a finales del año 2015, de los más de 180 países que presentaron contribuciones nacionales a Naciones Unidas para el nuevo acuerdo de cambio climático, casi un centenar se comprometieron a reducir emisiones de $\mathrm{CO}_{2}$ a la atmosfera mediante la preservación o restauración de sus masas forestales. En una declaración conjunta, Alemania, Noruega y Reino Unido, se comprometieron a reunir US\$5.000 millones entre el año 2015 y el 2020, si los países con más bosques miden, informan y verifican reducciones de emisiones procedentes de la conservación o restauración de sus bosques. Entre los estados que aseguraron su compromiso se encuentran la mayoría de países latinoamericanos que estaban 
presentes en la cumbre.

En este ámbito, la teledetección y la evaluación en campo son la principal fuente de datos para los inventarios forestales y para los estudios de biodiversidad (Köhl et al., 2006). Si bien, la teledetección proporciona datos espacialmente explícitos para grandes extensiones, la cantidad de atributos que pueden ser obtenidos de estas imágenes son más bien limitados, en consecuencia, la evaluación en campo continúa siendo un componente esencial para los inventarios forestales. Holmgren y Thuresson, (1998) realizaron una revisión detallada, desde una perspectiva crítica, de 25 años de investigación de teledetección por satélite en el contexto de la planificación forestal. Si bien el estudio se limita a regiones templadas y boreales, y en el espectro infrarrojo visible y cercano, concluyen que las imágenes de satélite rara vez contienen información suficiente para apoyar el proceso de toma de decisiones en silvicultura aplicada, y que éstas pueden ser útiles para aplicaciones forestales a nivel regional, pero a nivel local, pocas aplicaciones han concluido con éxito y con el nivel de confianza suficiente.

\subsection{Aplicaciones LiDAR en el ámbito forestal}

Los estudios forestales y de biodiversidad requieren de cartografía de alta precisión que permita entender las funciones y la dinámica de los bosques, especialmente en zonas de extrema fragilidad y alta heterogeneidad como es el caso de los bosques tropicales. Dentro de este campo de estudio, el potencial de LiDAR para identificar y representar el bosque está reconocido en la literatura publicada hace ya más de treinta años, cuando Arp y Tranarg (1982) utilizaron LiDAR para cartografiar bosques en América Central. En esas fechas también el Servicio Forestal Canadiense realizó estudios de perfiles con altímetro láser para caracterizar la altura y la cobertura del dosel arbóreo en masas forestales (Aldred \& Bonnor, 1985).

A principio de los años 1990, la NASA empezó a utilizar sistemas de escaneo de onda continua, de huella mediana y grande, para realizar mediciones verticales de la estructura de la vegetación en bosques (Dubayah \& Drake, 2000). Éstos sensores también ha sido utilizados para la caracterización vertical de la estructura forestal (Zimble et al., 2003), en la detección de cambios en la vegetación (Yu et al., 2004), en la estimación de parámetros forestales para la generación de modelos de propagación de incendios forestales (Erdody \& Moskal, 2010), en la determinación de modelos del terreno en áreas boscosas (Kraus \& 
Pfeifer, 1998), para la extracción de unidades de gestión forestal en bosques tropicales (Hou et al., 2011), para la cuantificación de sumideros de $\mathrm{CO}_{2}$ en zonas forestales mediterráneas (García et al., 2010), y para la caracterización de áreas forestales en regiones tropicales usando OBIA (Dupuy et al., 2013), entre otras aplicaciones.

Los bosques son reservas importantes de carbono y de biodiversidad, y la caracterización de la distribución espacial de su biomasa sobre el suelo (AGB) es un requisito previo para el entendimiento de la dinámica del ciclo de carbono en el tiempo. En la actualidad, se requieren estimaciones exactas de AGB y de los cambios en las existencias de carbono resultado de las actividades humanas. Esto permite reducir las emisiones derivadas de la deforestación y la degradación forestal (REDD+), y contribuir con los esfuerzos en curso para la mitigación del cambio climático (Angelsen et al., 2008). En este marco, los bosques tropicales desempeñan un papel vital en el ciclo global del carbono, pero la cantidad de carbono que contienen y su distribución siguen siendo inciertas (Chave et al., 2005).

Durante la última década, la estimación de las reservas de captación de carbono de los bosques tropicales ha evolucionado a partir de una actividad basada en gran medida en los inventarios de campo (Malhi et al., 2006), hacia un enfoque asistido por teledetección aérea y espacial (Asner et al., 2010).

\subsection{Conceptos Fundamentales}

\subsubsection{Que es LiDAR}

El término LiDAR es un acrónimo utilizado para nombrar a Light Detection And Ranging; es una tecnología de detección activa que usa un sensor para emitir un pulso o haz de luz intenso y dirigido, y que mide el tiempo que tarda dicho pulso en reflejarse en un objeto y ser detectado de nuevo por el sensor. Esta información de tiempo, se usa para calcular rangos o distancias a los objetos. El escaneado láser aerotransportado (ALS Airborme Laser Scanning) también conocido como altímetro láser o LiDAR, permite la medición de la forma/topografía de la superficie de la tierra y de las características referentes a la altura y la intensidad de reflejo de los materiales. El $A L S$ está formado por un sistema de georreferenciación directa, que permite determinar con precisión la posición y orientación de la plataforma aérea, y de un sistema de escaneo láser que emite los pulsos de 
luz mencionados. Este equipo trabaja en combinación con un sistema de Posicionamiento Global Diferencial (DGPS) y un sistema inercial de navegación (INS), (figura 2.1):

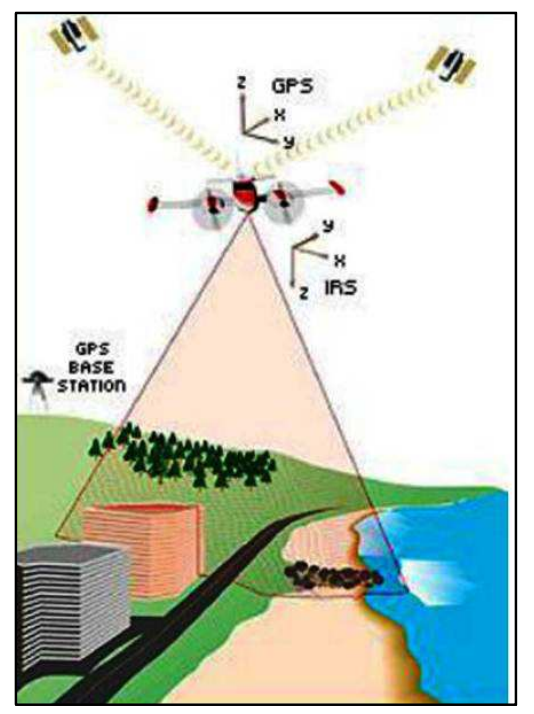

Figura 2.1: Componentes del sistema de datos LiDAR aerotransportado; Fuente: Aaron Smith, Forest Resources Remote Sensing and GIS Lab.

Los sensores LiDAR suelen emplear un láser en el infrarrojo próximo, normalmente entre los 1000 a 1500 nanómetros, y proporciona información tridimensional de la superficie escaneada. El resultado de este escaneo es una nube de puntos de elevación georreferenciados con alta precisión que contiene información de posición (tripleta de coordenadas de los puntos de incidencia del láser), y valores de intensidad de la señal láser, valores que pueden ser usados para elaborar representaciones tridimensionales de la superficie terrestre y de sus características. Las coordenadas tridimensionales $(\mathrm{x}, \mathrm{y}, \mathrm{z}$ o latitud, longitud y elevación) de los objetos reflejados se calculan a partir de: a) la diferencia de tiempo entre el haz emitido y reflejado, b) el ángulo de disparo del pulso y c) la posición absoluta del sensor sobre la superficie de la tierra.

En la actualidad, los sistemas LiDAR son mucho más avanzados en comparación a versiones anteriores de sistemas de escaneo láser, que eran capaces de grabar solo un pulso a tasas de repetición bajas. Los fabricantes han desarrollado sensores que pueden grabar simultáneamente múltiples ecos o multi-retorno para cada pulso de láser transmitido y la intensidad reflejada para cada eco (figura 2.2). En los últimos años, se han introducido sistemas que capturan la forma de onda completa, la digitalización completa de la forma de onda ofrece muchas opciones, ya que permite obtener muchos retornos múltiples con una separación corta (posiblemente impulsos a cada $15 \mathrm{~cm}$ de recorrido) con un solo disparo 
láser. El lado negativo de esta mejora es que, a más retornos recolectados, el almacenamiento de datos es mayor, y se requiere un procesamiento de datos más complejo.
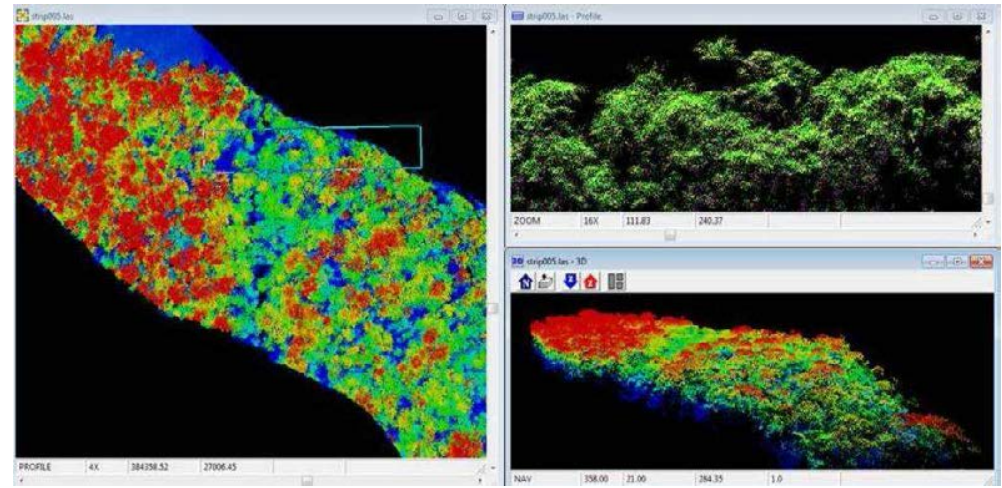

Figura 2.2: Nube de puntos LiDAR multi-retorno de un sector del bosque tropical en la Provincia de Sucumbíos-Ecuador; Fuente: Equipo LiDAR del IGM.

La capacidad multi-retorno es fundamental para entender las aplicaciones forestales del LiDAR, ya que permite describir con precisión la estructura de la vegetación capturando información tridimensional del suelo y de los diferentes estratos (figura 2.3).

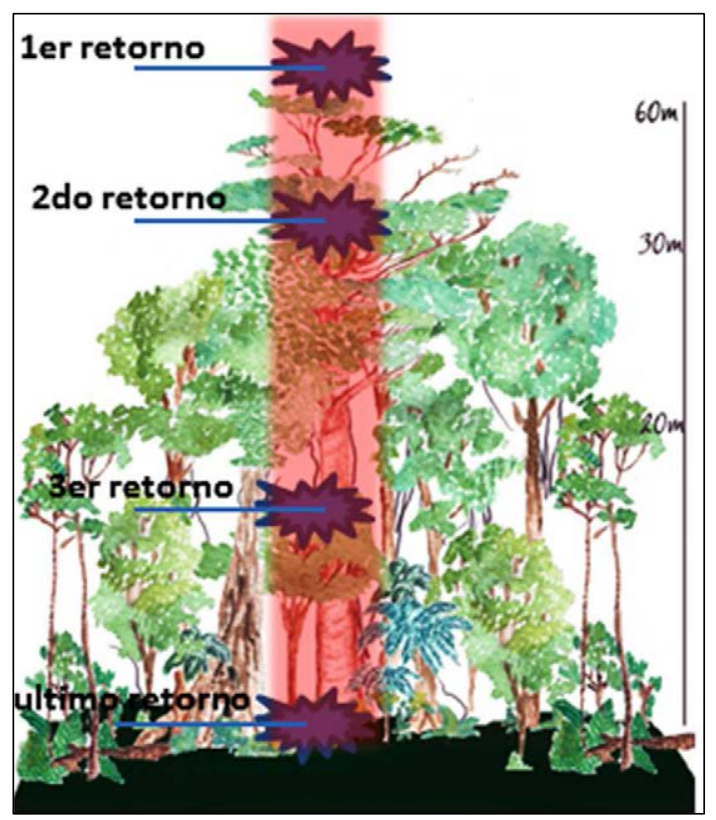

Figura 2.3: Esquema de múltiples retornos del pulso de luz láser en bosque tropical; Fuente: imagen adaptada de mapas.centrogeo.org.mx.

En superficies compactas sin vegetación se obtiene un único retorno (todo el haz de luz es reflejado), en cambio en superficies con vegetación el sensor es capaz de registrar varios retornos para un mismo pulso de luz láser ya que es reflejado parcialmente por la 
vegetación. En bosque tropical, la situación es más complicada, cuando el haz de luz láser de un sistema multi-retorno alcanza un árbol, se asume que el primer retorno proviene de cerca de la parte superior de la cubierta forestal; el último retorno, se asume que proviene del suelo. Los retornos intermedios, es decir el $2^{\circ}, 3^{\circ}, 4^{\circ}$ se espera que provengan de las ramas de los árboles, sotobosque entre la parte superior de la cubierta forestal y el suelo.

Sin embargo, a veces, la vegetación es tan densa que el rayo láser no alcanza el suelo. Normalmente, la regla de oro es: si una persona que camina a través del bosque no puede ver el cielo por encima del dosel, probablemente el sistema LiDAR no podrá "ver" el suelo bajo el dosel. La figura 2.4 muestra el dosel forestal en la zona de estudio:

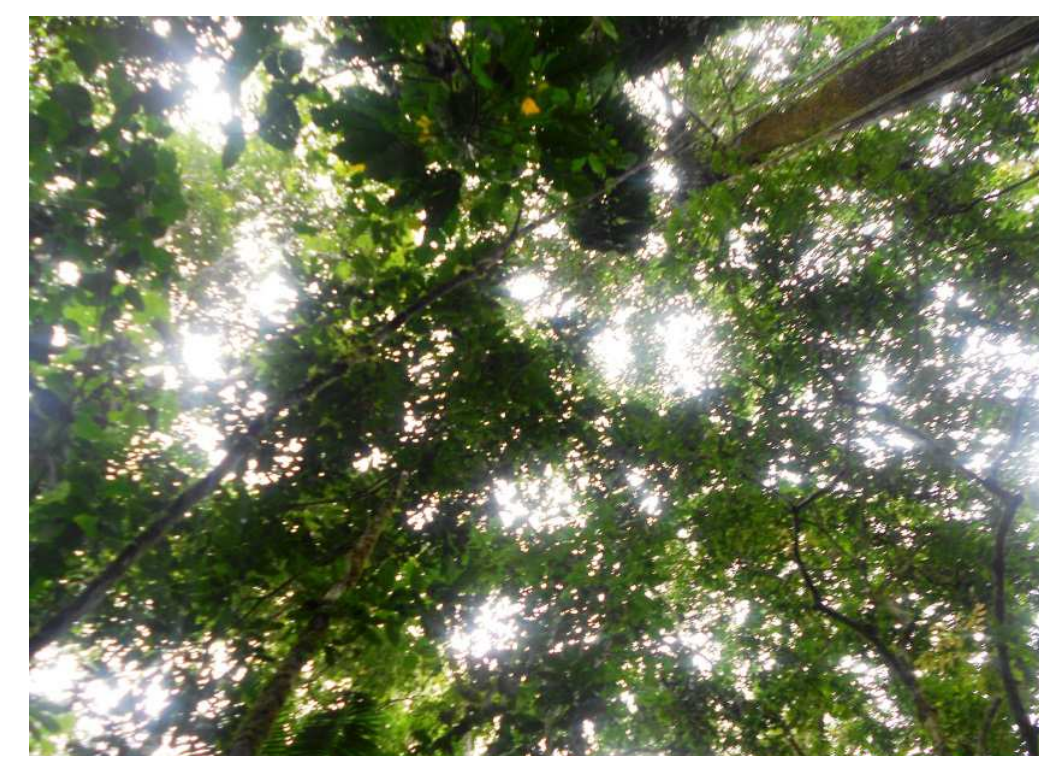

Figura 2.4: Fotografía del dosel forestal en la zona de estudio.

\subsubsection{Estructura tridimensional de la vegetación forestal}

El empleo de sistemas LiDAR, está transformando la forma en que se llevan a cabo en la actualidad los estudios forestales. Con este tipo de tecnología, se pasa de una concepción de disponibilidad de información bidimensional en pixeles, a una concepción tridimensional con un formato denominado Voxel (volumetric pixels-fig.2.5). El uso del voxel y la visualización del volumen, demuestra un excelente potencial en la representación de los datos LIDAR de retorno múltiple, y permite conocer el porcentaje de puntos LiDAR dispuestos en cada voxel y por tanto sistematizar su análisis (Stoker, 2009). Una de las aplicaciones de la interpretación de los datos LiDAR para el inventario forestal, es la estimación directa de parámetros forestales o silvícolas a las que se añaden los demás 
parámetros deducidos o que pueden ser modelados.

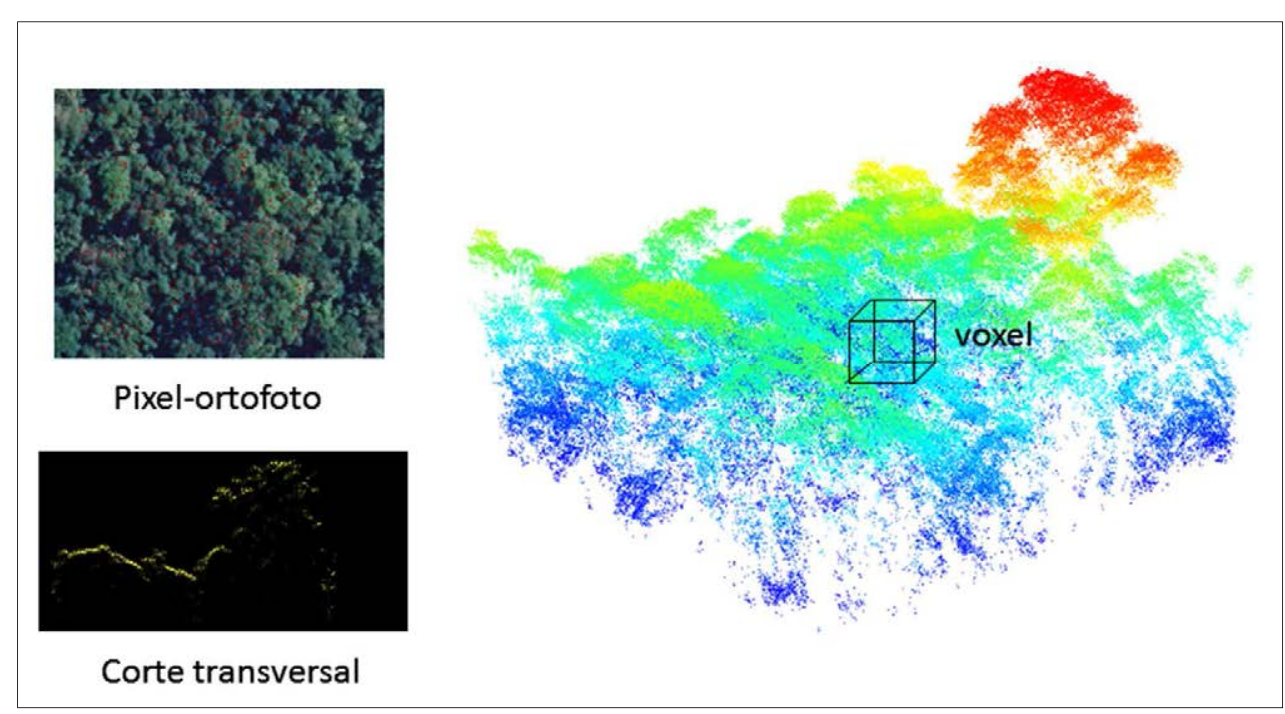

Figura 2.5: Concepto de voxel en el análisis de la estructura vertical del bosque; imagen adaptada de Dielmo.com.

\subsubsection{Métricas o estadísticos LiDAR}

Las métricas de LiDAR son los estadísticos descriptivos de los datos láser. Teóricamente, puede haber un número infinito de posibles métricas derivables de un conjunto de puntos LIDAR. Los indicadores de forma de la onda también dependen de las características de los datos LIDAR, tales como el número de retornos registrados por pulso y si la información auxiliar tales como la intensidad y el ángulo de escaneo, están disponibles. En los estudios forestales, las medidas LiDAR más utilizadas incluyen entre otros estadísticos las alturas medias, los percentiles de altura.

\subsection{Principales variables y parámetros de estructura forestal inferidos de datos LiDAR}

Las métricas obtenidas a partir de LiDAR están correlacionadas con variables biofísicas de árbol debido a la naturaleza volumétrica de las mediciones en 3 dimensiones (Drake et al., 2003; Stocker, 2009). El uso de LiDAR, permite una elevada precisión en las mediciones y buena predicción de las variables dasométricas (Dubayah \& Drake, 2000; Means \& Acker, 2000; Lim et al., 2003; Reutebuch et al., 2005). A continuación, se describen las variables más utilizadas en el ámbito forestal. 


\subsubsection{Altura de dosel arbóreo}

La altura del dosel del bosque es la característica biofísica más común y es de gran importancia para los estudios forestales, constituye además el elemento clave en la estimación de biomasa en los bosques (Feldpausch et al., 2012; Hunter et al., 2013). La medición de la altura de un árbol individual (figura 2.6), se define como la distancia vertical desde la base del árbol hasta su parte terminal superior. La medición de la altura de los árboles usando LiDAR se estima a partir de la resta entre el retorno de elevación más alto, y el retorno de elevación más bajo y más cercano al árbol, y que pertenece al modelo digital del terreno (MDT). De este modo los datos LiDAR han demostrado ser eficientes en la estimación de la altura de los árboles (Naesset \& Næsset, 1997).

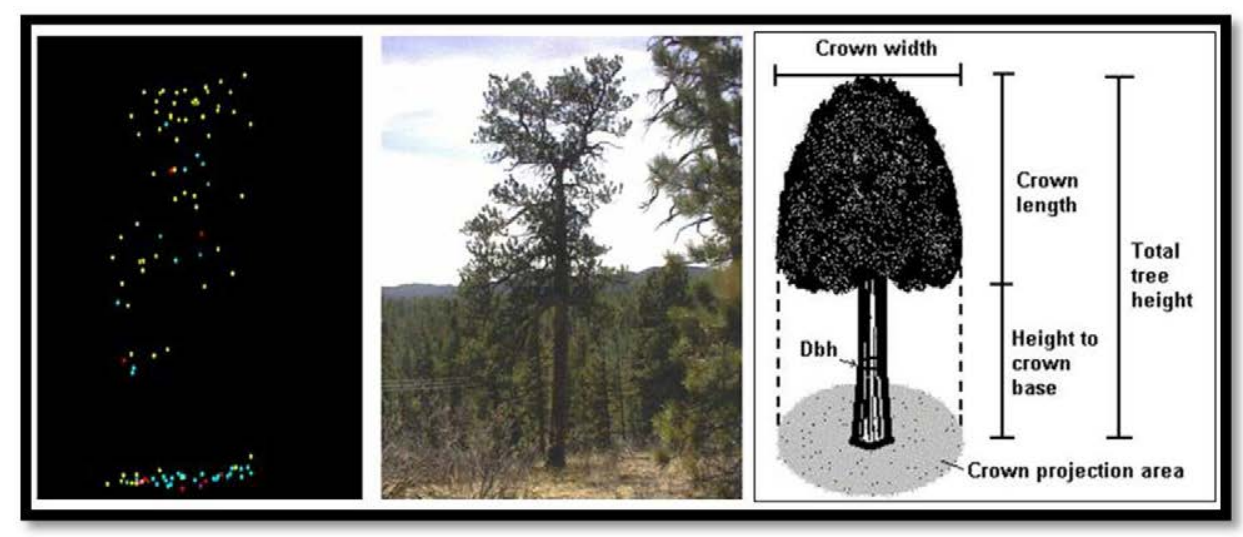

Figura 2.6: Distribución de retornos LiDAR de un árbol individual y el suelo y sus mediciones típicas; adaptado de Stoker y U.S Forest Service Remote Sensing Application Center.

\subsubsection{Longitud de copa (Crown length)}

Un atributo importante en el árbol es la longitud de la copa. Para calcular este parámetro, se resta el valor más alto de elevación registrado del valor más bajo registrado en su copa. En un estudio con LiDAR para estimar la altura de los árboles y las propiedades de su copa en un bosque boreal, Næsset y Økland (2002), plantearon la hipótesis que la longitud de copa podría ser estimada directamente de datos láser, especialmente cuando las copas de los árboles adyacentes se encuentran separados unos de otros, e inclusive en masas forestales densas donde las copas interfieren entre sí. En bosques tropicales, el tamaño de la copa de los árboles, podría influenciar en las estimaciones de biomasa cuando se utiliza LiDAR (Goodman et al., 2014). 


\subsubsection{Diámetro a la altura de pecho y área basimétrica}

La variable más común y más importante utilizada en la medición de árboles y masas forestales es el diámetro del árbol, medición a partir de la que se calcula el área de la sección transversal del tronco del árbol para poder estimar el volumen, o su biomasa. La convención universal es medir el diámetro del árbol con corteza a menos que se especifique lo contrario, a una altura fija desde el nivel del suelo. Esta altura estándar es la altura del pecho y puede variar ligeramente entre algunos países. Las mediciones del diámetro a la altura de pecho (DAP) son utilizadas para calcular estimaciones de volumen de madera, área basal y biomasa sobre el suelo de árboles individuales y bosques enteros.

El Área Basal o Área Basimétrica, es la relación existente entre la suma de las superficies de las secciones normales de los árboles de una determinada masa forestal, expresadas en $\mathrm{m}^{2}$, y la superficie del terreno que ocupan, expresada en hectáreas. El área basal total constituye una medida de la densidad del bosque que refleja la cantidad de árboles y su tamaño. Lefsky et al. (1999) desarrollaron ecuaciones para estimar el área basimétrica y la biomasa relacionando índices de altura obtenidos con LiDAR utilizando métodos de regresión lineal.

\subsubsection{Fracción de cabida cubierta (fractional canopy cover)}

La fracción de cabida cubierta (FCC) se refiere a la proporción del suelo del bosque cubierto por la proyección vertical de las copas de los árboles (Jennings et al., 1999). La figura 2.7 ilustra el concepto de fracción de cabida cubierta usando el primer retorno LiDAR. De los 21 pulsos LIDAR que se adentran en el dosel, los 16 primeros retornos se registran por encima del umbral de 3 metros o altura de quiebre (break height). El valor de la FCC según este autor queda definido por la ecuación 2.1:

$$
76 \%=\frac{16}{21} * 100
$$

Esta relación se puede expresar de la siguiente manera (ecuación 2.2):

$$
\text { FCC }(\%)=\frac{\text { número de primeros retornos }>\text { a la altura de quiebre }}{\text { número total de retornos }} * 100
$$




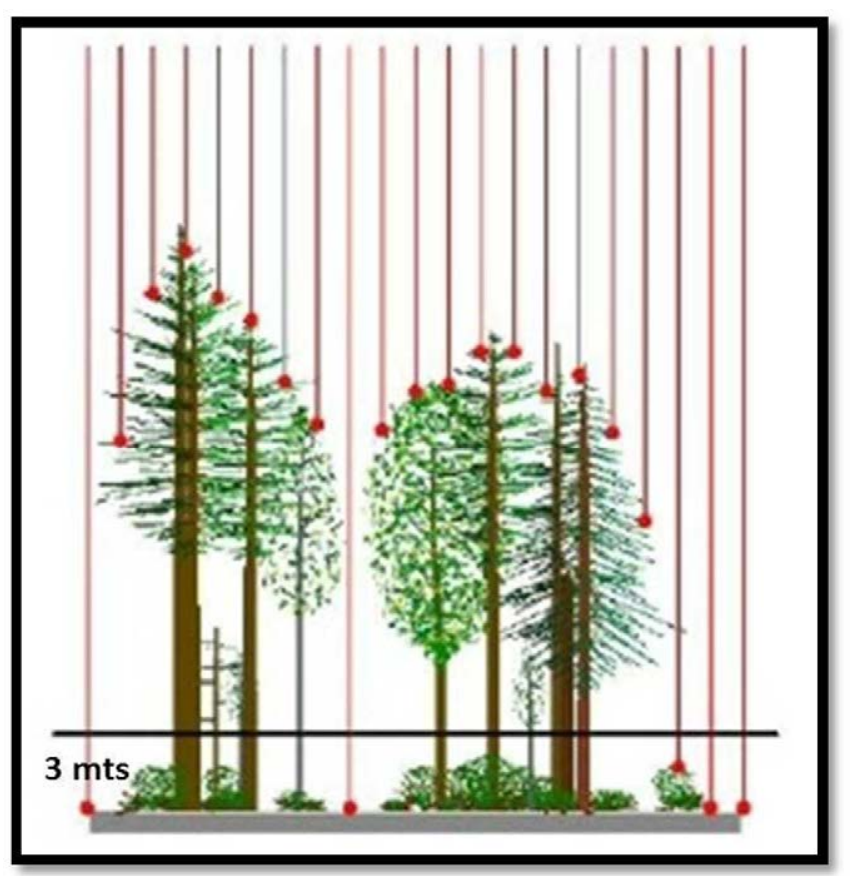

Figura 2.7: Esquema del proceso de cálculo de la cobertura de copas; Fuente: McGaughey, R.J., (2013).

Hay dos variables importantes a considerar cuando se realiza el cálculo de la FCC con LiDAR: a) la altura de quiebre, y b) el tamaño de la celda. Para producir estimaciones de FCC significativas, el tamaño de la celda debe ser más grande que la copa del árbol. Para la mayoría de los tipos de bosque, tamaños de celda de 15 metros o más grandes se obtienen resultados validos (McGaughey, 2013).

\subsubsection{Biomasa forestal}

La biomasa de un árbol se refiere al peso o masa del tejido vivo de su planta y se expresa generalmente en unidades de toneladas métricas (Walker et al., 2011). La biomasa viva puede ser separada en: a) biomasa sobre el suelo (hojas, ramas y tallo) o también llamada biomasa aérea, y b) biomasa bajo el suelo (raíces). La biomasa sobre el suelo de cada árbol, es el peso del tejido vivo de la planta después de que toda el agua haya sido removida, es decir, después de que las hojas, ramas y tallos se hayan secado por completo, a menudo utilizando un horno especial de laboratorio. En general, el agua representa aproximadamente el 50\% o la mitad del peso (o biomasa húmeda) de un árbol vivo (Figura 2.8). 


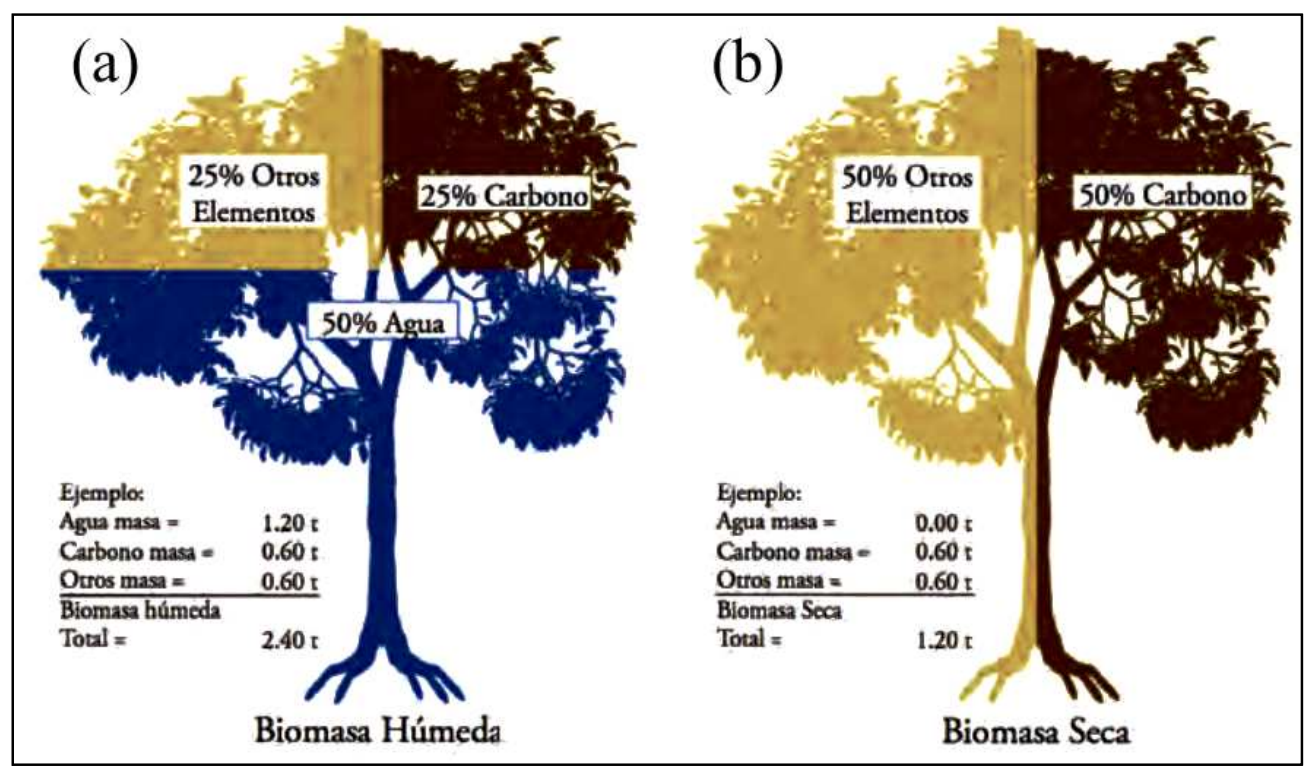

Figura 2.8: La proporción relativa de carbono (C), Agua (H2O), y otros elementos (e.g. N, P, K, Ca, Mg, etc.) contenida en la biomasa (a) húmeda y (b) seca de los árboles; Fuente: Walker et al. (2011).

Debido a las propiedades biofísicas de los árboles, el diámetro del tronco generalmente aumenta a medida que los árboles se vuelven más altos, aumentando aún más el volumen de madera y en consecuencia de biomasa. Las mediciones LiDAR pueden utilizar este resultado biológico para modelar la biomasa a partir de la altura, y estas estimaciones son esenciales para comprender mejor los ciclos de carbono sobre biomas donde los bosques sirven como una fuente primaria de biomasa y carbono terrestre.

\subsubsection{Carbono forestal}

Los bosques proporcionan una gran variedad de beneficios, que incluyen desde la purificación del aire, protección de las cuencas hidrográficas y la conservación de la biodiversidad, hasta constituirse en fuentes de alimento, fibra y medicina (Walker et al., 2011). Los bosques también desempeñan un papel importante en el mantenimiento de la estabilidad del clima global. Debemos tomar en cuenta que los árboles y otras plantas forestales remueven, a medida que crecen, grandes cantidades de dióxido de carbono $\left(\mathrm{CO}_{2}\right)$, un gas de efecto invernadero (GEI), de la atmósfera, almacenando el carbono en la biomasa de sus hojas, ramas, tallos y raíces. De este modo los bosques tienen gran capacidad para la captación y almacenamiento de carbono, además de reducir estas emisiones de GEI provenientes de los combustibles fósiles, por lo que, el uso sostenible de los bosques es una de las maneras más efectivas para eliminar el carbono de la atmósfera. 
Para una adecuada gestión y planificación sostenible de los bosques, se necesita realizar estudios detallados sobre su composición y su estructura florística, así como sobre las cantidades de carbono que contienen. Estos estudios se realizan mediante inventarios forestales a nivel de árbol y a nivel de masas forestales (Leppänen et al., 2008). Estos inventarios permiten entender las funciones y la dinámica de los bosques, su biodiversidad y el contenido de carbono en la vegetación, especialmente en zonas de extrema fragilidad y alta heterogeneidad como es el caso de los bosques tropicales. Las técnicas en campo para cuantificar la biomasa y el carbono en los bosques tropicales, presentan limitaciones debido principalmente a las metodologías y procedimientos utilizados para realizar las estimaciones (Marvin et al., 2014). Esto ha generado incertidumbres sobre la función que desempeñan estos ecosistemas en el ciclo y balance global del carbono, dificultando la implementación eficiente de proyectos de REDD+.

La Reducción de Emisiones de gases de efecto invernadero causadas por la Deforestación y Degradación de los bosques, la conservación y el incremento de las capturas de $\mathrm{CO}_{2}$, también conocida como REDD+, es un mecanismo de mitigación del cambio climático desarrollado bajo la Convención Marco de las Naciones Unidas sobre Cambio Climático (CMNUCC). Este programa busca reconocer y proveer incentivos a los países en vías de desarrollo para que protejan sus recursos forestales, mejoren su gestión y hagan uso de los mismos de manera sostenible, con el fin de contribuir a la lucha global contra el cambio climático y sus efectos.

Desde el punto de vista de REDD+, el aumento de la incertidumbre en las estimaciones de carbono forestal, conlleva una reducción del valor monetario del carbono forestal (Grassi et al., 2008). Otro estudio (Plugge et al., 2013) menciona que precisiones por debajo del 95\%, podrían excluir (o reducir en gran medida) a la mayoría de naciones, de los beneficios monetarios por conservar los bosques. Dado que la mayoría de los puntos calientes de biodiversidad del mundo se encuentran dentro de las regiones de bosques tropicales (Myers et al., 2000), los beneficios monetarios podrían no sólo asegurar el almacenamiento de carbono, sino también, la reducción de la reconversión de regiones de alta biodiversidad y, potencialmente, ayudar al desarrollo económico y social de un país (CCBA, 2013).

La tecnología LiDAR ha demostrado ser útil para estimar patrones de densidad de carbono, y reducir las incertidumbres de las estimaciones en todos los entornos forestales; 
sin embargo, la precisión y la generalidad de las calibraciones entre las predicciones de densidad de carbono sobre el suelo asistidas por LiDAR, y las estimaciones basadas en técnicas de inventario de campo, se deben incrementar con el fin de hacer más accesible el cartografiado de carbono de los bosques tropicales (Asner \& Mascaro, 2014).

\subsection{Normalización de la nube de puntos LiDAR}

Un paso fundamental en el procesado de la información LiDAR para aplicaciones forestales es la normalización de la nube de puntos. Generalmente, un modelo Digital de Superficie Normalizado (nMDS), o modelo de altura de vegetación (CHM - canopy height model), se calcula restando el Modelo Digital de Superficie (MDS) del Modelo Digital del Terreno (MDT) (Hyyppä et al., 2004). En otras palabras, a cada retorno de vegetación se le extrae la altura del terreno, para así obtener la altura de cada retorno de vegetación sobre el suelo (ecuación 2.3):

$$
\text { nMDS }=\text { MDS }- \text { MDT }
$$

La estimación de la altura de la vegetación del bosque a partir de datos LIDAR depende de una representación exacta de la superficie del suelo. En este sentido, LiDAR es capaz de caracterizar de forma eficaz tanto terreno como la estructura de la vegetación. Sin embargo, cuando se producen errores en el MDT, estos errores se propagan y afectan la precisión de las métricas de vegetación y los modelos digitales de copas (Tinkham et al., 2012). En zonas cubiertas por vegetación densa, como el caso de los bosques tropicales, los errores en el MDT tienden a aumentar, ya que llega al suelo menos energía, lo que provoca que se cuente con un menor número de puntos de tierra para la interpolación del MDT (Clark et al., 2004).

\subsubsection{Clasificación o filtrado de puntos LiDAR}

Para obtener el MDT es necesario clasificar la nube de puntos LiDAR según pertenezcan al suelo, o a objetos por encima de la superficie topográfica. A este proceso se le denomina filtrado de puntos, y se realiza utilizando diferentes tipos de algoritmos (Baltsavias, 1999).

Los algoritmos de filtrado se pueden agrupar en las siguientes categorías (Sithole \& Vosselman, 2004): a) algoritmos basados en el cálculo del TIN, b) contornos activos, c) interpolación, d) morfológicos y e) basados en la pendiente. Un conjunto de estudios 
esperimentales realizados por Sithole y Vosselman (2004) en ocho áreas geográficas, demostraron que el método basado en TIN presenta el mejor desempeño en términos de precisión promedio global.

Una revisión detallada sobre temas críticos de esta problemática analiza cuestiones como las siguientes: el desarrollo y aplicación de algoritmos de filtrado de suelo, los procedimientos de filtrado para diferentes tipos de entidades, los criterios para la selección del sitio de estudio, la evaluación de la precisión y el algoritmo de clasificación es presentada por Meng et al. (2010) y Xiaoye (2008). Un estudio comparativo entre algoritmos demuestra que el dosel de bosque es uno de los mayores factores que afectan la precisión de los MDT en áreas forestales ya que evita que gran parte de los pulsos láser alcancen el suelo subyacente (Hyyppä et al., 2004).

En un estudio realizado por Leitold et al. (2015) en bosque tropical Amazónico montano y sub-montano en Brasil (0-1200 msnm), se demuestra que la capacidad para generar un MDT de alta precisión $(0.19 \pm 0.97 . \mathrm{m})$ se puede atribuir en parte, a la densidad alta de puntos LiDAR (20 retornos $\mathrm{m}^{-2}$ en promedio), a pesar de las grandes diferencias de elevación y dosel cerrado de la cubierta forestal tropical.

\subsubsection{Modelo Digital del Terreno (MDT)}

En aplicaciones forestales es extremadamente importante contar con un MDT fiable, ya que constituye la base para el cálculo de la altura de los árboles. La variabilidad en la exactitud del MDT introduce errores en los cálculos de la altura de la vegetación, y en última instancia conduce a una estimación errónea de los indicadores forestales relacionados con el modelado de la biomasa aérea. La precisión de los modelos digitales derivados de LiDAR dependen de tres fuentes de error ( $\mathrm{Su} \&$ Bork, 2006; Bater \& Coops, 2009): a) errores asociados con el sensor laser, con los sistemas de navegación y con el posicionamiento durante la adquisición de datos; b) incertidumbres geométricas relacionadas con la altitud de vuelo, con el ángulo de escaneo, o con la topografía local; y c) errores que surgen en la etapa de post-procesamiento, tales como la clasificación de puntos o la interpolación de la superficie. Es importante tener en cuenta estas 3 fuentes de error en el momento de ejecutar un proyecto con LiDAR, y minimizarlos en la medida de lo posible. Generalmente, las empresas dedicadas a prestar servicios de toma de datos LiDAR, 
consideran los 2 primeros aspectos en el momento de planificar la misión de vuelo. De esta manera aseguran la integridad de los datos que van a ser entregados al cliente. La tercera fuente de error ha de ser tenida en cuenta por el usuario final de los datos.

\subsubsection{Modelo Digital de Superficie (MDS)}

El MDS representa la superficie topográfica del terreno que incluye edificios, carreteras, vegetación, es decir todos los elementos ya sean naturales o artificiales presentes en el terreno. Disponer de un MDS completo y preciso a partir de los datos LIDAR, ha proporcionado a los silvicultores un nivel de información detallada raramente disponible antes del desarrollo de esta tecnología. La información adicional acerca de la estructura vertical de la vegetación, está teniendo un gran impacto en la gestión forestal.

La técnica más fácil para obtener el MDS, es calcular una red triangular irregular o TIN con los retornos láser más altos, es decir tomando el punto más elevado dentro de una zona concreta, e interpolar usando diferentes técnicas de interpolación de superficie. EL MDS suele calcularse utilizando el primer retorno y el MDT con el último retorno (Hyyppä et al., 2009). Para garantizar que no se producirán errores sistemáticos entre el primer y último retorno, la calibración del sensor se realiza en lugares planos, sin vegetación, tales como caminos, pistas de aterrizaje, techos, campos deportivos. Se puede encontrar información sobre los métodos de interpolación en Felicísimo (1994).

\subsection{Estimación de biomasa sobre el suelo usando LiDAR}

Los enfoques típicos para predecir AGB con datos LiDAR se basan en modelos de regresión que relacionan las métricas LiDAR con estimaciones de biomasa de parcelas de inventario forestal. Estos modelos se obtienen a partir de un estudio estadístico de detalle que garantice su consistencia y su rigor matemático, así como su capacidad predictiva, de tal forma que, el modelo calculado pueda ser utilizado para estimar AGB sobre áreas más grandes. La figura 2.9 esquematiza los procedimientos utilizados para determinar los modelos de estimación de variables o parámetros forestales con un enfoque basado en métricas obtenidas con LiDAR (Zhao et al., 2011). 


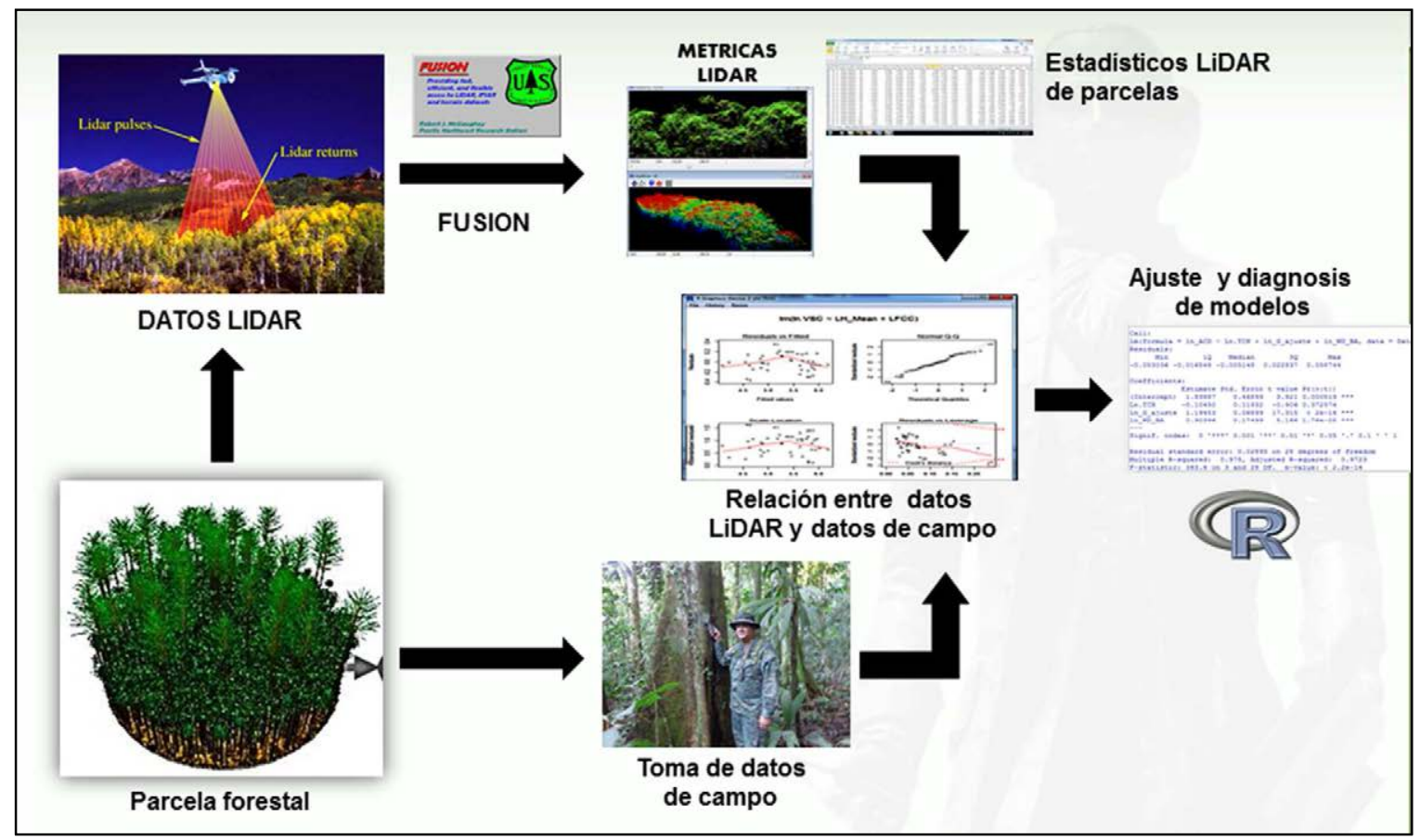

Figura 2.9: Flujo de trabajo para la caracterización de variables forestales con un enfoque basado en métricas obtenidas con LiDAR; Adaptado de K. Zhao et al. (2011).

Existe una extensa bibliografía sobre la estimación de parámetros forestales en bosques templados usando LiDAR. Säynäjoki et al. (2008) analizaron 34 estudios realizados en países nórdicos sobre el uso de datos LiDAR para inventario forestal. Los parámetros analizados fueron: área de estudio, veracidad de los datos de campo, métodos aplicados, modelos de regresión resultantes y la fiabilidad de los mismos. Aunque la revisión de la literatura se limita a bosques templados en los países nórdicos, no obstante, es una fuente importante de información para entender los aspectos claves a considerar en un inventario con LiDAR. Así mismo, Zolkos et al. (2013) realizaron un análisis detallado de las precisiones de las estimaciones de biomasa publicadas en más de 70 artículos utilizando diferentes plataformas (aéreas y espaciales), y diferentes tipos de sensores, con un enfoque particular en sistemas LiDAR. En el estudio encontraron diferencias sistemáticas en la precisión entre los diferentes tipos de sistemas LiDAR montados sobre diferentes plataformas, y lo más importante, las diferencias entre los tipos de biomas, y los tamaños de parcela utilizados para la calibración y validación de los modelos.

El mayor desafío al estimar variables biofísicas de la vegetación en bosque tropical a partir de LiDAR, es la diversidad de formas de los troncos de los árboles en la masa forestal, creando una gran cantidad de relaciones entre el LiDAR y las variables biofísicas de interés. 
Se deben identificar los criterios adecuados para relacionarlos, caso contrario, las propiedades y características de ciertos tipos de bosques pueden ser sobre-estimados mientras otros podrían ser sub-estimados. La precisión de las estimaciones de la biomasa tanto a nivel de árboles individuales como a escala de parcela, depende fundamentalmente de la exactitud de las mediciones de la altura del árbol (Hunter et al., 2013).

\subsubsection{Ecuaciones alométricas para estimar AGB en bosques tropicales}

Las existencias de biomasa aérea contenida en la vegetación por lo general se infieren a partir de los datos de inventario de campo. Las mediciones biométricas de árbol se convierten en valores de biomasa utilizando un modelo o ecuación alométrico empírico (Brown, 1997). Sin embargo, la calidad de estos modelos alométricos representa una de las limitaciones más importantes en la evaluación de las existencias de AGB, especialmente en bosques tropicales (Chave et al., 2004; Skole et al., 2011).

El desarrollo y evaluación de modelos alométricos para estimar biomasa dependen generalmente de la disponibilidad de datos de cosechas destructivas. El método más directo para estimar la biomasa sobre el suelo de un árbol implica una serie de pasos (Walker et al., 2011) que incluyen: a) cosechar el árbol, b) cortar el árbol, incluidas las hojas, ramas y tallo, en pedazos pequeños más manejables, c) secar estos pedazos en un horno y d) pesar cuidadosamente estos pedazos una vez que estén totalmente secos y que toda el agua haya sido eliminada. Obtener una verdadera medida de la AGB requerirá, en última instancia, la tala de todo el terreno de la biomasa forestal, trabajos que son extremadamente laboriosos, consumen mucho tiempo y son enormemente caros, por lo que rara vez se realizan. Las limitaciones asociadas con los métodos directos han llevado a que numerosos investigadores desarrollen ecuaciones alométricas, que relacionan la biomasa sobre el suelo de árboles individuales con otras características de los árboles que son fácilmente medibles en el campo. Estas características incluyen el diámetro a la altura del pecho (DAP), altura total y densidad de la madera. A pesar de su importancia, las ecuaciones existentes se suelen encontrar dispersas en institutos de investigación, administraciones forestales, empresas madereras y bibliotecas locales.

A menudo se argumenta que se deben calcular ecuaciones alométricas locales en tantos lugares y para el mayor número posible de especies. Sin embargo, debido a la no 
disponibilidad de esta información y la enorme variabilidad de especies, se utilizan ecuaciones alométricas pantropicales. Tal es el caso de los bosques tropicales, en donde 1 ha de bosque puede albergar entre 200 y 300 especies de árboles (Gentry, 1992; De Oliveira \& Mori, 1999), e inclusive podrían alcanzar más (Fauset et al., 2015; Valencia et al., 1994), por lo tanto, no se pueden usar ecuaciones alométricas para especies específicas como en las zonas templadas. En los últimos años se han desarrollado modelos alométricos pantropicales fiables (Chave et al., 2005, 2014; Feldpausch et al., 2011, 2012) que pueden utilizarse para predecir la AGB a través de una amplia gama de bosques tropicales.

\subsubsection{Escalamiento alométrico de parcelas agregadas}

Los bosques tropicales son muy variables en su estructura, arquitectura, y composición florística, y la influencia de estas propiedades ecológicas sobre la eficacia de LiDAR para el cartografiado de carbono, no ha sido evaluado en un amplio rango de tipos de bosques y composiciones (Asner et al., 2012a). Tres factores estructurales básicos describen la cantidad de carbono almacenado por encima del suelo en todos los bosques: el área basal (BA), la altura (H) y la densidad de la madera (WD).

Sin embargo, hasta la fecha, la información ecológica básica de la alometría alturadiámetro y la densidad de la madera a nivel de masa, no ha sido incorporada de manera directa en los métodos de cartografiado de carbono forestal a escalas regional y global. La incorporación de estos patrones estructurales de los bosques puede reducir de forma considerable el tiempo requerido para calibrar los datos LiDAR aerotransportados con los datos de parcelas de inventario forestal, trabajos que en la actualidad se llevan a cabo con mediciones exhaustivas de diámetros y alturas.

En un estudio realizado por Asner et al. (2012a), encontraron que las relaciones entre las alometrías simples a nivel de parcela y las métricas obtenidas de LiDAR aerotransportado no son consistentes a través de los ecosistemas tropicales. Para hacer frente a esa variación, propusieron un enfoque general de escalamiento alométrico de parcelas agregadas. El escalamiento alométrico de parcelas agregadas propone que, si la estructura del bosque y la organización de la biomasa siguen patrones de escala constantes, utilizar variables simples a nivel de parcela, permitirían capturar tanta información acerca de la AGB como con los trabajos de inventarios completos de campo. En esa propuesta se enfatiza que las reservas de 
carbono a nivel de árbol dependen de su diámetro, de la densidad de la madera y de la altura, y que, a nivel de parcela, la densidad de carbono sobre el suelo debe depender de manera similar del área basal, de la densidad de madera ponderada por el área basal, y, de la altura media del dosel en la parcela. La teoría propuesta de escalamiento alométrico de parcelas agregadas, sigue la teoría general de alometría de árbol (Chave et al., 2005), y refleja las propiedades de la estructura forestal de la parcela completa en conjunto, en lugar de las propiedades de cada organismo en particular.

La alometría “ideal” de árbol para una especie viene dada por la ecuación 2.4:

$$
\mathrm{AGB}=\mathrm{aD}^{\mathrm{b}}
$$

Donde AGB es la biomasa arbórea sobre el suelo, D es el diámetro del tronco (cm), a y b son los parámetros a estimar empíricamente, la variable b es el coeficiente de escalamiento alométrico (Niklas, 2006).

La forma de esta función de potencia ha sido fundamental en la teoría alométrica de árbol durante casi un siglo (West et al., 1999). Sin embargo, la alta diversidad biológica de los bosques tropicales, la variación entre especies (por ejemplo, la densidad de la madera, la forma del árbol, y la altura relativa alcanzada por unidad de incremento en diámetro), conduce a un rendimiento más débil de la función de potencia para la alometría de árbol tropical (Brown, 1997; Chave et al., 2005). Por el contrario, las ecuaciones alométricas generalizadas se adaptan a múltiples especies, y son más robustas a la disminución de la biomasa en relación con el diámetro de los árboles de mayor edad (Chave et al., 2005), e incorporan términos como el diámetro, la altura y la densidad de la madera. Juntas, estas 3 variables explican aproximadamente el 95\% de la variación en el logaritmo de la biomasa de árbol. La forma más simple de la ecuación viene representada por (ecuación 2.5):

$$
A G B=a D^{b 1} * H^{b 2} * W D^{b 3}
$$

Donde D es el diámetro del tronco (cm), H es la altura del árbol y WD es la gravedad especifica de la madera o densidad de madera $\left(\mathrm{g} \mathrm{cm}^{-3}\right)$. 
A partir de la ecuación 2.5 de Chave et al., (2005), y extendiendo la teoría de alometría de árbol individual a escala de parcela, Asner et al. (2012a) proponen el siguiente modelo general (ecuación 2.6) para la estimación de la densidad de carbono sobre el suelo (ACD) a nivel de parcela:

$$
A C D=a M C H^{b 1} * B A^{b 2} * W D_{B A}^{b 3}
$$

Donde la densidad de carbono sobre el suelo (ACD) corresponde al valor de la biomasa sobre el suelo multiplicado por el factor 0.48 (Martin \& Thomas, 2011), MCH es la altura media del perfil de dosel obtenida con LiDAR, BA es el área basal y $\mathrm{WD}_{\mathrm{BA}}$ es la densidad de la madera ponderado con relación al área basal en cada parcela.

El modelo se ajusta mediante regresión lineal múltiple de los datos a nivel de parcela transformando logarítmicamente las variables $\mathrm{ACD}, \mathrm{MCH}, \mathrm{BA}, y \mathrm{WD}_{\mathrm{BA}}$ en la forma de la ecuación 2.7:

$$
\ln (A C D)=\ln (a)+b_{1} \ln (M C H)+b_{2} \ln (B A)+b_{3} \ln W D_{B A}
$$

A continuación, se vuelve a transformar el modelo final y se multiplica por un factor de corrección (FC) para eliminar el sesgo producido por la transformación logarítmica en el modelo (Baskerville, 1972). El factor de corrección está dado por $\mathrm{CF}=\mathrm{e}^{\mathrm{MSE} / 2}$, donde MSE es el error cuadrático medio del modelo de regresión.

\subsubsection{Modelo general de estimación de ACD}

Asner et al. (2012a) propusieron un modelo universal simplificado que fue calibrado y validado experimentalmente en cuatro regiones tropicales: Perú, Panamá, Madagascar y Hawái. Este modelo explicó el 95\% de la variación de la ACD en 482 parcelas de campo utilizadas en este estudio, y quedó expresado por la ecuación 2.8:

$$
A C D=2.04 M C H^{0.436} B A^{0.946} W D^{0.912}
$$

El objetivo de esta tesis es continuar con la validación de la teoría de escalamiento alométrico de parcelas agregadas, y por consiguiente del modelo general de ACD a nivel de 
parcela propuesto por Asner et al. (2012a); evaluando si es posible su implementación en otras regiones geográficas, específicamente en el Parque Nacional Yasuní en Ecuador, o si el modelo propuesto por Asner et al. (2012a) queda relegado a las zonas de estudio de las que surgió.

\subsubsection{Fuentes de error en las estimaciones de ACD usando LiDAR}

El uso de datos LiDAR ya sea para propósitos de investigación científica o en el ámbito económico, requiere de un estudio cuantitativo de los errores asociados al cartografiado de la ACD contenida en la biomasa cuando se utiliza esta tecnología. Existen varias fuentes de incertidumbre en la estimación de la biomasa y carbono forestal. Entre las más importantes se pueden citar (Lu et al., 2012): a) muestreo sesgado, b) errores de ubicación de la parcela, c) errores en la medición de las variables del árbol (diámetro y altura), d) uso indebido de ecuaciones alométricas para el cálculo de la biomasa, e) incertidumbres de los factores de conversión entre biomasa y carbono, f) errores en la corrección geométrica y radiométrica de los datos obtenidos por teledetección, y e) la selección de los parámetros de entrada utilizados en los algoritmos de modelado. La clave para reducir estas incertidumbres es identificar las fuentes que causan dichas incertidumbres, y evaluar su incidencia en el resultado final.

Los inventarios forestales por muestreo sistemático miden un pequeño porcentaje de la superficie del bosque (fracción de muestreo), por lo que se tiene un error asociado a esa fracción de muestreo (parcela), error que se denomina habitualmente como error de muestreo. En un inventario con LiDAR se dispone de mediciones del sensor láser en toda la superficie de trabajo, por lo que la fracción de muestreo es del 100\%, y por consiguiente el error de muestreo desaparece tal y como se lo entiende en un inventario por muestreo sistemático. En un inventario LiDAR, el error está asociado a la bondad de ajuste de la regresión que relaciona las mediciones del LiDAR con las variables de inventario forestal, por lo que, conforme los modelos sean mejores, mejores serán las estimaciones. En nuestro caso, para simplificar vamos a considerar que solo es significativa esta última fuente de error y como despreciables las otras fuentes de error.

Cuando se utilizan sensores LiDAR para el cartografiado de la ACD, los errores están relacionados con el proceso de calibración, es decir con la capacidad del sensor para 
predecir la ACD de un conjunto de parcelas de inventario de campo según lo evaluado por el modelo de regresión (Mascaro et al., 2011a). Los errores en la calibración pueden ser asociados con el mapa de ACD resultante solo si la resolución del mapa (tamaño del pixel), es la misma que el tamaño de la parcela utilizada en la calibración del modelo.

Existen 3 fuentes de error que pueden influir en la relación entre las estimaciones de la ACD obtenidas con LiDAR y las estimaciones obtenidas en campo en todas las resoluciones espaciales (Mascaro et al., 2011a):

1. Errores de posicionamiento GPS.

2. Diferencias temporales entre las mediciones LiDAR y las obtenidas en campo.

3. Discrepancias entre las mediciones de campo y las mediciones con LiDAR, acerca de la posición de los árboles o parte de ellos, y si estos árboles están dentro o fuera de la parcela de calibración (efecto de borde de parcela).

Así mismo, Chave et al. (2004), cuantificaron cuatro tipos de incertidumbre que podrían conducir a errores estadísticos en la estimación de AGB en campo en bosque tropical: a) error debido a la medida de árbol; b) error debido a la elección del modelo alométrico; c) la incertidumbre del muestreo, relacionado con el tamaño de la parcela de estudio; y d) la representatividad de una red de pequeñas parcelas a través de un vasto paisaje forestal. Encontraron que la fuente más importante de error está relacionada con la elección del modelo alométrico.

\subsubsection{Influencia de la topografía y del tamaño de parcela}

La elaboración de mapas de ACD a gran escala, requieren el uso de mediciones precisas en parcelas de campo sobre extensos gradientes de clima, topografía, hidrología, suelos y diversidad biológica (Goetz et al., 2009). Hay un gran número de atributos ecológicos que varían de forma continua a través de múltiples escalas espaciales, para los que podría ser apropiado un enfoque de gradiente. Uno de los más importantes es el hábitat (Cushman et al., 2010).

Los gradientes naturales no son distribuidos al azar a través del paisaje (Loarie et al., 2009), e influyen de manera profunda en los niveles de densidad de carbono sobre el suelo 
(Saatchi et al., 2007). La mayoría de las especies de plantas se distribuyen de forma no aleatoria a través de gradientes ambientales de luz, agua y nutrientes. En los bosques tropicales, estos gradientes son el resultado de procesos biofísicos relacionados con la estructura de la cubierta forestal y la topografía (Wolf et al., 2012).

La falta de coincidencia en las escalas espaciales entre parcelas de inventario de campo y las resoluciones espaciales de los mapas que representan la biomasa forestal, usando técnicas de teledetección, merece especial atención, ya que tiene el potencial de introducir errores, sobre todo si la biomasa forestal muestra una fuerte variación espacial local (RéjouMéchain et al., 2014).

En el estudio realizado por Marvin et al., (2014) sobre el error en las estimaciones de variables forestales en campo, en tierras bajas de la Amazonía, se encontraron sesgos grandes y muy variables en la representación de la estructura del bosque y la biomasa a escala de paisaje. Los altos coeficientes de variación (CV) de las variables estimadas, revelaron heterogeneidad significativa a escala de paisaje, y por lo tanto error en el muestreo.

De la misma forma, en un estudio sobre la estructura espacial de la biomasa forestal y sus consecuencias para el cartografiado de las reservas de carbono, Réjou-Méchain et al. (2014) encontraron que la variabilidad espacial local de la ACD es grande para parcelas pequeñas ( $46.3 \%$ en parcelas de 0.1 ha), mientras que, para parcelas grandes la variabilidad es menor. Este estudio fue realizado en 30 parcelas permanentes grandes distribuidas globalmente en bosques templados, subtropicales y tropicales.

La distribución de la mayoría de propiedades forestales estructurales, así como la biomasa, es a menudo no normal con distribuciones sesgadas o multimodales (Marvin et al., 2014). Como resultado de ello, sería necesario disponer de un gran número de parcelas de campo para poder capturar la heterogeneidad de una manera estadísticamente robusta. Los resultados obtenidos por Marvin et al. (2014) y Rejou-Mechain et al. (2014), ponen de manifiesto que no se pueden obtener estimaciones precisas de biomasa a escala de paisaje como se viene realizando en la actualidad. Por lo tanto, se deben desarrollar nuevos enfoques de muestreo que reduzcan en la medida de lo posible los sesgos de las parcelas al estimar la AGB. 
La cuestión central es cómo caracterizar con precisión los paisajes forestales tropicales heterogéneos. No es posible caracterizar el paisaje y la heterogeneidad forestal a nivel regional sin el uso de datos de teledetección en esas escalas. Es esencial el uso de instrumentos de teledetección activos como LiDAR, capaces de capturar la heterogeneidad de los atributos estructurales forestales dentro de los paisajes. Una vez que se tenga una comprensión básica de la heterogeneidad espacial de un paisaje a partir de datos de teledetección, las parcelas de muestreo de campo pueden ser ubicadas de tal manera que sea posible capturar toda la variabilidad del paisaje.

A la vista de los hallazgos descritos; las estrategias de muestreo y los procesos de calibración para la determinación de la AGB usando LiDAR, deben ser analizados espacialmente. La topografía, el tamaño de las parcelas de muestreo, su representatividad y localización dentro del paisaje circundante deben ser considerados en la planificación de los inventarios forestales.

\subsection{Métodos de estimación de variables forestales con LiDAR}

Los inventarios utilizando LiDAR se pueden clasificar en 2 grupos dependiendo del tipo de variable a estimar (Hyyppä et al., 2004): inventario LiDAR de árbol individual e inventario LiDAR por métodos de masa. En los métodos de árbol individual, la información LiDAR es utilizada para buscar mediante algoritmos máximos relativos, por lo que es necesario información de la forma y dimensiones de la copa para ajustar previamente los algoritmos de búsqueda (Wang et al., 2008). A medida que aumenta la espesura de la masa forestal y la tangencia de copas, la individualización de los árboles es más complicada, por lo que son necesarias mayores densidades de puntos. En cambio, los métodos de masa buscan estimar variables dasométricas a partir de los estadísticos de la estructura de vegetación obtenidos de la información LiDAR. Los métodos de masa con LiDAR se apoyan en el ajuste de regresiones, que relacionan los estadísticos de la nube de puntos LiDAR con las principales variables de masa estimadas en campo; por ejemplo, altura dominante, área basimétrica o basal, biomasa, carbono, etc. Esta tesis se enfoca en los métodos de masa. 


\subsubsection{Estadística Inferencial}

La estadística inferencial se ocupa de los procedimientos para poder inferir o determinar las propiedades de toda la población a partir del estudio de una muestra de la misma. Además, se encarga de determinar el grado de fiabilidad o significación de los resultados obtenidos.

\subsubsection{Inferencia paramétrica}

La inferencia paramétrica comprende los procedimientos y modelos estadísticos que están basados en distribuciones de los datos reales que son determinadas usando un número finito de parámetros numéricos. Para ello es necesario conocer a priori la distribución de los datos. Para utilizar la inferencia paramétrica es necesario que la distribución de la variable respuesta sea normal. En realidad, el requisito que se utiliza es que la distribución de los errores del modelo sea normal. Si la distribución no es normal, es posible que haya que utilizarse otra familia de funciones y no se podrán usar los modelos paramétricos. Los modelos que se van a utilizar en esta tesis son los modelos de regresión.

\subsubsection{Inferencia no paramétrica}

Si consideramos que las poblaciones no son homocedásticas o bien algunas no son normales, se puede utilizar la alternativa no paramétrica. Los modelos no paramétricos se usan cuando la distribución subyacente no se ajusta a los criterios paramétricos descritos anteriormente. En definitiva, que su distribución no puede ser definida a priori. Para el análisis de una gran cantidad de variables se utiliza la técnica no paramétrica Random Forest.

\subsection{El modelo de regresión lineal múltiple}

El análisis de regresión es una técnica estadística para el modelado y que explica la dependencia de una variable con respecto de una o varias variables cuantitativas. Un modelo de regresión lineal múltiple permite relacionar una variable respuesta o dependiente $Y_{i}$ con una serie de variables explicativas o independientes $X_{i}, \ldots, X_{k}$ a partir de un conjunto de datos: 


$$
\left(y_{i}, x_{1 i}, \ldots, x_{k i}\right) \quad \text { para } i=1, \ldots, n
$$

Este modelo puede ser expresado en la siguiente forma (ecuación 2.9):

$$
Y_{i}=\beta_{0}+\beta_{1} X_{1 i}+\beta_{2} X_{2 i}+\cdots+\beta_{p} X_{k i}+\varepsilon \quad \text { para } i=1, \ldots, n
$$

Donde:

- $\quad \mathbf{Y}_{i}:$ variable respuesta o dependiente.

- $\mathbf{X}_{\mathbf{1}}, \mathbf{X}_{\mathbf{2}}, \ldots, \mathbf{X}_{\mathbf{k}}$ : variables explicativas o independientes.

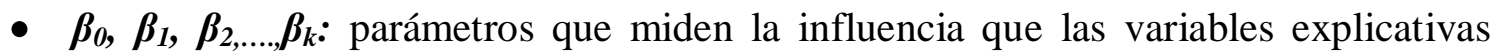
tienen sobre la variable dependiente.

- $\varepsilon$ : perturbación aleatoria (efecto adicional debido a otras variables que no se incluyen en el modelo por no ser consideradas relevantes).

\subsubsection{Hipótesis del modelo de regresión lineal múltiple}

Un modelo de regresión lineal múltiple debe cumplir con las siguientes hipótesis:

Para realizar un análisis de regresión lineal es necesario que se cumplan los siguientes supuestos:

1. Normalidad: las observaciones $Y_{i}$ siguen una distribución normal.

2. Linealidad: los valores medios de la variable respuesta dependen linealmente de los valores de $X_{1}, \ldots, X_{K}$.

3. Homocedasticidad o igualdad de varianzas: $V\left(Y_{i}\right)=\sigma^{2}$.

4. Las observaciones son independientes.

5. Ausencia de multicolinealidad: no existen relaciones lineales entre las variables explicativas $X_{1}, \ldots, X_{K}$.

\subsubsection{Rectas de Regresión}

Las rectas de regresión son las rectas que mejor se ajustan a la nube de puntos (o también llamado diagrama de dispersión) generada por una distribución binomial. Matemáticamente, son posibles dos rectas de máximo ajuste:

La recta de regresión de Y sobre X (ecuación 2.10): 


$$
y=\bar{y}+\frac{\sigma_{x y}}{\sigma_{x}^{2}}(x-\bar{x})
$$

La recta de regresión de X sobre Y (ecuación 2.11):

$$
x=\bar{x}+\frac{\sigma_{x y}}{\sigma_{x}^{2}}(y-\bar{y})
$$

La correlación ("r") de las rectas determinará la calidad del ajuste. Si r es cercano o igual a 1, el ajuste será bueno y las predicciones realizadas a partir del modelo obtenido serán fiables (el modelo obtenido resulta verdaderamente representativo); si r es cercano o igual a 0, se tratará de un mal ajuste en el que las predicciones que se realicen a partir del modelo obtenido no serán fiables (el modelo obtenido no resulta representativo de la realidad). Ambas rectas de regresión se intersecan en un punto llamado centro de gravedad de la distribución.

\subsubsection{Modelos de regresión utilizados para inventario con LiDAR}

Los modelos de regresión más habituales para realizar inventarios con LiDAR son (Agresta S. Coop, 2015):

\section{Polinomiales:}

1. Lineales (ecuación 2.12):

$$
f(x, a)=a_{0}+a_{1} x \quad a=\left(a_{0}, a_{1}\right)
$$

2. Cuadráticas (ecuación 2.13):

$$
f(a, x)=a_{0}+a_{1} x+a_{1} x^{2} \quad a=\left(a_{0}, a_{1}, a_{2}\right)
$$

3. En general de grado menor o igual a m (ecuación 2.14):

$$
f(a, x)=a_{0}+a_{1} x+a_{1} x^{2}+\ldots \ldots+a_{m} x^{m} \quad a=\left(a_{0}, a_{1}, a_{2} \ldots \ldots, a_{m}\right)
$$

Potenciales (ecuación 2.15):

$$
f(x, a)=a_{0} \cdot x^{a_{1}} \quad a=\left(a_{0}, a_{1}\right)
$$


Exponenciales (ecuación 2.16):

$$
f(x, a)=a_{0} \cdot\left(a_{1}\right)^{x} \quad a=\left(a_{0}, a_{1}\right)
$$

Existen diversos modelos que, no siendo directamente modelos lineales, admiten una transformación que los permite estudiar como si fueran modelos lineales. Los más utilizados son:

1. Modelo potencial:

Si tomamos logaritmos en la expresión de la función potencial, obtendremos la ecuación 2.17:

$$
\ln Y=\ln (a)+b \cdot \ln (X)
$$

Se obtiene la ecuación de una recta: $\mathrm{Y}=\mathrm{a}+\mathrm{bX}$, donde ahora $\mathrm{a}=\ln (a)$. El problema se reduce a transformar $\mathrm{Y}$ en $\ln \mathrm{Y}$ y $\mathrm{X}$ en $\ln \mathrm{X}$ y ajustar una recta a los valores transformados. El parámetro $b$ del modelo potencial coincide con el coeficiente de regresión de la recta ajustada a los datos transformados, y a se obtiene mediante el antilog (a).

2. Modelo exponencial:

En determinados experimentos, la dependencia entre las variables $\mathrm{X}$ e $\mathrm{Y}$ es de forma exponencial, en cuyo caso interesa ajustar a la nube de puntos una función del siguiente tipo (ecuación 2.18):

$$
y=a e^{b x}
$$

Mediante una transformación lineal, tomando logaritmos, se convierte el problema en una cuestión de regresión lineal en la forma de la ecuación 2.19:

$$
\ln y=\ln a+b x
$$

Con ello se puede ajustar el modelo transformando logarítmicamente la variable 
Y, y resolviendo el modelo lineal. A pesar de ser inicialmente modelos mucho más complejos, éstos se reducen al modelo lineal sin más que transformar adecuadamente los datos de partida.

\subsection{Comparación, selección y validación de modelos}

Durante el proceso de determinación de modelos, es común emplear diferentes expresiones matemáticas y/o distintas metodologías de ajuste para obtener la relación entre una variable dependiente y una o más variables independientes. Por tanto, es imprescindible contar con algún procedimiento que permita determinar que expresiones y/o metodologías son las más adecuadas para cumplir con los objetivos del modelo. Eso es lo que se denomina comparación y selección de modelos.

Tanto la comparación y la selección de modelos como la validación de un modelo se basan en evaluaciones cualitativas y cuantitativas. En el primer caso, el objetivo es analizar si cada ecuación o componente del modelo por separado, y el conjunto de ecuaciones o componentes que constituyen el modelo como un todo, tienen un comportamiento lógico. Por su parte, el objetivo de la evaluación cuantitativa es caracterizar los errores del modelo (diferencias entre el valor real medido y el que estima el modelo) en términos de su magnitud y distribución. La selección del mejor modelo debe tener en cuenta lo que se conoce sobre las relaciones entre la variable a explicar y la variable o las variables independientes, que se utilizará para construir un modelo coherente, a no ser que se pueda demostrar que el conocimiento inicial era erróneo (Peña Sánchez de Rivera, 2002).

\subsubsection{Criterios de selección de modelos}

Existen 2 tipos de criterios para comparar modelos. El primero considera medidas de ajuste del modelo a los datos, y el segundo medidas de su capacidad predictiva. El primer tipo de criterios es solo útil para comparar modelos con el mismo número de parámetros, ya que si aumentamos el número de parámetros mejora el ajuste. El segundo es más adecuado, y es el que se utiliza en la práctica (Peña Sánchez de Rivera, 2002).

La caracterización de los errores de la evaluación cuantitativa se basa habitualmente en el empleo de estadísticos de bondad del ajuste y en el análisis gráfico de los residuos. Para construir una medida descriptiva del ajuste global se utiliza el cociente entre la variabilidad 
explicada por la regresión y la variabilidad total. Esta medida se llama coeficiente de determinación, definido por la ecuación 2.20:

$$
R^{2}=\frac{\text { Variabilidad explicada }(V E)}{\text { Variabilidad total }(V T)}=\frac{\sum_{i=1}^{n}\left(\hat{y}_{i}-\bar{y}\right)^{2}}{\sum_{i=1}^{n}\left(y_{i}-\bar{y}\right)^{2}}
$$

Por su definición, es una medida acotada, siendo sus límites:

$$
0 \leq \mathrm{R}^{2} \leq 1
$$

Un $\mathrm{R}^{2}$ igual a 1 significa un ajuste lineal perfecto mientras que el valor cero indica la no representatividad del modelo lineal, lo que supone que el modelo no explica nada de la variación total de la variable $\mathrm{Y}$.

Por otra parte, el estadístico RMSE suele resultar útil porque está expresado en las mismas unidades que la variable dependiente, por lo que da una idea del error medio que se comete con el modelo, además de que penaliza los modelos con mayor número de parámetros, de acuerdo con el principio general de simplicidad científica. De ahí que, cuanto mayor sea el valor del coeficiente de determinación $\mathrm{R}^{2}$ y menor sea el valor del estadístico RMSE, mayor será la exactitud del modelo.

Para el análisis visual, se emplean los gráficos que representan los residuos frente a los valores estimados, y los que representan los residuos frente a las variables independientes. Sirven para comprobar las hipótesis de normalidad, homogeneidad de varianza e independencia de errores que se asumen al ajustar una relación empleando regresión por el método de mínimos cuadrados, y para verificar si existe alguna tendencia que pueda indicar una relación entre variables que no haya sido tenida en cuenta en el modelo.

Debido a que la bondad del ajuste no refleja necesariamente la bondad de una predicción futura, es recomendable realizar una validación del modelo con un conjunto independiente de datos. Generalmente cuando no se dispone de datos independientes, se dividirá la muestra en dos conjuntos y se utilizará una para el ajuste y otra para la validación, aunque raramente se proporciona información adicional en comparación con los estadísticos obtenidos directamente de los modelos ajustados con todo el conjunto de datos (Kozak, \& Kozak, 2003). 


\subsubsection{Ajuste de modelos de regresión lineal múltiples}

Conceptualmente, la única diferencia entre la regresión simple y la múltiple es que el número de variables independientes es mayor que 1. Como paso previo a la construcción del modelo de regresión múltiple se construyen los modelos de regresión simple entre la variable que se desea modelar y las distintas variables explicativas que se consideran.

Para ajustar modelos según una regresión lineal múltiple se procede de forma similar al ajuste de modelos lineales simples, salvo que, para no incluir varias variables, es recomendable tener en cuenta cuales son las variables $\mathrm{X}$ que normalmente participan en el modelo para describir una variable $\mathrm{Y}$ dada, o bien analizar la matriz de correlaciones para determinar qué posibles $\mathrm{X}$ están más correlacionadas con esta $\mathrm{Y}$.

En la regresión lineal múltiple se utiliza más de 1 variable explicativa lo que va a ofrecer la ventaja de utilizar más información en la construcción del modelo y consecuentemente va a permitir realizar estimaciones más precisas. Los coeficientes se eligen de forma que la suma de cuadrados entre los valores observados y los pronosticados sea mínima, es decir, asegurando que se va a minimizar la varianza residual.

La Regresión lineal múltiple con selección previa de variables paso a paso (stepwise) es una técnica paramétrica (es decir, que depende de la distribución de los datos). Esto significa que se requieren normalidad, homocedasticidad e independencia en los datos a fin de que se utilicen correctamente (Demšar, 2006). La selección paso a paso es la técnica de selección de variables más común en la investigación relacionada con el LiDAR. Se selecciona el mejor subconjunto de predictores mediante la introducción de variables al modelo candidato y eliminándolas de éste en varias iteraciones. El proceso termina cuando no es posible ni introducir ni eliminar más variables. 


\section{ESTUDIO DE CASO:}

\section{PARQUE NACIONAL YASUNÍ}

“El Parque Nacional Yasuní en la Amazonía puede ser el lugar con mayor biodiversidad en la Tierra”.

Bass et al. (2010) 


\section{Resumen:}

El objetivo de este capítulo es validar el uso de un modelo general de estimación de densidad de carbono sobre el suelo (ACD) en una zona de bosque tropical en Ecuador, que cuenta con alta diversidad florística, utilizando sensores LiDAR aerotransportados. Para ello, inicialmente, se realiza la descripción detallada del área de estudio, y de los datos de campo disponibles para calibrar el modelo general. A continuación, se explica todo el procedimiento utilizado para la captura y procesamiento de datos LiDAR, y se plantea una propuesta de diseño de muestreo estratificado usando productos derivados de LiDAR. Posteriormente, se analiza la distribución de ACD en el área de estudio con técnicas de modelado geoestadistico; se realiza el ajuste y validación del modelo general y se genera el mapa de reservas de ACD de la zona de estudio, y finalmente se efectúa el análisis de incertidumbre de la cartografía obtenida. 


\section{ESTUDIO DE CASO: PARQUE NACIONAL YASUNÍ}

\subsection{Introducción}

Los avances en el cartografiado del carbono forestal permiten reducir en gran medida las incertidumbres en el "presupuesto global de carbono", y así facilitar estrategias efectivas de mitigación de las emisiones como REDD+ (Reducción de Emisiones por Deforestación y Degradación de los Bosques). Si bien, la cartografía a gran escala se basa principalmente en datos de teledetección, la precisión de las estimaciones de carbono forestal depende fundamentalmente de la calidad de las mediciones de campo, y de los procedimientos de calibración (Réjou-Méchain et al., 2014).

El uso de datos LiDAR, ya sea para propósitos de investigación científica o en el ámbito político o económico, requiere de un entendimiento cuantitativo de los errores asociados con el cartografiado de carbono cuando empleamos esta tecnología. Estos errores están relacionados con el proceso de calibración, es decir con la capacidad del sensor LiDAR para predecir la densidad de carbono de un conjunto de parcelas de inventario de campo según lo 
evaluado por el modelo de regresión (Mascaro et al., 2011a).

En este capítulo se describen los datos y la metodología utilizados para calibrar y evaluar el modelo general de estimación de ACD descrito en el capítulo anterior.

\subsection{Descripción General de la zona de estudio}

La Amazonia, o también denominada Amazonía, es una vasta región de la parte central y septentrional de América del Sur, que comprende la selva tropical de la cuenca del Amazonas (Figura 3.1), y constituye el bosque tropical más extenso del mundo. La selva amazónica se desarrolla alrededor del río Amazonas y de su cuenca fluvial, y destaca por ser una de las regiones con mayor biodiversidad en el mundo. Se considera que la extensión de su cuenca biogeográfica alcanza los $6.970 .939 \mathrm{~km}^{2}$ repartidos entre ocho países, de los cuales Brasil y Perú poseen la mayor extensión, seguidos por Colombia, Bolivia, Ecuador, Guyana, Venezuela y Surinam.

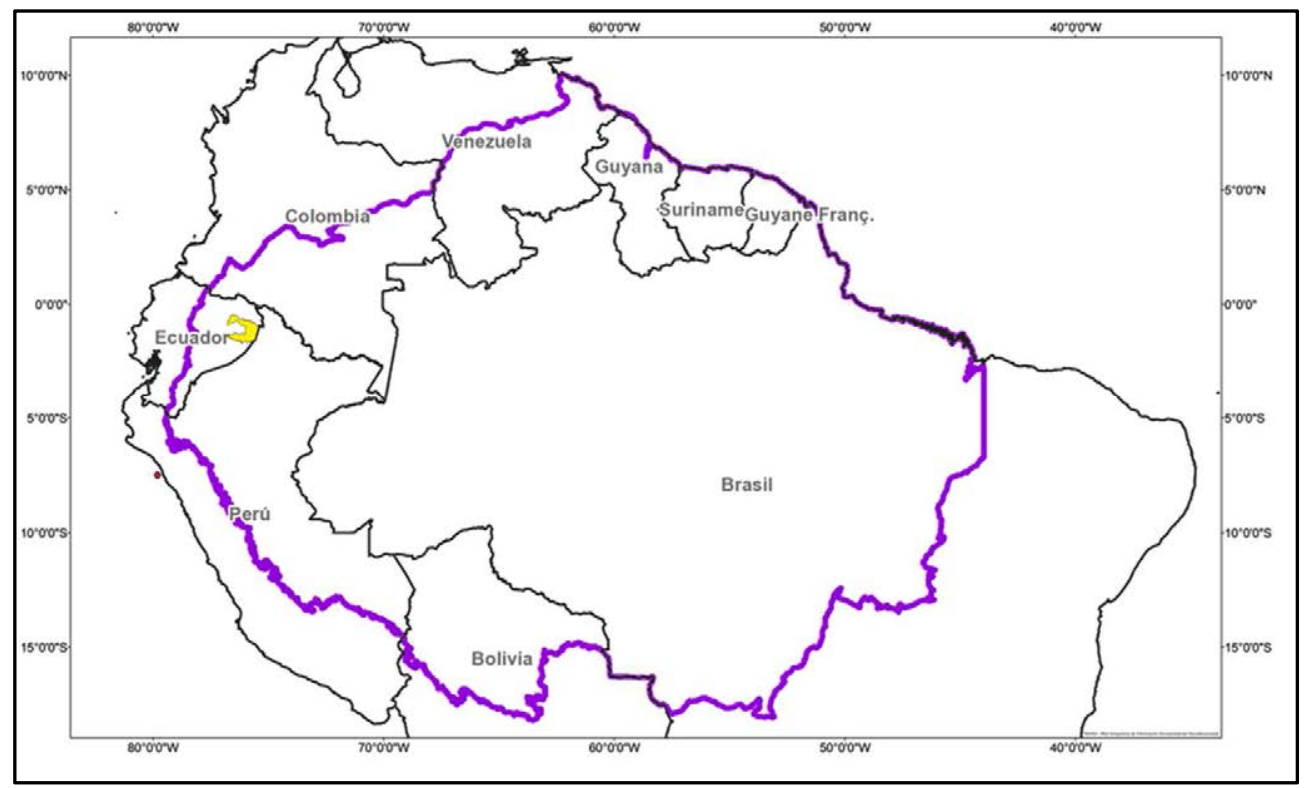

Figura 3.1: Delimitación de la cuenca amazónica (color violeta) y ubicación del Parque Nacional

“Yasuní”" (polígono amarillo); fuente: RAISG, 2015, Coordenadas geográficas en el sistema de referencia WGS-84.

Si bien es cierto que la extensión de la Amazonia Ecuatoriana, como límite biogeográfico, es pequeña en relación con la extensión total de la selva amazónica, los aproximadamente $91.071,80 \mathrm{~km}^{2}$ representan cerca del 36\% del territorio nacional (López et 
al., 2013). La ubicación geográfica de Ecuador en América del sur lo convierte en uno de los 17 países mega-diversos del mundo, con la mayor concentración de especies por unidad de área, albergando entre un 5\% y 10\% de la biodiversidad del planeta (Bass et al., 2010). En la zona nororiental de la Amazonía ecuatoriana, al sur del río Napo, se encuentra el área protegida más grande del Ecuador continental: El Parque Nacional Yasuní (1.029.566 ha). Este parque se encuentra en el sector Napo-Curaray de la Amazonía Occidental, área de estudio de esta tesis.

\subsubsection{Descripción del Sector Napo-Curaray}

Los bosques del sector Napo-Curaray de la Amazonía Ecuatoriana se ubican sobre una geomorfología que a nivel de macrorelieve pertenecen a la llanura y penillanura de la cuenca del río Napo y el norte de la subcuenca del río Curaray. Este sector se caracteriza por una estructura de colinas disectadas o muy disectadas que varían en altitud de 150 hasta 400 msnm (Pitman, 2000). En la figura 3.2 se presenta el MDT del Sector Napo-Curaray donde se encuentra localizado el Parque Nacional Yasuní, objeto de la presente investigación.

Los bosques del Parque Nacional Yasuní se caracterizan por la predominancia de suelos provenientes de aluviones andinos volcanoclásticos originados en el Mioceno, y consecuentemente con una carga más alta de nutrientes, aunque en ciertas zonas se pueden encontrar suelos con alto contenido de arena grisácea. La topografía es en general bastante accidentada lo que determina una alta variación local de la composición de sus suelos que en general son arcillosos, caoliníticos, ricos en aluminio y en bases intercambiables, aunque en ciertas zonas la textura de los suelos puede ser predominantemente limosa (Tuomisto et al., 2003). 


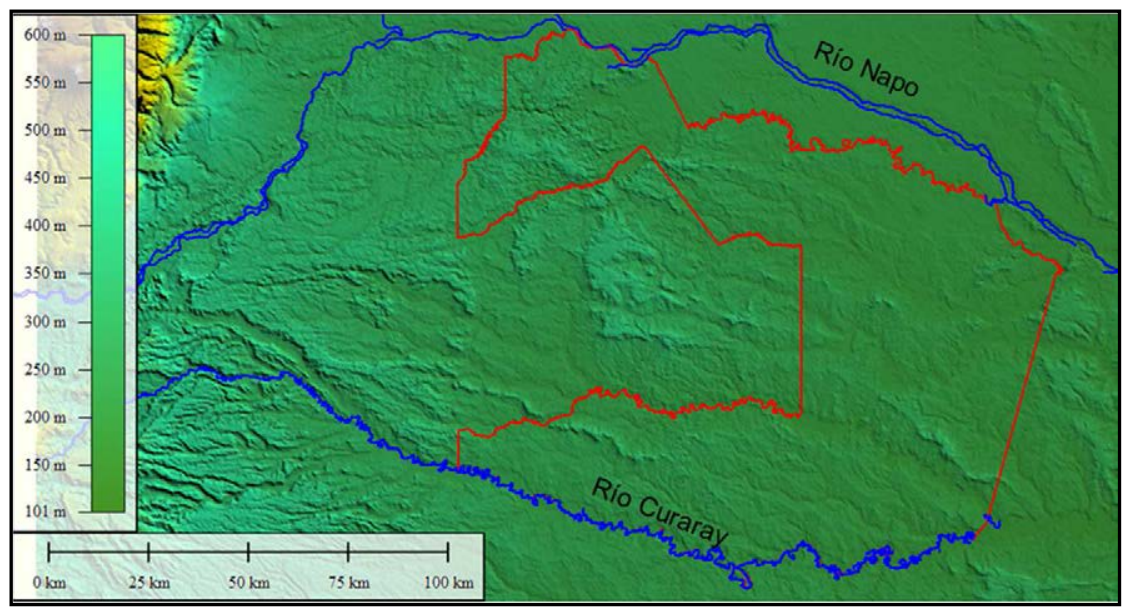

Figura 3.2: Modelo digital del Terreno del sector Napo-Curaray donde se ubica el Parque Nacional

"Yasuní" (polígono rojo); fuente: Shuttle Radar Topography Mission (SRTM) a 90 m. de resolución.

Según el sistema de clasificación de ecosistemas del Ecuador Continental (MAE,2013), la zona de estudio está localizada en el ecosistema de la Amazonía denominado "Bosque siempreverde de tierras bajas del Napo-Curaray (BsTa02)" y "Bosque inundado de la llanura aluvial de la Amazonía (BsTa09)" (figura 3.3).

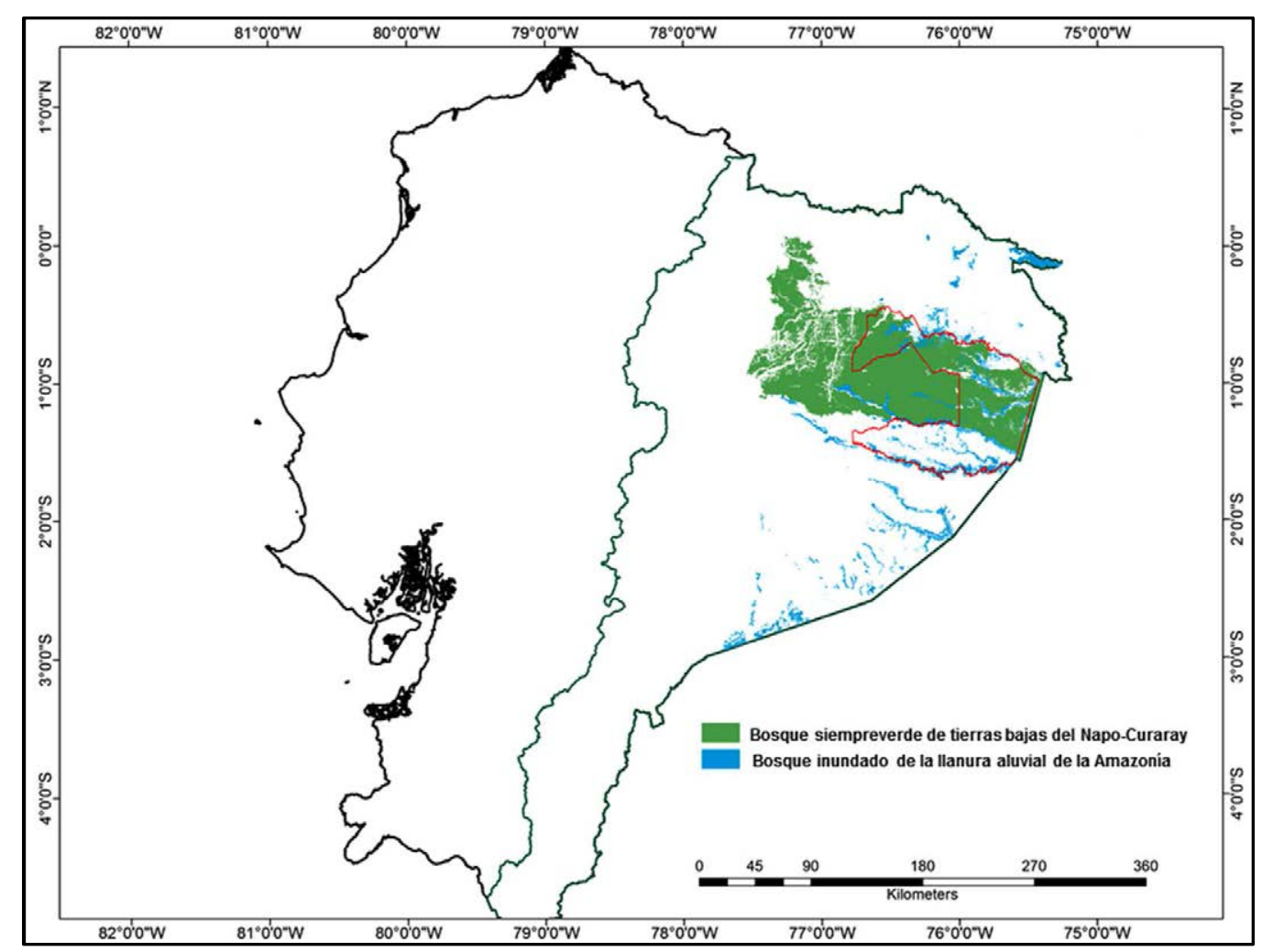

Figura 3.3: Ecosistemas en la zona de estudio; fuente: MAE (2013).

El sistema de clasificación de ecosistemas mencionado se basa en un conjunto de 
factores diagnósticos, como por ejemplo bioclima, biogeografía, geomorfología. Éstos factores sirven de apoyo para generar la leyenda de representación de los Ecosistemas del Ecuador Continental, que contiene 91 ecosistemas y grupos mayores de clasificación biogeográfica, de los cuales 43 se encuentran en la vertiente Oriental del país.

\subsubsection{Descripción del ecosistema Bosque siempreverde de tierras bajas del Napo-Curaray}

Este ecosistema incluye comunidades boscosas con gran variación en la composición, pues se trata de una de las zonas florísticamente más diversas de la Amazonía. Este sistema se desarrolla sobre áreas no inundadas (tierra firme) con relieves que varían de colinas bajas, colinas fuertemente disectadas con pendientes pronunciadas, terrazas con superficie plana y pequeños valles entre estas formaciones de orígenes sedimentarios marinos, lacustrinos y fluviales (Pitman, 2000). La topografía, bastante accidentada, determina una alta variación local de la composición de los suelos. Los bosques son principalmente siempreverdes muy altos y densos con un dosel de 30-35 m de altura con árboles emergentes de hasta 45-50 m (Valencia et al., 2004a). La tabla 3.1 resume los factores diagnósticos de este ecosistema:

Tabla 3.1: Factores diagnósticos del ecosistema BsTa02; fuente: MAE (2013).

\begin{tabular}{|l|l|}
\hline \multicolumn{2}{|c|}{ FACTORES DIAGNÓSTICOS DEL BOSQUE SIEMPRE VERDE DE TIERRAS BAJAS DEL } \\
NAPO-CURARAY (BsTa02) \\
\hline Fisonomia & Bosque \\
\hline Bioclima & Pluvial, Ombrotipo (Io): húmedo \\
\hline Biogeografía & $\begin{array}{l}\text { Región: Amazonía, Provincia: Amazonía } \\
\text { Noroccidental, Sector: Napo-Curaray }\end{array}$ \\
\hline Fenología & Siempreverde \\
\hline Piso & $\begin{array}{l}\text { Tierras bajas (170-350 msnm), termotipo (It): } \\
\text { Infratropical, termotropical }\end{array}$ \\
\hline Gioclimático & $\begin{array}{l}\text { Relieve general: Oriente, Macrorelieve: Llanura, } \\
\text { Penillanura, Mesorelieve: Colinas, Terrazas, llanura } \\
\text { aluvial }\end{array}$ \\
\hline Inundabilidad general & Regimen de inundación: no inundable \\
\hline
\end{tabular}

En este ecosistema se ha registrado la más alta diversidad de especies de árboles, así como los mayores valores de diámetros de las especies (Valencia et al., 2004b). Las familias más abundantes son: Arecaceae, Fabaceae, Moraceae, Rubiaceae, Sapotaceae, 
Melastomataceae; mientras que las más diversas son: Fabaceae, Lauraceae, Myrtaceae, Rubiaceae, Melastomataceae, Sapotaceae. (Guevara et al., 2013a).

\subsubsection{Descripción del ecosistema Bosque inundado de la llanura aluvial de la Amazonía}

Este ecosistema está conformado por un conjunto de comunidades de fisonomía muy variada, incluyendo los bosques donde se puede dar la dominancia de una o varias especies como es el caso de los bosques de Ficus spp., Coussapoa trinervia, Pachira aquatica y Machaerium floribundum, y los bosques semiabiertos de árboles asociados con palmeras hasta pantanos herbáceos arbustivos (Guevara et al., 2013b). Ocupa las llanuras de inundación en terrazas bajas o depresiones recientes y subrecientes, así como zonas hacia el interior de la llanura de inundación que se encuentran adyacentes a la matriz de tierra firme y que pueden estar rodeados por pequeñas colinas. La tabla 3.2 resume los factores diagnósticos de este ecosistema:

Tabla 3.2: Factores diagnósticos del ecosistema BsTa09; fuente: MAE (2013).

\begin{tabular}{|l|l|}
\hline \multicolumn{2}{|c|}{ FACTORES DIAGNÓSTICOS DEL BOSQUE INUNDADO DE LA LLANURA ALUVIAL DE LA } \\
AMAZONÍA (BsTa09) \\
\hline Fisonomia & Bosque \\
\hline Bioclima & Pluvial, Ombrotipo (Io): húmedo \\
\hline Biogeografía & $\begin{array}{l}\text { Región: Amazonía, Provincia: Amazonía } \\
\text { Noroccidental, Sector: Napo-Curaray }\end{array}$ \\
\hline Fenología & Siempreverde \\
\hline Piso & $\begin{array}{l}\text { Tierras bajas (158-350 msnm), termotipo (II): } \\
\text { Infratropical, termotropical }\end{array}$ \\
\hline Gioclimático & $\begin{array}{l}\text { Relieve general: Oriente, Macrorelieve: Llanura, } \\
\text { Penillanura, Mesorelieve: Terrazas, mesetas }\end{array}$ \\
\hline Inundabilidad general & $\begin{array}{l}\text { Regimen de inundación: inundable, origen de las } \\
\text { aguas: Amazónico, tipo de agua: negra }\end{array}$ \\
\hline
\end{tabular}

Las especies más representativas son: Apeiba aspera, Aspidosperma darienense, Astrocaryum urostachys, Ceiba pentandra, Chelyocarpus ulei, Coccoloba densifrons, Coussapoa trinervia, Eschweilera coriacea, Guarea guidonia, Hieronyma alchorneoides, Inga ruiziana, I. stenoptera, Iriartea deltoidea, Manilkara bidentata, M. inundata, Matisia bracteolosa, Nectandra paucinervia, Otoba parvifolia (Guevara et al., 2013b). 


\subsubsection{Parcela de investigación de la Pontificia Universidad Católica del Ecuador}

Los bosques tropicales de la Amazonía occidental concentran una inmensa diversidad de especies de árboles, una proporción significativa de estas especies tienen densidades muy bajas (Pitman, 2000). A pesar que durante décadas los botánicos han estudiado y documentado estos bosques intensamente, aún es posible descubrir nuevas especies. Debido a esta particularidad, y con el fin de investigar cómo se mantiene la megadiversidad de árboles y la extrema rareza de sus especies, en 1995 la Pontificia Universidad Católica del Ecuador (PUCE), el Instituto Smithsoniano de Estudios Tropicales y la Universidad de Aarhus establecieron una parcela de 50 hectáreas en el noroeste del Parque Nacional Yasuní en $0^{\circ} 41^{\prime}$ de latitud Sur y $76^{\circ} 24^{\prime}$ longitud oeste, justo al Sur del rio Tiputini (figura 3.4). La parcela está asociada a una red mundial de grandes parcelas permanentes (AndersonTeixeira et al., 2015) dedicadas al estudio de la función de los bosques tropicales y templados y a su diversidad.

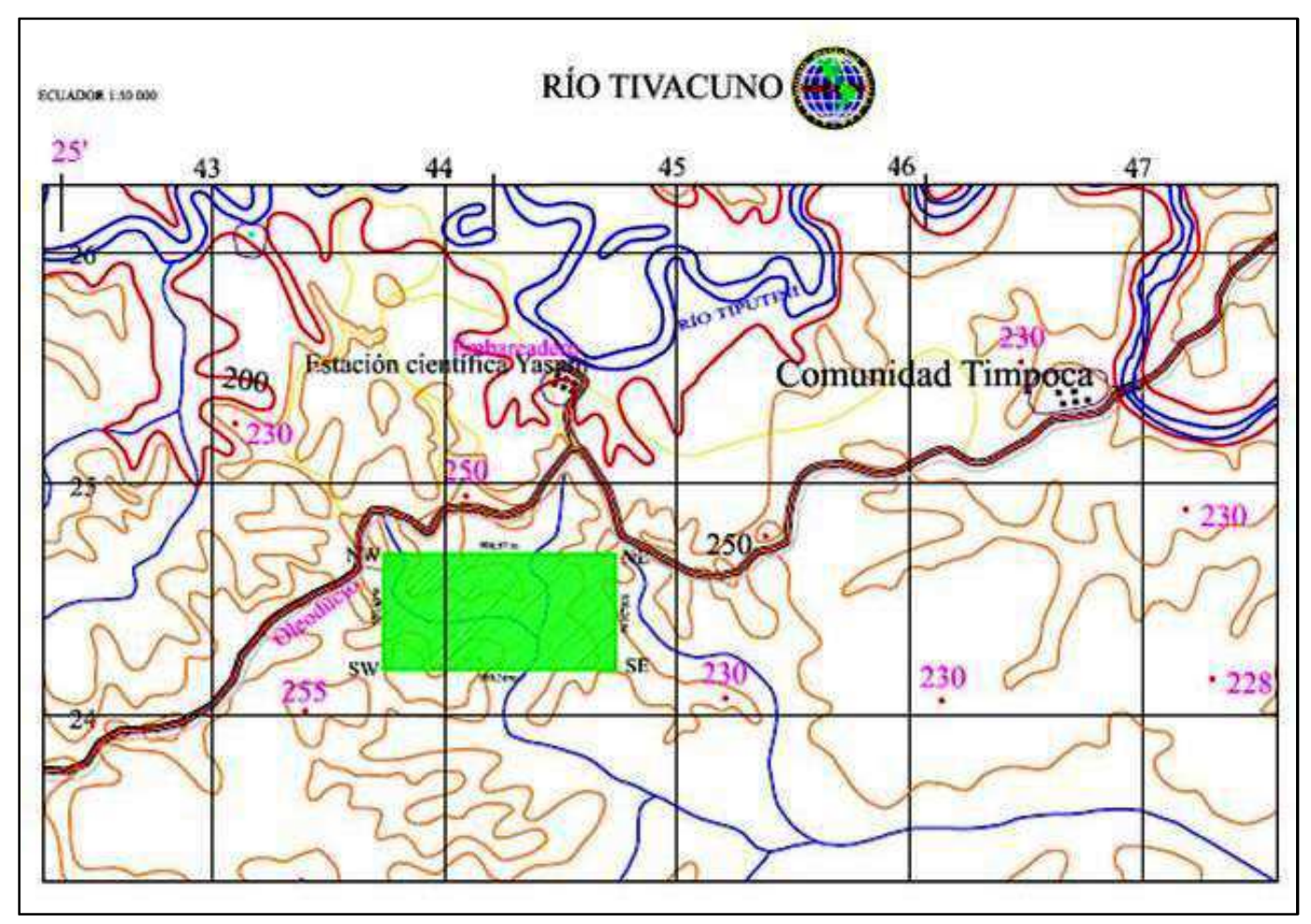

Figura 3.4: Ubicación de la parcela de investigación de 50 hectáreas (color verde); Mapa base: Carta topográfica "Rio Tivacuno”, Esc. 1:50.000, IGM, UTM 18 S-SIRGAS. 


\subsubsection{Topografía y suelos}

La parcela de 50 ha está localizada entre los 215 y 249 metros sobre el nivel del mar con una pendiente media del 13\%. En la parcela se encuentran tres crestas, compuestas principalmente por colinas de pendiente suave, y con presencia de pendientes pronunciadas a lo largo de las cárcavas (figura 3.5). Las partes bajas incluyen pequeños arroyos permanentes y pantanos (frecuentemente inundados) en la parte este de la parcela (Valencia et al., 2004a). Dentro de la parcela se encuentran 2 hábitats topográficos característicos y dominantes: valle y colina. En el valle se encuentra un pantano (1,7 ha) asociado a una depresión donde crece la palma Mauritia flexuosa, y un parche de bosque secundario $(0.48$ ha) dominado por Cecropias ciadophylla, y que corresponde a un antiguo helipuerto que fue abierto en la época de prospección petrolera en la zona, alrededor del año 1987.

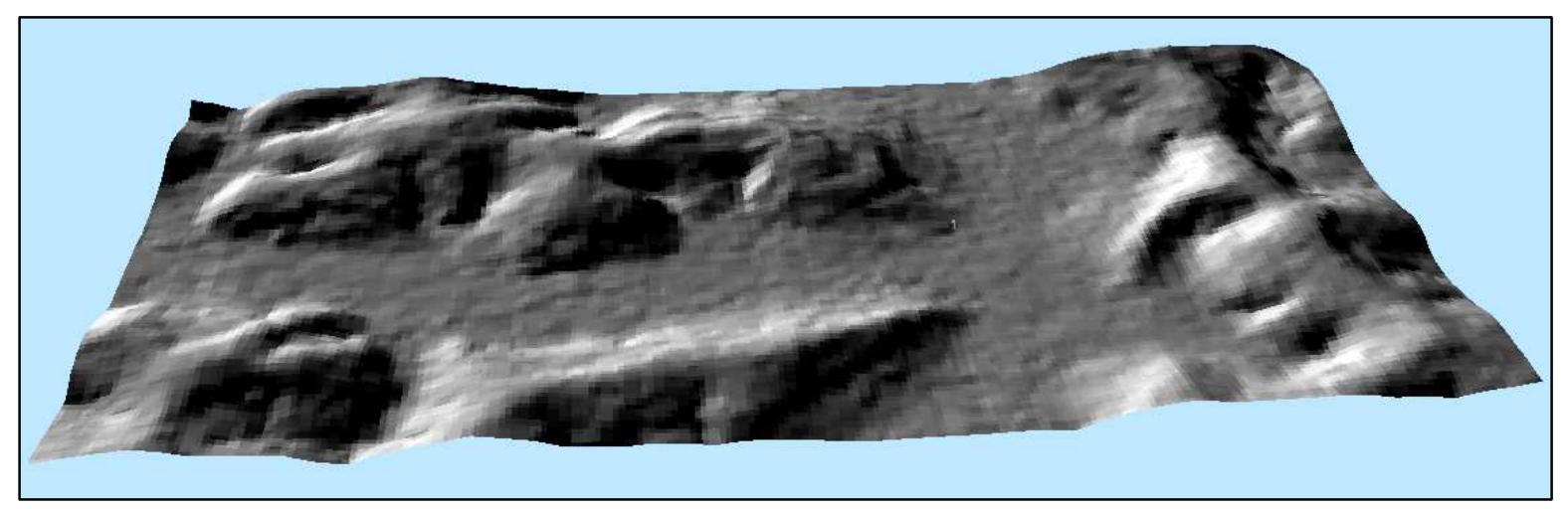

Figura 3.5: Mapa de sombras de la parcela de estudio generado a partir de datos LiDAR.

En las partes elevadas, los suelos son arcillosos, ácidos, y ricos en aluminio y hierro (Pitman, 2000). La mayoría de los suelos en el Parque Nacional Yasuní se clasifican como Ultisoles, mientras que, en las partes bajas, propensas a inundaciones, predominan los Histosoles. En un estudio realizado por John et al. (2007) sobre la influencia de los nutrientes del suelo en la distribución espacial de las especies de árboles tropicales en el Parque Nacional Yasuní, encontraron que un porcentaje de la distribución espacial de las especies $(51 \%)$ muestran fuertes asociaciones a las distribuciones de nutrientes del suelo. De la misma forma, Baldeck et al. (2013) evidenciaron que los recursos del suelo, por ejemplo, $\mathrm{Al}, \mathrm{Ca}, \mathrm{Cu}, \mathrm{Fe}, \mathrm{K}, \mathrm{Mg}, \mathrm{Mn}, \mathrm{P}, \mathrm{Zn}, \mathrm{pH}$; y la topografía, explicaron el 9,6\% de la variación de la estructura comunitaria local de los árboles; y se concluyó que existe una gran similitud entre la diversidad beta y el mapa topográfico del lugar. 


\subsubsection{Tipo de Bosque}

El bosque de la zona de estudio corresponde a un bosque maduro húmedo de tierras bajas y de hoja perenne, con una altura de dosel aproximada de 15 a 30 metros, con presencia de árboles emergentes que alcanzan los 40 y rara vez los 50 metros (Valencia et al., 2004a). En un estudio sobre la distribución de especies arbóreas y la variación del hábitat en 25 hectáreas de la parcela, Valencia et al. (2004b) encontraron 1104 morfoespecies entre 152.353 individuos. En el mayor número de especies de árboles, el dosel era de tamaño medio, con una altura de 10 a 20 metros, mientras que los árboles pequeños, en el sotobosque, alcanzaban una altura máxima de 5-10 m. Varias especies de árboles pequeños del sotobosque en los géneros Matisia y Rinorea dominaban el bosque numéricamente, mientras que las especies más importantes de dosel fueron Iriartea deltoidea y Eschweilera coriacea. Se pueden encontrar información más detallada sobre las características taxonómicas y ecológicas de las especies arbóreas en la parcela en Romero-Saltos et al. (2014a).

Es importante mencionar que esta parcela de campo de 50 hectáreas se encuentra localizada en 2 ecosistemas en la Amazonía ecuatoriana: bosque siempre verde de tierras bajas del Napo-Curaray y bosque inundado de la llanura aluvial de la Amazonía, y constituye una muestra aleatoria de la variación estructural de los bosques a escala de paisaje en éstos 2 ecosistemas en la amazonia occidental. Esta característica es importante, ya que para capturar la relación entre las mediciones con LiDAR y la estructura forestal de la zona de estudio, se requiere un muestreo uniforme a lo largo del rango de todas las posibles condiciones estructurales que sean comunes o poco frecuentes, así como los hábitats presentes. De ahí que, la parcela de 50 hectáreas puede considerarse una muestra adecuada para este tipo de estudios. La figura 3.6 ilustra la parcela de estudio en 3dimensiones con transferencia de color RGB de la ortofotografía. 


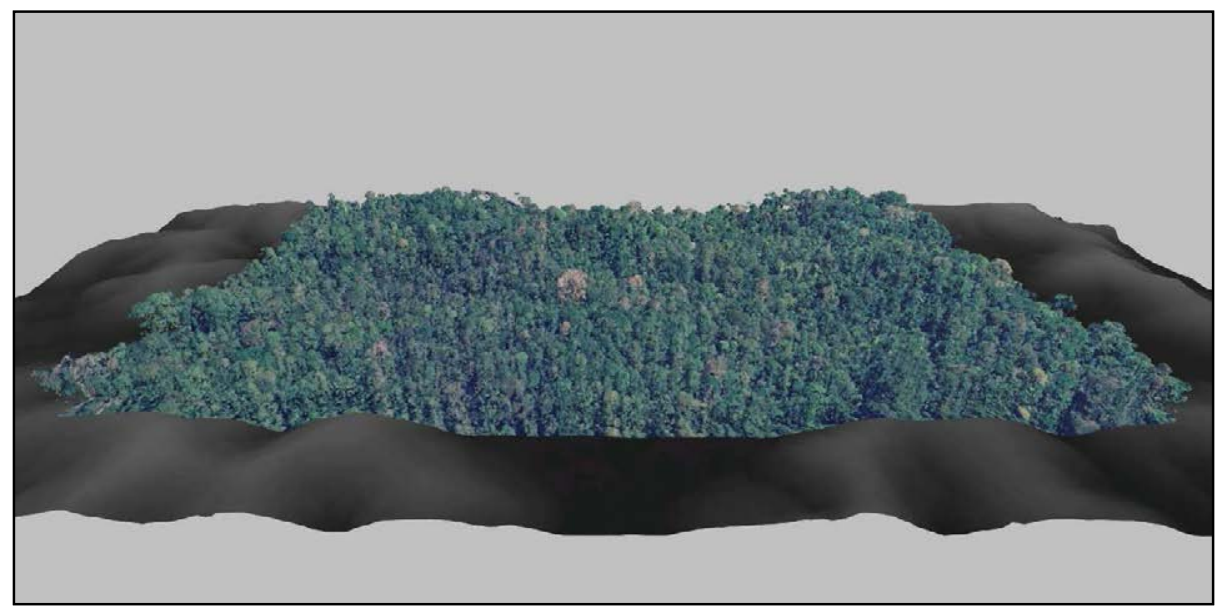

Figura 3.6: MDT con la nube de puntos LiDAR con color RGB de la ortofotografía.

\subsection{Fuentes de datos}

La estimación de parámetros forestales utilizando LiDAR requiere contar con datos LiDAR y con información de campo proveniente del levantamiento de las parcelas. En este estudio, se utilizó la información disponible del censo de árboles realizada por el Dr. Renato Valencia en esta parcela de 50 hectáreas, y se planificó un vuelo LiDAR sobre la parcela de estudio.

\subsubsection{Censo forestal en la parcela}

Los censos en la parcela son fundamentales para el estudio de la dinámica de la inmensa diversidad de árboles en la zona (Romero-Saltos et al., 2014b). El primer censo forestal en las 25 hectáreas occidentales de la parcela se realizó entre 1995 y 2000. En la parcela de 50 hectáreas se han realizado varios censos en diferentes fechas y lugares (anexo A), siguiendo un método estándar utilizado por la red mundial de grandes parcelas forestales (Condit, 1998). Debido a limitaciones económicas, el primer censo completo en las 50 hectáreas se realizó en el lapso de aproximadamente 4.3 años, desde mediados de 2002 hasta fines de 2006. Para este estudio, se utilizaron mediciones de los árboles $\geq 1 \mathrm{~cm}$ de diámetro a la altura de pecho (DAP), provenientes de este censo completo, y que suman un total de 297.777 individuos.

\subsubsection{Determinación del tamaño de muestra para el cálculo de la biomasa}

Para realizar estudios de la dinámica del bosque en entornos tropicales es necesario 
establecer parcelas de gran tamaño (Condit et al., 2000). Por otro lado, el tamaño y forma de una parcela puede variar dependiendo del tipo de datos que se están recolectando. Cuando el objetivo es realizar estimaciones de carbono y biomasa forestal, las parcelas de muestreo deben ser lo suficientemente grandes para que incluyan cualquier variabilidad local en cuanto al tipo y densidad de los árboles existentes. De ahí que se prefiere parcelas más grandes, por ejemplo 100 m x 100 m, a parcelas más pequeñas (Walker et al., 2011). Al utilizar parcelas de gran tamaño en general se produce una disminución de errores, porque se considera que en las parcelas grandes se captura una cantidad adecuada de la variabilidad estructural en el campo (Chave et al., 2004).

Una compilación de investigaciones publicadas acerca de las estimaciones de biomasa sobre el suelo usando LiDAR (Zolkos et al., 2013), concluyó que la incertidumbre de las estimaciones se acerca al 10\% cuando el tamaño de la parcela se aproxima a 1 ha. Las estimaciones de biomasa sobre el suelo a escalas espaciales por debajo de 1 hectárea están dominadas por la incertidumbre, debido a los efectos de corte de parcela, y por las diferencias en las características de los sensores (Mascaro et al., 2011a). Se admite que escalas espaciales mayores, típicamente del orden de 1 ha, dan resultados más precisos (Meyer et al., 2013). En un estudio reciente en tierras bajas de la Amazonia occidental, Marvin et al. (2014), encontraron que un tamaño de muestra promedio de 44 parcelas, de 1 ha de tamaño, permite estimar la ACD de forma fiable (es decir, con una probabilidad de 0,9) para un paisaje entre 100 y 10.000 hectáreas.

A la vista de los hallazgos de las investigaciones antes mencionadas en relación a la influencia de la estructura y variabilidad de la AGB en bosques tropicales en general (Réjou-Méchain et al., 2014), y en la zona de estudio en particular (Valencia et al., 2009); el diseño de muestreo para el análisis fue un cuadriculado regular de subparcelas de 1 ha de superficie (figura 3.7). Este cuadriculado permitió capturar el rango de variación de la estructura forestal en la zona de estudio. 


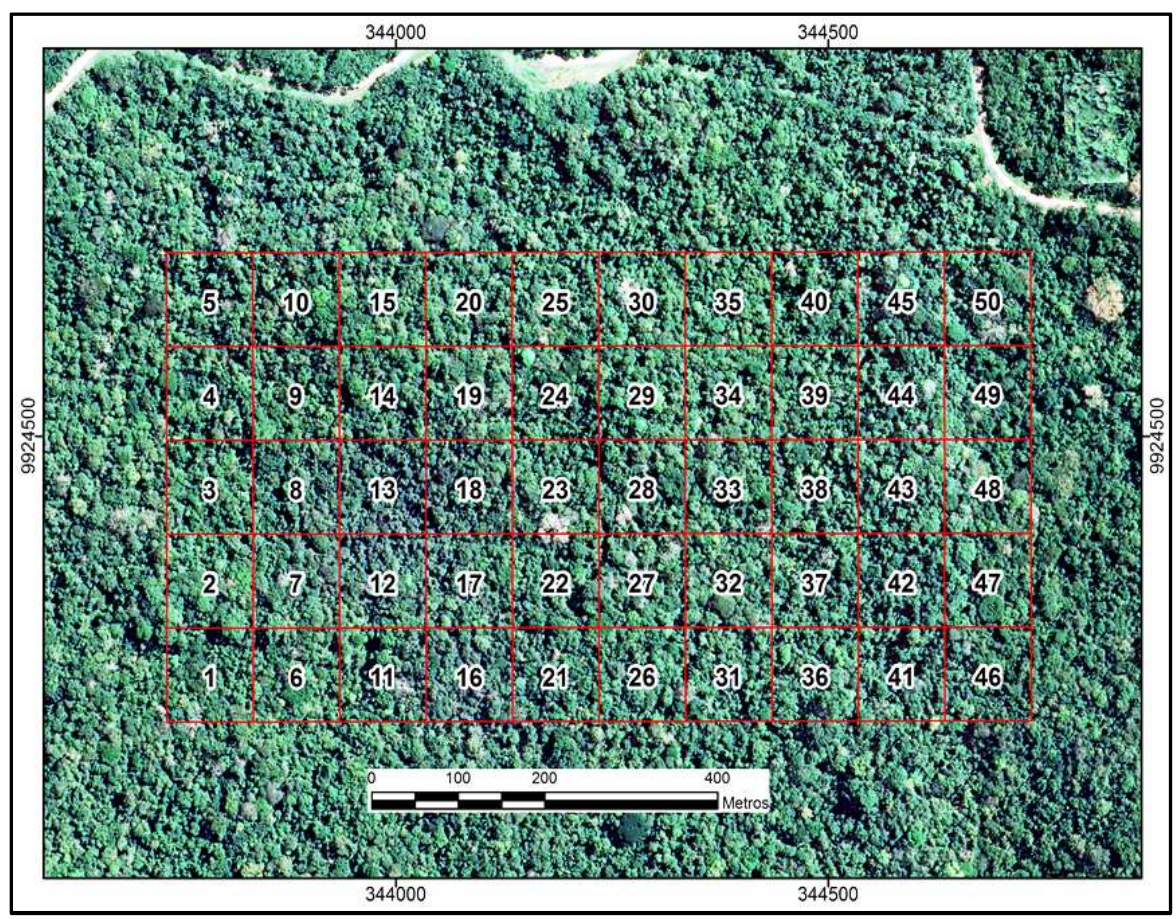

Figura 3.7: Sistema de cuadriculado regular de subparcelas de 1 ha, utilizado para el cálculo de la biomasa sobre el suelo; Fuente: ortofotografía IGM.

\subsubsection{Estimación de biomasa sobre el suelo usando modelos generales pantropicales}

Las estimaciones de biomasa de los bosques tropicales están limitadas por el conocimiento de la alometría de árboles. En general, la extrema diversidad de especies, impide que se desarrollen alometrías de especies específicas al igual que en zonas templadas, por lo que generalmente se utilizan ecuaciones generales pantropicales. La precisión para el cálculo de las reservas de carbono depende de la disponibilidad de modelos alométricos fiables, que permitan inferir la biomasa aérea de los árboles a partir de datos de inventario forestal (Chave et al., 2004, 2014; Ngomanda et al., 2014).

Para la estimación alométrica de la biomasa aérea en el área de estudio, se aplicó el modelo mejorado de Chave et al. (2014). Este modelo (ecuación 3.1) se usa en casos en los que no se cuenta con mediciones de la altura total de los árboles, e incorpora variables como diámetro del tronco, densidad de la madera, y la variable $E$. Este modelo mejora las predicciones de biomasa obtenidos con un modelo propuesto anteriormente por el mismo autor (Chave et al., 2005), y que ha sido muy utilizado en estudios de estimación de AGB en bosque tropical. 


$$
A G B_{i}=\exp \left[-1.803-0.976 E+0.976 \ln (\rho)+2.673 \ln (D)-0.0299[\ln (D)]^{2}\right]
$$

Donde D representa el diámetro del tronco a la altura de pecho en $\mathrm{cm}$, y $\rho$ es la densidad de madera en $\mathrm{g} \mathrm{cm}^{-3}$ de cada árbol.

La relación genérica entre el valor de biomasa sobre el suelo y las características forestales del área de estudio, depende linealmente de una variable de estrés bioclimático $(E)$, que a su vez depende de índices de variabilidad de temperatura, del índice de precipitaciones, y de la intensidad de la sequía.

Con los datos del censo forestal, y el valor del parámetro $E$ en el Parque Nacional Yasuní (-0.0228121), se calculó la cantidad de biomasa sobre el suelo (en unidades de $\mathrm{Mg} \mathrm{ha}^{-1}$ ), de todos los individuos existentes en la parcela. La densidad de madera o gravedad específica se obtuvo de la revisión de la literatura especializada u obtenida a partir de mediciones directas en los alrededores de la parcela o en áreas del Parque Nacional Yasuní. La sumatoria de las estimaciones de cada árbol en cada subparcela de 1 hectárea (ecuación 3.2), dio como resultado el valor total de AGB para cada una de las subparcelas:

$$
A G B_{\text {subparcela }}=\sum_{1}^{n} A G B_{i}
$$

Se realizaron estimaciones de AGB para todos los árboles de la parcela (297.777 individuos) con DAP $\geq 1 \mathrm{~cm}$, dando un total de $12.512,59 \mathrm{Mg}$. Para el ajuste del modelo general de estimación de ACD con LiDAR se consideraron sólo los árboles con DAP $\geq 10$ cm (34.567 individuos), dando como resultado un total de 11.438,68 $\mathrm{Mg}$ para la parcela completa. Generalmente los árboles $<10 \mathrm{~cm}$ de DAP, contribuyen con menos del 5\% de la AGB en los bosques tropicales maduros (Chave et al., 2003). Los resultados de las estimaciones en campo muestran que los árboles con DAP $\geq 10 \mathrm{~cm}(11,6 \%$ de todos los árboles de la parcela), contribuyen aproximadamente con el $91 \%$ del total de la AGB, mientras que la vegetación con diámetro $<10 \mathrm{~cm}$ contribuye con un $9 \%$ del total de la AGB. También se evidenció que la AGB difiere hasta en un 100\% en una variación altitudinal de tan sólo $30 \mathrm{~m}$. La variación de AGB (161 a $339 \mathrm{Mg} \mathrm{ha}^{-1}$ ) en un pequeño rango de elevación advierte una fuerte influencia de la posición topográfica. Este es el primer hallazgo 
importante en la presente tesis doctoral, ya que ratifica los resultados obtenidos anteriormente por Valencia et al. (2009), y en particular las diferencias en AGB entre valle y colina; y donde sugiere que la ecuación 3.1 produce estimaciones robustas de AGB. Sin embargo, para minimizar el sesgo en las estimaciones de AGB en campo, se aconseja siempre que sea posible, el desarrollo de relaciones de diámetro-altura derivadas localmente.

\subsubsection{Estimación del área basal en las parcelas}

El área basal (BA, basal area) o área basimétrica $(\mathrm{G})$ es la relación existente entre la suma de las superficies de las secciones normales de los árboles medidas a 1.3 metros del suelo (DAP) de una determinada masa forestal, expresadas en $\mathrm{m}^{2}$, y la superficie del terreno $\left(\mathrm{S}_{\mathrm{T}}\right)$ que ocupan, expresada en hectáreas (ecuación 3.3). Al igual que para la biomasa aérea, se calculó el área basal para cada una de las subparcelas usando la siguiente expresión:

$$
G_{S U B P A R C E L A}=\frac{\sum g_{i}\left(m^{2}\right)}{S_{T}(h a)}
$$

Donde $g_{i}=\left(\frac{\pi}{4}\right) * d_{i}^{2}$; d es el diámetro a la altura de pecho.

Los resultados de las estimaciones de biomasa sobre el suelo, área basal y densidad de madera de los individuos con DAP $\geq 10 \mathrm{~cm}$ en cada una de las 50 subparcelas se presentan en el anexo B.

\subsubsection{Geoposicionamiento y levantamiento planimétrico de la parcela}

Para estimar variables dasométricas a partir de datos LiDAR es necesario que los datos de campo obtenidos directamente sobre el terreno y los datos LiDAR, estén correctamente georreferenciados. De esta manera garantizamos que todas las fuentes de datos coincidan espacialmente (Mauro et al., 2013). A menudo los errores en la identificación y la baja precisión de las variables estimadas, son atribuidos a discrepancias entre el co-registro de las parcelas de referencia en tierra y la información LiDAR (Frazer et al., 2011). Cuando la georreferenciación de las parcelas se realiza de forma exclusiva mediante técnicas GNSS, es importante considerar el hecho de que en entornos forestales dichos equipos suelen funcionar de una forma no-óptima. El dosel arbóreo es un obstáculo que tiende a atenuar y 
distorsionar la señal GPS, por lo que la precisión de las medidas GNSS es menor dentro de bosques que en áreas abiertas (Mauro et al., 2011).

En esta tesis doctoral se realizó un levantamiento planimétrico para determinar con precisión submétrica las coordenadas de las esquinas de la parcela, y de esta forma relacionar la información del censo forestal, con los datos obtenidos a partir del vuelo LiDAR. Para el levantamiento planimétrico se implantaron 4 vértices geodésicos (NW-1, NW-2, NE-1, NE-2), utilizando tecnología GNSS, que conformaron la red básica de los trabajos de campo (figura 3.8):

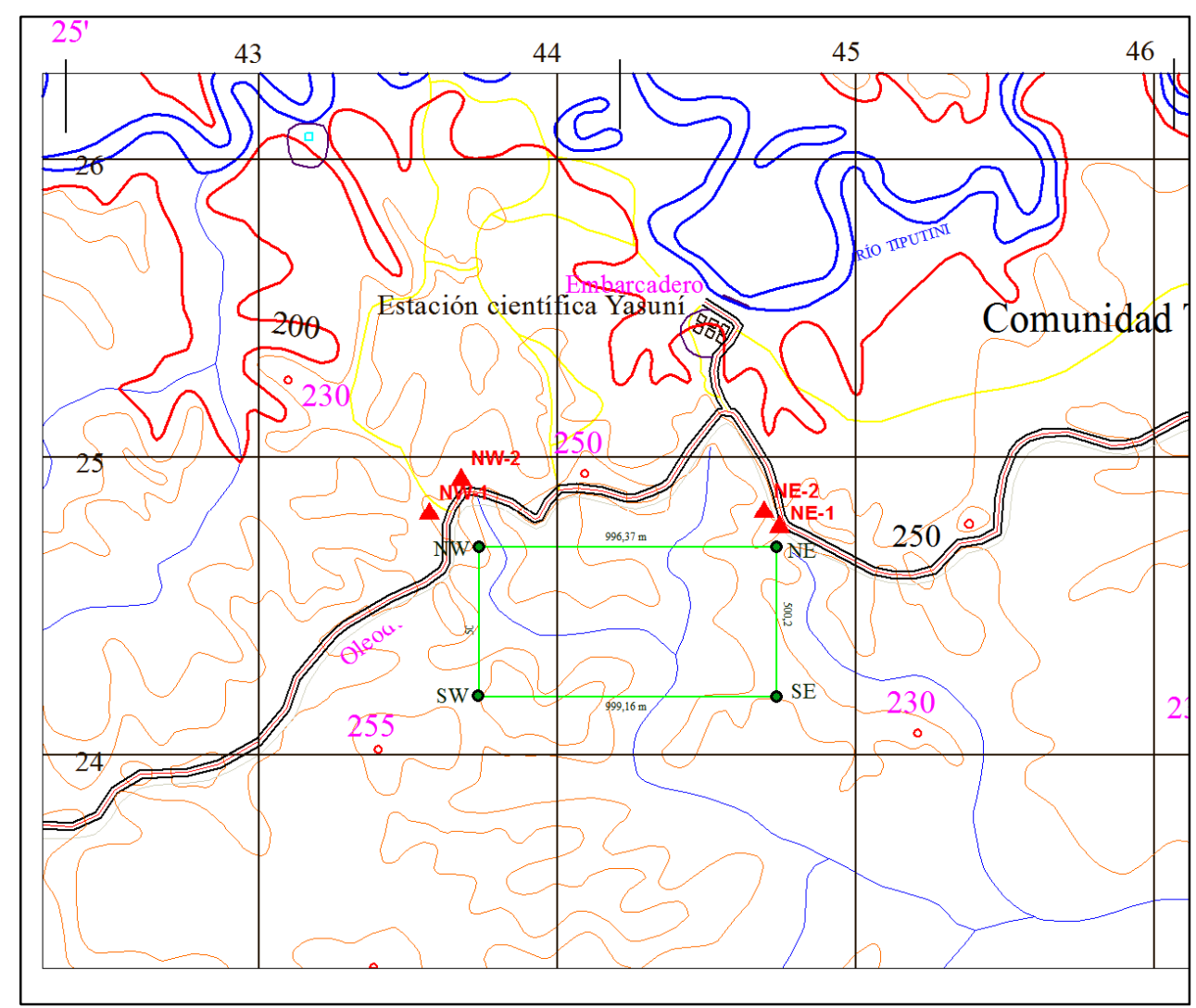

Figura 3.8: Ubicación de los 4 puntos GNSS y las 4 esquinas de la parcela; Mapa Base: C.T Río Tivacuno, 1:50.000, IGM, UTM 18 S-SIRGAS.

Para el ajuste de la red básica se incluyeron observaciones desde el vértice geodésico GNSS “Y-NPF”, que forma parte de la Red Nacional GPS del Ecuador (RENAGE). La red RENAGE se encuentra enlazada al sistema de referencia SIRGAS (Sistema de Referencia Geocéntrico para las Américas) - ITRF94 (International Terrestrial Reference Frame), época de referencia 1995.4; compatible con el sistema de posicionamiento satelital GNSS (SIRGAS., 2015). 
La determinación de las coordenadas de los vértices geodésicos se realizó por el método de posicionamiento GPS estático diferencial de fase, utilizando receptores doble frecuencia marca TRIMBLE, modelo R8, receptores que permiten la medida de líneas base hasta 200 kilómetros, con una precisión de $\pm 0.005 \mathrm{~m}+1$ ppm. Para garantizar en cada sesión GPS una correcta recepción de la información satelital, se establecieron los siguientes parámetros de observación (tabla 3.3):

Tabla 3.3: Parámetros de recepción y posicionamiento de los 4 vértices geodésicos.

\begin{tabular}{|c|c|}
\hline Tiempo de recepción mínima & 1 hora \\
\hline Angulo de enmascaramiento & $10^{\circ}$ \\
\hline $\mathrm{N}^{\circ}$ satélites mínimos enganchados & 4 \\
\hline Intervalo de grabación & 15 segundos \\
\hline Horas de recepción óptimas & PDOP menor que 5 \\
& HDOP menor que 5 \\
\hline Tipo de posicionamiento & Estático \\
\hline
\end{tabular}

Para el procesamiento de los datos GNSS se utilizó el software de procesamiento TRIMBLE BUSINESS CENTER (TBC) Versión 2.5, actualización del software GPSurvey. Para el procesamiento de cada vector GPS, se fijó la precisión horizontal de $<0.050$ m. +1 ppm, precisión vertical $<0.100 \mathrm{~m} .+1 \mathrm{ppm}$. Tras el procesamiento se obtuvieron las coordenadas de los 4 vértices observados que se presentan en la tabla 3.4:

Tabla 3.4: Coordenadas UTM ZONA 18 Sur (SIRGAS) de las bases observadas (líneas base)

\begin{tabular}{ccccc}
\hline Puntos GNSS & Este (m) & Norte (m) & Prec. (m) & Alt. Elip. \\
\hline NW-1 & 343569.287 & 9924809.647 & 0.010 & 261.540 \\
NW-2 & 343676.761 & 9924923.710 & 0.023 & 258.266 \\
NE-1 & 344744.991 & 9924763.519 & 0.016 & 259.048 \\
NE-2 & 344691.828 & 9924814.856 & 0.018 & 255.556 \\
\hline
\end{tabular}

Posteriormente se realizaron las observaciones topográficas necesarias para calcular las coordenadas de las 4 esquinas de la parcela (tabla 3.5) a partir de la red básica geodésica 
implantada.

Estas observaciones se realizaron con una estación total TRIMBLE modelo S3, con una precisión de 2".

Tabla 3.5: Coordenadas UTM ZONA 18 Sur (SIRGAS) de las esquinas de la parcela de estudio.

\begin{tabular}{ccccc}
\hline Esquinas-parcela & Este (m) & Norte (m) & Prec. (m) & Alt. Elip. \\
\hline NW & 343737.794 & 9924696.411 & 0.050 & 249.31 \\
SW & 343735.023 & 9924196.086 & 0.050 & 252.792 \\
NE & 344733.958 & 9924695.236 & 0.050 & 244.899 \\
SE & 344734.085 & 9924195.180 & 0.050 & 252.074 \\
\hline
\end{tabular}

Usando como punto de partida las coordenadas de la esquina $\mathrm{SW}$, fueron georreferenciados todos los árboles del censo, y éstos datos fueron incorporados a una base de datos geográfica. Hay que señalar la dificultad que implica realizar este tipo de levantamientos en zonas con topografía irregular, vegetación densa y en condiciones climáticas adversas, como es el caso de la zona de estudio (figura 3.9):

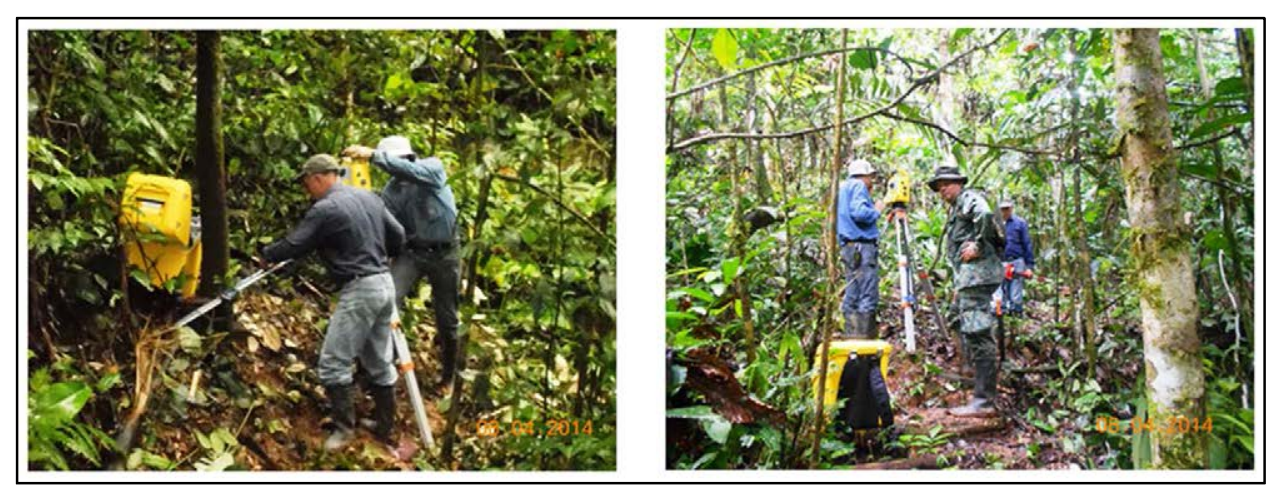

Figura: 3.9: Levantamiento planimétrico de la parcela de estudio.

\subsection{Captura de datos LiDAR de la zona de estudio}

Previo a la recopilación de datos de inventario forestal en campo, se recomienda disponer de productos provenientes del procesamiento de los datos LiDAR, como por ejemplo, MDT, MDS, nMDS; ya que proporciona información valiosa para el diseño del muestreo de campo. En este estudio, se contaba ya con los datos de campo del censo de la parcela, por lo que el vuelo LiDAR fue planificado directamente sobre esta zona. Una adecuada planificación para la adquisición de datos LiDAR, permite obtener información útil para 
diferentes aplicaciones. En la figura 3.10 se resumen los procedimientos que se utilizaron para la captura, procesamiento y clasificación de los datos LiDAR para llegar a obtener los modelos digitales del terreno (MDT).

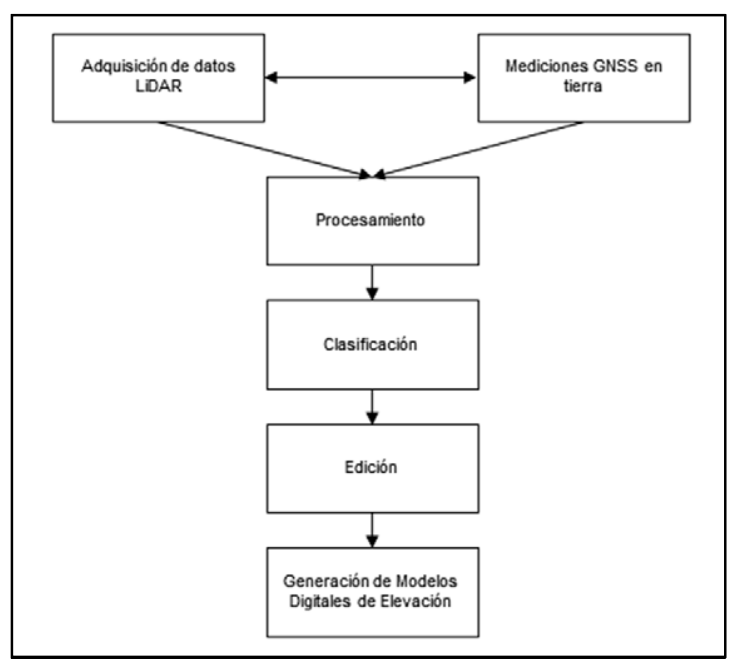

Figura 3.10: Esquema del flujo de trabajo para la generación de MDE (MDT, MDS) a partir de datos LiDAR.

\subsubsection{Planeamiento de la misión de vuelo}

Uno de los aspectos claves en la planificación del vuelo LiDAR es la determinación de la densidad de puntos a obtener. Para estudios forestales, el equipo LiDAR debe configurarse de tal forma que permita maximizar la penetración del pulso láser en el dosel arbóreo. En regiones con vegetación muy heterogénea y diversa como el Parque Nacional Yasuní, donde el estrato bajo herbáceo es bastante denso, y las epífitas, las lianas y las trepadoras son muy abundantes (figura 3.11), los sensores LiDAR producen menos retornos en tierra. De ahí que, el planeamiento de la misión LiDAR debe tener en cuenta ciertas consideraciones básicas que garanticen una adecuada penetración del pulso láser en el dosel arbóreo, y que éste alcance el suelo. Para definir éstos parámetros se analizaron estudios de biomasa en bosques tropicales con diferentes densidades de puntos; desde 25 puntos $/ \mathrm{m}^{2}$ (d'Oliveira et al., 2012), hasta densidades de 1,5 puntos $/ \mathrm{m}^{2}$ (Asner et al., 2014a). 


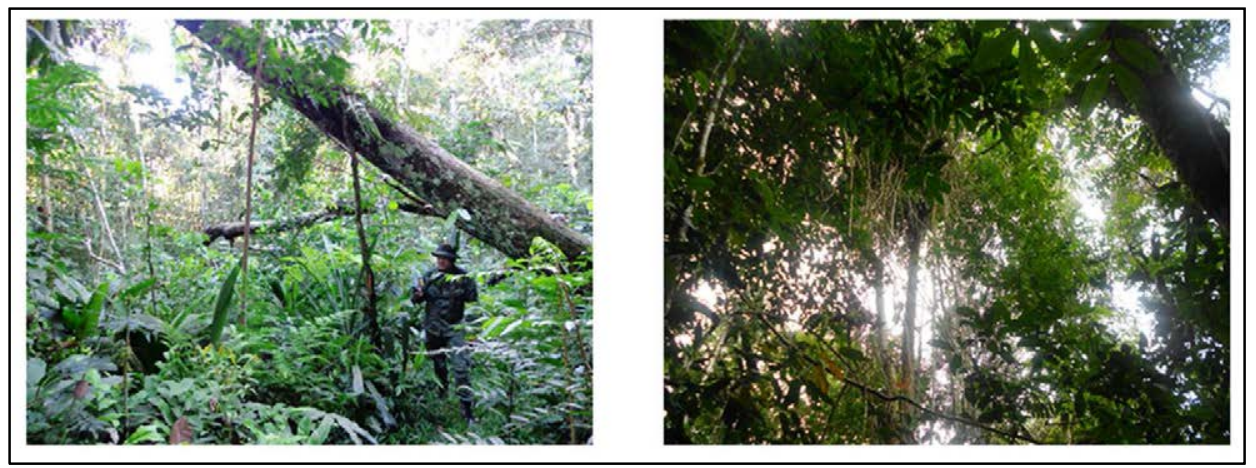

Figura 3.11: Estrato bajo herbáceo y troncos caídos (izquierda); trepadoras y lianas (derecha) en la zona de estudio.

En un estudio reciente en bosque tropical submontano y montano de la selva atlántica de Brasil, Leitold et al. (2015), se evaluaron los efectos de la densidad de puntos en la generación del modelo digital del terreno (MDT). El estudio llegó a la conclusión de que, ante una reducción en la densidad de puntos, el error sistemático en la construcción del MDT se propagó a las métricas de dosel, lo que originó en una subestimación de la biomasa forestal. Los resultados de este estudio destacan la importancia de llevar a cabo una planificación de vuelo cuidadosa y un muestreo LiDAR consistente; y concluyó que los enfoques para estimar biomasa aérea que se basan principalmente en alturas del dosel, son sensibles a los errores en el MDT debido a la variabilidad en la densidad de muestreo LiDAR.

A fin de disponer de suficientes pulsos que alcancen el suelo, y generar un MDT fiable, el vuelo LiDAR fue planificado teniendo como objetivo conseguir la mayor densidad de puntos por metro cuadrado. Usualmente se consiguen densidades de puntos altas con sistemas láser de retorno discreto, que registran retornos múltiples para cada pulso. Dependiendo del tipo de sistema de escaneo, la frecuencia, la sensibilidad para discriminar los pulsos, y el tipo de bosque o área a ser levantada, la densidad de puntos de retorno puede ser altamente variable (Chasmer \& Hopkinson, 2006; K Lim et al., 2008; Su et al., 2006). Los llamados sistemas LiDAR “topográficos” permiten un ángulo de barrido menor, lo que posibilita mejores estimaciones de altura. Este tipo de equipos LiDAR se suelen utilizar en el ámbito forestal para caracterizar masas e inventariar recursos forestales. Para esta tesis se utilizó un equipo LiDAR Optech ALTM Gemini, montado sobre una avioneta CESSNA 172 Skyhawk (figura 3.12), aeronave que permite cubrir grandes extensiones de terreno a altitudes y velocidades bajas. 


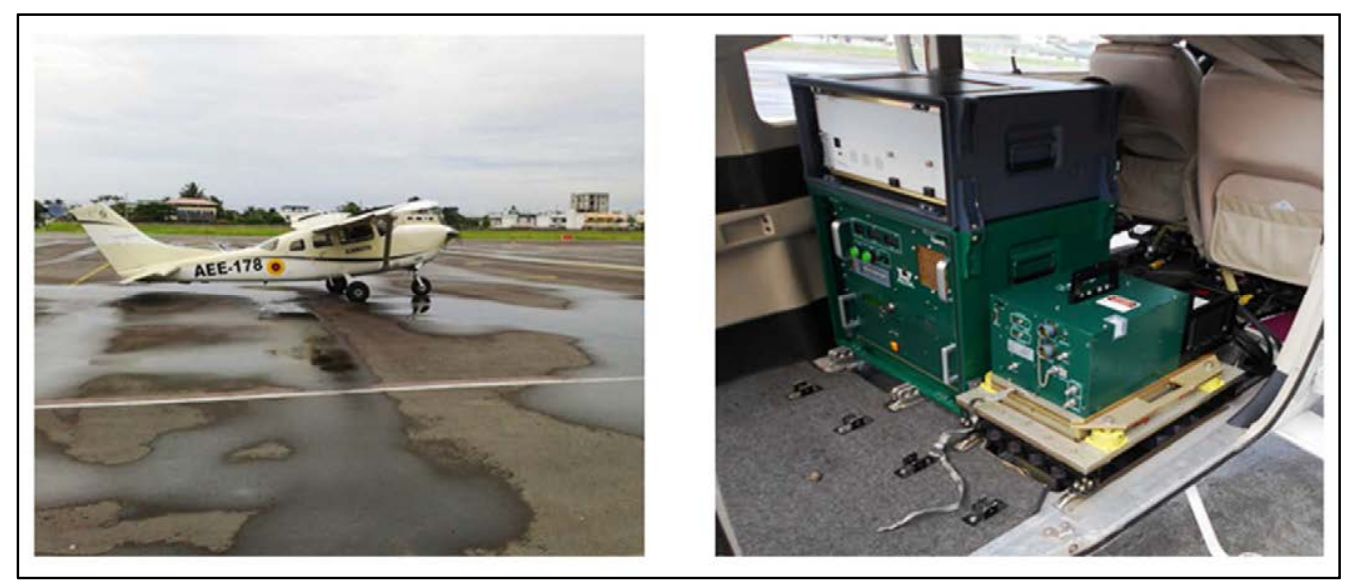

Figura 3.12: Avioneta CESSNA 172 Skyhawk (izquierda); sensor LiDAR Optech ALTM Gemini (derecha).

\subsubsection{Parámetros del levantamiento LiDAR}

Los ajustes exactos para cada levantamiento dependen principalmente de las condiciones climáticas locales, la topografía y la resolución deseada del levantamiento. Batsavias (1999), plantea una guía general de configuración de algunos parámetros para un levantamiento con LiDAR, que puede usarse para la planificación del vuelo.

Los datos LiDAR fueron obtenidos en mayo de 2014, la configuración final del vuelo y del sensor se detallan en la tabla 3.6. El área total del levantamiento con LiDAR fueron aproximadamente $40 \mathrm{Km}^{2}$ (4.000 ha), para lo cual se utilizaron $2 \mathrm{~h} 27$ min.de vuelo.

Tabla 3.6: Configuración del vuelo y del equipo LiDAR para la zona de estudio.

\begin{tabular}{|l|l|l|l|}
\hline \multicolumn{2}{|c|}{ DATOS DEL VUELO } & \multicolumn{2}{c|}{ CONFIGURACIÓN LiDAR } \\
\hline Altitud por encima del suelo (m) & 781.25 & Frecuencia de repetición de pulso (Khz) & 166 \\
\hline Superposición & $50 \%$ & Frecuencia de escaneo (Hz) & 40 \\
\hline Velocidad (m/s) & 56.6 & Ángulo de escaneo /FOV & $\pm 15^{\circ}$ \\
\hline Líneas de vuelo & 16 & Densidad nominal de pulsos por $\mathrm{m}^{2}$ & 5.08 \\
\hline Distancia entre líneas de vuelo (m) & 203.89 & Ancho de barrido (m) & 407.78 \\
\hline \multirow{2}{*}{} & & Número de retornos & Hasta 4 \\
\cline { 3 - 4 } & & Láser beam divergence (IFOV) & 0.8 \\
\cline { 3 - 4 } & & Espaciado entre puntos (m) & 0.24 \\
\cline { 3 - 4 } & & Densidad de puntos por m ${ }^{2}$ & 19.4 \\
\hline
\end{tabular}

Los parámetros que se pueden modificar en la planificación del vuelo LiDAR son: a) la configuración de vuelo, b) los ajustes del escáner laser y c) ajustes de la cámara. Estos 
parámetros se configuran de acuerdo a la densidad y el espaciamiento de puntos deseado. El espaciamiento de puntos se define como la distancia entre 2 puntos adyacentes, un diseño de proyecto óptimo, producirá espaciamientos similares entre líneas de escaneo y entre puntos dentro de la línea de escaneo. La altura de vuelo está determinada por la combinación de la velocidad de la aeronave, el ángulo de escaneo, la frecuencia de escaneo y la frecuencia de repetición de pulso.

El vuelo LiDAR fue planificado con un $50 \%$ de superposición, en cada franja y con cada pasada adyacente. La figura 3.13 ilustra la configuración que se utilizó en el plan de vuelo LiDAR en el área de estudio:

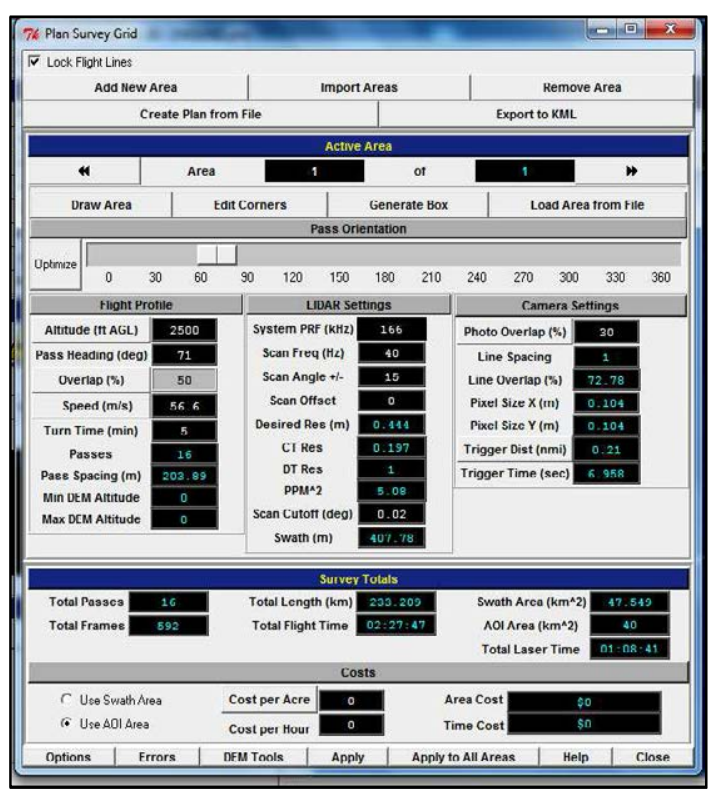

Figura 3.13: Ventana del Plan de vuelo realizado en ALTM-NAV. (Los valores en color azul son calculados automáticamente en base a los valores en blanco).

\subsubsection{Planificación de la estación de referencia en tierra}

Los proyectos de cartografía con LIDAR requieren de observaciones GNSS en tierra (referidas como estaciones base) simultáneas con el vuelo LiDAR. Estas observaciones se realizan sobre estaciones de referencia conocidas en tierra, generalmente de primer orden, en una ubicación dentro o cerca de la zona de estudio. Estas observaciones serán utilizadas posteriormente para determinar la posición exacta del avión. Configuraciones típicas para misiones con LiDAR, establecen una distancia máxima entre el receptor móvil a bordo de la aeronave y la estación de referencia de tierra dentro del rango de 10 a 50 Km. En general, 
distancias más cortas entre las estaciones terrestres de referencia y el receptor móvil en la aeronave, mejoraran la fiabilidad de la solución.

En esta investigación se utilizó la estación base LIMONCOCHA (figura 3.14), que forma parte de la Red Nacional GPS del Ecuador-RENAGE. El Sistema de Referencia Horizontal utilizado es el Sistema de Referencia Geocéntrico para las Américas (SIRGAS ECUADOR). Para las observaciones se utilizó 1 receptor geodésico GNSS marca TRIMBLE, modelo R4. La distancia entre la estación base y la zona de estudio fue aproximadamente de $30 \mathrm{Km}$.

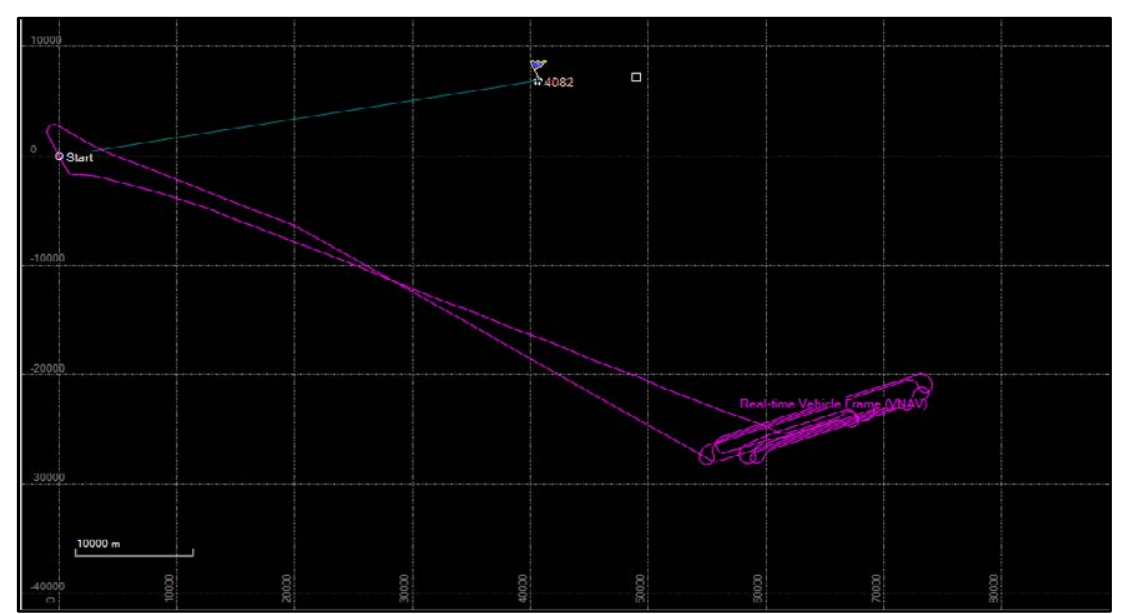

Figura 3.14: Trayectoria GPS de la misión LiDAR en la zona de estudio. La bandera azul (4082) en la imagen indica la posición de la estación base GNSS "Limoncocha".

\subsection{Procesamiento de los datos LiDAR}

Esta fase incluyó 2 actividades: el pre-procesamiento y el post-proceso de los datos LiDAR. En esta investigación, el pre-procesamiento se define como la verificación en gabinete de los datos recogidos en campo, el procesamiento de las observaciones GNSS y el procesamiento de los datos del sistema inercial (IMU); para ello se utilizaron una variedad de paquetes informáticos desarrollados y entregados por el proveedor del equipo LiDAR. El pre-procesamiento de los datos LiDAR fue realizado por personal técnico especialista del Instituto Geográfico Militar, bajo la supervisión del doctorando.

El post-proceso abarca el procedimiento completo de procesamiento de datos LiDAR que han sido calibrados y convertidos en franjas de vuelo en formato LAS (formato Standard definido por la $A S P R S$ ) en la fase de pre-procesamiento. El paso más riguroso en la etapa de 
post-proceso es la creación y/o utilización de filtros automáticos o algoritmos para la clasificación de los datos, y en concreto para la identificación de los pulsos que representan el suelo, que serán utilizados posteriormente en la creación del modelo digital de elevación. La selección del algoritmo a utilizar puede variar dependiendo de la densidad de puntos, la vegetación, el terreno, las características de los objetos ya sean naturales o artificiales, así como de la finalidad del producto resultante o de su aplicación. Un resumen detallado sobre los tipos de algoritmos y las consideraciones para utilizar determinado algoritmo se puede encontrar en Arranz (2013).

\subsubsection{Generación del Modelo Digital del Terreno (MDT)}

Para el cálculo del MDT de la zona de estudio se utilizó el algoritmo "lasground", del programa LAStools (Rapidlasso GmbH., 2013). El algoritmo utiliza una variación del método de densificación progresiva del TIN (Axelsson, 2000) que evita sobrecarga trigonométrica. Éste enfoque refina un TIN en forma adaptativa utilizando valores geométricos (distancias y ángulos) como criterios de filtro. Este algoritmo depende de 2 parámetros: a) una ventana de búsqueda (Kernel), y b) un criterio de ángulo máximo. Los puntos más bajos se consideran como suelo, y a continuación se interpola entre ellos una red triangular (TIN). El TIN se simplifica de acuerdo a los criterios seleccionados en cada iteración.

Todos los puntos de la zona de cobertura LiDAR fueron procesados para identificar cuáles fueron los pulsos que penetraron el dosel arbóreo y alcanzaron el suelo. Se seleccionó un tamaño de ventana (Kernel) de 5 metros x 5 metros, y los puntos con elevación más baja calculada en cada Kernel fueron considerados como suelo. Los demás puntos fueron evaluados ajustando un plano horizontal a cada uno de los puntos identificados como suelo (seedpoints). Si el punto no clasificado más cercano era $<5.5^{\circ}$ y menor a 1,5 metros en elevación, se clasificaba como suelo. Este proceso se repitió hasta que se evaluaron todos los puntos dentro de la cobertura de vuelo. Estos umbrales se definieron en base a los resultados obtenidos en estudios previos en bosques de similares características a los de la zona de estudio. A partir de los puntos clasificados como suelo, se generó un MDT de 5 metros de resolución con el algoritmo blas2dem como se muestra en la figura 3.15: 


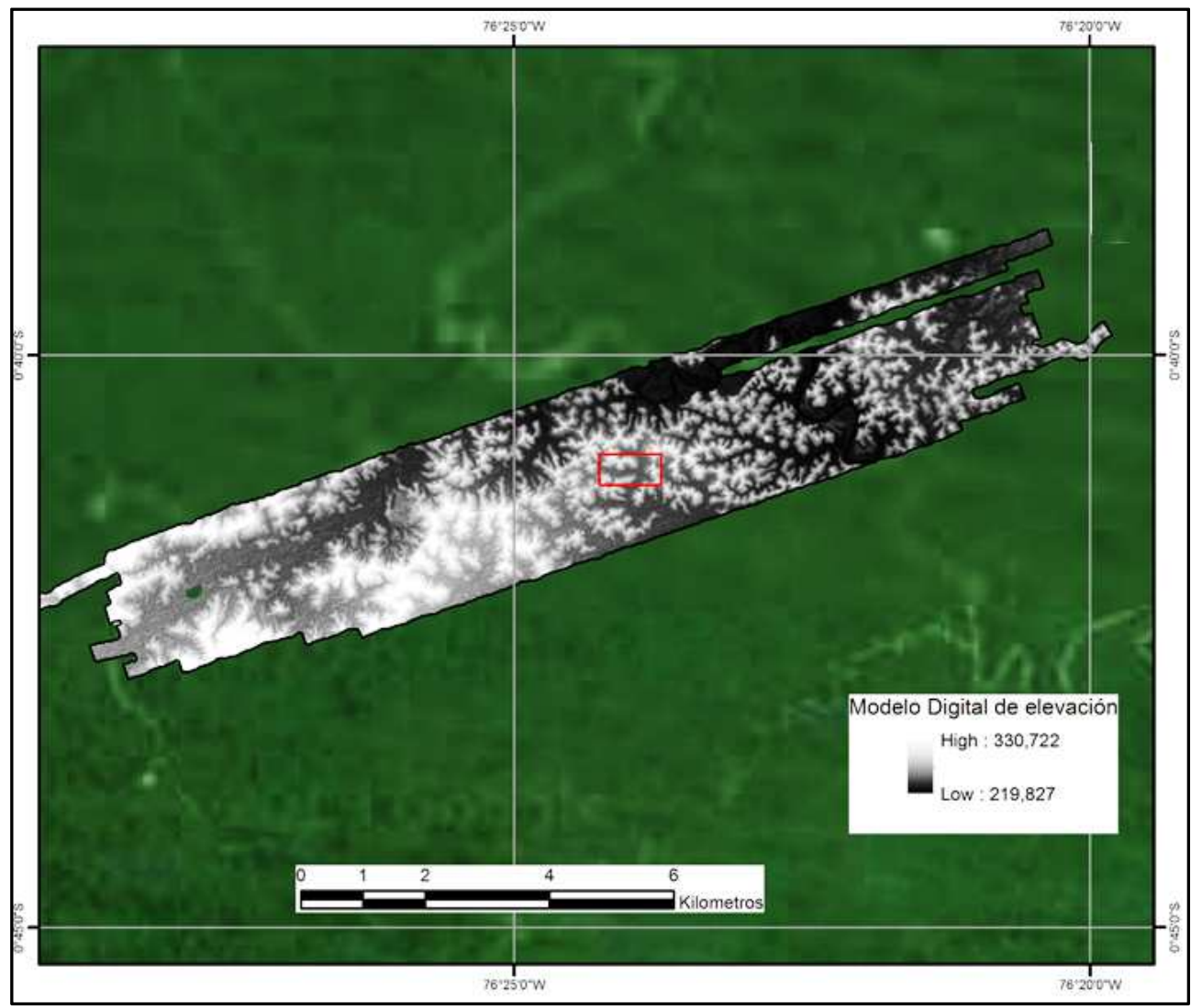

Figura 3.15: Modelo Digital del Terreno de la trayectoria de vuelo LiDAR; el rectángulo en color rojo corresponde a la ubicación de la parcela de 50-ha; imagen satelital de Google Earth.

\subsubsection{Generación del Modelo Digital de Superficie normalizado (nMDS)}

La nube de puntos LiDAR fue normalizada usando el algoritmo lasheight de LAStools (rapidlasso GmbH, 2013). Este algoritmo calcula la altura de cada punto LiDAR por encima del suelo, por lo que es necesario disponer del MDT. Utilizando la opción "replace-z", se obtiene la elevación de cada punto LiDAR con respecto al MDT.

A continuación, se generó el modelo digital de superficie normalizado (nMDS) usando blas2dem de LAStools (rapidlasso $\mathrm{GmbH}, 2013$ ), en base a la interpolación de todos los primeros retornos, y usando un step $=0.5$; obteniendo una imagen de 0.5 metros de resolución. En la figura 3.16 se presenta el modelo digital de superficie normalizado de la zona de vuelo LiDAR. 


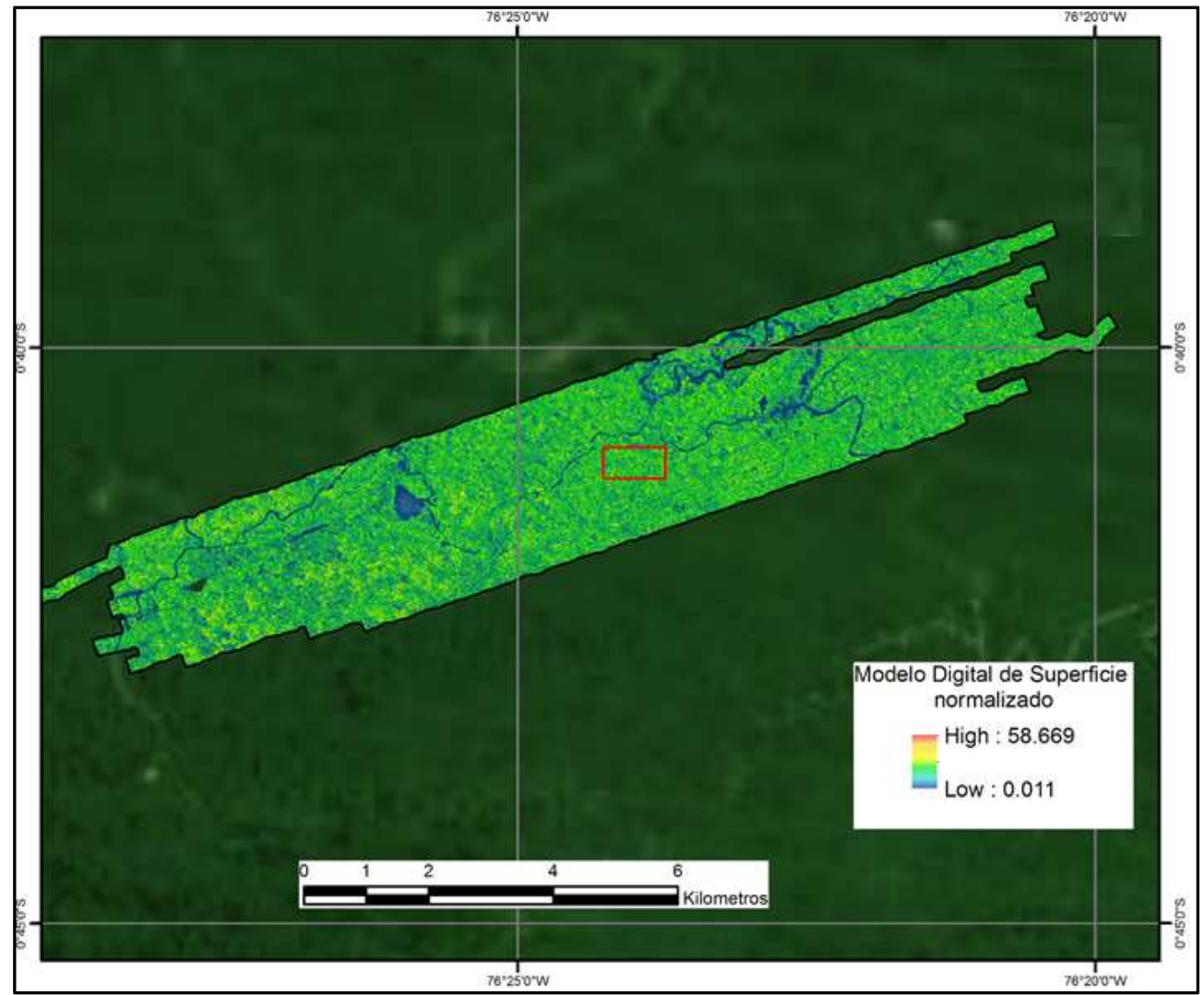

Figura 3.16: Modelo Digital de Superficie normalizado (nMDS) de toda la trayectoria de vuelo LiDAR.

La figura 3.17 muestra el nMDS-3D de la parcela de estudio utilizada para calibrar y validar el modelo general de ACD. En esta figura de puede apreciar a primera vista como se distribuye la vegetación de mayor altura en las partes altas de las colinas (cimas), mientras que la vegetación de menor altura se localiza en las laderas y los valles. En el anexo C se presentan los nMDS donde se puede visualizar a detalle la distribución de la vegetación de cada una de las subparcelas de 1-ha.

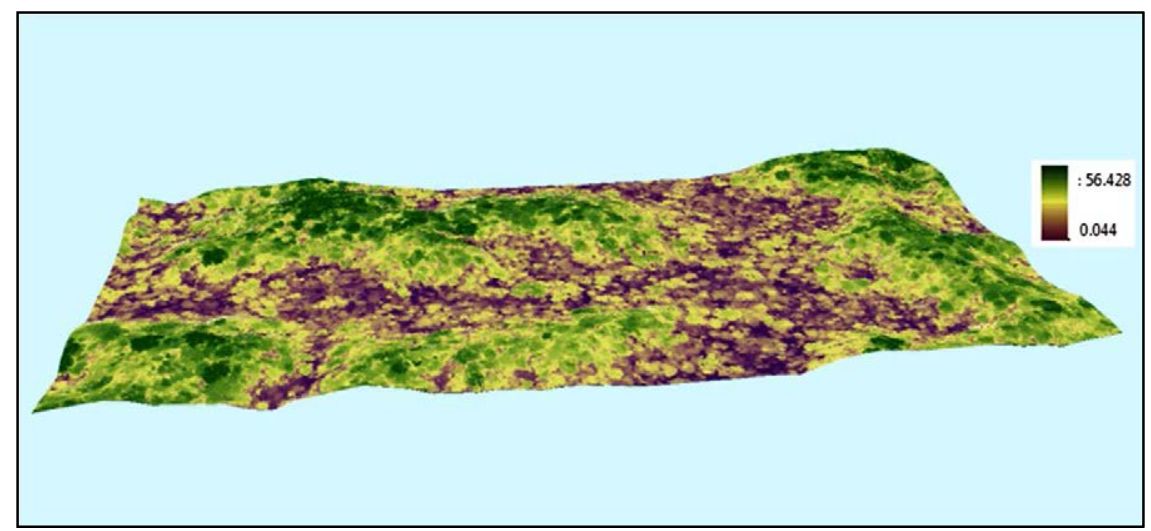

Figura 3.17: Modelo Digital de Superficie normalizado (nMDS-3D) de la parcela de estudio. 


\subsubsection{Cálculo de la altura media al tope del dosel (TCH) y altura media del perfil de dosel (MCH)}

En la primera propuesta del modelo general de estimación de ACD para bosque tropical Asner et al. (2012a), utilizaron la métrica MCH (Mean Canopy Height) que representa la altura media del perfil de dosel. En una publicación posterior, el mismo autor (Asner et al., 2014), sostiene que se debe utilizar la altura media al tope del dosel (TCH) obtenida a partir del modelo Digital de Superficie normalizado (nMDS), en lugar de la altura media del perfil de dosel $(\mathrm{MCH})$ extraídas del perfil vertical de vegetación. Esto se debe a que diferentes tipos de sensores LiDAR producen valores inconsistentes de altura media del perfil del dosel a causa de las características concretas y de las especificaciones de los instrumentos que se utilizan. En particular, por la potencia y divergencia del haz de láser, razón por la cual la métrica TCH es más consistente.

El grado de penetración del haz del láser está determinado por una interacción compleja entre la señal láser, las características de la vegetación y la sensibilidad del receptor LiDAR (Chasmer et al., 2006; Hopkinson, 2007), lo que provoca diferencias entre las mediciones LiDAR de la distribución vertical de perfil de dosel sobre las que se calculan el MCH y otras métricas similares (Næsset, 2009). Este problema afecta a todas las métricas LiDAR que son sensibles al perfil vertical de la vegetación, como es el caso del percentil 50, percentil 80, y de muchas otras métricas (Ni-Meister et al., 2010). Las métricas obtenidas del nMDS, a partir de los primeros retornos, no son insensibles a las variaciones en los parámetros de adquisición, sin embargo, pueden ser más estables que las métricas extraídas del perfil vertical de vegetación (Vincent et al., 2012). A modo de ejemplo, se presenta la figura 3.18 que representa el perfil vertical de vegetación de las parcelas $\mathrm{N}^{\mathrm{o}} 31$ y $\mathrm{N}^{\mathrm{o}} 36$.

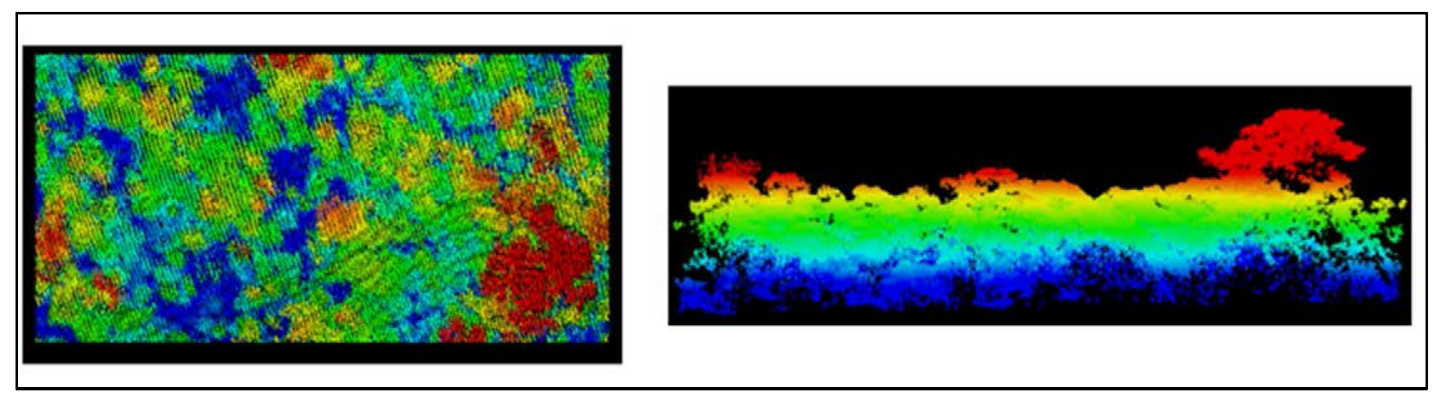

Figura 3.18: Vista superior de la nube de puntos LiDAR de las parcelas $\mathrm{N}^{\mathrm{o}} 31$ y $\mathrm{N}^{\mathrm{o}} 36$ (izquierda); y el perfil vertical de vegetación (derecha). 
Para el cálculo de la altura media al tope del dosel (TCH) se utilizó la herramienta de estadísticas zonales del programa $\operatorname{ArcGIS}^{\circledR}$ 10.1. Esta herramienta resume las estadísticas descriptivas del nMDS (mínimo, máximo, rango, media y desviación estándar) dentro de las zonas de otro conjunto de datos, en este caso, en la malla regular de subparcelas de 1-ha (fishnet).

Para el cálculo de la altura media del perfil de dosel se utilizó la aplicación cloudmetrics en el programa FUSION (McGaughey, 2013). Este programa calcula una cantidad de parámetros estadísticos que describen el conjunto de datos LiDAR. Las métricas o estadísticas son calculadas utilizando todos los puntos de elevación disponibles (todos los retornos). En el anexo D se presenta el resumen de las métricas TCH y $\mathrm{MCH}$ con sus medidas de variación, para cada una de las subparcelas. La figura 3.19 presenta el análisis gráfico del coeficiente de variación (\%) de la elevación de estas 2 métricas calculadas en cada una de las subparcelas.

Como se puede observar, la altura media al tope del dosel (TCH) presenta menor variabilidad que la altura media del perfil de vegetación $(\mathrm{MCH})$, lo que corrobora el uso de la métrica TCH en lugar de la métrica $\mathrm{MCH}$.

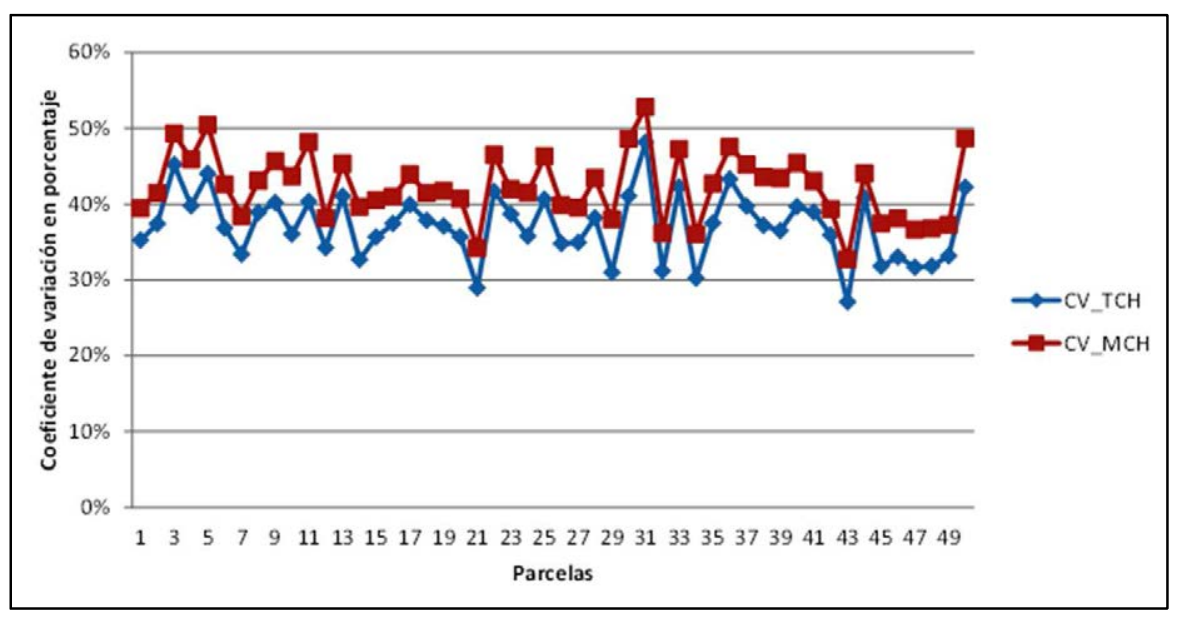

Figura 3.19: Comparación de los coeficientes de variación del TCH y MCH para cada una de las subparcelas.

\subsection{Diseño de muestreo}

El éxito del diseño de muestreo consiste en seleccionar las estrategias de muestreo más adecuadas a la luz de un conjunto de objetivos y limitaciones generales. El diseño de muestreo se puede dividir en dos grupos principales (Köhl et al., 2006): 
1. Diseño de muestreo sin información auxiliar.

2. Diseño de muestreo con información auxiliar.

Las poblaciones que se estudian en el inventario forestal pueden ser descritas por una larga lista de atributos, algunos pueden estar asociados con los árboles, y otros con el entorno en el que crecen. La información sobre estos atributos puede estar disponible o no para los analistas en el momento en el que se planifica el inventario. En los diseños de muestreo sin información auxiliar, se utilizan sólo las observaciones de las variables de interés para derivar los parámetros. En el diseño de muestreo con información auxiliar, en cambio, la información disponible que de alguna manera está asociada con el atributo de interés, se incorpora en el diseño para la estratificación, o en los estimadores en forma de predictor. En esta tesis para proponer el diseño de muestreo estratificado se utilizó información auxiliar obtenida a partir de los datos LiDAR.

\subsubsection{Muestreo estratificado}

En el muestreo estratificado utilizamos información auxiliar para estratificar toda la población. La estratificación tiene como objeto la formación de grupos de elementos con valores de atributos más o menos similares. La estratificación perfecta elimina la variación dentro del estrato, lo que es casi imposible en la práctica. En el caso ideal, una sola muestra de cada estrato sería suficiente para tener un conocimiento completo sobre el parámetro de población de interés, ya que no habría varianza dentro del estrato, y todos los elementos tendrían el mismo valor del atributo. A medida que nos alejamos del caso ideal, se necesitan más muestras para estimar con precisión la media de un estrato. En otras palabras, la estratificación tiene por objeto dividir una población en un número de partes que sean lo más homogéneas posibles.

\subsubsection{Hábitats topográficos en la zona de estudio}

El Parque Nacional Yasuní es una zona protegida donde la influencia humana es actualmente escasa, por lo que no existe cambio de uso del suelo, con excepción de pequeñas áreas donde existe una infraestructura que fue construida para la extracción de petróleo. La mayor parte del Parque está cubierto por bosques maduros intactos. En un estudio realizado por Valencia et al. (2004b), en la parcela de 50 hectáreas se identificaron 2 
hábitats topográficos bien definidos, el valle y la colina (figura 3.20).

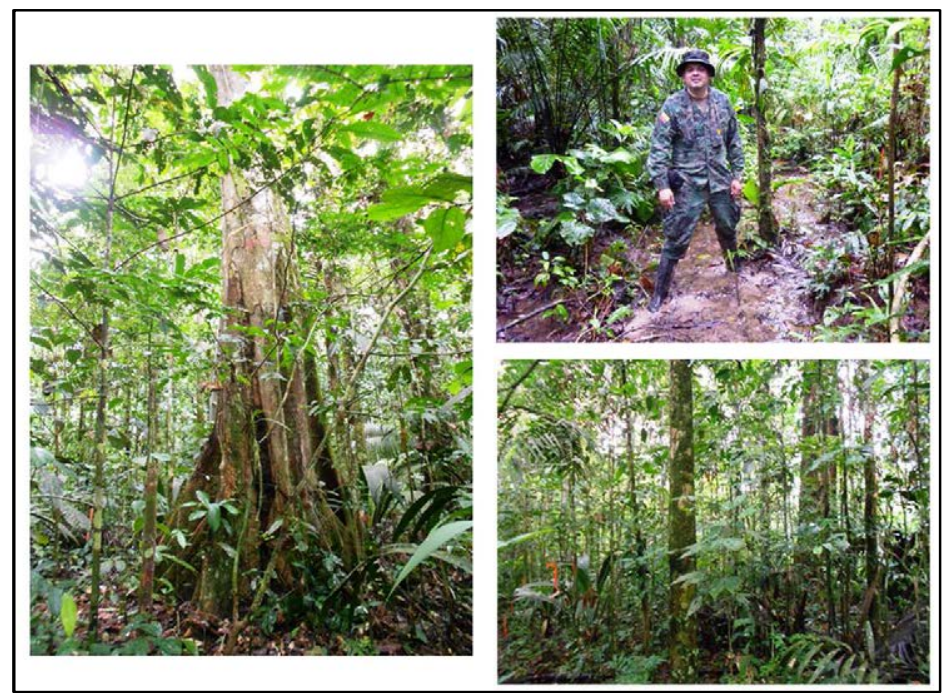

Figura 3.20: Hábitats topográficos en la zona de estudio: colina (izquierda); valle inundado (superiorderecha); valle (inferior-derecha).

En un estudio sobre biomasa en la misma parcela, Valencia et al. (2009) encontraron que la biomasa es más baja en el valle que en la colina. Las diferencias significativas encontradas sugieren que los bosques de tierra firme son un mosaico de hábitats naturales, y que esta variación de hábitats es, en parte, responsable de la variación en las existencias de biomasa que se observa en los bosques amazónicos. Es importante señalar que la mayoría de las especies de árboles en la zona de estudio, se distribuyen de forma no aleatoria a través de gradientes ambientales de luz, agua y nutrientes (Romero-Saltos et al., 2001; Valencia et al., 2004a). A fin de tener una perspectiva rápida sobre la topografía y la estructura vertical de la vegetación, en la figura 3.21 se muestra el perfil longitudinal y transversal del MDT y la nube de puntos LiDAR con color RGB transferidos de la ortofotografía.

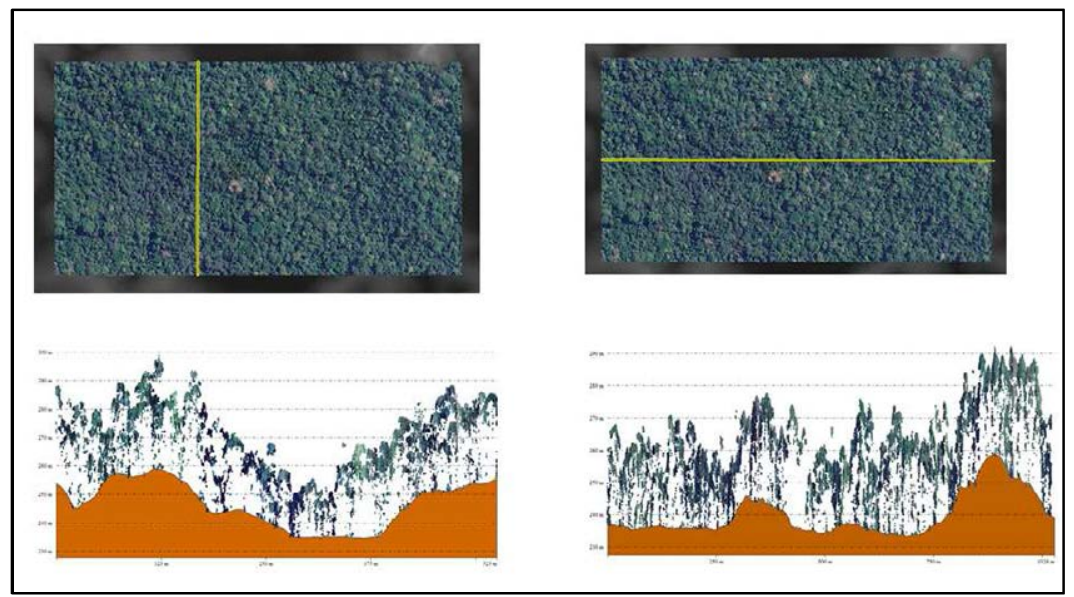

Figura 3.21: Perfil longitudinal y transversal de la parcela de estudio con la nube de puntos LiDAR 
visualizada con color RGB transferidos de la ortofotografía.

\subsubsection{Determinación de posiciones topográficas mediante análisis geo- estadístico}

En la región comprendida entre los Andes y la llanura amazónica, la composición florística, la química de la cubierta forestal, la estructura y la función de los bosques, están muy inter-correlacionados a través de gradientes de elevación y de fertilidad del suelo (Asner et al., 2014b). En un estudio reciente de Réjou-Méchain et al. (2014) sobre las consecuencias de la estructura espacial de la biomasa forestal en el cartografiado del ACD en bosques tropicales, se destaca la importancia de la topografía como un factor que debe ser tomado en cuenta en el diseño de muestreo en los inventarios forestales, y en las calibraciones de los modelos de estimación de variables forestales.

\subsubsection{Estratificación por posiciones topográficas}

Los productos derivados de datos LiDAR permiten caracterizar eficientemente la estructura del terreno y la vegetación. Sin embargo, pocas veces se han utilizado para la estratificación en esquemas de calibración de modelos de ACD. En base a los hallazgos mencionados anteriormente, se consideró necesario estratificar la parcela de estudio por posiciones topográficas (valle, ladera y cima).

Para la estratificación se utilizó la variable geomorfométrica (elevación) y la variable hidrológica (índice topográfico de humedad) derivadas del MDT obtenido con LiDAR, sacando así ventaja de la precisión y resolución espacial obtenida en este producto. Un aspecto clave consistió en determinar qué variable se correlacionaba de mejor manera con las densidades de carbono en la zona de estudio. En un estudio realizado en bosque tropical en Panamá, Mascaro et al. (2011b) encontraron que la pendiente es el parámetro más importante que controla la densidad de carbono sobre el suelo. Moore et al. (1993) demostraron que el índice topográfico de humedad (TWI o Topographic Wetness Index), está correlacionado con varios atributos del suelo tales como el porcentaje de limo, el contenido de materia orgánica y de fósforo. De la misma forma, Kanagaraj et al. (2011) encontraron que el TWI, la pendiente y la elevación, han sido los principales determinantes de la asociación de especies en árboles tropicales. 


\subsection{5. Índice topográfico de humedad (TWI)}

Esta variable es un atributo compuesto del terreno, que proviene de dos variables primarias: el área de acumulación de flujo y la pendiente. Se utiliza principalmente para identificar vías de flujo hidrológicos, y es muy útil para caracterizar procesos biológicos, para identificar patrones de vegetación y calidad del sitio, así como para predecir el contenido de humedad del suelo e identificar áreas de erosión y /o deposición potencial de materiales (Martinez-Casasnovas, 1999).

El TWI es una variable que define la tendencia de una celda a acumular agua (Gruber \& Peckham, 2009). Está relacionada con la humedad del suelo y refleja la tendencia de éste a generar escorrentía; relaciona la dirección y acumulación de flujo, representadas por el área de captación o área de acumulación con la pendiente del terreno. Se calcula con la ecuación 3.4 (Beven \& Kirkby, 1979):

$$
T W I_{i}=\ln \left[\frac{A s_{i}}{\operatorname{Tan} \beta_{i}}\right]
$$

Donde $\mathrm{As}_{\mathrm{i}}=$ Área de acumulación de flujo en la celda $\mathrm{i}$

$\mathrm{B}_{\mathrm{i}}=$ pendiente local de la celda $\mathrm{i}$

El área de acumulación de flujo, área aportante o área de captación es la superficie que situada aguas arriba de un punto de interés, recoge el flujo que recibe y lo transita por dicho punto. Es una superficie proyectada en un plano horizontal. En un mapa raster se obtiene sumando todas las celdas que contribuyen con flujo a la celda de interés, situada aguas abajo y multiplicando por la superficie de cada celda. Cuando el área de captación se incrementa y el gradiente decrece, el índice de humedad y el contenido de humedad del suelo decrecen. Al combinar datos sobre gradiente y área de captación, dicho índice toma en cuenta tanto la geometría de la pendiente local como la localización del sitio en el paisaje. Por lo tanto, correlaciona mejor con la humedad del suelo que con el área específica (Leiva Gutiérrez, 2012). Valores altos del índice de humedad indican potencial para la acumulación de agua en el suelo, y coincide con aquellas zonas de baja pendiente y con un valor alto de área de drenaje específica. Valores bajos del índice de humedad indican bajo potencial topográfico para la acumulación de agua en el suelo, ya sea por tratarse de un área con una cuenca de captación pequeña o por un valor alto de la pendiente, indicador de suelos bien drenados. 
En esta investigación, el índice topográfico de humedad (figura 3.22) se calculó con la herramienta Geomorphometric and Gradient Metrics (Evans et al., 2014) en el programa ArcGIS $^{\circledR} 10.1$, a partir del Modelo Digital del terreno generado con datos LiDAR.

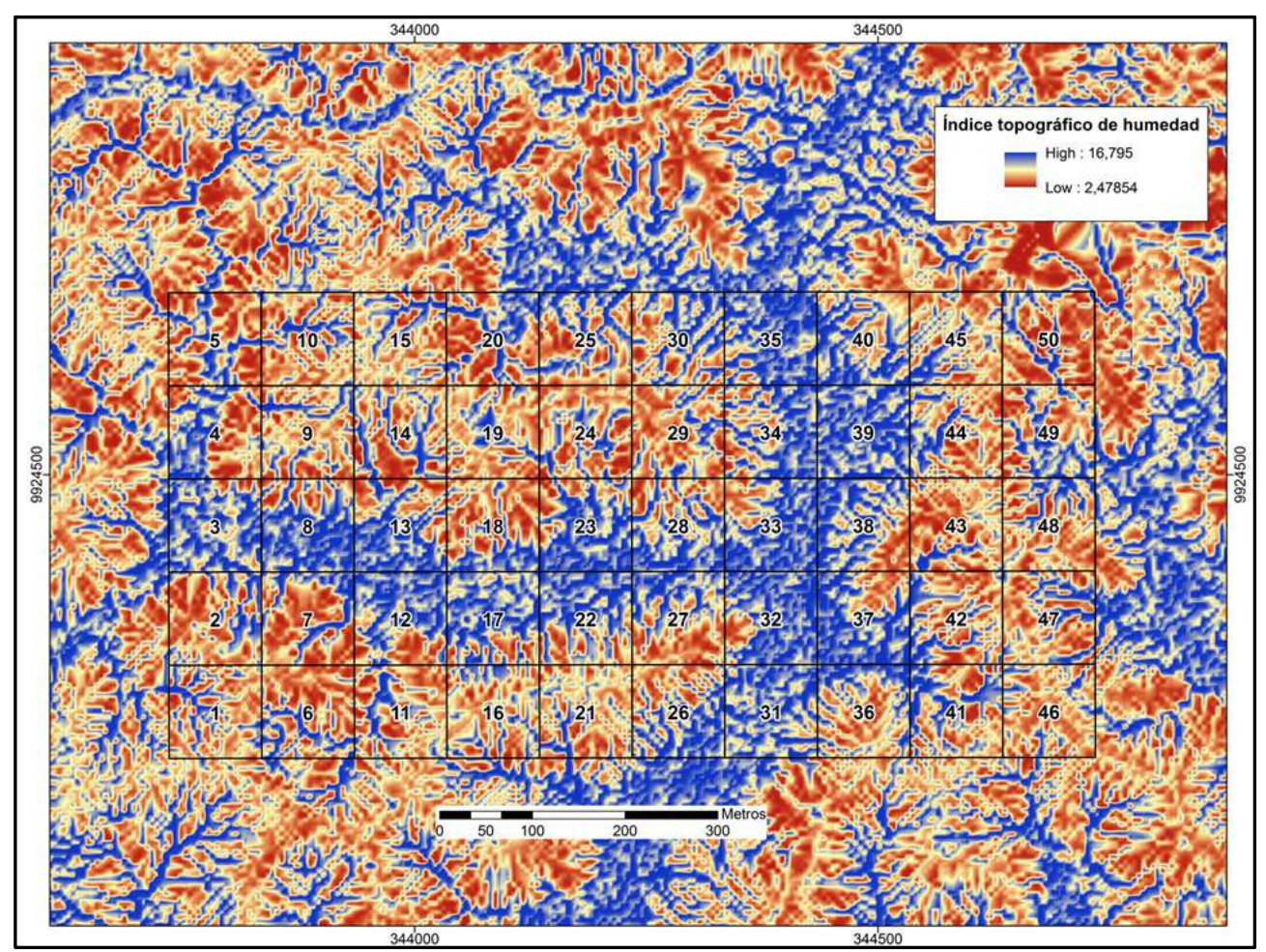

Figura 3.22: Índice Topográfico de humedad de la zona de estudio.

A continuación se calcularon las estadísticas de elevación del MDT y del TWI para cada una de las subparcelas (min, max, rango, media, desviación estándar), utilizando la herramienta de estadísticas zonales en $\operatorname{ArcGIS}^{\circledR}$ 10.1. Estas estadísticas fueron utilizadas para la selección de las posiciones topográficas de las subparcelas. En las figuras 3.23 y 3,24 se muestran los valores de la elevación media y el TWI medio para cada subparcela.

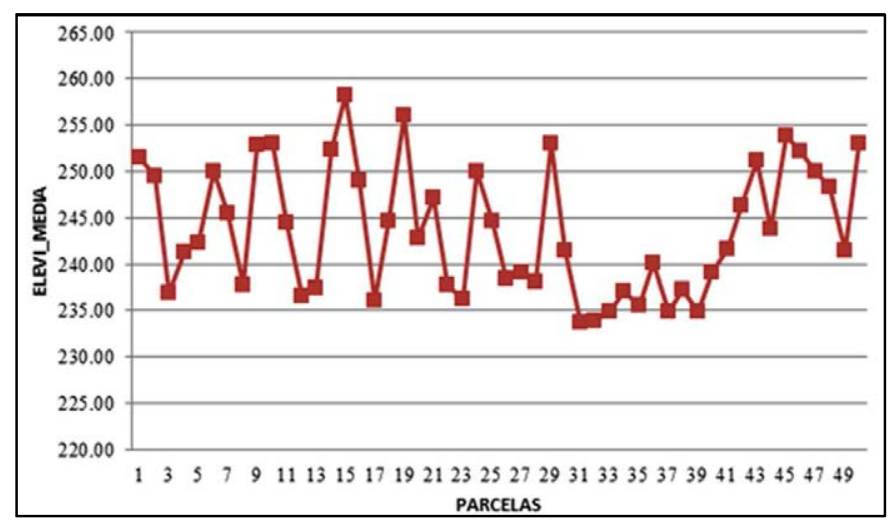

Figura 3.23: Elevación media por subparcelas. 


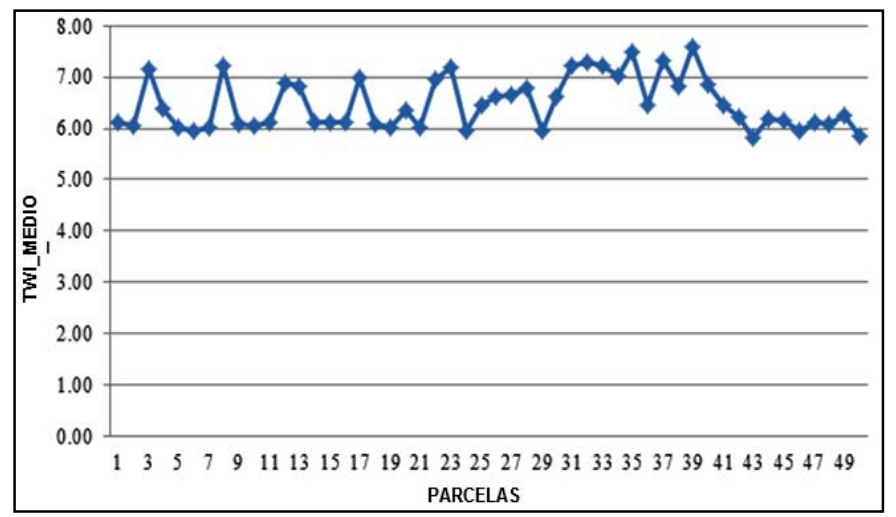

Figura 3.24: Índice topográfico de humedad por subparcela.

Se calcularon los coeficientes de correlación de Pearson entre la biomasa sobre el suelo y el índice de humedad topográfica media a nivel de subparcela $(\rho=-0.59)$, y el coeficiente de correlación de Pearson entre la biomasa sobre el suelo y la elevación media $(\rho=0.68)$. A continuación se realizó la estratificación de la muestra por posiciones topográficas (valle, ladera y cima).

Las 3 posiciones topográficas fueron definidas después de realizar el análisis de las estadísticas zonales medias de elevación y el índice topográfico de humedad. Las subparcelas con elevación media $<239.10$ m y TWI media $>6.60$ fueron clasificadas como valle, las subparcelas con elevación media $>248.20$ y TWI media $<6.12$ fueron clasificadas como cima, y las subparcelas restantes fueron clasificadas como ladera (Tabla 3.7).

Tabla 3.7: Resumen de las variables del modelo por posiciones topográficas.

\begin{tabular}{|c|c|c|c|c|c|}
\hline \multirow{3}{*}{$\begin{array}{l}\text { Posición } \\
\text { topográfica }\end{array}$} & \multirow{3}{*}{$\begin{array}{c}\text { Número } \\
\text { de parcelas }\end{array}$} & \multicolumn{4}{|c|}{ Media (DE) } \\
\hline & & LiDAR TCH & Área basal & Densidad de madera & $\mathbf{A G B}_{\text {campo }}$ \\
\hline & & (m) & $\left(\mathrm{m}^{2} h \mathrm{a}^{-1}\right)$ & $\left(\mathrm{g} \mathrm{cm}^{-3}\right)$ & $\left(\mathrm{Mg} \mathrm{ha}^{-1}\right)$ \\
\hline Valle & 18 & $20.60(1.8)$ & $24.37(2.5)$ & $0.557(0.01)$ & $198.24(29.5)$ \\
\hline Cima & 16 & $23.50(1.1)$ & $29.90(2.6)$ & $0.574(0.01)$ & $265.39(33.3)$ \\
\hline ladera & 15 & $21.47(1.9)$ & $27.41(3.1)$ & $0.563(0.01)$ & $228.30(37.06)$ \\
\hline $\mathrm{C}_{\mathrm{FS}}$ & 1 & 22.74 & 30.76 & 0.471 & 199.40 \\
\hline
\end{tabular}

$\mathrm{C}_{\mathrm{FS}}=$ Cima con remanente de bosque secundario; $\mathrm{TCH}=$ altura media al tope del dosel; $\mathrm{DE}=$ desviación estándar. 


\subsection{Análisis de la distribución de AGB en la parcela de estudio}

Para el análisis de la variabilidad local de la biomasa sobre el suelo se elaboró una base de datos geográfica en ArcGIS ${ }^{\circledR}$ 10.1. La base de datos geográfica contiene información sobre: a) identificación del árbol, b) ubicación espacial, c) código de especie, d) diámetro del árbol, e) área basal, f) valor de biomasa sobre el suelo, y g) valor de densidad de madera de cada uno de los individuos con DAP $\geq 1 \mathrm{~cm}$ en las 50 subparcelas de estudio (figura $3.25)$.

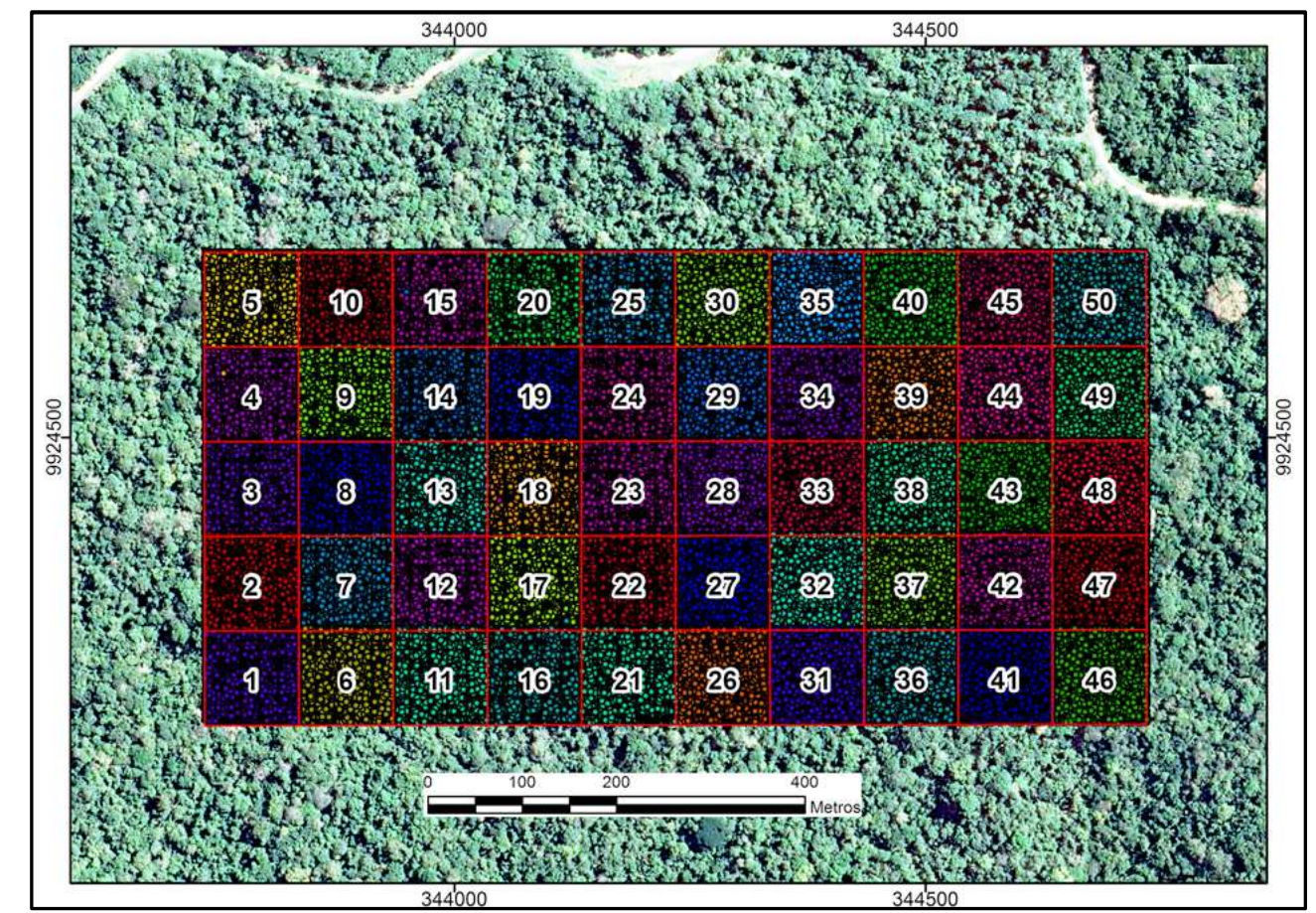

Figura 3.25: Captura de pantalla de los feature-class de la base de datos geográfica, cada color representa una subparcela de 1-ha.

\subsubsection{Análisis de Punto Caliente (hotspot)}

Para localizar los patrones de distribución de la biomasa en la parcela, se utilizaron las herramientas de estadística espacial en el programa $\operatorname{ArcGIS}^{\circledR}$ 10.1. La herramienta análisis de punto caliente o hotspot calcula la estadística Gi* de Getis-Ord (Getis \& Ord, 1992) para cada entidad en un conjunto de datos. Esta herramienta identifica clusters o agrupaciones espaciales estadísticamente significativas de valores altos (puntos calientes) y valores bajos (puntos fríos) que proporcionan indicios importantes sobre los procesos espaciales subyacentes que producen patrones específicos. La estadística Gi* devuelta para cada 
entidad es una puntuación z.

Esta herramienta funciona mediante la búsqueda de cada entidad dentro del contexto de entidades vecinas. Una entidad con un valor alto es interesante, pero es posible que no corresponda a un punto caliente estadísticamente significativo. Para que sea un punto caliente estadísticamente significativo, una entidad debe tener un valor alto y estar rodeada por otras entidades con valores altos. La suma local para una entidad y sus vecinos se compara proporcionalmente con la suma de todas las entidades; cuando la suma local es muy diferente de la esperada, y esa diferencia es demasiado grande como para ser el resultado de una opción aleatoria, se obtiene una puntuación z estadísticamente significativa. Para las puntuaciones $\mathrm{z}$ positivas que son estadísticamente significativas, mientras más grande sea la puntuación z, más intensa será la agrupación espacial o clustering de valores altos. Para las puntuaciones $\mathrm{z}$ negativa que son estadísticamente significativas, mientras más pequeña sea la puntuación z, más intenso será el clustering de valores bajos. Una puntuación z cercana a cero significa que no hay agrupación espacial.

\subsubsection{Consideraciones del análisis de punto caliente (hotspot)}

Se deben considerar 3 factores al momento de realizar un análisis de punto caliente:

1. Identificación del campo de análisis o campo de entrada. En este caso, el campo de entrada es el valor de AGB de cada árbol en la parcela de 50-ha.

2. Conceptualización de la relación espacial. Se utilizó la zona de indiferencia (Combinación de distancia inversa y banda de distancia fija).

3. Selección de una distancia de umbral adecuada. Se seleccionó un umbral de 100 metros considerando el tamaño de la subparcela de análisis (100 m x 100 m).

Para el análisis, se utilizaron las estimaciones de AGB de cada árbol con DAP $\geq 10 \mathrm{~cm}$ en la parcela (34.567 individuos). El resultado del análisis hotspot fue el mapa con la distribución de la AGB en la parcela que se presenta en la figura 3.26. 


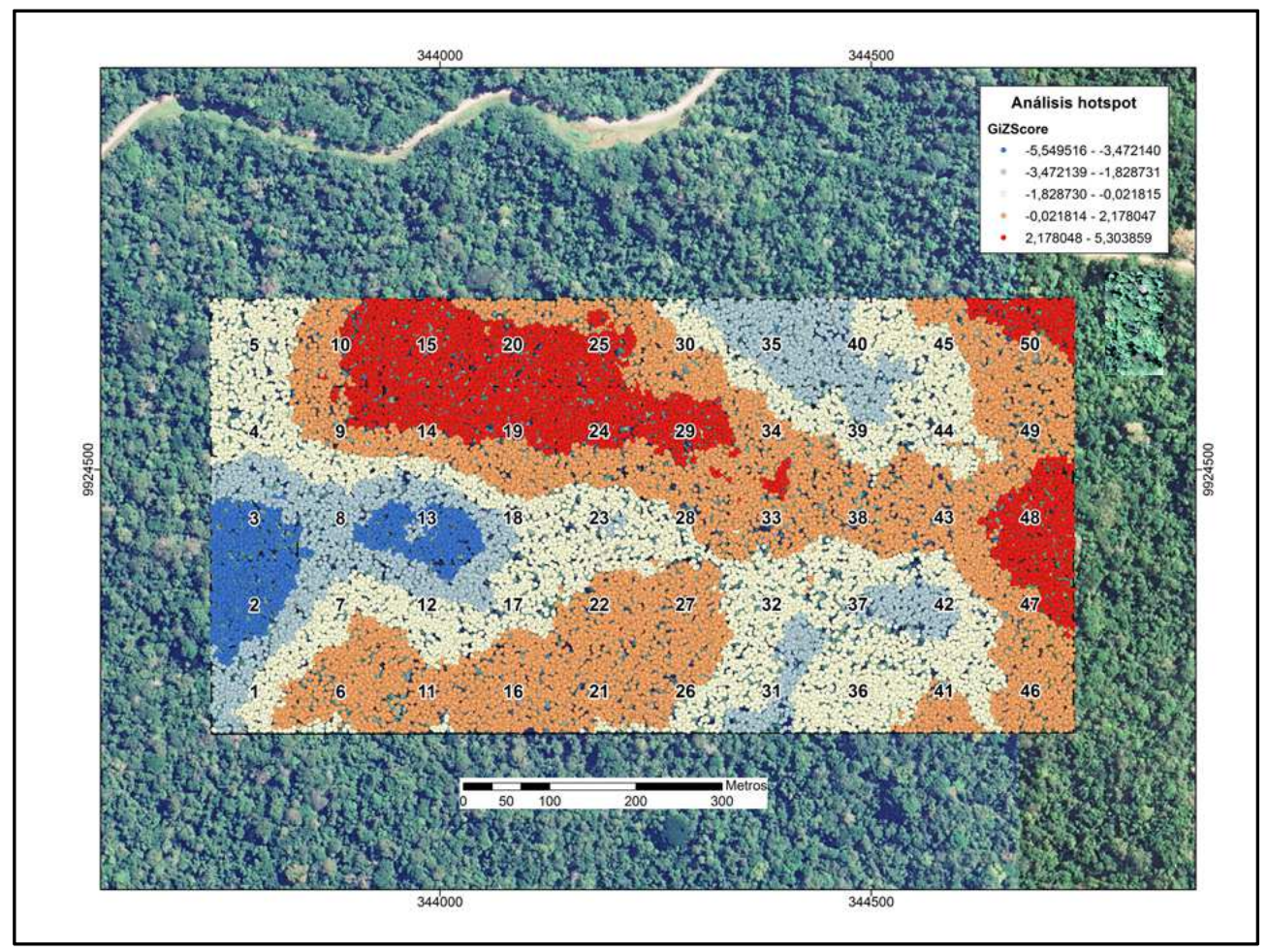

Figura 3.26: Análisis de punto caliente en la parcela de 50-ha. Las agrupaciones de puntos en color rojo representan puntos calientes de alta densidad de biomasa, mientras que las agrupaciones en color azul representan puntos fríos de baja densidad de biomasa.

Los resultados muestran que la mayor concentración de biomasa (hotspot) se localiza en las partes altas (cimas), mientras que las menores densidades de biomasa (coldspot) se encontraron en el valle. Los lugares con menor concentración de biomasa corresponden a la parcela $\mathrm{N}^{\circ} 2$, que contiene el remanente de bosque secundario $(199.4 \mathrm{Mg} / \mathrm{ha})$, la parcela $\mathrm{N}^{\circ}$ 13 que contiene los árboles más pequeños del área de estudio $(\mathrm{TCH}$ media= $16.9 \mathrm{~m}$.), y las parcelas $\mathrm{N}^{\circ} 3,35,37$ y 39 que se encuentran en el valle y presentan condiciones hidrológicas como la anoxia (condiciones tipo pantano), que son fácilmente identificadas a través del índice TWI.

\subsubsection{Cálculo de la Autocorrelación espacial en la parcela}

Un tema conceptual importante en el análisis de los datos espaciales es la presencia de autocorrelación espacial. La autocorrelación espacial es esencialmente la naturaleza de la geografía, y, en consecuencia, estará casi siempre presente en los datos espaciales. La autocorrelación espacial es la expresión formal de la primera ley de la Geografía (Tobler, 1970): “Todas las cosas están relacionadas entre sí, pero las cosas más próximas en el espacio tienen una relación mayor que las distantes”. Griffith (1987) realizó un análisis de 
los impactos de la autocorrelación espacial en la estadística inferencial, en el que concluye que, en general, no afecta a la exactitud de la estimación de los parámetros. Sin embargo, puede ser la causa para llegar a conclusiones incorrectas respecto a si la relación entre las variables o factores son verdaderas o no. En un estudio reciente en bosque tropical, RéjouMéchain et al., (2014) encontraron que la AGB presenta autocorrelación espacial débil a distancias que van desde $20 \mathrm{~m}$. hasta $400 \mathrm{~m}$, y autocorrelación alta en sitios con alta variabilidad topográfica; por lo que al usar parcelas de $100 \times 100 \mathrm{~m}$, como unidades de análisis, se eliminaría al menos parte del problema de la autocorrelación espacial.

La herramienta autocorrelación espacial incremental en $\operatorname{ArcGIS}^{\circledR}$ 10.1, ejecuta la herramienta de autocorrelación espacial (índice I de Moran global) para una serie de distancias en aumento, midiendo la intensidad de la agrupación espacial o clustering para cada distancia. El índice I de Moran global mide la autocorrelación espacial basada en las ubicaciones y los valores de las entidades simultáneamente, es decir de la cantidad de AGB de cada árbol y su posición espacial. La distancia seleccionada como umbral para el análisis de autocorrelación espacial incremental fue de $100 \mathrm{~m}$. (tamaño de la parcela de análisis). El pico mínimo se encuentra en 300 metros, valor que representa la distancia a la que los procesos que promueven el clustering son menos pronunciados. La figura 3.27 muestra las puntuaciones $\mathrm{z}$ asociadas con distancias de 100 metros.

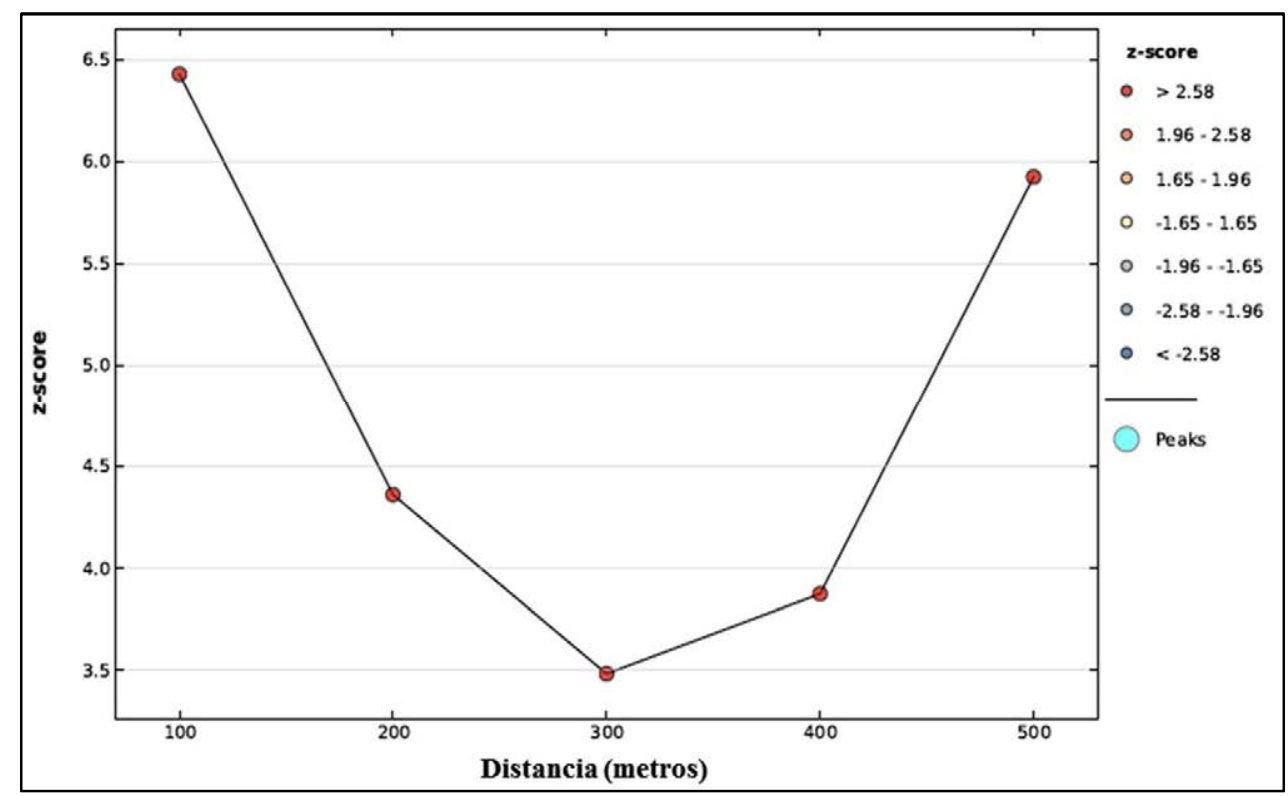

Figura 3.27: Autocorrelación espacial incremental cada 100 m. en la parcela de estudio. 
La tabla 3.8 presenta los resultados del cálculo del índice de Moran, el índice esperado, la varianza, la puntuación z y el p-valor para cada una de las distancias de análisis.

Tabla 3.8: Resumen de la autocorrelación espacial incremental de la AGB en la parcela de 50-ha.

\begin{tabular}{|c|c|c|c|c|}
\hline Distancia (m.) & $\begin{array}{c}\text { Índice de } \\
\text { Moran }\end{array}$ & Varianza & Valor-z & p-valor \\
\hline 100 & 0.009126 & 0.000002 & 6.428136 & 0.000000 \\
\hline 200 & 0.002961 & 0.000001 & 4.358661 & 0.000013 \\
\hline 300 & 0.001388 & 0.000000 & 3.479772 & 0.000512 \\
\hline 400 & 0.001006 & 0.000000 & 3.871819 & 0.000108 \\
\hline 500 & 0.001117 & 0.000000 & 5.923624 & 0.000000 \\
\hline
\end{tabular}

La herramienta Autocorrelación espacial (I de Moran Global) es una estadística deductiva, lo que significa que los resultados del análisis siempre se interpretan dentro del contexto de la hipótesis nula:

$\mathrm{Ho}=$ aleatoriedad espacial completa de la AGB.

$\mathrm{H}_{1}=$ no aleatoriedad espacial completa de la AGB.

Cuando el valor $\mathrm{p}$ es muy pequeño, significa que es muy poco probable (pequeña probabilidad) que el patrón espacial observado sea el resultado de procesos aleatorios, y que por lo tanto, se puede rechazar la hipótesis nula.

\subsection{Modelo de regresión lineal para la estimación de la ACD}

Los enfoques típicos para predecir AGB con datos LIDAR se basan en modelos de regresión lineal que relacionan las métricas LiDAR con estimaciones de biomasa de parcelas de inventario forestal (Säynäjoki et al., 2008). Las métricas derivadas de LiDAR más frecuentemente empleadas para predecir la biomasa incluyen la media o la altura máxima del dosel (Baccini et al., 2013; Clark et al., 2011); y las medidas del perfil vertical de dosel, tales como los percentiles y los cuantiles (Lim \& Treitz, 2004). Estos modelos se obtienen a partir de un estudio estadístico que garantiza su consistencia y rigor matemático, así como su capacidad predictiva. Los pasos básicos para la determinación de modelos de regresión lineal múltiple son (Peña Sánchez de Rivera, 2002): 
1. Análisis preliminar/análisis exploratorio de datos.

2. Determinación y ajuste del modelo.

3. Bondad de ajuste.

4. Diagnosis del modelo.

\subsubsection{Análisis exploratorio de los datos LiDAR}

La primera fase de cualquier análisis de datos implica un proceso de "comprensión de los datos", a través del análisis exploratorio de datos (AED). La comprobación de la integridad de los datos y el estudio de las distribuciones, correlaciones y relaciones entre variables potencialmente importantes, es una parte fundamental del proceso previo de análisis para el desarrollo del modelo y las pruebas de hipótesis. Como mínimo, después del AED, se alcanzará el objetivo previsto de comprender los datos antes de emplear métodos de modelado de datos más complejos. Moffiet et al. (2005) utilizaron AED para evaluar el potencial de los tipos de retornos laser y la intensidad de retorno como variables para la clasificación de árboles individuales o masas forestales según la especie. Tomando en consideración las diferencias encontradas en las estimaciones de AGB en campo a nivel de parcela, y la variabilidad en el análisis de distribución de AGB (Hotspot), se realizó un AED detallado a nivel de subparcelas.

El AED es más efectivo cuando se combinan diferentes programas que permitan visualizar los datos, realizar análisis gráficos exploratorios y calcular parámetros estadísticos que describan el conjunto de datos. Para el análisis exploratorio de los datos LiDAR se utilizaron LASmetrics (USDA-RMRS, Silva et., 2015), que es una aplicación web gratuita para el procesamiento y visualización de datos LiDAR; y cloudmetrics en FUSION (McGaughey, 2013), que calcula una serie de parámetros estadísticos del conjunto de datos LiDAR. Con estos programas se calcularon los principales estadísticos descriptivos, se construyeron las curvas de distribución y diagramas de caja (para visualizar la variabilidad de la altura de la vegetación y ubicar valores extremos); y también los perfiles de la nube de puntos LiDAR en cada una de las subparcelas. Los resultados se presentan en el anexo E.

\subsubsection{Medidas de variabilidad o dispersión}

Las medidas de variabilidad tienen por objeto medir la magnitud de las desviaciones de los valores de la variable con respecto al valor central de la distribución, es decir, definen 
cuan semejante o cuan distinto son cada uno de los valores de la variable con respecto al valor central. La variabilidad es la característica más importante de la distribución, ya que se constituye en un test de validación eficaz, referido al nivel de significación de las medidas de centralización. Las principales medidas de variabilidad son:

\section{Rango.}

2. Rango intercuartílico.

3. Desviación típica o estándar.

4. Coeficiente de variación (CV).

Una de las medidas de variabilidad más representativa es el coeficiente de variación, que hace referencia a la relación entre el tamaño de la media y la variabilidad de la variable analizada. Para caracterizar la heterogeneidad espacial en la parcela, se calcularon los coeficientes de variación en porcentaje (ecuación 3.5) de cada variable forestal estimada en campo y que sería utilizada en el modelado.

$$
C V=\frac{\sigma}{\mu} \times 100
$$

Donde $\sigma$ es la desviación estándar, y $\mu$ es la media aritmética de la variable a una escala espacial de 1-ha.

Un valor alto del CV indica una mayor variabilidad espacial relativa (heterogeneidad) de la densidad de AGB (con respecto a la media). La tabla 3.9 resume las principales medidas de variabilidad de los parámetros estimados en campo para los árboles con DAP $\geq 10 \mathrm{~cm}$.

Tabla 3.9: Coeficiente de variación (CV) de la densidad de AGB, área basal y densidad de madera en la parcela de estudio.

\begin{tabular}{cccc}
\hline & $\begin{array}{c}\text { AGB } \\
\left(\mathbf{M g ~ h a}^{-\mathbf{1}}\right)\end{array}$ & $\begin{array}{c}\text { Área Basal } \\
\left(\mathbf{m ~}^{\mathbf{2}} \mathbf{~ h a}^{\mathbf{- 1}}\right)\end{array}$ & $\begin{array}{c}\text { Densidad de madera } \\
\left(\mathbf{g ~ c m}^{\mathbf{- 3}}\right)\end{array}$ \\
\hline Rango & $140.58-317.11$ & $19.86-33.93$ & $0.472-0.614$ \\
Media & 228.77 & 27.19 & 0.564 \\
Desviación estándar & 42.82 & 3.56 & 0.020 \\
Coeficiente de variación $(\%)$ & 18.72 & 13.09 & 3.546 \\
\hline
\end{tabular}

Los resultados del coeficiente de variación de la estimación de AGB obtenidos, 
utilizando subparcelas de 1-ha, concuerdan con los resultados encontrados por RéjouMéchain et al. (2014) en su estudio. El valor del CV (\%) para 25 ha fue de 18.80, mientras que el CV (\%) para la parcela completa fue de 18.72 .

\subsubsection{Condiciones previas para utilizar regresión lineal múltiple}

Para modelizar las variables mediante regresión lineal múltiple, se deben cumplir los siguientes requisitos:

1. La variable respuesta se distribuye normalmente.

2. La variable respuesta se relaciona linealmente con las variables predictivas.

3. La varianza residual es constante (Homocedasticidad).

Para comprobar gráficamente si las variables que intervienen en el modelo siguen una distribución normal, se obtuvo un histograma de frecuencias (Figuras3.28; 3.29; 3.30). Es recomendable hacer también el test de Shapiro-Wilk (Shapiro y Wilk, 1965) para comprobar de forma cuantitativa.

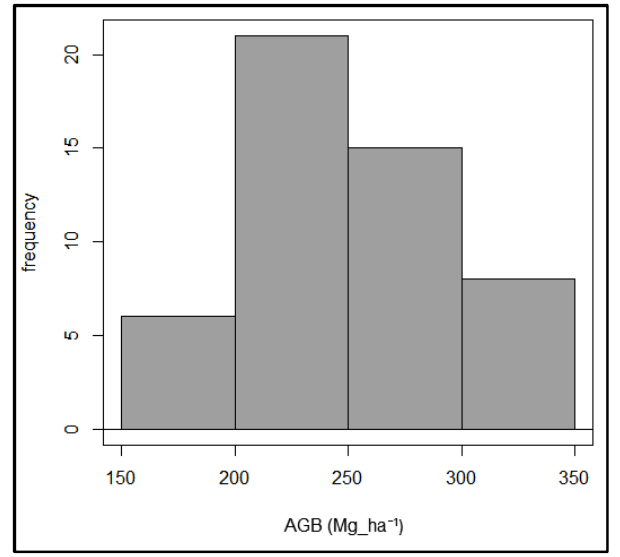

Figura 3.28: Histograma de frecuencias de la AGB en campo.

Test de normalidad de Shapiro-Wilk para la AGB:

$\mathrm{W}=0.9702, \mathrm{p}$-value $=0.2366$ 


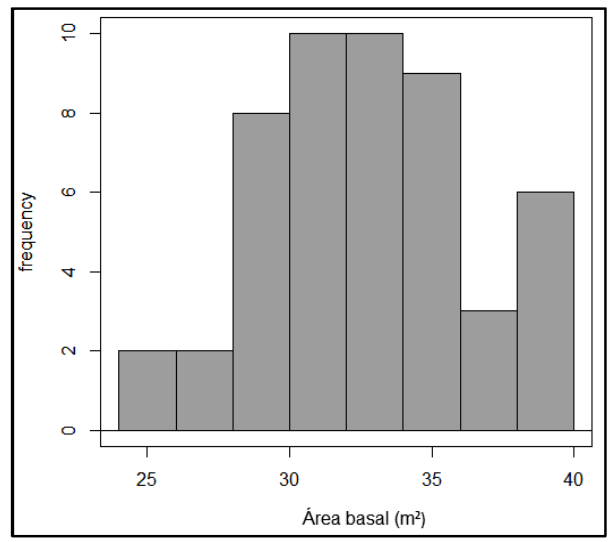

Figura 3.29: Histograma de frecuencias del área basal.

Test de normalidad de Shapiro-Wilk para el área basal:

$\mathrm{W}=0.9729, \mathrm{p}$-value $=0.3037$

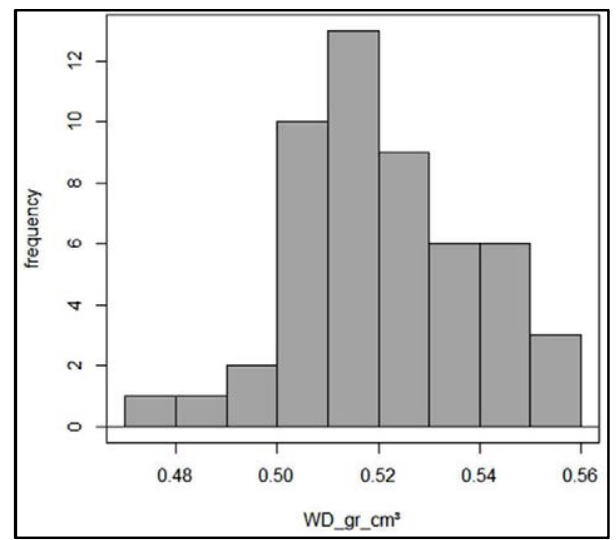

Figura 3.30: Histograma de frecuencias de la densidad de madera.

Test de normalidad de Shapiro-Wilk para la densidad de madera:

$\mathrm{W}=0.9866, \mathrm{p}$-value $=0.8286$

Como el p_valor $>0.05$ para las 3 variables, no se puede rechazar la hipótesis de normalidad. Como conclusión se asume que la distribución de frecuencias de la AGB, el área basal y la densidad de madera son normales.

\subsubsection{Análisis de correlación lineal de los datos}

En probabilidad y estadística, la correlación indica la fuerza y la dirección de una relación o asociación que existe entre dos variables estadísticas. La correlación entre dos variables no implica, por sí misma, ninguna relación de causalidad. La relación entre dos 
variables cuantitativas queda representada mediante la línea de mejor ajuste, trazada a partir de la nube de puntos. Existen diversos coeficientes que miden el grado de correlación, adaptados a la naturaleza de los datos. Uno de ellos es el coeficiente de correlación lineal de Pearson, que se denota con r, y su valor oscila entre -1 y 1.

En esta investigación, nos interesa cuantificar la intensidad de la relación lineal entre la AGB, TCH, MCH, el área basal y la densidad de madera (WD). Este análisis nos permite identificar cuáles son las variables que se relacionan mejor con la variable respuesta que se quiere modelizar, en este caso la AGB. Para realizar los cálculos de los índices de correlación se utilizó el software estadístico R-Commander versión 3.1.2 (Fox, 2005), y los resultados obtenidos se muestran en la tabla 3.10:

Tabla 3.10: Matriz de correlaciones de Pearson de las variables (número de observaciones =50).

\begin{tabular}{cccccc}
\hline & AGB & TCH & MCH & Área basal & WD \\
\hline AGB & 1.000 & 0.766 & 0.761 & 0.953 & 0.558 \\
TCH & 0.766 & 1.000 & 0.987 & 0.761 & 0.273 \\
MCH & 0.761 & 0.987 & 1.000 & 0.741 & 0.301 \\
Área basal & 0.953 & 0.761 & 0.741 & 1.000 & 0.340 \\
WD & 0.558 & 0.273 & 0.301 & 0.340 & 1.000 \\
\hline
\end{tabular}

Las variables estimadas TCH, MCH y el área basal están altamente correlacionados con las mediciones de $\mathrm{AGB}$, mientras que existe una correlacion menor de éstos parámetros con la variable densidad de madera. La diferencia de la correlación entre TCH y la AGB es mínima en comparación con MCH y AGB. En función de la probabilidad fiducial asociada al 95\%, en este trabajo verificamos que el p-valor es inferior a 0.05 (tabla 3.11) por lo que se considera que la correlación es estadísticamente significativa, con excepción del TCH, el $\mathrm{MCH}$ y el área basal con la densidad de madera (WD).

Tabla 3.11: Tabla de probabilidad asociada de las variables del modelo $(n=50)$.

\begin{tabular}{cccccc}
\hline & AGB & TCH & MCH & Área basal & WD \\
\hline AGB & & $<.0001$ & $<.0001$ & $<.0001$ & $<.0001$ \\
TCH & $<.0001$ & & $<.0001$ & $<.0001$ & 0.0544 \\
MCH & $<.0001$ & $<.0001$ & & $<.0001$ & 0.0334 \\
Área basal & $<.0001$ & $<.0001$ & $<.0001$ & & 0.0156 \\
WD & $<.0001$ & 0.0544 & 0.0334 & 0.0156 & \\
\hline
\end{tabular}

Con la matriz de diagramas de dispersión (figura 3.31), se puede visualizar de un solo 
pantallazo la relación que existe entre las variables candidatas del modelo general.

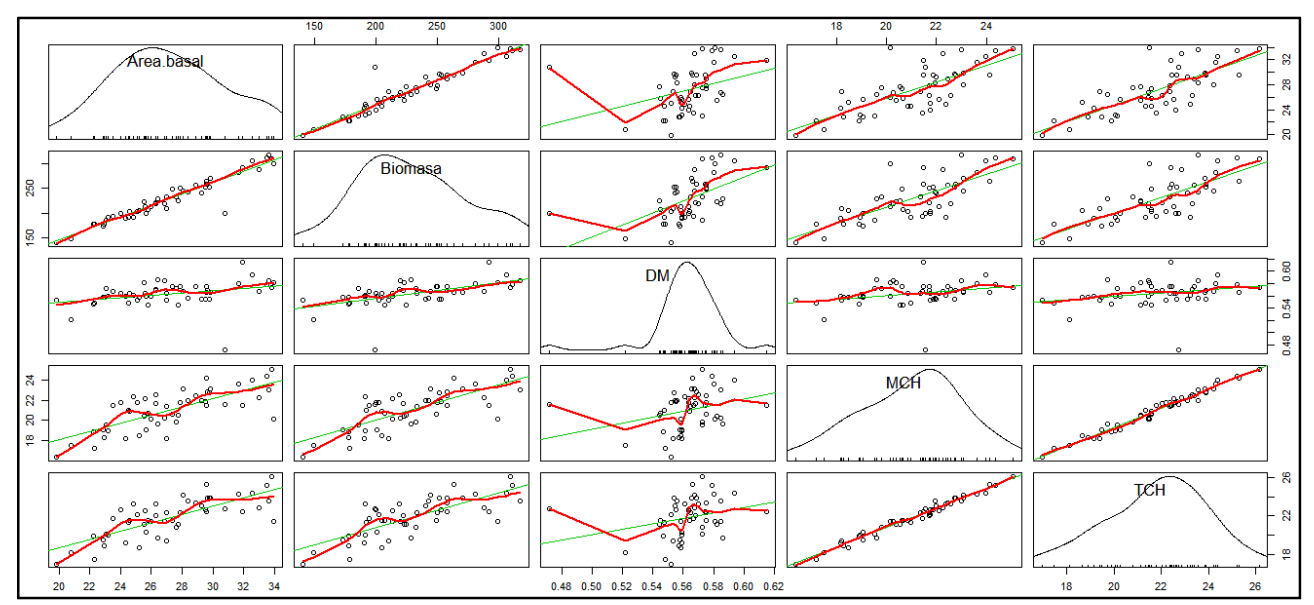

Figura 3.31: Matriz de diagramas de dispersión de las variables candidatas del modelo general.

\subsection{Aplicación del modelo general de estimación de ACD}

Uno de los objetivos generales de la investigación es diseñar un esquema de calibración para el modelo general de estimación de ACD propuesto en la literatura (Asner et al., 2014; Asner et al., 2012). Este modelo ha sido utilizado en varios estudios en bosques tropicales. El esquema de calibración incluye la propuesta de estratificación por posiciones topográficas utilizando productos derivados de LiDAR. Partiendo de esta estratificación, se seleccionaron las subparcelas para el ajuste y validación del modelo general. Con este esquema de calibración propuesto, se busca contribuir a la evaluación de la robustez y precisión del modelo general.

\subsubsection{Selección de las subparcelas para el ajuste y validación del modelo general}

En la selección de las subparcelas para el ajuste y validación del modelo general se consideraron las posiciones topográficas, de tal forma que se asegurará disponer de una distribución que cubriera los 2 hábitats topográficos predominantes en la zona de estudio. Para la selección de la muestra se utilizó el método de fragmentación, es decir el 66 \% de la muestra fue utilizada para el ajuste y el 33\% para la validación. Para el ajuste del modelo se consideraron 32 parcelas, y para la validación 18 parcelas (figura 3.32), que fueron seleccionadas usando muestreo aleatorio simple dentro de cada posición topográfica. 


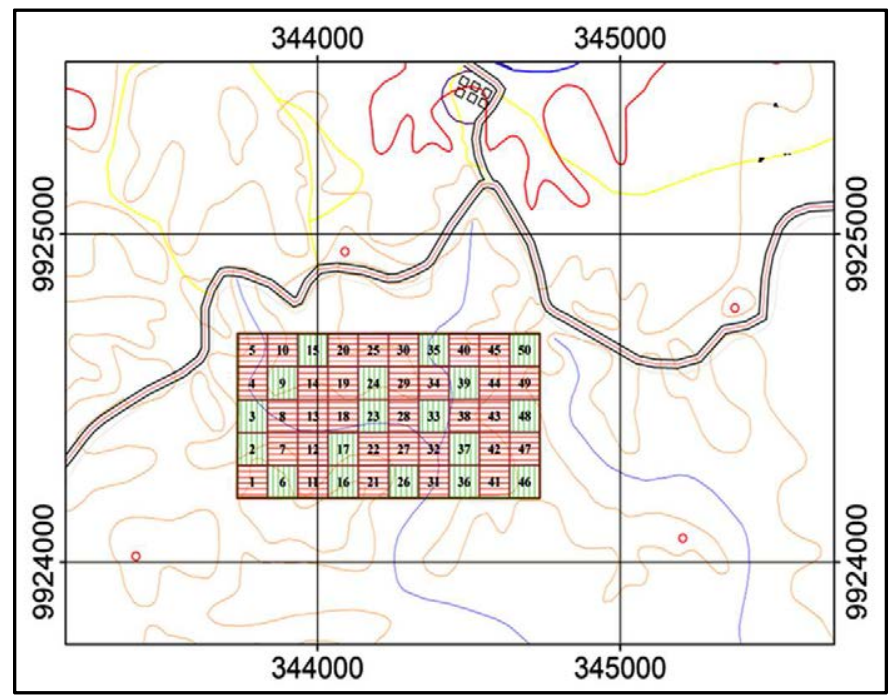

Figura3.32: Malla regular de sub-parcelas de 1-ha usadas para la calibración (entramado rojo) y validación (entramado verde) del modelo general de estimación de ACD usando LiDAR, UTM 18-S SIRGAS.

Se debe considerar que la parcela $\mathrm{N}^{\mathrm{o}} 2$ contiene un remanente de bosque secundario $(0.48$ ha), resultado de la tala de árboles para la construcción de un antiguo helipuerto abierto en épocas de prospección petrolera (alrededor del año 1987). Esta parcela no fue considerada en el ajuste del modelo, pero si en la validación del mismo.

En el proceso de análisis exploratorio de los datos LiDAR se identificaron 2 parcelas: N³6 y $\mathrm{N}^{\circ}$ 50; que albergaban los árboles más grandes de la zona de estudio, y que presentaban los coeficientes de variación y varianza más elevados del conjunto de los datos LiDAR. Estas parcelas fueron analizadas para la validación del modelo.

\subsubsection{Ajuste del modelo}

Para el ajuste del modelo se consideraron solamente los árboles con DAP $\geq 10 \mathrm{~cm}$ (34.567 individuos). En un estudio previo en 25 ha de la parcela, Valencia et al. (2009), encontraron que los árboles con DAP $<10 \mathrm{~cm}$ contribuyen con $\sim 5 \%$ en la colina y $7 \%$ en el valle del total de la AGB en la parcela de estudio. El ajuste y diagnosis del modelo se realizó con el programa R Commander (Fox, 2005).

En la formulación del modelo se utilizó la métrica TCH, ya que presentó menor variabilidad y mejor correlación lineal con las variables AGB y área basal que la métrica MCH. El modelo general se ajustó mediante regresión lineal múltiple por el método de 
mínimos cuadrados, transformando logarítmicamente las variables $\mathrm{ACD}, \mathrm{TCH}, \mathrm{BA}, \mathrm{y}$ $\mathrm{WD}_{B A}$ en la siguiente forma:

$$
\ln (A C D)=\ln a+b_{1} \ln (T C H)+b_{2} \ln (B A)+b_{3} \ln \left(W D_{B A}\right)
$$

Los parámetros estimados del modelo y las medidas de bondad de ajuste se presentan en la tabla 3.12:

Tabla 3.12: Resumen de las estadísticas del ajuste de regresión lineal múltiple.

\begin{tabular}{|c|c|c|c|c|c|c|}
\hline \multirow{2}{*}{ Modelo } & \multicolumn{4}{|c|}{ Parámetros estimados del modelo y error estándar } & \multirow{2}{*}{ RMSE } & \multirow{2}{*}{$\mathbf{R}^{2}$ corregido } \\
\hline & $a$ & $b_{1}$ & $b_{2}$ & $b_{3}$ & & \\
\hline $\operatorname{Ln} \mathrm{ACD}=\ln \mathrm{a}+b_{l} \ln (\mathrm{TCH})$ & 0.768 & 0.140 & 1.229 & 0.983 & 0.03182 & 0.966 \\
\hline$+b_{2} \ln (\mathrm{BA})+b_{3} \ln (\mathrm{WD})$ & $(0.291)$ & $(0.087)$ & $(0.073)$ & $(0.252)$ & & \\
\hline
\end{tabular}

Todos los términos fueron significativos $\mathrm{p}<0.05 ; \mathrm{RMSE}=$ raíz cuadrada del error cuadrático medio.

\subsubsection{Diagnosis del modelo}

Después del ajuste, se verificó la validez estadística del modelo, garantizando que cumpla con los principios que confirman las hipótesis básicas sobre la distribución de la variable respuesta, la distribución de los errores del modelo y la relación entre las variables que intervienen en el modelo (Peña Sánchez de Rivera, 2002):

a) Normalidad: las observaciones siguen una distribución normal.

b) Homocedasticidad o igualdad de varianzas.

c) independencia,

d) no colinealidad

e) ausencia de valores atípicos.

El modelo final cumplió con todas las hipótesis planteadas. Finalmente, se realizó un análisis gráfico de los residuos (figura 3.33): 


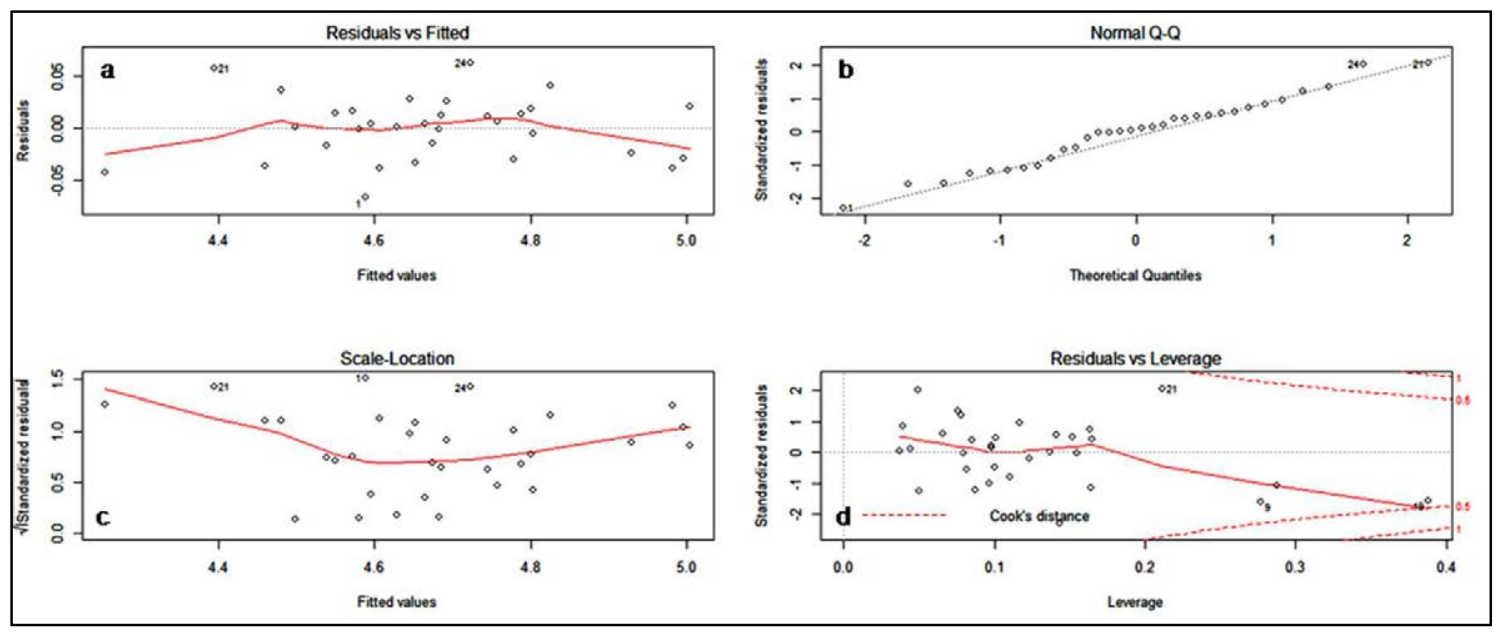

Figura 3.33: Análisis gráfico de los residuos.

El gráfico de los residuos en función de los valores predichos (figura 3.34-a), muestra las dispersiones de los residuos tanto positivas como negativas, y permite evaluar la linealidad y homocedasticidad de los residuos, la situación óptima sería aquella en la que la mayoría de las dispersiones tendiesen a 0. El gráfico Normal Q-Q (figura 3.34-b) permite evaluar la normalidad de los residuos, de forma que se acepta la normalidad si los puntos están alineados. En este caso, se puede apreciar que los puntos se ajustan bien a la recta. La figura 3.34-c analiza la dispersión de los residuos estandarizados en función de los valores predichos; y la figura 3.34-d muestra la distancia de Cook y que observaciones podrían ser potencialmente influyentes en el modelo. En resumen, los 2 primeros gráficos sirven para comprobar la hipótesis de normalidad, homogeneidad de varianza e independencia de errores que se asumen al ajustar una relación empleando regresión por el método de mínimos cuadrados; y los 2 siguientes permiten analizar si existe alguna tendencia que pueda indicar una relación entre variables que no haya sido tenida en cuenta en el modelo.

Por tanto, se puede aceptar que un modelo que explica el $96 \%$ de la variación de ACD a través de la variable TCH obtenida a partir de datos LiDAR, y las observaciones de campo (AB $y$ WD) para bosque tropical en la Amazonía Occidental tendría la forma siguiente:

$$
\operatorname{Ln} A C D=\ln (0.768)+0.140 \ln (A B)+0.983(W D)
$$

La transformación logarítmica introduce un sesgo (subestimación) en los cálculos (Baskerville, 1972). Para eliminar este sesgo, el resultado final debe ser multiplicado por un factor de corrección (FC), que se calcula a partir del error estándar de la estimación. El modelo final se multiplica por este factor de corrección, en este caso $=1.00044$ del sesgo 
sistemático.

Destransformamos la ecuación ya que nos interesa el parámetro ACD por ha y no su ln, corregimos el sesgo sistemático generado por la linealización y la ecuación queda expresada de la siguiente manera:

$$
A C D=2.15813 * T C H^{0.14015} B A^{1.2292} W D^{0.9839}
$$




\section{$\varnothing 4$}

\section{ANÁLISIS DE RESULTADOS Y DISCUSIÓN}

“Los árboles son parte de la solución contra el cambio climático, el mayor problema que hemos enfrentado nunca.

Vandana Shiva 


\section{4. $\quad$ ANÁLISIS DE RESULTADOS Y DISCUSIÓN}

\subsection{Validación del modelo}

Un procedimiento riguroso de validación de un modelo consiste en verificar los resultados con una muestra independiente de la utilizada para construirlo. Debido a que la bondad del ajuste no refleja necesariamente la bondad de una predicción futura, para la validación se utilizaron 18 subparcelas que fueron seleccionadas para tal fin. Este procedimiento permitió una evaluación más robusta del modelo general. Los criterios para comparar las predicciones a las observaciones, son equivalentes a los utilizados para evaluar la calidad del ajuste del modelo. Cuando se aplicó el modelo ajustado a las parcelas de validación, se obtuvieron los resultados que se muestran en la figura 4.1: 


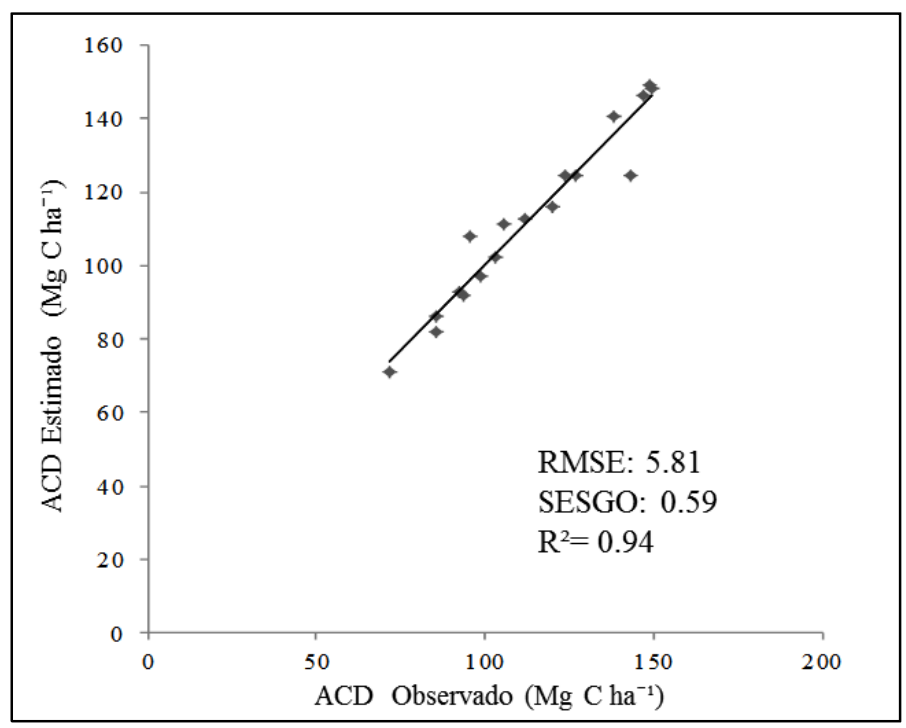

Figura 4.1: Validación del modelo general de ACD en 18 subparcelas independientes.

Los resultados de la validación directa ponen de manifiesto que las estimaciones de ACD usando el modelo general son precisas $\left(\mathrm{RMSE}=5.81 \mathrm{Mg} \mathrm{C} \mathrm{ha}^{-1}\right)$, y presentan una alta consistencia con las medidas de campo $\left(\mathrm{R}^{2}=0.94\right)$, sin apenas desviaciones sistemáticas (sesgo próximo a 0), recogiendo la variabilidad de los 2 ecosistemas en la zona de estudio.

\subsection{Validación del modelo por posiciones topográficas}

En la figura 4.2 se presenta el desempeño del modelo ajustado para cada una de las posiciones topográficas. Los resultados muestran que el modelo es espacialmente sensible a las variaciones de ACD en las 3 posiciones topográficas. La selección de subparcelas para el ajuste del modelo considerando estas posiciones, incide en la precisión de las estimaciones de ACD en la zona de estudio. El sesgo en cada posición topográfica proporciona un conocimiento general de cómo las parcelas de campo representan el paisaje. La disminución del sesgo es independiente del número de parcelas de calibración; sólo depende del error de muestreo asociado a estas parcelas, que está determinado en gran medida por el tamaño de la parcela (Réjou-Méchain et al., 2014).

La mayor diferencia entre las observaciones de campo y las estimadas por el modelo se presenta en la cima que contiene el remanente de bosque secundario (figura 4.2-c). Esta diferencia se debe a 2 situaciones muy concretas: a) el mayor crecimiento de los árboles del bosque secundario en comparación con los árboles del bosque maduro en la parcela; y b) el tiempo transcurridos entre el censo de árboles y la toma de datos LiDAR. La figura 4.2-d 
muestra la validación del modelo sin la parcela 2, donde se puede comprobar la mejora en la precisión del modelo.

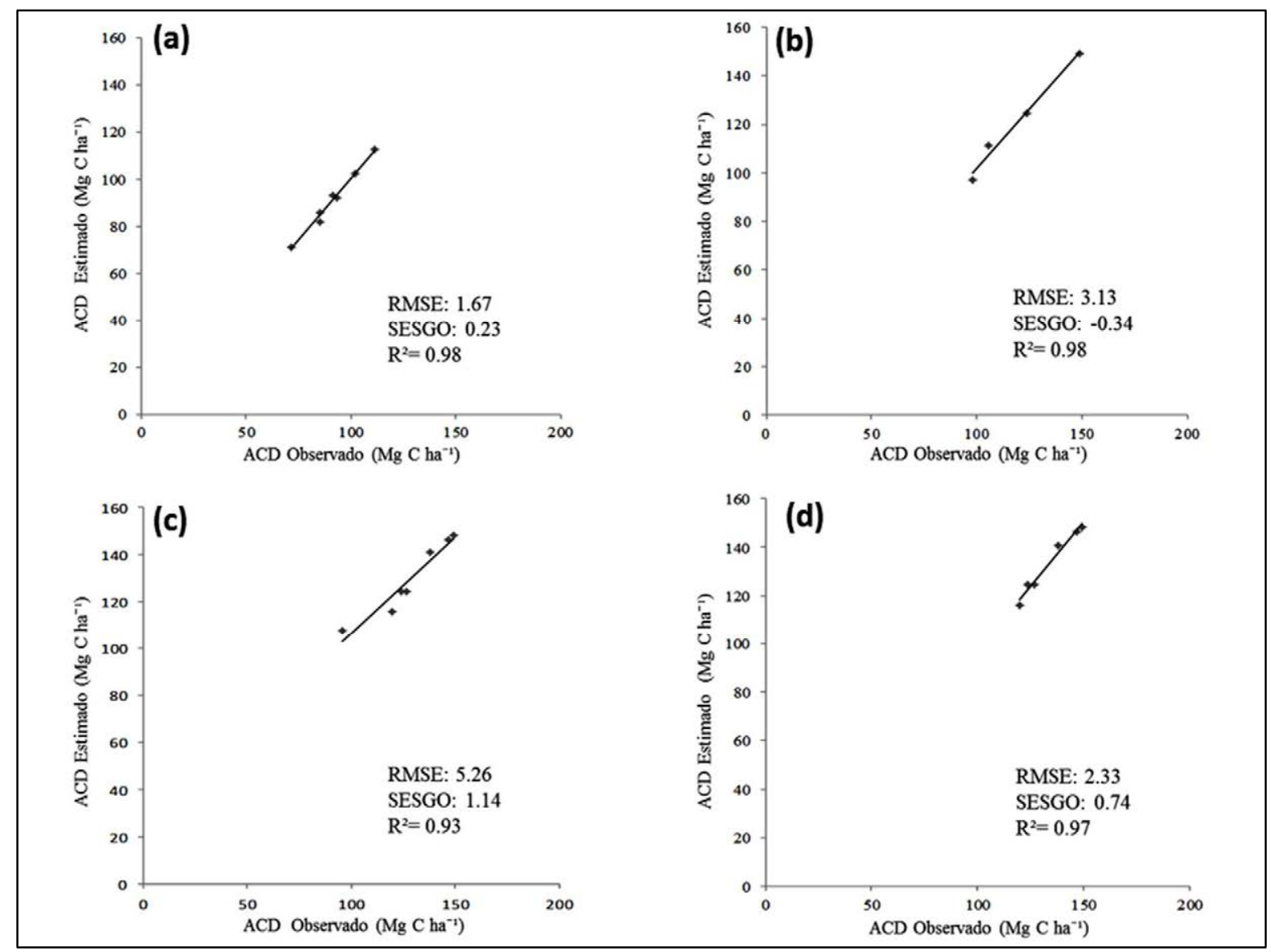

Figura 4.2: Validación del modelo general de ACD por posiciones topográficas en: (a) valle; (b) ladera; (c) cima incluyendo el remanente de bosque secundario; (d) cima sin bosque secundario.

\subsection{Precisión de las estimaciones de ACD con el modelo local}

Para la validación del enfoque general aplicado en esta tesis, Asner et al. (2012) utilizaron 50 parcelas, con un tamaño entre 0.8-1.0 ha, localizadas en Panamá y Perú. Los resultados del ajuste obtuvieron un $\mathrm{R}^{2}$ corregido $=0,86$ y un $\mathrm{RMSE}=13.2 \mathrm{Mg} \mathrm{C}^{-1}$. Con el modelo local, utilizando 18 parcelas de 1 ha y considerando un muestreo estratificado por posiciones topográficas, resultó un $\mathrm{R}^{2}$ corregido $=0,94$ y un $\mathrm{RMSE}=5.81 \mathrm{Mg} \mathrm{C}^{-1}$. Los valores altos del coeficiente de determinación $\left(\mathrm{R}^{2}\right)$ corregido se pueden atribuir a la influencia del tamaño de la parcela y de la densidad de puntos LiDAR (Ruiz et al., 2014).

Si bien en esta investigación no se realizó un análisis de la densidad de puntos LiDAR y 
su influencia en la estimación del ACD, un estudio reciente de Leitold et al. (2015) realizado en bosque tropical de Brasil, ha llegado a obtener errores de 80 a $125 \mathrm{Mg} \mathrm{ha}^{-1}$ en las predicciones de AGB para densidades de retorno LiDAR por debajo de los 4 puntos $\mathrm{m}^{-2}$. Las alturas de dosel arbóreo calculadas con LiDAR, disminuyeron a medida que se reducía la densidad de puntos, debido a un efecto sistemático en la construcción de modelo digital de superficie. El estudio de Leitold et al. (2015) sugiere que se ha de tener cierta precaución al utilizar modelos generalizados de ACD que utilicen una sola métrica LiDAR, como en este caso de estudio, sobre todo a bajas densidades de retorno LiDAR. Sin embargo, la densidad de puntos alcanzada en esta tesis, mitiga el problema de la precisión de la altura del dosel y la generación del MDT. En contraste con el estudio realizado por Leitold et al. (2015); Hansen et al. (2015), en un estudio realizado en bosque tropical en Tanzania, pusieron de manifiesto que se podían utilizar las métricas de dosel obtenidas a partir de bajas densidades de puntos láser, para la estimación de ACD. Los resultados obtenidos sugieren que se debe realizar más investigaciones destinadas a evaluar la influencia de la densidad de puntos LiDAR en la generación del MDT y de las métricas de dosel, especialmente en entornos tropicales. En esta tesis, la diferencia media en elevación (-0.722 m) entre el MDT generado con LiDAR y las observaciones GNSS en la parcela, indicaron una ligera subestimación de la elevación en el MDT final.

En la fase de calibración entre las estimaciones de campo y las obtenidas con LiDAR, el uso de parcelas pequeñas da lugar a errores de dispersión mayores, y en consecuencia el aumento del RMSE entre el TCH (o cualquier métrica) derivada de LiDAR, y las estimaciones en campo de ACD (Asner et al., 2014; Zolkos et al., 2013). Si bien la implementación de parcelas más grandes incrementa los costos y el tiempo para el muestreo en campo, las parcelas grandes permiten generar modelos con mejores resultados, aumentando la precisión de las predicciones de ACD y reduciendo la variación de las métricas derivadas de LiDAR.

Además, es importante tener en cuenta que contar con parcelas grandes, que representen el paisaje, y con mediciones espacialmente explicitas de diámetro y densidad de madera de todos los individuos en el área de estudio; pueden conducir a una comprensión más robusta de las incertidumbres en el proceso de calibración de los modelos. 


\subsection{Modelo para la estimación de área basal a partir de datos LiDAR}

El área basimétrica o área basal, es una medida de masa forestal que permite determinar la superficie que ocupa un determinado grupo de árboles. La estimación del área basal con LiDAR ha sido uno de los parámetros forestales de mayor interés en los bosques de coníferas (Næsset et al., 2004). Sin embargo, se han realizado pocos estudios en bosques tropicales (Drake et al., 2002; Vincent et al., 2012). En los bosques tropicales húmedos, el área basal está fuertemente afectada por factores del paisaje local, pero es relativamente invariante a escala regional (Malhi et al., 2006). Las métricas de altura de vegetación derivadas de LiDAR podrían servir también para la estimación directa de área basal en los bosques tropicales. Sin embargo, se ha evidenciado que la relación entre el área basal y las métricas de altura de dosel derivadas de LiDAR varían significativamente entre tipos de bosques a nivel de paisaje local (Vincent et al., 2012), así como entre regiones (Asner et al., 2012a).

Los bosques tropicales muestran diferencias ecorregionales en las relaciones alturadiámetro de árbol y de densidad de la madera, y estas diferencias afectan a las comparaciones de alometría de parcela agregada con las métricas LiDAR. Asner et al.(2012a), encontraron que el área basal a nivel de parcela está correlacionada con el TCH, lo que también se ha comprobado en la presente tesis doctoral (coeficiente de correlación de Pearson's $r=0.6707$ ); y que el área basal a nivel de parcela puede ser estimada utilizando una conversion lineal específica entre el TCH y el área basal para cada región. Esta relación, denominada "stocking coefficient" (SC), se mantiene estable dentro de regiones, y a menudo a través de un rango amplio de condiciones ambientales. En su estudio Asner et al.(2012a) encontraron que los valores del SC para los bosques de tierras bajas en Panamá, Colombia y la Amazonía Peruana varian desde 1.13 a 1.34.

Siguiendo el mismo enfoque, se utilizó regresión lineal para estimar la relación regional (SC) entre el área basal y la TCH (figura 4.3), usando las estimaciones realizadas en las 32 subparcelas. 


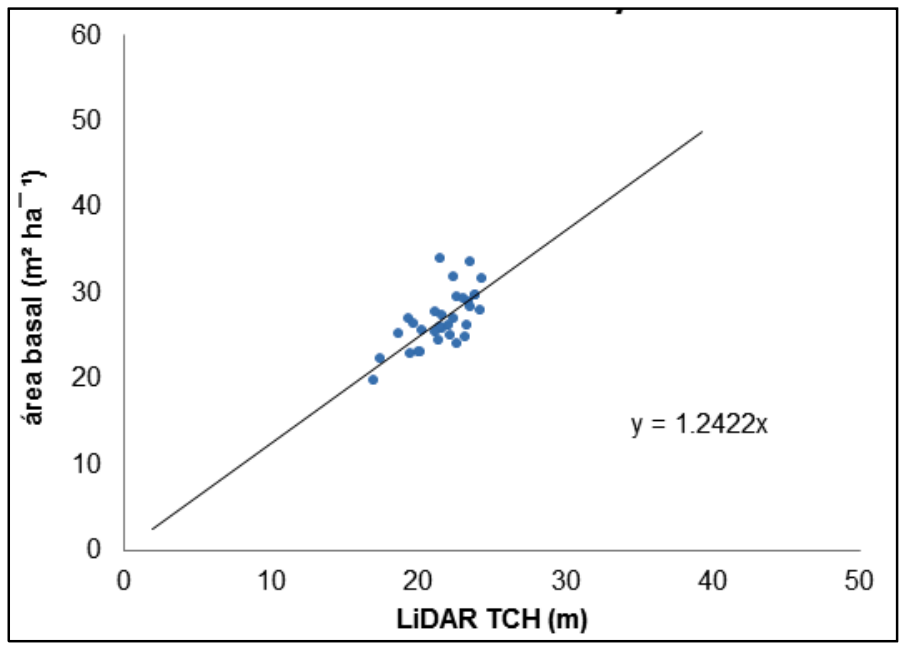

Figura 4.3: Relación entre el área basal estimada en campo y la altura media al tope del dosel (TCH) obtenida con LiDAR en la zona de estudio.

El modelo que establece la relación entre el área basal y el TCH quedó definido de la siguiente manera:

$$
A B=1.2422 * T C H
$$

El valor del $\mathrm{SC}=1.2422$, se encuentra en el rango de los valores obtenidos en regiones neotropicales en los estudios anteriores llevados a cabo por Asner et al. (2012a).

\subsection{Cálculo de la densidad de madera a partir de datos LiDAR}

Los sistemas LiDAR no pueden detectar diferencias en la densidad de la madera, y, por lo tanto, la medida en que la densidad de la madera influye en la variación de ACD, constituye un desafío en el uso de LiDAR para el cartografiado de carbono forestal en bosques tropicales. La falta de una relación entre la densidad de la madera y la TCH es explicado a detalle por Asner et al. (2012a).

Al igual que con el área basal, Asner et al. (2012a) proponen realizar calibraciones regionales de densidad de madera a partir de la relación con la altura media al tope de dosel (TCH); especialmente para zonas extensas de cobertura LiDAR y con grandes variaciones en altitud. Dado que la zona de cobertura LiDAR en esta investigación es relativamente pequeña, que la variación altitudinal es baja (219 - 330 m.), y tomando en cuenta que la parcela de estudio es una muestra representativa del paisaje, se utilizó la densidad de madera promedio obtenida en las estimaciones hechas en campo, su valor es igual a $0.5646 \mathrm{gr} \mathrm{cm}^{-3}$. 


\subsection{Aplicación del modelo general de estimación de ACD en otras zonas de estudio}

Para aplicar el modelo general propuesto en otras zonas de estudio, no es necesario realizar un inventario exhaustivo. En su lugar, y de manera más simple, este enfoque utiliza un único coeficiente de almacenamiento o SC (la relación de BA a TCH), y una sola densidad de la madera constante para cada región basado en la literatura existente. Basta con realizar estimaciones de BA (por relascopio o método prisma), y WD (mediante el registro de las especies dominantes), en puntos espacialmente explícitos dentro de la cobertura de vuelo LiDAR donde se ha obtenido el TCH. A continuación, por regresión de BA y WD en $\mathrm{TCH}$, y sustituyendo estas regresiones en el modelo general, se pueden generar predicciones regionales para el área de interés. Las referencias a estudios en bosques tropicales que utilizan este enfoque se han citado en la Introducción.

\subsection{Modelo final para la estimación de ACD en la zona de cobertura LiDAR}

La inclusión de la calibración local (SC) del área basal y la densidad de la madera en el modelo ajustado (ecuación 3.10), lleva a la formulación de la siguiente ecuación final (ecuación 3.11) para el modelado del ACD con LiDAR en la zona de estudio:

$$
\begin{gathered}
A C D=2.15813 * T C H^{0.14015}(1.2422 * T C H)^{1.2292}(0.5646)^{0.9839} \\
A C D=1.2297 * T C H^{0.14015}(1.2422 * T C H)^{1.2292}
\end{gathered}
$$

\subsection{Geografía del carbono en la zona de estudio}

Los mapas de biomasa y contenido de carbono sobre el suelo de los bosques tropicales son importantes para la conservación de los bosques, ya que constituyen un material básico para la elaboración de políticas de mitigación del cambio climático, y la comprensión del papel que desempeñan los bosques tropicales en el ciclo global del carbono.

Para obtener el mapa de alta resolución de las reservas de carbono en el área de estudio, se aplicó la ecuación 3.11 a toda la superficie de cobertura LiDAR utilizando algebra de 
mapas. La zona total de cobertura LiDAR se dividió en unidades de análisis de 1 hectárea (malla regular de celdas cuadradas -fishnet), y se calculó el TCH (figura 4.4 y anexo F) a partir del modelo de digital de superficie normalizado (nMDS).

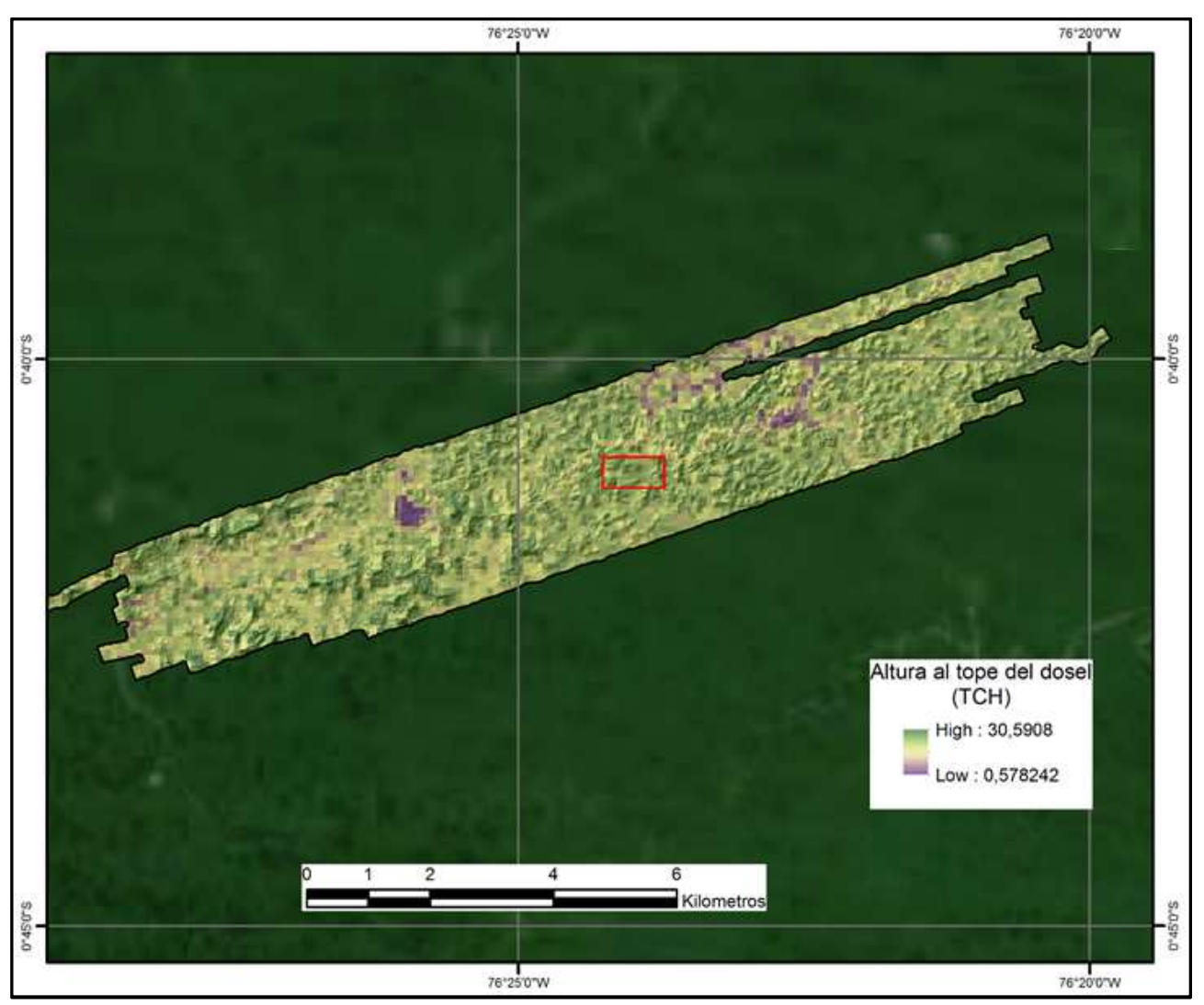

Figura 4.4: Mapa de altura media al tope del dosel (TCH) para la zona de estudio.

El mapa resultante de las reservas de carbono sobre el suelo (figura 4.5 y anexo G), a pesar de cubrir una pequeña extensión de territorio, es una muestra representativa de la diversidad forestal del Parque Nacional Yasuní. El mapa muestra valores de densidad de carbono para los 2 ecosistemas en la zona de estudio. 


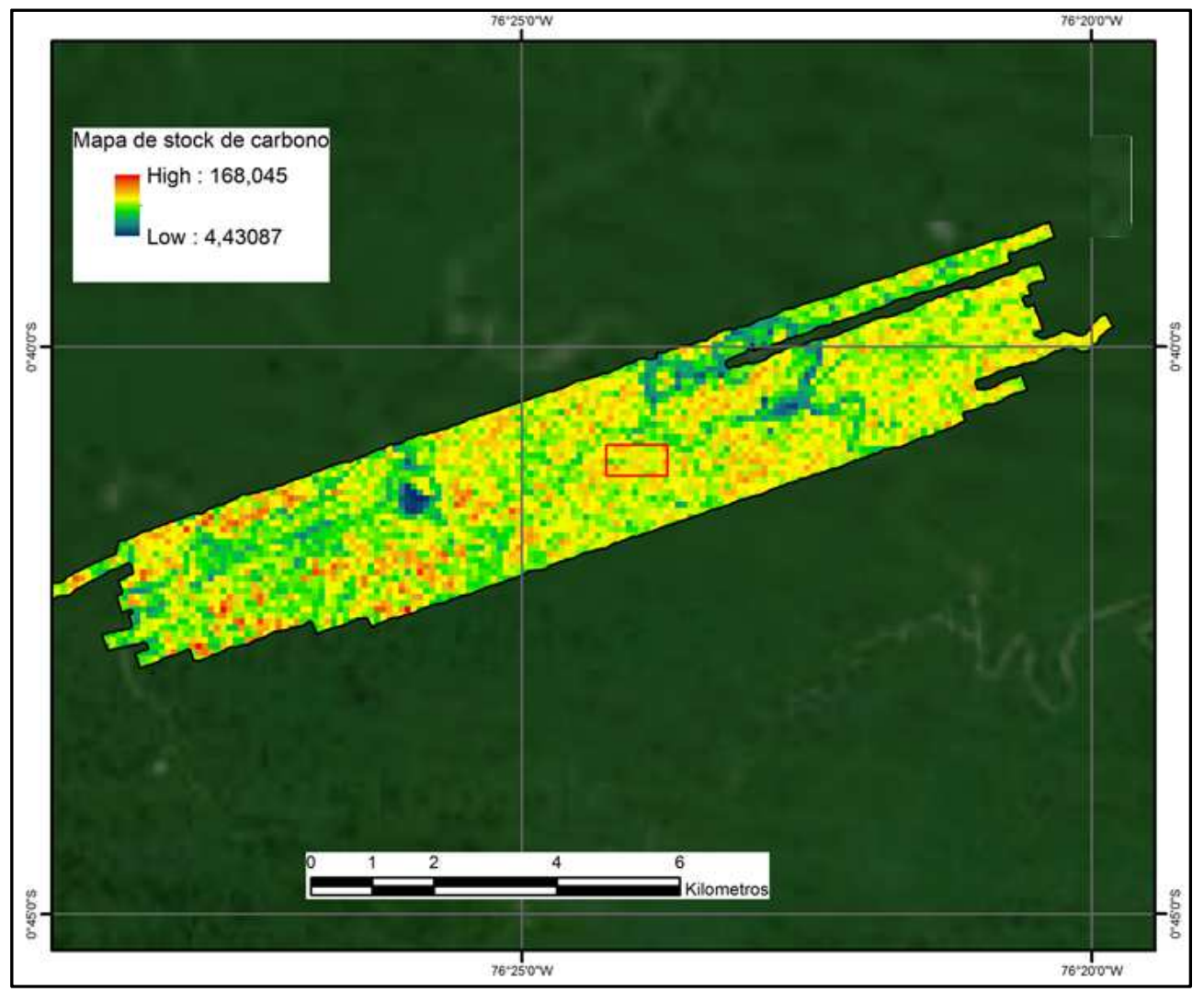

Figura 4.5: Mapa de las reservas de carbono sobre el suelo en la zona de estudio.

Los valores más altos de $\mathrm{ACD}\left(168 \mathrm{Mg} \mathrm{C} \mathrm{ha}^{-1}\right)$ se localizan en los bosques ubicados en los sitios más elevados (cimas), mientras que las menores densidades de ACD se localizan en los bosques en los valles ( $82 \mathrm{Mg} \mathrm{C} \mathrm{ha}^{-1}$ ), y en zonas inundadas, o propensas a inundaciones (47 $\mathrm{Mg} \mathrm{C}^{-1}$ ). Las zonas con almacenamiento bajo de carbono se deben probablemente a las condiciones hidrológicas como la anoxia, y posiblemente a cambios en la fertilidad del suelo asociados con el substrato geológico subyacente (Asner et al., 2010). Los resultados obtenidos concuerdan con el estudio realizado por (CAO y el Ministerio del Ambiente de Perú, 2014) en la Amazonía Norte del Perú, donde encontraron que las mayores densidades de carbono se localizan al norte del río Napo, en el límite fronterizo con el Ecuador.

\subsection{Análisis de la incertidumbre del mapa de carbono}

Los errores en la calibración pueden ser asociados con el mapa de ACD resultante solo si la resolución del mapa (tamaño del pixel), es la misma que el tamaño de la parcela utilizada en la calibración del modelo. Según Mascaro et al. (2011a) existen 3 fuentes de error que pueden influir en la relación entre las estimaciones de densidad de carbono obtenidas a 
partir de LiDAR y las estimaciones obtenidas en campo:

1. Errores posicionales entre las parcelas de campo y los datos LiDAR.

2. Diferencias temporales entre las mediciones LiDAR y las mediciones de campo.

3. Discrepancias entre las mediciones de campo y las mediciones con LiDAR, acerca de la posición de los árboles o parte de ellos, y si estos árboles están dentro o fuera de la parcela de calibración (efecto de borde de parcela).

Las incidencias de estos errores en el modelo son analizadas a continuación.

\subsubsection{Errores posicionales entre las parcelas de campo y los datos LiDAR}

El diseño de muestreo fue una cuadrícula regular de subparcelas de 1 ha en tamaño. El tamaño fue seleccionado en base a los hallazgos de otras investigaciones sobre la disminución de la incertidumbre entre las estimaciones de campo y las estimaciones con LiDAR a esta resolución, y de la suposición subyacente de que las parcelas de estas dimensiones, son una muestra no sesgada del paisaje. El esquema de cuadrícula regular de 1ha, y la precisión horizontal de las esquinas de la parcela de 50 ha $(0.050 \mathrm{~m})$ obtenidas en el levantamiento planimétrico, ayudaron a reducir los errores de co-registro entre las parcelas de campo y los datos LiDAR.

\subsubsection{Diferencias temporales entre las mediciones LiDAR y las mediciones de campo}

El tiempo transcurrido entre el censo forestal y el vuelo de captura de datos LiDAR puede también introducir errores en el modelo final. Esta es una limitación de la investigación, ya que no se contó con datos actualizados del censo debido a situaciones económicas y de tiempo. Sin embargo, se infiere que el error producido por la diferencia temporal entre las mediciones de campo en la zona de estudio es mínimo. Esta consideración se apoya en el estudio realizado por Valencia et al. (2009) en 25 ha de la parcela, donde se examinó el flujo de AGB en diferentes hábitats, y en todas las clases de diámetro utilizando datos de dos censos separados por un intervalo de tiempo promedio de 6.3 años. Valencia et al. (2009) encontró que el bosque de la parcela perdió pequeños tallos (4,6\%), ganó árboles de gran tamaño $(2,6 \%)$, y ganó biomasa $(0,7 \%)$. El cambio en la 
biomasa se debió enteramente al incremento en el tamaño de los árboles, que conduce a más árboles de dosel y un menor número de árboles jóvenes después de sólo 6 años. Entre hábitats, el mayor incremento en la AGB estaba en el parche de bosque secundario $\left(3,4 \% \mathrm{y}^{-}\right.$ $\left.{ }^{1}\right)$, mientras que el bosque maduro en las colinas y valles tuvo un pequeño aumento $(0,10 \%$ y $0,09 \% \mathrm{y}^{-1}$, respectivamente).

En relación a la diferencia entre los hábitats, el cambio de 6 años en AGB fue casi trivial. Del mismo modo, el cambio en el área basal en los tres censos anteriores al vuelo LiDAR (1997, 2002, 2009) fue insignificante, y representó máximo el 1\% $\left(32,94,32,90,33,25 \mathrm{~m}^{2}\right.$ $\mathrm{ha}^{-1}$, respectivamente). Esto puede cambiar en períodos de tiempo anormales, pero es poco probable que cambie en bosque no estacional como el del Parque Nacional Yasuní. Incluso en años extremos, como el de 2010, tal cambio no mostró un gran impacto en los árboles grandes, a juzgar por un muestreo realizado en el año 2012 ( $<2 \%$ de cambio en el área basal).

De estos resultados se deduce que la variación de las existencias de ACD debido al tiempo que transcurre entre el censo de campo y el vuelo LiDAR son mínimas.

\subsubsection{Discrepancias entre las mediciones de campo y las mediciones con LiDAR sobre que árboles o partes de árboles están en el interior de la parcela de calibración.}

En las parcelas LiDAR, las copas de los árboles se bisecan exactamente en el límite de la parcela, mientras que, en las mediciones en las parcelas de campo, los árboles están considerados dentro de la parcela si y solo si más del $50 \%$ de la base del árbol está contenida dentro de la parcela (Condit, 1998).

Las discrepancias en las mediciones entre los datos LiDAR y las observaciones de campo, es decir, los efectos de bisecar las copas de los árboles en los datos LiDAR frente a la ubicación de los árboles "dentro" o "fuera" de la parcela de campo (efecto de borde), disminuyen a un nivel manejable cuando las parcelas de campo alcanzan una hectárea de tamaño (Mascaro et al., 2011a). Además, según reporta Asner et al., (2014a), la precisión del escalamiento alométrico de parcelas agregadas parece aumentar cuando se promedia sobre más vegetación en parcelas más grandes. Las parcelas más grandes minimizan los efectos de borde de parcela relacionadas con la incertidumbre en la inclusión o exclusión de 
un árbol en la parcela LiDAR, y son probablemente más pronunciados en rodales menos densos (Andersen et al., 2005). 


\section{CONCLUSIONES}

“El futuro de las personas del mundo, el futuro de nuestro planeta, está en sus manos. No podemos darnos el lujo de la indecisión, las medidas a medias o los enfoques graduales. Nuestra meta debe ser una transformación”.

Ban Ki-moon, Secretario general de la $\mathrm{ONU}$. 


\section{CONCLUSIONES}

En esta investigación se aplica un enfoque conceptual propuesto por Asner et al. (2012a) cuyo objetivo principal es vincular las estimaciones de biomasa realizadas en campo con una sola métrica obtenida de LiDAR (altura media al tope del dosel) y estimaciones de área basal y densidades de madera a nivel regional. Este enfoque de escalamiento alométrico de parcelas agregadas propone que, si la estructura del bosque y la distribución espacial de la biomasa siguen patrones consistentes, variables a nivel de parcela como el área basal y la densidad de madera podrían capturar suficiente información sobre la cantidad de biomasa como los inventarios completos de campo.

A través de un estudio de caso, se ha demostrado que el enfoque conceptual propuesto por Asner et al. (2012a) combinado con una estrategia de muestreo estratificado por posiciones topográficas que utiliza variables topográficas derivadas de LiDAR (MDT y TWI), permite mejorar la precisión de las estimaciones obtenidas con LiDAR y generar una geografía de alta resolución de las reservas de carbono sobre el suelo. 
Los resultados presentados responden a las preguntas de investigación planteadas, y validan las hipótesis de partida. Las metodologías utilizadas tanto para el diseño de muestreo estratificado, como para la estimación de ACD, han resultado adecuadas, permitiendo obtener estimaciones precisas sobre la densidad de carbono sobre el suelo en la zona de estudio.

\subsection{Se ha demostrado la alta capacidad de predicción del modelo en los ecosistemas de estudio y se ha validado la métrica altura media al tope del dosel.}

Se comprobó que el enfoque de escalamiento alométrico de parcelas agregadas, que incluye variables como el área basal, la densidad de madera promedio y la altura media al tope del dosel $(\mathrm{TCH})$, permite realizar estimaciones precisas de ACD y conocer patrones espaciales continuos de la distribución de la biomasa aérea y del contenido de carbono en la zona de estudio. Las métricas MCH y TCH fueron evaluadas en esta tesis doctoral. La menor variabilidad de la altura media al tope del dosel $(\mathrm{TCH})$ ratifica el uso de esta variable en el modelo general.

La bondad de ajuste y la validación del modelo general de estimación de ACD utilizado, pone de manifiesto, no solo la aplicabilidad del modelo, sino también, la capacidad de predicción $\left(\mathrm{RMSE}=5,81 \mathrm{Mg} \mathrm{C}^{-1}\right.$; para una densidad promedio de $\mathrm{ACD}$ de $\sim 120 \mathrm{Mg} \mathrm{C}$ $\mathrm{ha}^{-1}$ ). El modelo estimado reconoce la variación de ACD en los 2 principales hábitats (valle y colina), y en los 2 ecosistemas en la zona de estudio.

\subsection{Se concluye que el esquema estratificado por posiciones topográficas es efectivo y permite reducir las incertidumbres en las estimaciones de la densidad de carbono sobre el suelo}

Los resultados obtenidos demuestran que la metodología de muestreo estratificado por posiciones topográficas propuesto, permite calibrar de manera adecuada el modelo general con las estimaciones de ACD en campo, logrando reducir el RMSE y el sesgo. La fuerte correlación encontrada entre la elevación media, el índice topográfico de humedad medio 
con la ACD medida en campo, permitió definir el esquema de estratificación de la muestra por posiciones topográficas, ratificando la importancia de la elevación y el TWI como factor predictor de las existencias de ACD.

Los efectos del relieve topográfico, a escala local, son evidentes en los resultados del mapa de ACD. Los bosques ubicados en zonas con baja capacidad de drenaje albergan en promedio $25 \%$ menos ACD que los bosques bien drenados en las cimas. Los resultados confirman la importancia de la topografía como factor ambiental que controla la variación espacial de la biomasa de los árboles, y que debe considerarse en futuras estrategias de muestreo especialmente en los procesos de calibración con técnicas de sensores remotos.

\subsection{Se ha demostrado la importancia del tamaño de la parcela en el proceso de calibración ya que permite reducir las incertidumbres}

La resolución óptima para el monitoreo de carbono en este estudio es la hectárea, no solo porque reduce las incertidumbres, sino también porque es la unidad más común de tenencia de tierra y de las políticas regulatorias en el país. Las parcelas de campo de 1 ha pueden servir de muestra para la calibración del modelo de estimación de ACD, sólo si la media del paisaje está representada por la media de las parcelas de calibración, y cuando la distribución de esta variable a nivel de paisaje sea normal.

Los resultados demuestran que las estimaciones de densidad de carbono con LIDAR pueden alcanzar niveles de error comparables a las estimaciones basadas en medidas en campo, donde el error de muestreo a escala de paisaje puede ser del 10\% de la media. Estos errores también dependen del tamaño del área muestreada y el tamaño de la parcela utilizada. La coincidencia en la escala espacial entre las parcelas de inventario de campo y la resolución espacial del mapa (1 ha) minimiza los errores del cartografiado.

Las parcelas de calibración de 1 ha reducen la dispersión y por ende el RMSE. Este tamaño de parcela produce una mejor calibración ya que abarcan un amplio rango de niveles de almacenamiento de carbono. Sin embargo, el tamaño de parcela óptimo para la calibración no depende solo del tamaño per se de la parcela, sino también de la relación existente entre los tamaños de las coronas de los árboles dentro de la parcela. El estudio de Goodman et al. (2014) sugiere que la precisión de las estimaciones de biomasa de los 
árboles tropicales mejoraría sustancialmente si se tiene en cuenta la alometría de la corona.

\subsection{Con la metodología propuesta ha sido posible realizar el mapa de densidad de carbono sobre el suelo en la zona de estudio}

El mapa resultante muestra valores de densidad de carbono para los 2 ecosistemas de estudio. El ecosistema "Bosque inundado de la llanura aluvial de la Amazonía" presenta valores bajos de $\mathrm{ACD}$ (desde $47 \mathrm{Mg} \mathrm{C}^{-1}$ ), mientras que los valores más altos se presentan en las cimas (168 $\mathrm{Mg} \mathrm{C} \mathrm{ha}^{-1}$ ). Estas diferencias de ACD están asociadas al mayor número de árboles grandes en las cimas y en menor medida a la densidad especifica de la madera.

Existe una considerable variación de ACD entre ecosistemas en la zona de estudio. La selva baja (menos de $400 \mathrm{msnm}$ ), tiene la misma probabilidad de albergar densidades de carbono muy bajas o altas como se evidencio en el estudio. La diferencia significativa en los stocks de carbono encontrada en el pantano, valle y colina sugiere que los bosques de la selva baja contienen un mosaico de hábitats naturales que podrían explicar en parte la variación encontrada en las reservas de carbono en el Parque Nacional Yasuní. Si bien el estudio se enfoca en un área específica localizada en 2 ecosistemas en la Amazonía del Ecuador, la metodología presentada podría aplicarse a cualquier ecosistema en el Ecuador.

\subsection{Se han descrito los beneficios del cartografiado de ACD de alta precisión}

Desde la perspectiva de monitoreo del carbono forestal y REDD+, y el creciente uso de LIDAR aerotransportado para la gestión y conservación del bosque tropical, los resultados de este estudio ponen de manifiesto la importancia de una planificación cuidadosa y un muestreo consistente, que considere la topografía, para la cuantificación exacta de las reservas de ACD sobre el suelo.

Bajo un programa de REDD+, existen considerables beneficios monetarios por la reducción de la incertidumbre en las estimaciones de carbono. La creación y el precio de los créditos de carbono están vinculados con la precisión de la estimación del carbono forestal. El aumento de la incertidumbre en las estimaciones disminuye el valor monetario del 
carbono de los bosques, e inclusive podrían excluir (o reducir) a los países de la generación de beneficios monetarios por la disminución de la deforestación y la conservación de sus bosques.

Los beneficios del cartografiado de alta precisión no solo se pueden reflejar desde el punto de vista económico, sino también, y quizás el más importante, desde el punto de vista de conservación del bosque tropical, y su contribución a los esfuerzos de mitigación del cambio climático.

\subsection{Aportaciones originales de la investigación}

Entre las aportaciones que se pueden considerar originales en esta tesis se citan:

- Se utilizan variables geomorfométricas, en este caso la elevación y el TWI, derivadas del LiDAR para la formulación de un esquema de muestreo estratificado que considere la topografía. En la revisión de literatura realizada en lo referente a estimaciones con LiDAR, no se ha encontrado ningún estudio que utilice estas variables en los diseños de muestreo y en la selección de parcelas para el ajuste de los modelos.

- Se utiliza el análisis de puntos calientes (hotspot) para identificar la distribución de la ACD. Los estudios revisados realizan este análisis en relación a temas como la delincuencia, la seguridad, los accidentes de tráfico, concentración de metales entre otros, pero no se encontraron estudios destinados a la identificación de patrones de densidad de carbono en bosques tropicales.

- Se calibró un modelo general de estimación de ACD con LiDAR, y se generó un mapa preciso del contenido de carbono sobre el suelo de una parte del Parque Nacional Yasuní. Este modelo puede utilizarse para realizar estimaciones precisas de ACD para todo el Parque.

\subsection{Líneas futuras de investigación}

\section{Uso de algoritmos Random forest}


Esta investigación corrobora que el LiDAR aerotransportado puede utilizarse para extender las estimaciones de carbono sobre el suelo obtenidas en parcelas de campo a escalas espaciales mucho más grandes. Las estimaciones de las reservas de carbono sobre el suelo producidas con LiDAR pueden generarse para grandes extensiones de terreno, una tarea que no podría lograrse con parcelas de campo, sin embargo, existe la limitación del coste de adquisición de datos LiDAR.

Una alternativa para evitar los costes económicos que conlleva los trabajos de levantar grandes extensiones de terreno con LiDAR, son los diseños que utilizan el muestreo en varias etapas, en base a adquisiciones de datos LiDAR aerotransportado en posiciones específicas. Por ejemplo, se pueden volar franjas determinadas (líneas de vuelo), y establecer parcelas de campo dentro de los límites de las franjas, y luego éstas se pueden ampliar a zonas más grandes, utilizando otras fuentes de datos adicionales, como las imágenes satelitales. Un aspecto clave en esta alternativa son los algoritmos de aprendizaje automático, que permiten escalar (a mapas regionales o de cobertura completa de un país) las muestras de LiDAR aerotransportado. Algunos autores han utilizado el algoritmo $R F M L$ (Random Forest Machine Learning) para modelar la relación entre las muestras de carbono de bosque tomadas por LiDAR y un conjunto extenso de datos satelitales. RandomForest es un algoritmo de aprendizaje automático no paramétrico que produce un "bosque" de árboles de decisión basado en entradas al azar de los datos de entrenamiento. En un estudio, Mascaro et al. (2014) demostraron que llevar muestras LiDAR a escala regional es 60\% más preciso con $R F M L$ en comparación con los enfoques de estratificación tradicionales.

\section{Influencia de la densidad de puntos LiDAR en la generación del MDT}

En esta investigación se ha utilizado una densidad de 20 puntos por $\mathrm{m}^{2}$, lo que garantizó la generación de un MDT fiable. Sin embargo, existe un estudio en bosque tropical de la Amazonía en Brasil realizado por Leitold et al. (2015) donde concluyen que densidades de puntos por debajo de $4 \mathrm{~m}^{2}$ conducen al aumento del error en la generación del MDT, lo que resultó en una subestimación de la biomasa forestal. En este mismo tema, Hansen et al. (2015) mostraron que se puede generar un MDT fiable a partir de densidades bajas de puntos, en un estudio de caso en Tanzania. A la vista de estos resultados, se necesita realizar mas investigaciones en este sentido, a fin de evaluar la influencia de la densidad de puntos 
LiDAR en la generación de MDT en entornos tropicales.

\section{Tamaño de copa de los árboles y su relación con el tamaño de parcela}

Los patrones de distribución de ACD identificados con LiDAR a lo largo de los 2 ecosistemas de estudio y la magnitud del efecto del error en el tamaño de la parcela de análisis, pareciera que no se refleja por sí solo en el tamaño de la parcela, sino por el tamaño de la parcela en relación con el tamaño medio de la copa de los árboles, que proporciona la mayor parte de la señal de retorno LiDAR. El tamaño de las parcelas puede ser más manejable en los bosques boreales y templados, pero establecer parcelas grandes en entornos tropicales es más difícil y costoso. Encontrar un equilibrio entre el tamaño de la parcela y el tamaño de la copa de los árboles sigue siendo un problema importante para futuros estudios.

\section{Utilización de sensores hiperespectrales}

La Teledetección hiperespectral aerotransportada es una tecnología que permite caracterizar las coberturas terrestres con gran precisión debido a la alta resolución espacial y espectral. La potencialidad de este tipo de sensores puede ser utilizada para identificar las especies dominantes en las zonas donde se realice la calibración del vuelo LiDAR

Como líneas futuras de investigación se propone el uso de sensores hiperespectrales tanto para la detección de especies como para la clasificación de las mismas.

Importancia de la densidad de la madera y del tipo de suelo en las estimaciones de biomasa

A la vista de los resultados obtenidos y para una profundización en el estudio de los factores que determinan la distribución de la biomasa en bosques tropicales, se propone como nueva línea de investigación el análisis de la variable densidad de la madera de cada especie.

Así mismo sería de gran interés estudiar cómo afecta la tipología de suelos en el modelo predictivo de crecimiento de las distintas especies. en los modelos. 


\subsection{Difusión de la investigación}

Durante el período de investigación doctoral se han difundido resultados parciales y finales a través de publicaciones, conferencias y workshops. Para cumplir con los objetivos planteados en el estudio, se realizaron 2 estancias de investigación en Quito-Ecuador y Stanford-USA. La documentación de respaldo se presenta en el anexo H.

\section{Publicaciones:}

Patricio Xavier Molina, Gregory P. Asner, Mercedes Farjas Abadía, Juan Carlos Ojeda Manrique, Luis Alberto Sánchez Diez y Renato Valencia (2015). Spatially-explicit Testing of a General Aboveground Carbon Density Estimation Model in a Western Amazonian Forest using Airborne LiDAR. Remote Sensing, ISSN 2072-4292 doi: 10.3390/rs8010009

Patricio Xavier Molina, Mercedes Farjas Abadía, Luis Alberto Sánchez Diez, Juan Carlos Ojeda Manrique (2015). Modelo de estimación del contenido de carbono sobre el suelo en bosque húmedo tropical de la Amazonía Occidental usando tecnología LiDAR aerotransportada. Libro de Actas del XVI Congreso de la Asociación Española de Teledetección (AET), Sevilla, 395-398, (Eds. J. Bustamante, R. Díaz-Delgado, D. Aragonéz, I. Afán y D. García), ISBN 978-84-608-1726-0, Sevilla, España.

Patricio Xavier Molina. Uso de sensores LiDAR aerotransportados para estimación de parámetros forestales y generación de cartografía temática para estudios forestales y ecológicos (2014). Revista Técnica Geográfica, 5ta edición, págs. 55-68. Quito, Ecuador. Publicación de difusión científica del Instituto Geográfico Militar del Ecuador, disponible en

http://www.igm.gob.ec/work/files/publicaciones2014/revista_tecnica_IGM2014_final.swf

\section{Conferencias y presentación de ponencias en Congresos:}

Conferencia: "Generación de Cartografía de Interés Forestal y Ecológico en Bosque Tropical Amazónico Utilizando Datos LiDAR e Información Multiespectral”, expuesta en el ciclo de conferencias técnicas organizado por el IGM, el día 13 de mayo de 2014 en la ciudad de Quito, Ecuador. 
Comunicación oral "Modelo de estimación del contenido de carbono sobre el suelo en bosque húmedo tropical de la Amazonía Occidental usando tecnología LiDAR aerotransportada. XVI Congreso de la Asociación Española de Teledetección (AET). Sevilla, España, 21 octubre 2015.

\section{Talleres-Workshop:}

"Uso de Herramientas geoespaciales para el mapeo de ecosistemas" desarrollado por el IGM el 09 de septiembre de 2014 en el Centro Panamericano de Estudios e Investigaciones Geográficas (CEPEIGE) en Quito-Ecuador en coordinación con el Carnegie Institution for Science.

\section{Estancias-pasantías de Investigación:}

Pasantía en la Universidad de las Fuerzas Armadas-ESPE, desde el 22 de febrero al 14 de mayo de 2014, Quito-Ecuador.

Estancia de investigación en el Laboratorio del Dr. Greg Asner del Departamento de Ecología Global del Carnegie Institution for Science en la Universidad de Stanford-USA desde el 05 de junio al 30 de julio de 2014, Stanford-USA. 


\section{BIBLIOGRAFÍA}

Agresta S. Coop. (2015). Módulo 5: Métodos de predicción de variables forestales con $R$. [Material de clase]. Curso QGis y LiDAR en la evaluación de ecosistemas forestales, Edic.1. Agresta S. Coop, Madrid, España.

Aldred, A. H., y Bonnor, G. M. (1985). Application of airborne lasers to forest surveys. Ministry of State for Forestry, Petawawa National Forest Institute. (Vol. PI-X.51). Chalk River ON.

Andersen, H.E., McGaughey, R. J., y Reutebuch, S. E. (2005). Estimating forest canopy fuel parameters using LIDAR data. Remote Sensing of Environment, 94(4), 441-449.

Anderson-Teixeira, K. J., Davies, S. J., Bennett, A. C., Gonzalez-Akre, E. B., MullerLandau, H. C., Joseph Wright, S., ... Zimmerman, J. (2015). CTFS-ForestGEO: a worldwide network monitoring forests in an era of global change. Global Change Biology, 21(2), 528-549. doi:10.1111/gcb.12712.

Angelsen, A. (Ed.). (2008). Moving Ahead with REDD Issues, options and Implications. Bogor, Indonesia: Center for International Forestry Research (CIFOR)

Arp, H., y Tranarg, C. A. (1982). Mapping in tropical forests: a new approach using the laser APR [Airborne Profile Recorder]. Photogrammetric Engineering and Remote Sensing, 48.

Arranz, J. (2013). Diseño, optimización y análisis de sistemas basados en técnicas láser, para el modelado geométrico, registro y documentación, aplicados a entidades de interés patrimonial. Tesis Doctoral. Madrid: Universidad Politécnica de Madrid. 499 pp. Disponible en: http://oa.upm.es/15056/1/JOSE_JUAN_ARRANZ_JUSTEL_B.pdf

Asner, G., Hughes, R., Varga, T., Knapp, D. E., \& Kennedy-Bowdoin, T. (2009). Environmental and biotic controls over aboveground biomass throughout a tropical rain forest. Ecosystems, 12, 261-278. doi: 10.1007/s10021-008-9221-5.

Asner, G. P., Mascaro, J., Muller-Landau, H. C., Vieilledent, G., Vaudry, R., Rasamoelina, M., Hall, J.S., van Breugel, M. (2012a). A universal airborne LiDAR approach for tropical forest carbon mapping. Oecologia, 168(4), 1147-1160.

Asner, G. P., Clark, J. K., Mascaro, J., Galindo García, G. A., Chadwick, K. D., Navarrete Encinales, D. A., ... Ordóñez, M. F. (2012b). High-resolution Mapping of Forest Carbon Stocks in the Colombian Amazon. Biogeosciences, 9(7), 2683-2696.

Asner, G. P., Mascaro, J., Anderson, C., Knapp, D. E., Martin, R. E., Kennedy-Bowdoin, T., ... Bermingham, E. (2013). High-fidelity national carbon mapping for resource management and REDD+. Carbon Balance and Management, 8(1), 7. doi:10.1186/1750-0680-8-7.

Asner, G. P., y Mascaro, J. (2014a). Mapping tropical forest carbon: Calibrating plot estimates to a simple LiDAR metric. Remote Sensing of Environment, 140, 614-624. doi: 10.1016/j.rse.2013.09.023. 
Asner, G. P., Martin, R. E., Tupayachi, R., Anderson, C. B., Sinca, F., Carranza-Jimenez, L., y Martinez, P. (2014b). Amazonian functional diversity from forest canopy chemical assembly. Proceedings of the National Academy of Sciences, 111(15), 5604-5609. doi:10.1073/pnas.1401181111.

Asner, G. P., Powell, G. V. N., Mascaro, J., Knapp, D. E., Clark, J. K., Jacobson, J., ... Hughes, R. F. (2010). High-resolution forest carbon stocks and emissions in the Amazon. Proceedings of the National Academy of Sciences of the United States of America, 107(38), 16738-42. doi: 10.1073/pnas.1004875107.

Axelsson, P. (2000). DEM generation from laser scanner data using adaptive TIN models. International Archives of Photogrammetry and Remote Sensing, XXXIII, 110-117.

Baccini, A., \& Asner, G. P. (2013). Improving pantropical forest carbon maps with airborne LiDAR sampling. Carbon Management, 4 (6), 591-600. doi: 10.4155/cmt.13.66.

Baldeck, C. A., Harms, K. E., Yavitt, J. B., John, R., Turner, B. L., Valencia, R., ... у Thomas, D. W. (2013). Soil resources and topography shape local tree community structure in tropical forests. Proceedings of the Royal Society of London B: Biological Sciences, 280(1753), 20122532. doi: 10.1098/rspb.2012.2532.

Baltsavias, E. P. (1999). Airborne laser scanning: basic relations and formulas. ISPRS Journal of Photogrammetry and Remote Sensing, 54 (2-3), 199-214.

Baskerville, G. L. (1972). Use of logarithmic Regression in the Estimation of Plant Biomass. Canadian Journal of Forest Research, (2), 49-53.

Bass, M. S., Finer, M., Jenkins, C. N., Kreft, H., Cisneros-Heredia, D. F., McCracken, S. F., ... Kunz, T. H. (2010). Global conservation significance of Ecuador's Yasuní National Park. PLoS ONE, 5(1), e8767. doi: 10.1371/journal.pone.0008767.

Bater, C. W., \& Coops, N. C. (2009). Evaluating error associated with LiDAR-derived DEM interpolation. Computers and Geosciences, 35(2), 289-300. doi: 10.1016/j.cageo.2008.09.001.

Beven, K., \& Kirkby, M. (1979). A physically based, variable contributing area model of basin hydrology/Un modèle à base physique de zone d'appel variable de l'hydrologie du bassin versant. Hydrological Sciences Bulletin, 24:1, 43-69. doi: $10.1080 / 02626667909491834$.

Brown, S. 1997. Estimating biomass and biomass change of tropical forests: a primer. In FAO Forestry Papers 134, 55 pp. Rome, Italy., FAO.

Carnegie Airborne Observatory (CAO) y el Ministerio del Ambiente del Perú (2014). The High-Resolution Carbon Geography of Perú. Recuperado de https://cao.carnegiescience.edu/publication/

CCBA. (2013). Estándares de Clima, Comunidad y Biodiversidad Tercera edición. Arlington, VA, USA. 
Chasmer, L., \& Hopkinson, C. (2006). Examining the influence of changing laser pulse repetition frequencies on conifer forest canopy returns. Photogrammetric Engineering \& Remote Sensing, 72(12), 1359-1367.

Chave, J., Andalo, C., Brown, S., Cairns, M. A., Chambers, J. Q., Eamus, D., ... Yamakura, T. (2005). Tree allometry and improved estimation of carbon stocks and balance in tropical forests. Oecologia, 145(1), 87-99. doi: 10.1007/s00442-005-0100-x.

Chave, J., Condit, R., Aguilar, S., Hernandez, A., Lao, S., \& Perez, R. (2004). Error propagation and scaling for tropical forest biomass estimates. Philosophical Transaction of the Royal Society B: Biological Sciences, 359(1443), 409-420. doi: 10.1098/rstb.2003.1425.

Chave, J., Condit, R., Lao, S., Caspersen, J. P., Foster, R. B., \& Hubbell, S. P. (2003). Spatial and temporal variation of biomass in a tropical forest: results from a large census plot in Panama. Journal of Ecology, 91(2), 240-252.

Chave, J., Réjou-Méchain, M., Búrquez, A., Chidumayo, E., Colgan, M. S., Delitti, W. B. C., ... Vieilledent, G. (2014). Improved allometric models to estimate the aboveground biomass of tropical trees. Global Change Biology, 1-14. doi: 10.1111/gcb.12629.

Clark, M. L., Clark, D. B., y Roberts, D. A. (2004). Small-footprint LiDAR estimation of sub-canopy elevation and tree height in a tropical rain forest landscape. Remote Sensing of Environment, 91(1), 68-89. doi: 10.1016/j.rse.2004.02.008.

Clark, M. L., Roberts, D. A., Ewel, J. J., \& Clark, D. B. (2011). Estimation of tropical rain forest aboveground biomass with small-footprint LiDAR and hyperspectral sensors. Remote Sensing of Environment, 115(11), 2931-2942. doi: 10.1016/j.rse.2010.08.029.

Condit, R. (1998). Tropical forest census plots. Berlin: Springer-Verlag.

Condit, R., Ashton, P. S., Baker, P., Bunyavejchewin, S., Gunatilleke, S., Gunatilleke, N., ... Yamakura, T. (2000). Spatial patterns in the distribution of tropical tree species. Science (New York, N.Y.), 288(5470), 1414-1418.

Cushman, S. A., Gutzweiler, K., Evans, J. S., \& Mcgarigal, K. (2010). The Gradient Paradigm: A Conceptual and Analytical Framework for Landscape Eco. In S. A. Cushman \& F. Huettmann (Eds.), Spatial Complexity, Informatics, and Wildlife Conservation (pp. 83-108). Tokyo: Springer Japan.

De Oliveira, A. A. D. E., \& Mori, S. A. (1999). A central Amazonian terra firme forest. I. High tree species richness on poor soils. Biodiversity and Conservation, 8 (9), pp.12191244.

d'Oliveira, M. V. N., Reutebuch, S. E., McGaughey, R. J., \& Andersen, H.-E. (2012). Estimating forest biomass and identifying low-intensity logging areas using airborne scanning LiDAR in Antimary State Forest, Acre State, Western Brazilian Amazon. Remote Sensing of Environment, 124, 479-491.

de Castilho, C. V., Magnusson, W. E., de Araújo, R. N. O., Luizão, R. C. C., Luizão, F. J., 
Lima, A. P., \& Higuchi, N. (2006). Variation in aboveground tree live biomass in a central Amazonian Forest: Effects of soil and topography. Forest Ecology and Management, 234 (1-3), 85-96.

Demšar, J. (2006). Statistical comparisons of classifiers over multiple data sets. The Journal of Machine Learning Research, 7, 1-30.

Drake, J. B., Dubayah, R. O., Clark, D. B., Knox, R. G., Blair, J. B., Hofton, M. A., ... Prince, S. (2002). Estimation of tropical forest structural characteristics using large-footprint lidar. Remote Sensing of Environment, 79 (2), 305-319.

Drake, J. B., Knox, R. G., Dubayah, R. O., Clark, D. B., Condit, R., Blair, J. B., \& Hofton, M. (2003). Above-ground biomass estimation in closed canopy Neotropical forests using lidar remote sensing: factors. Global Ecology and Biogeography, 147-159.

Dubayah, R. O., \& Drake, J. B. (2000). Lidar remote sensing for forestry. Journal of Forestry, 98 (6), 44-46.

Dupuy, S., Lainé, G., Tassin, J., \& Sarrailh, J.-M. (2013). Characterization of the horizontal structure of the tropical forest canopy using object-based LiDAR and multispectral image analysis. International Journal of Applied Earth Observation and Geoinformation, 25, 7686.

Erdody, T. T. L., \& Moskal, L. M. (2010). Fusion of LiDAR and imagery for estimating forest canopy fuels. Remote Sensing of Environment, 114 (4), 725-737.

Evans, J., Oakleaf, J., Cushman, S., \& Theobald, D. (2014). An ArcGIS Toolbox for surface Gradient and Geomorphometric Modelling, version 2.0-0. [ultimo acceso 08/12/2015]. Disponible en: http://evansmurphy.wix.com/evansspatial.

Fauset, S., Johnson, M. O., Gloor, M., Baker, T. R., Monteagudo M., A., Brienen, R. J. W., ... Phillips, O. L. (2015). Hyperdominance in Amazonian forest carbon cycling. Nature Communications, 6, 6857.

Feldpausch, T. R., Banin, L., Phillips, O. L., Baker, T. R., Lewis, S. L., Quesada, C. a., ... Lloyd, J. (2011). Height-diameter allometry of tropical forest trees. Biogeosciences, 8(5), 1081-1106.

Feldpausch, T. R., Lloyd, J., Lewis, S., Brienen, R., Gloor, M., Montagudo Mendoza, A., ... Phillips, O. L. (2012). Tree height integrated into pan-tropical forest biomass estimates. Biogeosciences, 9(8), 3381-3403.

Felicísimo, A. M. (1994). Modelos digitales del terreno: introducción y aplicaciones a las ciencias ambientales. 122 pp. Universidad de Oviedo.

Fox, J. (2005). The R Commander: A basic-Statistics Graphical User interface to R. Journal of Statistical Software, 14(9), 1-42.

Frazer, G., Magnussen, S., Wulder, M. A., \& Niemann, K. O. (2011). Simulated impact of sample plot size and co-registration error on the accuracy and uncertainty of LiDAR-derived estimates of forest stand biomass. Remote Sensing of Environment, 115(2), 636-649. 
García, M., Riaño, D., Chuvieco, E., \& Danson, F. M. (2010). Estimating biomass carbon stocks for a Mediterranean forest in central Spain using LiDAR height and intensity data. Remote Sensing of Environment, 114 (4), 816-830.

Gentry, A. H. (1992). Tropical Forest Biodiversity: Distributional Patterns and Their Conservational Significance. Oikos, 63, 19-28.

Getis, A., \& Ord, J. K. (1992). The Analysis of Spatial Association. Geographical Analysis, 24 (3), 189-206.

Goetz, S. J., Baccini, A., Laporte, N. T., Johns, T., Walker, W., Kellndorfer, J., ... Sun, M. (2009). Mapping and monitoring carbon stocks with satellite observations: a comparison of methods. Carbon Balance and Management, 4, 2.

Goodman, R. C., Phillips, O. L., \& Baker, T. R. (2014). The importance of crown dimensions to improve tropical tree biomass estimates. Ecological Applications: A Publication of the Ecological Society of America, 24 (4), 680-98.

Grassi, G., Monni, S., Federici, S., Achard, F., \& Mollicone, D. (2008). Applying the conservativeness principle to REDD to deal with the uncertainties of the estimates. Environmental Research Letters, 3 (3), 035005.

Griffith, D. A. (1987). Spatial autocorrelation: A primer. (Washington, DC: Association of American Geographers).

Gruber, S., \& Peckham, S. (2009). Land-surface parameters and objects in hydrology. Developments in Soil Science, 33 (C), 171-194.

Hansen, E., Gobakken, T., \& Næsset, E. (2015). Effects of Pulse Density on Digital Terrain Models and Canopy Metrics Using Airborne Laser Scanning in a Tropical Rainforest. Remote Sensing, 7 (7), 8453-8468.

Harris, N. L., Brown, S., Hagen, S. C., Saatchi, S. S., Petrova, S., Salas, W., ... Lotsch, A. (2012). Baseline Map of Carbon Emissions from Deforestation in Tropical Regions. Science, 336 (6088), 1573-1576.

Holmgren, P., \& Thuresson, T. (1998). Satellite remote sensing for forestry planning-A review. Scandinavian Journal of Forest Research, 13 (1-4), 90-110.

Hopkinson, C. (2007). The influence of flying altitude, beam divergence, and pulse repetition frequency on laser pulse return intensity and canopy frequency distribution. Canadian Journal of Remote Sensing, 33 (4), 312-324.

Hou, Z., Xu, Q., \& Tokola, T. (2011). Use of ALS, Airborne OR and ALOS AVNIR-2 data for estimating tropical forest attributes in Lao PDR. ISPRS Journal of Photogrammetry and Remote Sensing, 66 (6), 776-786.

Hunter, M. O., Keller, M., Victoria, D., \& Morton, D. C. (2013). Tree height and tropical forest biomass estimation. Biogeosciences, 10 (12), 8385-8399.

Hyyppä, J., Hyyppä, H., Litkey, P., Yu, X., Haggrén, H., Rönnholm, P., .. Maltamo, M. 
(2004). Algorithms and methods of airborne laser scanning for forest measurements. International Archives of Photogrammetry, Remote Sensing and Spatial Information Sciences, 36 (8), 82-89.

Hyyppä, J., Hyyppä, H., Litkey, P., Yu, X., Haggrén, H., Rönnholm, P., ... Maltamo, M. (2004). Algorithms and methods of airborne laser-scanning for forest measurements. International Archives of Photogrammetry, Remote Sensing and Spatial Information Sciences, 36 (8/W2), 82-89.

Hyyppä, J., Hyyppä, H., Yu, X., Kaartinen, H., Kukko, A., \& Holopainen, M. (2009). Forest inventory using small-footprint airborne LiDAR. Topographic Laser Ranging and Scanning: Principles and Processing, 335-370.

Jennings, S. B., Brown, N. D., \& Sheil, D. (1999). Assessing forest canopies and understory illumination: canopy closure, canopy cover and other measures. Forestry, 72 (1), 59-73.

John, R., Dalling, J. W., Harms, K. E., Yavitt, J. B., Stallard, R. F., Mirabello, M., ... Foster, R. B. (2007). Soil nutrients influence spatial distributions of tropical tree species. Proceedings of the National Academy of Sciences of the United States of America, 104 (3), 864-9.

Guevara J, Pitman N, Mogollón H, Cerón C, Palacios W, PMV. (2013a). pp. 178-180 en: Ministerio del Ambiente del Ecuador 2012. Sistema de Clasificación de los Ecosistemas del Ecuador Continental. Ministerio del Ambiente del Ecuador. Quito.

Guevara J, Encarnación F, PMV. (2013b). pp. 169-170 en: Ministerio del Ambiente del Ecuador 2012. Sistema de Clasificación de los Ecosistemas del Ecuador Continental. Ministerio del Ambiente del Ecuador. Quito.

Kanagaraj, R., Wiegand, T., Comita, L. S., \& Huth, A. (2011). Tropical tree species assemblages in topographical habitats change in time and with life stage. Journal of Ecology, 99 (6), 1441-1452.

Köhl, M., Magnussen, S., \& Marchetti, M. (2006). Sampling in Forest Surveys. In Sampling Methods, Remote Sensing and GIS Multiresource Forest Inventory (pp. 71-195). Berlin, Heidelberg: Springer Science \& Business Media.

Kozak, A., \& Kozak, R. (2003). Does cross validation provide additional information in the evaluation of regression models? Canadian Journal of Forest Research, 33 (6), 976-987.

Kraus, K., \& Pfeifer, N. (1998). Determination of terrain models in wooded areas with airborne laser scanner data. ISPRS Journal of Photogrammetry and Remote Sensing, 53 (4), 193-203.

Lefsky, M. A., Cohen, W. B., Harding, D. J., Parker, G. G., Acker, S. A., \& Thomas Gower, S. (2002). Lidar remote sensing of above-ground biomass in three biomes. Global Ecology \& Biogeography, II, 393-399.

Lefsky, M. A., Harding, D., Cohen, W. B., Parker, G., \& Shugart, H. H. (1999). Surface Lidar Remote Sensing of Basal Area and Biomass in Deciduous Forests of Eastern 
Maryland, USA. Remote Sensing of Environment, (67), 83-98.

Leitold, V., Keller, M., Morton, D. C., Cook, B. D., \& Shimabukuro, Y. E. (2015). Airborne lidar-based estimates of tropical forest structure in complex terrain: opportunities and tradeoffs for REDD+. Carbon Balance and Management, 10.

Leiva Gutiérrez, N. (2012). Metodología para el cálculo de la humedad del suelo usando parámetros topográficos (MDE), climáticos y edáficos en un sector del piedemonte depositacional del municipio de Villavicencio. Tesis de Maestría. Bogotá: Universidad Nacional de Colombia. 129 pp. Disponible en: http://www.bdigital.unal.edu.co/8910/1/795068.2012.pdf

Leppänen, V. J., Tokola, T., Maltamo, M., Mehtätalo, L., Pusa, T., \& Mustonen, J. (2008). Automatic delineation of forest stands from LIDAR data. GEOBIA, 05-08.

Lewis, S. L., Lloyd, J., Sitch, S., Mitchard, E. T. A., \& Laurance, W. F. (2009). Changing Ecology of Tropical Forests: Evidence and Drivers. Annual Review of Ecology, Evolution and Systematics, 40, 529-549.

Lim, K., Hopkinson, C., \& Treitz, P. (2008). Examining the effects of sampling point densities. Forestry Chronicle, 84 (6), 876-885.

Lim, K. S., \& Treitz, P. M. (2004). Estimation of above ground forest biomass from airborne discrete return laser scanner data using canopy-based quantile estimators. Scandinavian Journal of Forest Research, 19 (6), 558-570.

Lim, K., Treitz, P., Wulder, M., St-Onge, B., \& Flood, M. (2003). LiDAR remote sensing of forest structure. Progress in Physical Geography, 27 (1), 88-106.

Loarie, S. R., Asner, G. P., \& Field, C. B. (2009). Boosted carbon emissions from Amazon deforestation. Geophysical Research Letters, 36 (June), 1-5.

López A, V., Espíndola, F., Calles, J., \& Ulloa, J. (2013). Atlas “Amazonía Ecuatoriana Bajo Presión." Ecociencia. Quito-Ecuador.

Lu, D., Chen, Q., Wang, G., Moran, E., Batistella, M., Zhang, M., ... Saah, D. (2012). Aboveground Forest Biomass Estimation with Landsat and LiDAR Data and Uncertainty Analysis of the Estimates. International Journal of Forestry Research, 2012 (1), 1-16.

Ministerio del Ambiente del Ecuador (MAE) 2013. Sistema de Clasificación de los Ecosistemas del Ecuador Continental. Subsecretaría de Patrimonio Natural. Quito.

Malhi, Y., Wood, D., Baker, T. R., Wright, J., Phillips, O. L., Cochrane, T., ... Vinceti, B. (2006). The regional variation of aboveground live biomass in old-growth Amazonian forests. Global Change Biology, 12 (7), 1107-1138.

Martin, A. R., \& Thomas, S. C. (2011). A reassessment of carbon content in tropical trees. PloS One, 6 (8), e23533.

Martinez-Casasnovas, J. A. (1999). Modelos Digitales de Terreno: Estructuras de datos y 
aplicaciones en el análisis de formas del terreno y en Edafología. QUADERNS DMACS Núm. 25, Departament de Medi Ambient i Ciències del Sòl, Universitat de Lleida, Lleida. 1a Edición: junio de 1999.

Marvin, D. C., Asner, G. P., Knapp, D. E., Anderson, C. B., Martin, R. E., Sinca, F., \& Tupayachi, R. (2014). Amazonian landscapes and the bias in field studies of forest structure and biomass. Proceedings of the National Academy of Sciences, 111, E5224-E5232.

Mascaro, J., Asner, G. P., Knapp, D. E., Kennedy-Bowdoin, T., Martin, R. E., Anderson, C., ... Chadwick, K. D. (2014). A tale of two "Forests": Random Forest machine learning aids tropical Forest carbon mapping. PLoS ONE, 9 (1), 12-16.

Mascaro, J., Detto, M., Asner, G. P., \& Muller-Landau, H. C. (2011a). Evaluating uncertainty in mapping forest carbon with airborne LiDAR. Remote Sensing of Environment, 115 (12), 3770-3774.

Mascaro, J., Asner, G. P., Muller-Landau, H. C., van Breugel, M., Hall, J., \& Dahlin, K. (2011b). Controls over aboveground forest carbon density on Barro Colorado Island, Panama. Biogeosciences, 8 (6), 1615-1629.

Mauro, F., Valbuena, R., Manzanera, J. A., \& García-Abril, A. (2011). Influence of Global Navigation Satellite System errors in positioning inventory plots for tree-height distribution studies. Canadian Journal of Forest Research, 41 (1), 11-23.

Mauro, F., Valentín-Gamazo, M., García Abril, A., Valbuena Puebla, R., Rodríguez Solano, R., \& De Blas De la Vega, A. (2013). Influencia de errores de posicionamiento GNSS en la estimación de variables dasométricas medias a partir de datos LiDAR. In 6to Congreso Forestal Español (Vol. 6CF01-485, p. 2). Vitoria-Gasteiz: Sociedad Española de Ciencias Forestales.

McGaughey, R. J. (2013). FUSION/LDV: Software for LIDAR data analysis and visualization. US Department of Agriculture, Forest Service, Pacific Northwest Research Station, University of Washington. [ultimo acceso 08/12/2015]. Disponible en: http://forsys.cfr.washington.edu/fusion/fusionlatest.html

Means, J., \& Acker, S. (2000). Predicting forest stand characteristics with airborne scanning lidar. Photogrammetric Engineering \& Remote Sensing, 66 (11), 1367-1371.

Meng, X., Currit, N., \& Zhao, K. (2010). Ground filtering algorithms for airborne LiDAR data: a review of critical issues. Remote Sensing, 2 (3), 833-860.

Meyer, V., Saatchi, S. S. S., Chave, J., Dalling, J. W., Bohlman, S., Fricker, G. A., ... Hubbell, S. (2013). Detecting tropical forest biomass dynamics from repeated airborne LIDAR measurements. Biogeosciences, 10 (8), 5421-5438.

Ministerio del Ambiente del Ecuador. (2013). Sistema de Clasificación de los Ecosistemas del Ecuador Continental. Subsecretaría de Patrimonio Natural. Quito.

Mitchard, E. T. A., Feldpausch, T. R., Brienen, R. J. W., Lopez-Gonzalez, G., Monteagudo, A., Baker, T. R., ... Phillips, O. L. (2014). Markedly divergent estimates of Amazon forest carbon density from ground plots and satellites. Global Ecology and Biogeography, 23 (8), 
935-946.

Moffiet, T., Mengersen, K., Witte, C., King, R., \& Denham, R. (2005). Airborne laser scanning: Exploratory data analysis indicates potential variables for classification of individual trees or forest stands according to species. ISPRS Journal of Photogrammetry and Remote Sensing, 59(5), 289-309.

Moore, I., Gessler, P., Nielsen, G. A., \& Peterson, G. A. (1993). Soil attribute prediction using terrain analysis. Soil Science Society of America Journal, 57 (2), 443-452.

Myers, N., Mittermeier, R., Mittermeier, C., da Fonseca, G. A., \& Kent, J. (2000). Biodiversity hotspots for conservation priorities. Nature. 403, 853-858.

Næsset, E. (2009). Effects of different sensors, flying altitudes, and pulse repetition frequencies on forest canopy metrics and biophysical stand properties derived from smallfootprint. Remote Sensing of Environment, 113 (1), 148-159.

Næsset, E., Gobakken, T., Holmgren, J., Hyyppä, H., Hyyppä, J., Maltamo, M., ... Söderman, U. (2004). Laser scanning of forest resources: The Nordic experience. Scandinavian Journal of Forest Research, 19 (6), 482-499.

Naesset, E., \& Næsset, E. (1997). Determination of mean tree height of forest stands using airborne laser scanner data. ISPRS Journal of Photogrammetry and Remote Sensing, 52 (2), 49-56.

Næsset, E., \& Økland, T. (2002). Estimating tree height and tree crown properties using airborne scanning laser in a boreal nature reserve. Remote Sensing of Environment, 79 (1), $105-115$.

Ngomanda, A., Engone Obiang, N. L., Lebamba, J., Moundounga Mavouroulou, Q., Gomat, H., Mankou, G. S., ... Picard, N. (2014). Site-specific versus pantropical allometric equations: Which option to estimate the biomass of a moist central African forest? Forest Ecology and Management, 312, 1-9.

Niklas, K. (2006). A phyletic perspective on the allometry of plant biomass-partitioning patterns and functionally equivalent organ-categories. New Phytologist.

Ni-Meister, W., Lee, S., Strahler, A. H., Woodcock, C. E., Schaaf, C., Yao, T., ... Blair, J. B. (2010). Assessing general relationships between aboveground biomass and vegetation structure parameters for improved carbon estimate from LiDAR remote sensing. Journal of Geophysical Research, 115, G00E11.

Peña Sánchez de Rivera, D. (2002). Regresión y diseño de experimentos (2da ed.). Madrid, España: Alianza editorial.

Pitman, N. (2000). A large-scale inventory of two Amazonian tree communities. Tesis Doctoral: Duke University. 220 pp.Tesis. Disponible en: http://people.duke.edu/ ncp/Homepage_of_Nigel_Pitman,_Duke_University_NSOE_Cente r_for_Tropical_Conservation,_Amazonian_research_and_conservation/Publications_files/pi 
tmanthesis.pdf

Plugge, D., Baldauf, T., \& Köhl, M. (2013). The global climate change mitigation strategy REDD: monitoring costs and uncertainties jeopardize economic benefits. Climatic Change, 119 (2), 247-259.

Rapidlasso GmbH. (2013). LAStools Rapid LiDAR Processing. [ultimo acceso 08/12/2015]. Disponible en: http://rapidlasso.com/

Réjou-Méchain, M., Muller-Landau, H. C., Detto, M., Thomas, S. C., Le Toan, T., Saatchi, S. S., ... Chave, J. (2014). Local spatial structure of forest biomass and its consequences for remote sensing of carbon stocks. Biogeosciences, 11(4), 5711-5742.

Reutebuch, S. E., Andersen, H.-E., \& McGaughey, R. J. (2005). Light detection and ranging (LIDAR): an emerging tool for multiple resource inventory. Journal of Forestry, 103(6), 286-292.

Romero-Saltos, H., Hernández, C., \& Valencia, R. (2014a). Arboles emblemáticos de Yasuní, Ecuador (1era. ed.). Quito, Ecuador: Publicaciones del Herbario QCA, Escuela de Ciencias Biológicas, Pontificia Universidad Católica del Ecuador.

Romero-Saltos, H., Hernández, C., \& Valencia, R. (2014b). Diversidad y dinámica de árboles en una parcela de gran escala. In Arboles emblemáticos de Yasuní, Ecuador (1era ed., pp. 14-22). Quito: Publicaciones del Herbario QCA. Escuela de Ciencias Biológicas, Pontificia Universidad Católica del Ecuador.

Romero-Saltos, H., Valencia, R., \& Macía, M. J. (2001). Patrones de distribución y rareza de plantas leñosas en el Parque Nacional Yasuní y la Reserva Étnica Huaorani, Amazonía ecuatoriana. In J. F. Duivenvoorden, H. Balslev, J. Cavelier, C. Grandez, H. Tuomisto, \& R. Valencia (Eds.), Evaluación de recursos vegetales no maderables en la Amazonia noroccidental (pp. 131-162). Amsterdam: Universiteit van Amsterdam.

Ruiz, L. A., Hermosilla, T., Mauro, F., \& Godino, M. (2014). Analysis of the influence of plot size and LiDAR density on forest structure attribute estimates. Forests, 5 (5), 936-951.

Saatchi, S. S., Houghton, R. A., Dos Santos Alvalá, R. C., Soares, J. V., \& Yu, Y. (2007). Distribution of aboveground live biomass in the Amazon basin. Global Change Biology, 13, 816-837.

Saatchi, S. S., Harris, N. L., Brown, S., Lefsky, M., Mitchard, E. T., Salas, W., ... Morel, A. (2011). Benchmark map of forest carbon stocks in tropical regions across three continents. Proceedings of the National Academy of Sciences, 108 (24), 9899-9904.

Säynäjoki, R., Maltamo, M., \& Korhonen, K. T. (2008). Forest inventory with sparse resolution Airborne Laser Scanning data - a literature review (Vol. 103).

Shapiro, S. S., \& Wilk, M. B. (1965). An Analysis of Variance Test for Normality (Complete Samples). Biometrika, 52(3/4), 591-611.

Sistema de Referencia Geocéntrico para las Américas-SIRGAS. (2015). [ultimo acceso 
08/12/2015]. Disponible en: http://www.sirgas.org/index.php?id=15

Sithole, G., \& Vosselman, G. (2004). Experimental comparison of filter algorithms for Bare-Earth extraction from airborne laser scanning point clouds. ISPRS Journal of Photogrammetry and Remote Sensing, 59 (1), 85-101.

Skole, D. L., Samek, J. H., \& Smalligan, M. J. (2011). Implications of allometry. Proceedings of the National Academy of Sciences of the United States of America, 108 (4), E12; author reply E13-E14.

Stoker, J. (2009). Volumetric Visualization of multiple-return Lidar Data: Using Voxels. Photogrammetric Engineering \& Remote Sensing, 75, 119-112.

Su, J., \& Bork, E. (2006). Influence of Vegetation, Slope, and Lidar Sampling Angle on DEM Accuracy. Photogrammetric Engineering \& Remote Sensing, 72 (11), 1265-1274.

Taylor, P., Asner, G., Dahlin, K., Anderson, C., Knapp, D., Martin, R., ... Townsend, A. (2015). Landscape-Scale Controls on Aboveground Forest Carbon Stocks on the Osa Peninsula, Costa Rica. Plos One, 10 (6), 1-18.

Tinkham, W. T., Smith, A. M. S., Hoffman, C., Hudak, A. T., Falkowski, M. J., Swanson, M. E., \& Gessler, P. E. (2012). Investigating the influence of LiDAR ground surface errors on the utility of derived forest inventories. Canadian Journal of Forest Research, 42, 413422.

Tobler, W. R. (1970). A computer movie simulating urban growth in the Detroit region. Economic Geography, 46, 234-240.

Tuomisto, H., Poulsen, A. D., Ruokolainen, K., Moran, R. C., Quintana, C., Celi, J., \& Cañas, G. (2003). Linking Floristic Patterns with Soil Heterogeneity and Satellite Imagery in Ecuadorian Amazonia. Ecological Applications, 13 (2), 352-371

USDA-RMRS, Silva, C. A., Hudak, A. T., \& Crookston, N. L. (2015). "Web -LiDAR forest inventory: LASmetrics application.". [ultimo acceso 23/07/2015]. Disponible en: http://forest.moscowfsl.wsu.edu:3838/LiDARLASmetrics/

Valencia, R., Balslev, H., \& Paz y Miño, G. (1994). High tree alpha-diversity in Amazonian Ecuador. Biodiversity and Conservation, 28, 21-28.

Valencia, R., Condit, R., Foster, R. B., Romoleroux, K., Villa Munoz, G., Svenning, J., ... Balslev, H. (2004). Yasuní Forest Dynamics Plot, Ecuador. In E. C. Losos \& E. G. Leigh (Eds.), Tropical forest diversity and dynamism: Findings from a large-scale plot network (pp. 609-628). Chicago: University of Chicago Press.

Valencia, R., Condit, R., Muller-Landau, H. C., Hernandez, C., \& Navarrete, H. (2009). Dissecting biomass dynamics in a large Amazonian forest plot. Journal of Tropical Ecology, 25 (05), 473.

Valencia, R., Foster, R. B., Villa, G., Condit, R., Svenning, J.-C., Hernández, C., ... Balslev, H. (2004). Tree species distributions and local habitat variation in the Amazon: large forest plot in eastern Ecuador. Journal of Ecology, 92 (2), 214-229. 
Vincent, G., Sabatier, D., Blanc, L., Chave, J., Weissenbacher, E., Pélissier, R., ... Couteron, P. (2012). Accuracy of small footprint airborne LiDAR in its predictions of tropical moist forest stand structure. Remote Sensing of Environment, 125 (55), 23-33.

Walker, W., Baccini, A., Nepstad, M., Horning, N., Knight, D., Braun, E., \& Bausch, A. (2011). Guía de campo para la Estimación de Biomasa y Carbono Forestal. Falmouth, Masachusetts, USA: Woods Hole Research Center.

Wang, Y., Weinacker, H., \& Koch, B. (2008). A lidar point cloud based procedure for vertical canopy structure analysis and 3D single tree modelling in forest. Sensors, 8 (6), 3938-3951.

West, G., Brown, J., \& Enquist, B. (1999). A general model for the structure and allometry of plant vascular systems. Nature. 400, 664-667.

Wolf, J., Fricker, G., Meyer, V., Hubbell, S., Gillespie, T., \& Saatchi, S. (2012). Plant Species Richness is Associated with Canopy Height and Topography in a Neotropical Forest. Remote Sensing, 4 (12), 4010-4021.

Xiaoye Liu. (2008). Airborne LiDAR for DEM generation: some critical issues. Progress in Physical Geography, 32, 31-49.

Yu, X. W., Hyyppa, J., Kaartinen, H., \& Maltamo, M. (2004). Automatic detection of harvested trees and determination of forest growth using airborne laser scanning. Remote Sensing of Environment, 90 (4), 451-462.

Zhao, K., Popescu, S., Meng, X., Pang, Y., \& Agca, M. (2011). Characterizing forest canopy structure with lidar composite metrics and machine learning. Remote Sensing of Environment, 115 (8), 1978-1996.

Zimble, D. A., Evans, D. L., Carlson, G. C., Parker, R. C., Grado, S. C., \& Gerard, P. D. (2003). Characterizing vertical forest structure using small-footprint airborne LiDAR. Remote Sensing of Environment, 87 (2), 171-182.

Zolkos, S., Goetz, S., \& Dubayah, R. (2013). A meta-analysis of terrestrial aboveground biomass estimation using lidar remote sensing. Remote Sensing of Environment, 128, 289298. 
ANEXOS 


\section{ANEXO A}

\section{Línea de tiempo de los censos \\ realizados en la parcela de $\mathbf{5 0}$ ha}




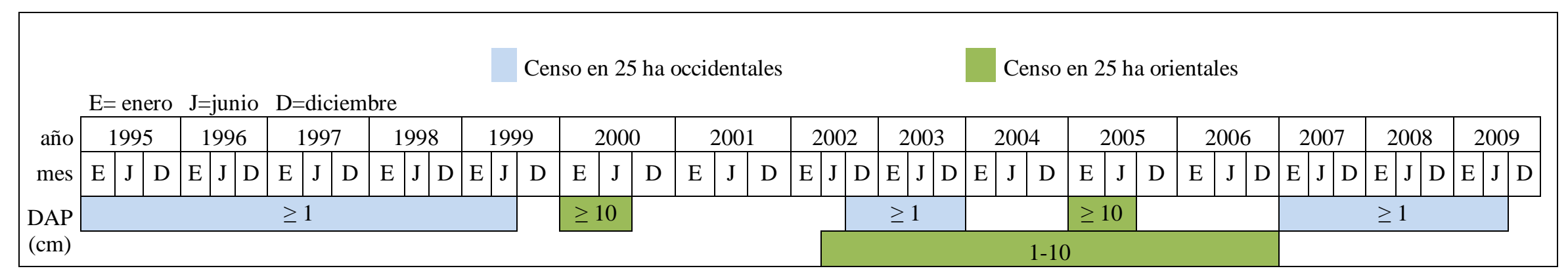

Fuente: Diversidad y Dinámica de árboles en una parcela de gran escala, Romero-Saltos et al. (2014b). 


\section{ANEXO B}

\section{Cuadro-resumen de las estimaciones}

de las variables forestales en campo 


\begin{tabular}{|c|c|c|c|c|}
\hline PARCELA & $\begin{array}{c}\mathrm{N} \geq 10 \mathrm{~cm} \\
\text { DAP }\end{array}$ & $\begin{array}{l}\text { Biomasa } \\
(\mathrm{Mg} / \mathrm{ha})\end{array}$ & $\begin{array}{c}\text { Área Basal } \\
\qquad\left(\mathbf{m}^{2}\right)\end{array}$ & $\begin{array}{c}\text { Densidad_madera } \\
\left(\mathrm{gr} / \mathrm{cm}^{3}\right)\end{array}$ \\
\hline 1 & 736 & 191.36 & 24.81 & 0.5613 \\
\hline 2 & 818 & 199.40 & 30.77 & 0.4720 \\
\hline 3 & 640 & 148.91 & 20.78 & 0.5216 \\
\hline 4 & 686 & 206.99 & 25.77 & 0.5447 \\
\hline 5 & 693 & 210.34 & 26.98 & 0.5532 \\
\hline 6 & 685 & 263.31 & 29.58 & 0.5659 \\
\hline 7 & 694 & 251.55 & 29.76 & 0.5540 \\
\hline 8 & 656 & 185.38 & 23.05 & 0.5726 \\
\hline 9 & 713 & 257.90 & 29.60 & 0.5744 \\
\hline 10 & 686 & 242.51 & 28.35 & 0.5562 \\
\hline 11 & 714 & 213.31 & 25.96 & 0.5587 \\
\hline 12 & 710 & 191.68 & 24.53 & 0.5477 \\
\hline 13 & 657 & 140.58 & 19.86 & 0.5522 \\
\hline 14 & 725 & 244.18 & 28.08 & 0.5674 \\
\hline 15 & 662 & 305.81 & 32.55 & 0.5940 \\
\hline 16 & 729 & 287.82 & 32.99 & 0.5674 \\
\hline 17 & 656 & 194.56 & 23.51 & 0.5666 \\
\hline 18 & 710 & 228.50 & 26.41 & 0.5863 \\
\hline 19 & 686 & 317.11 & 33.61 & 0.5850 \\
\hline 20 & 714 & 253.30 & 29.52 & 0.5551 \\
\hline 21 & 685 & 223.07 & 25.51 & 0.5821 \\
\hline 22 & 684 & 222.01 & 26.30 & 0.5683 \\
\hline 23 & 641 & 213.23 & 25.61 & 0.5637 \\
\hline 24 & 736 & 219.67 & 26.83 & 0.5846 \\
\hline 25 & 728 & 299.11 & 33.93 & 0.5808 \\
\hline 26 & 633 & 204.31 & 24.60 & 0.5645 \\
\hline 27 & 688 & 219.04 & 26.28 & 0.5698 \\
\hline 28 & 637 & 232.86 & 26.98 & 0.5646 \\
\hline 29 & 750 & 291.99 & 31.90 & 0.6148 \\
\hline 30 & 708 & 200.49 & 25.65 & 0.5592 \\
\hline 31 & 650 & 178.46 & 22.29 & 0.5484 \\
\hline 32 & 668 & 199.90 & 24.01 & 0.5639 \\
\hline 33 & 678 & 232.66 & 27.60 & 0.5724 \\
\hline 34 & 695 & 240.11 & 29.25 & 0.5546 \\
\hline 35 & 687 & 177.45 & 22.91 & 0.5573 \\
\hline 36 & 704 & 257.63 & 29.61 & 0.5685 \\
\hline 37 & 568 & 177.78 & 22.23 & 0.5469 \\
\hline 38 & 629 & 249.07 & 27.35 & 0.5752 \\
\hline 39 & 651 & 190.39 & 24.30 & 0.5591 \\
\hline 40 & 656 & 190.77 & 23.17 & 0.5591 \\
\hline 41 & 701 & 224.13 & 27.74 & 0.5446 \\
\hline 42 & 711 & 173.17 & 22.90 & 0.5581 \\
\hline
\end{tabular}




\begin{tabular}{|c|c|c|c|c|}
\hline PARCELA & $\mathbf{N} \geq \mathbf{1 0} \mathbf{~ c m}$ & $\begin{array}{c}\text { Biomasa } \\
(\mathbf{M g} / \mathbf{h a})\end{array}$ & $\begin{array}{c}\text { área Basal } \\
\left(\mathbf{m}^{\mathbf{2}}\right)\end{array}$ & $\begin{array}{c}\text { Densidad_madera } \\
\left(\mathbf{g r} / \mathbf{c m}^{\mathbf{3}}\right)\end{array}$ \\
\hline 43 & 690 & 257.38 & 28.88 & 0.5749 \\
\hline 44 & 731 & 204.87 & 25.21 & 0.5583 \\
\hline 45 & 813 & 281.11 & 31.68 & 0.5804 \\
\hline 46 & 733 & 249.91 & 27.89 & 0.5754 \\
\hline 47 & 676 & 270.42 & 29.81 & 0.5654 \\
\hline 48 & 695 & 310.92 & 33.47 & 0.5789 \\
\hline 49 & 680 & 202.60 & 25.10 & 0.5522 \\
\hline 50 & 691 & 309.68 & 33.80 & 0.5728 \\
\hline
\end{tabular}




\section{ANEXO C}

\section{Modelo Digital de Superficie normalizado (nMDS) de cada subparcela}




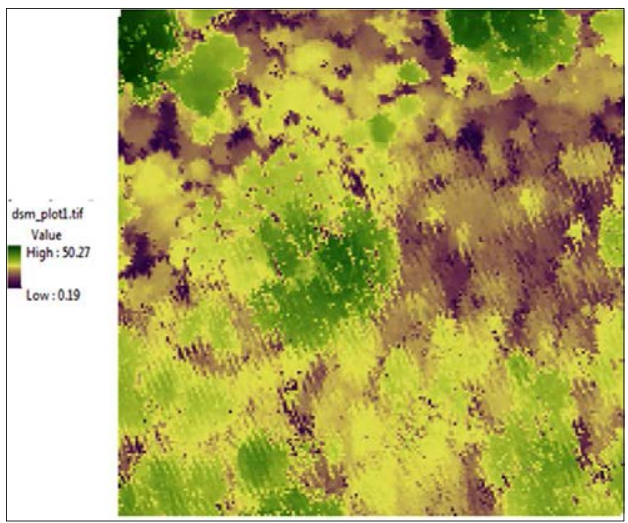

PARCELA 1

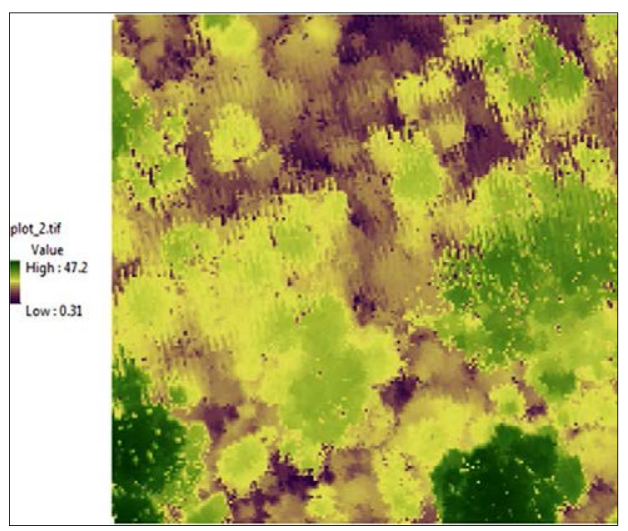

PARCELA 2

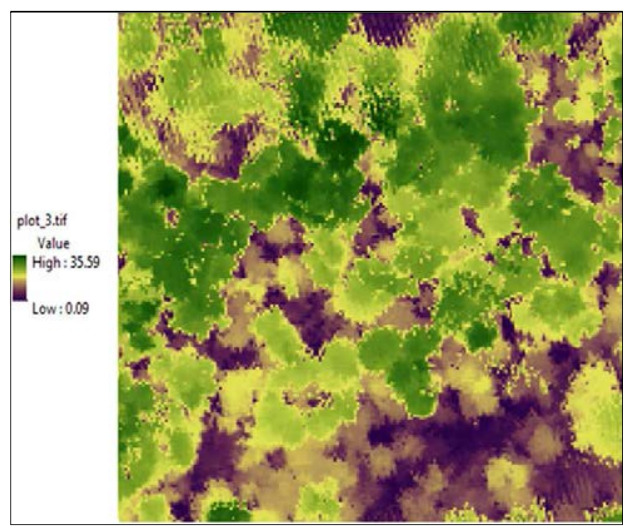

PARCELA 3

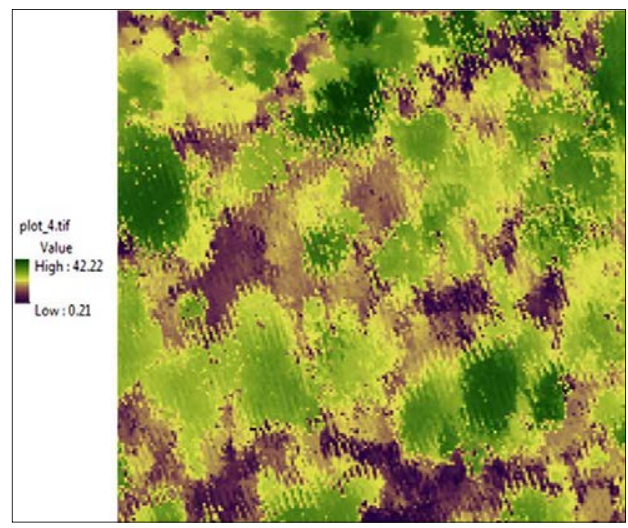

PARCELA 4

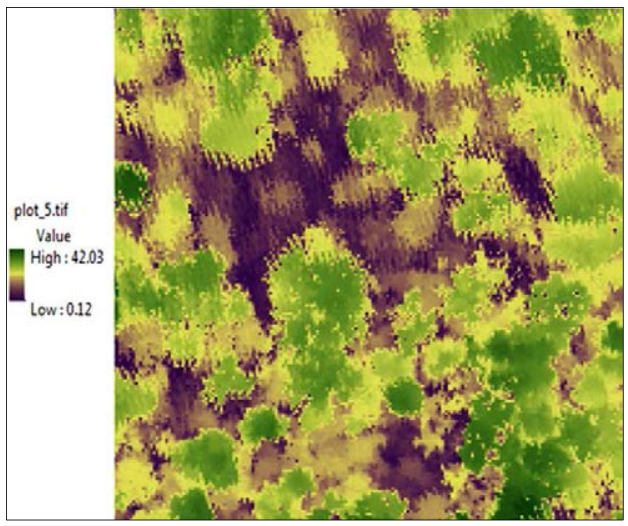

PARCELA 5

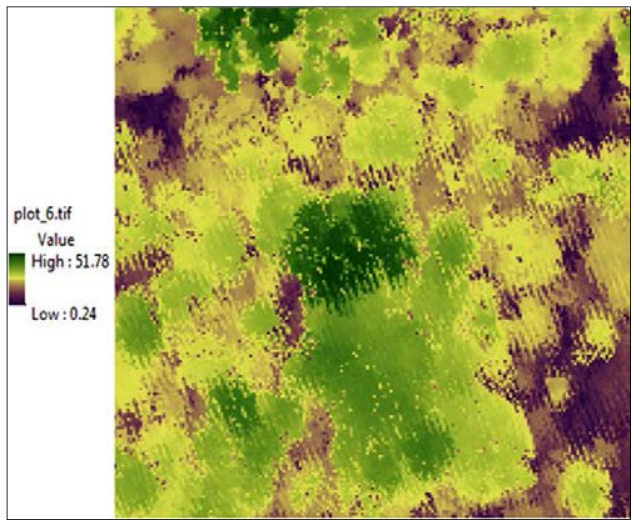

PARCELA 6

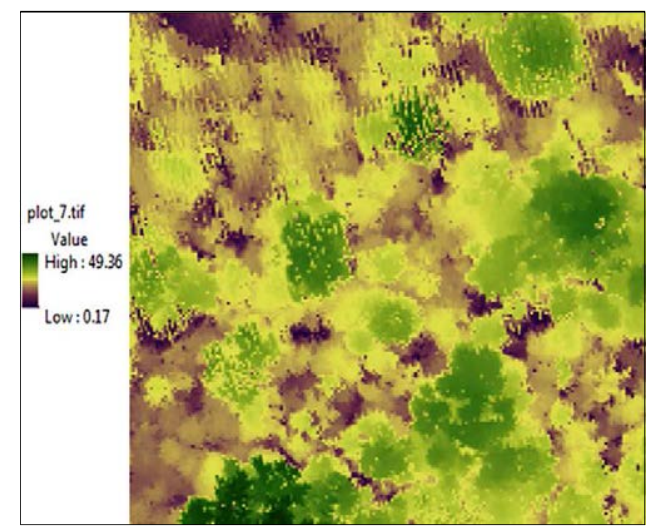

PARCELA 7

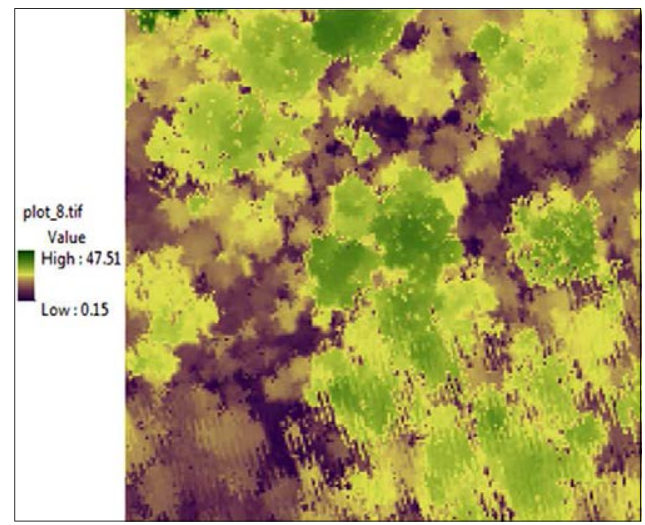

PARCELA 8 


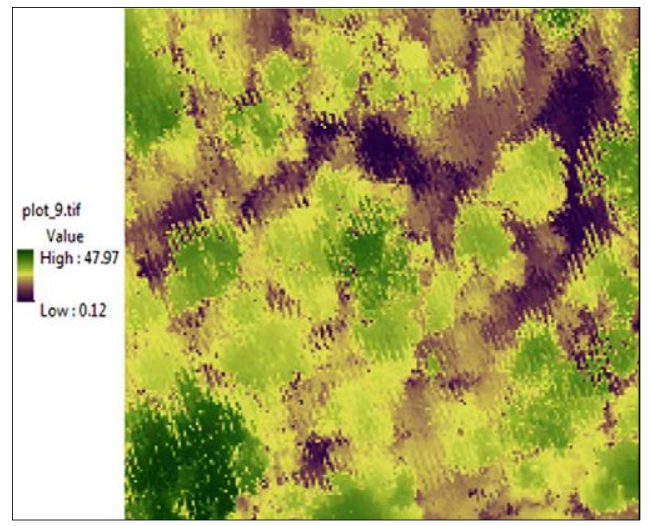

PARCELA 9

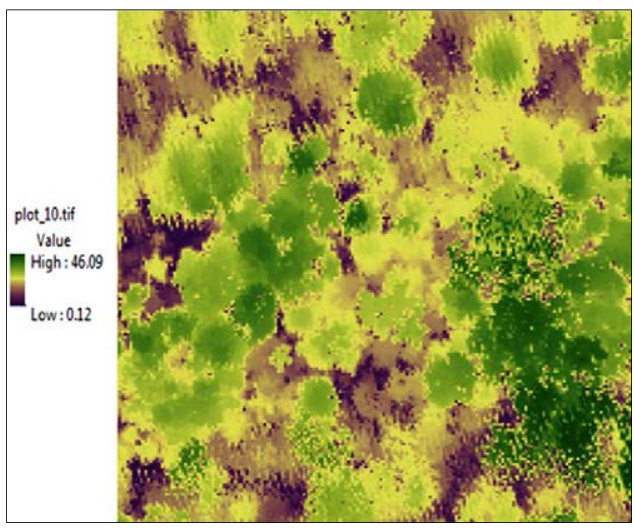

PARCELA 10

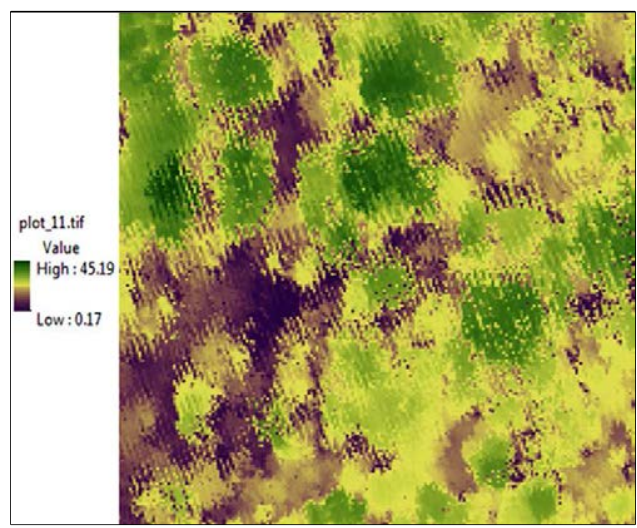

PARCELA 11

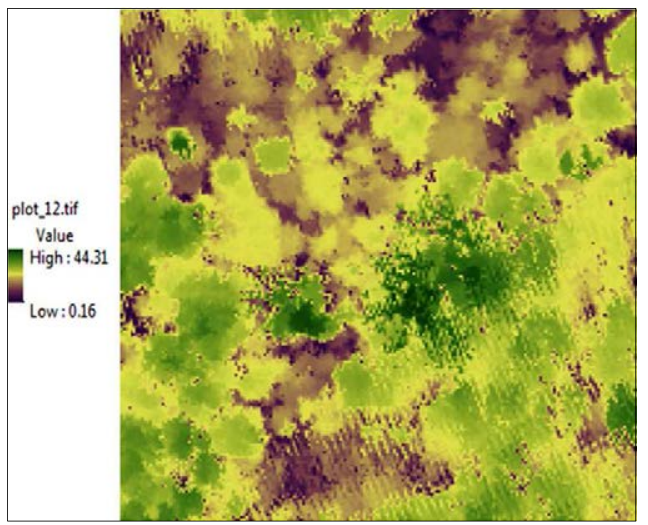

PARCELA 12

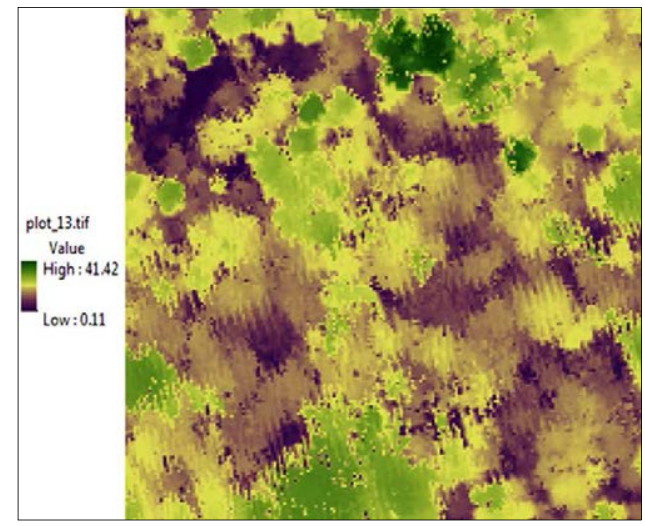

PARCELA 13

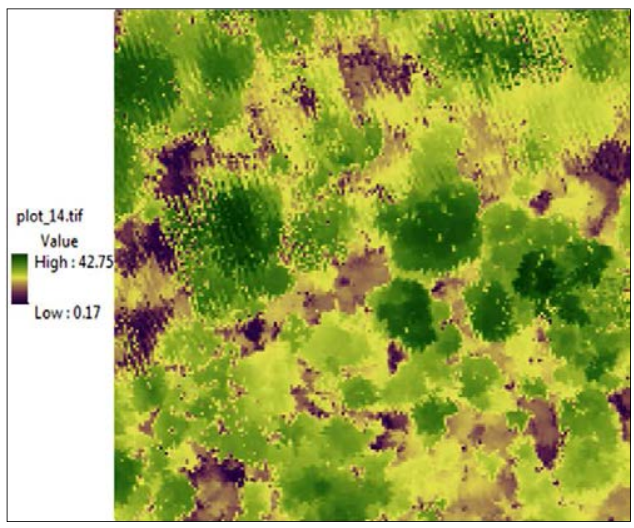

PARCELA 14

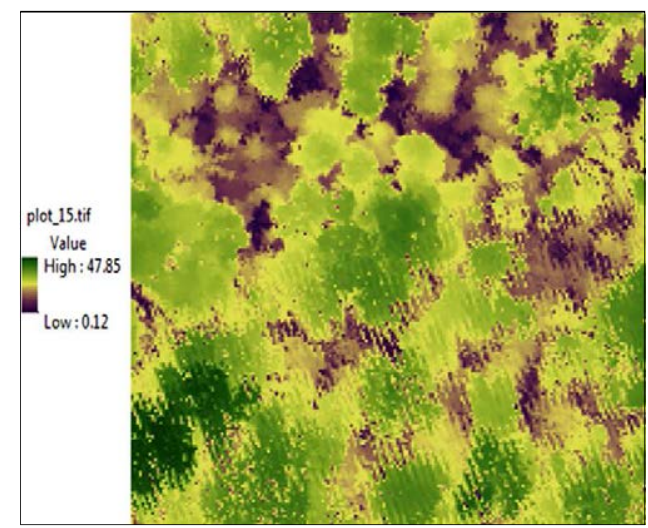

PARCELA 15

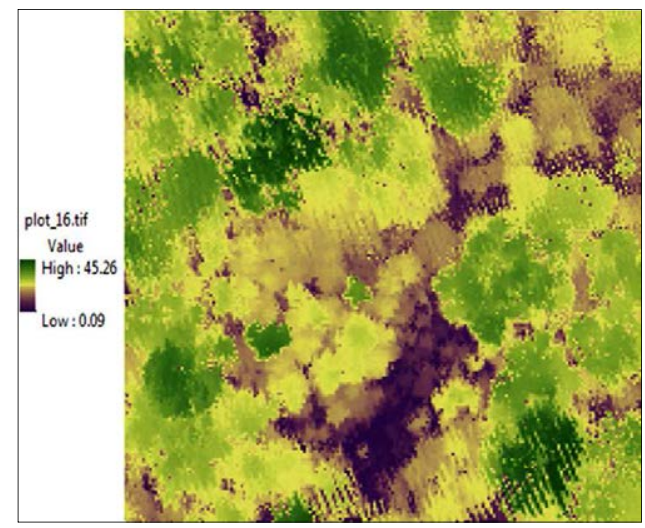

PARCELA 16 


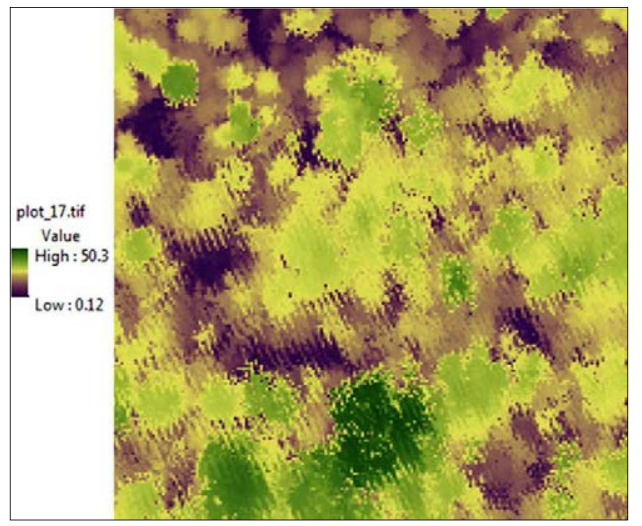

PARCELA 17

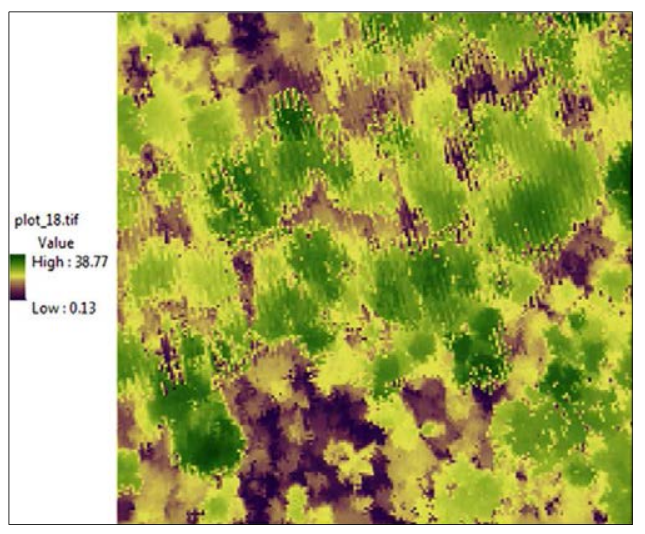

PARCELA 18

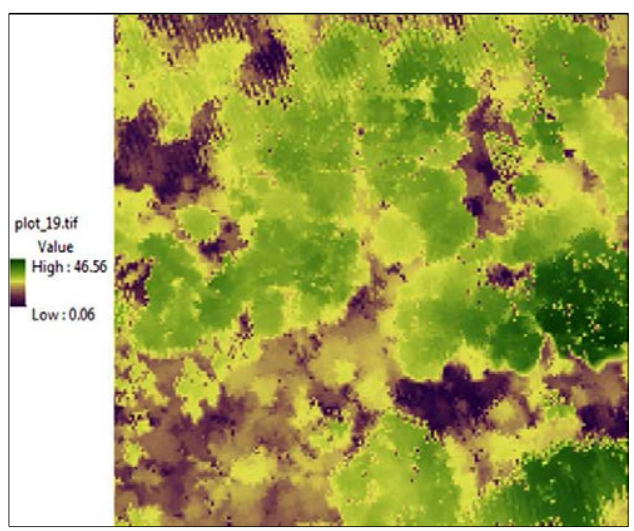

PARCELA19

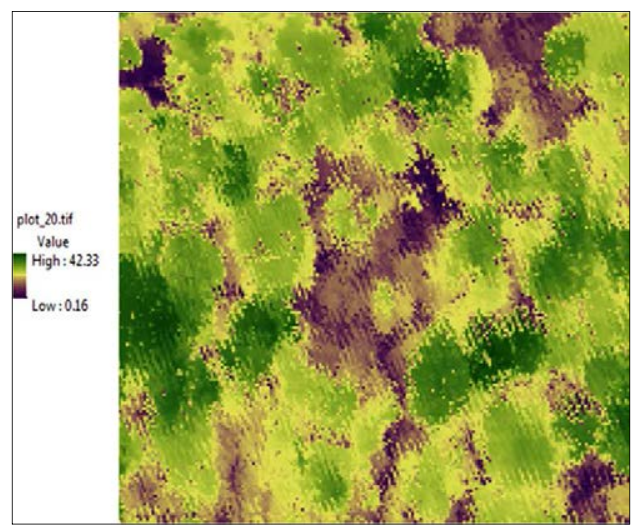

PARCELA 20

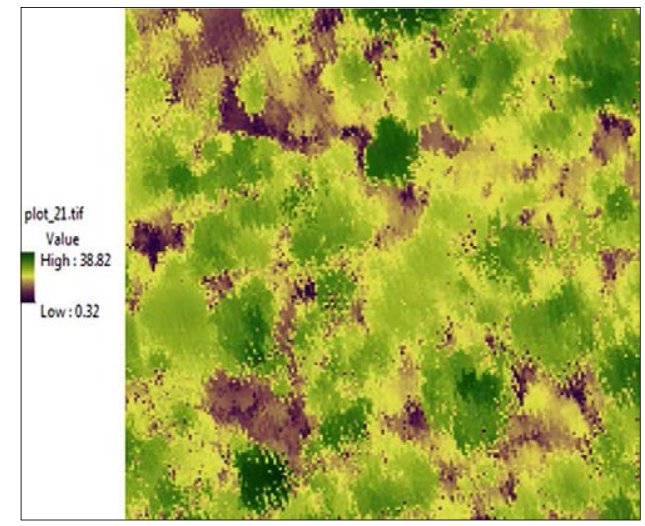

PARCELA 21

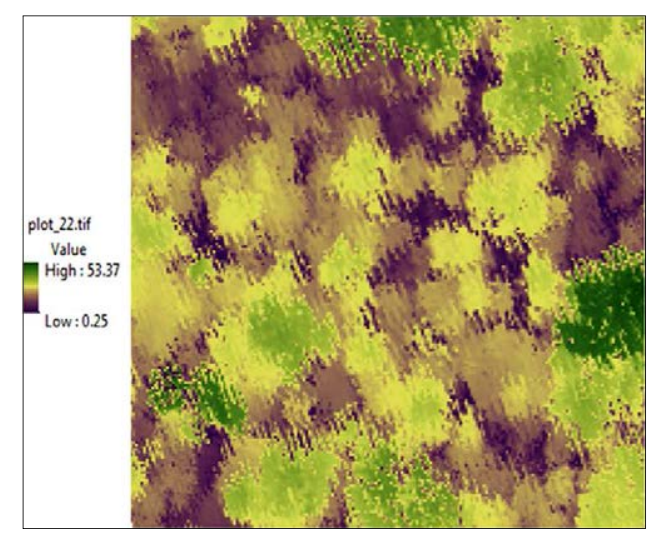

PARCELA 22

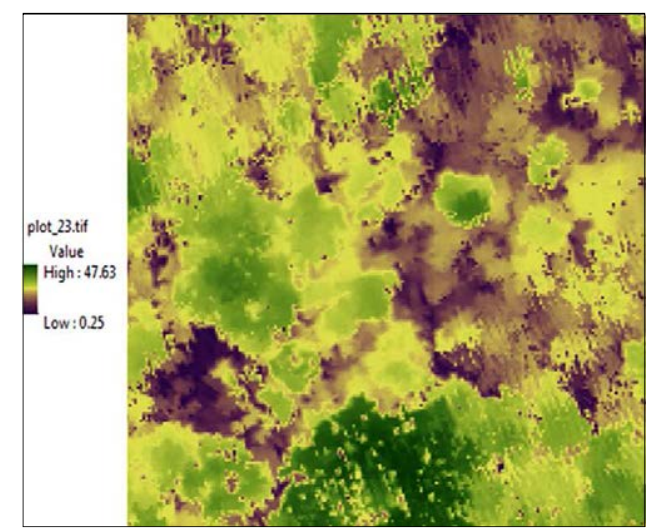

PARCELA 23

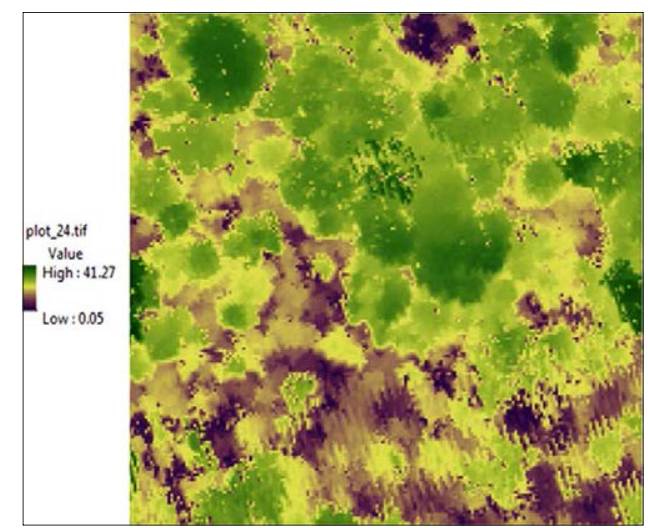

PARCELA 24 


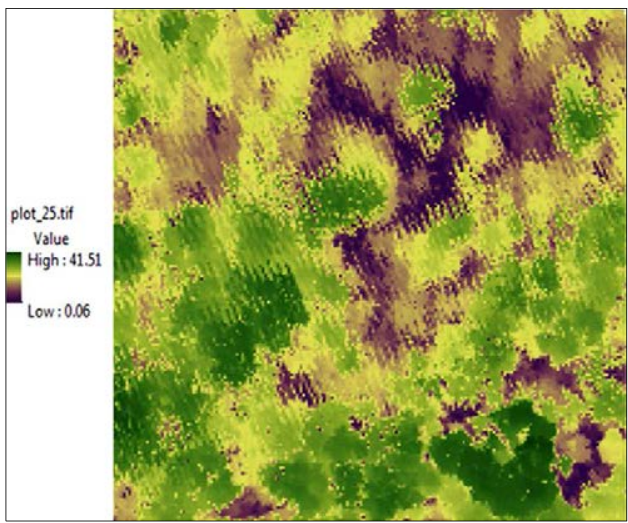

PARCELA 25

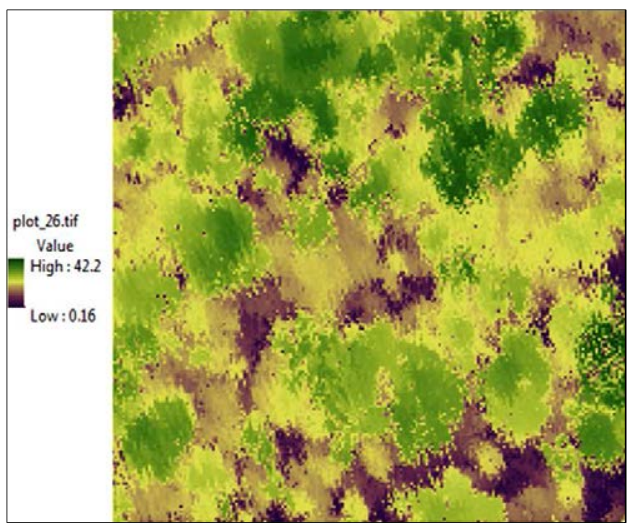

PARCELA 26

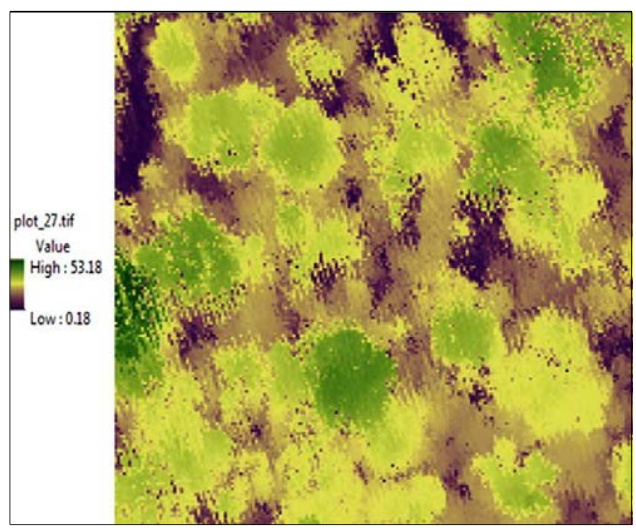

PARCELA 27

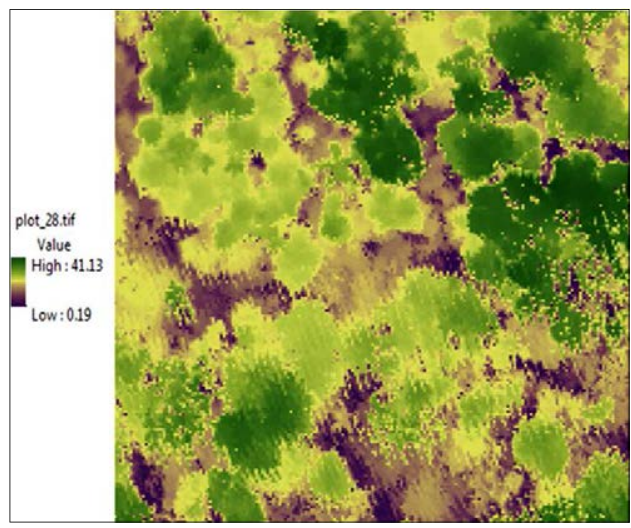

PARCELA 28

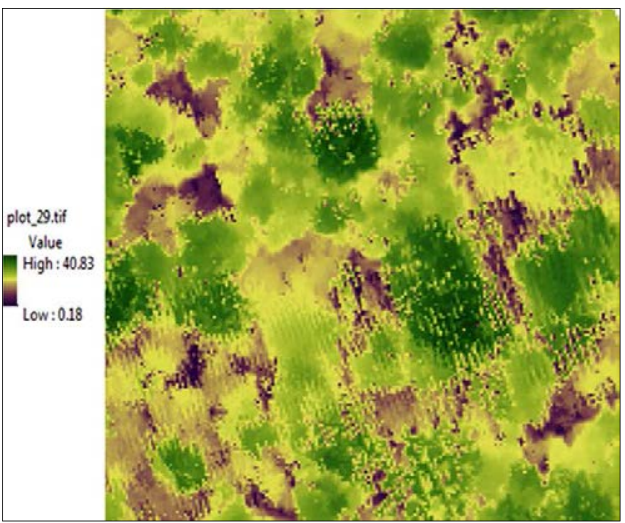

PARCELA 29

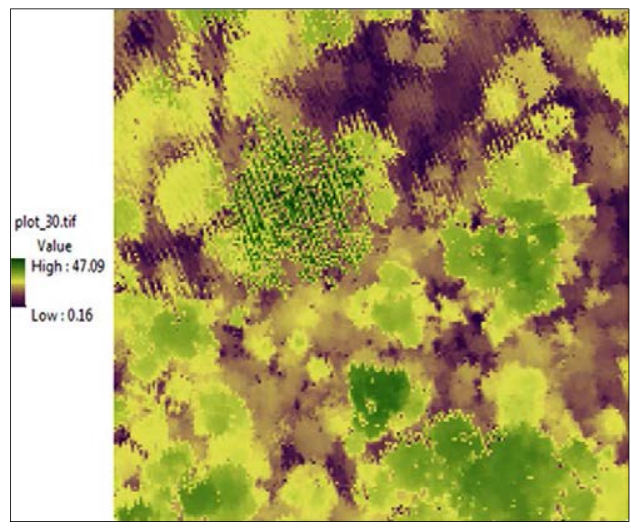

PARCELA 30

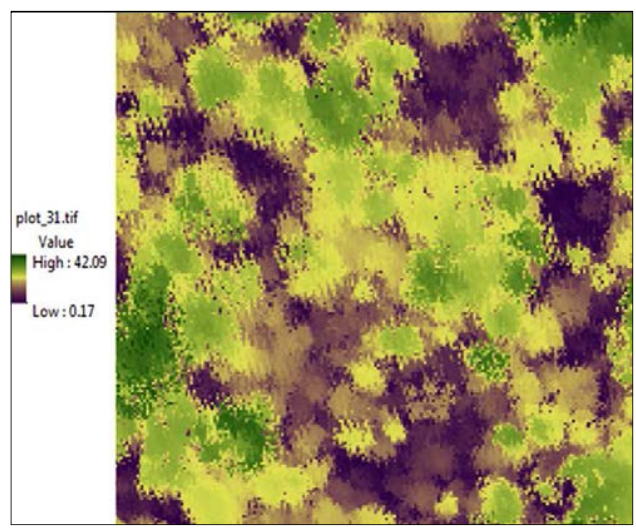

PARCELA 31

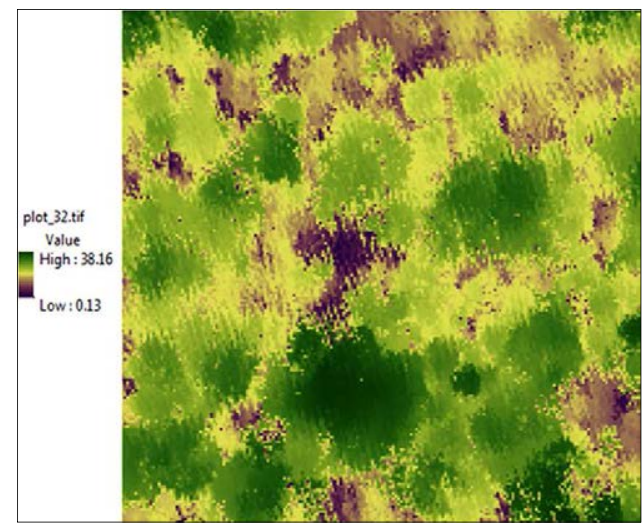

PARCELA 32 


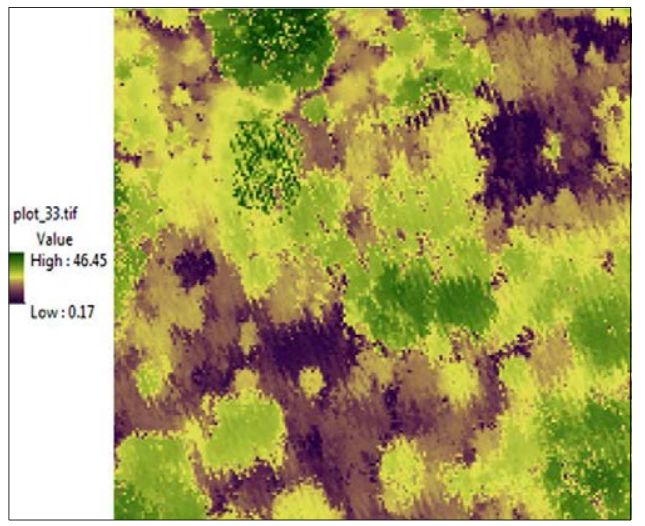

PARCELA 33

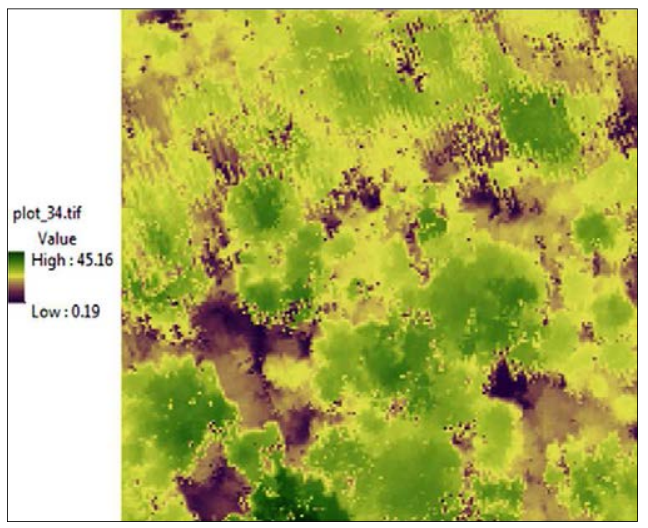

PARCELA 34

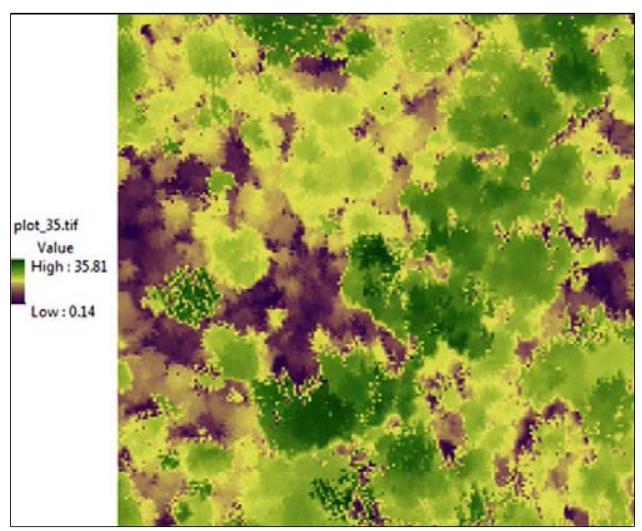

PARCELA 35

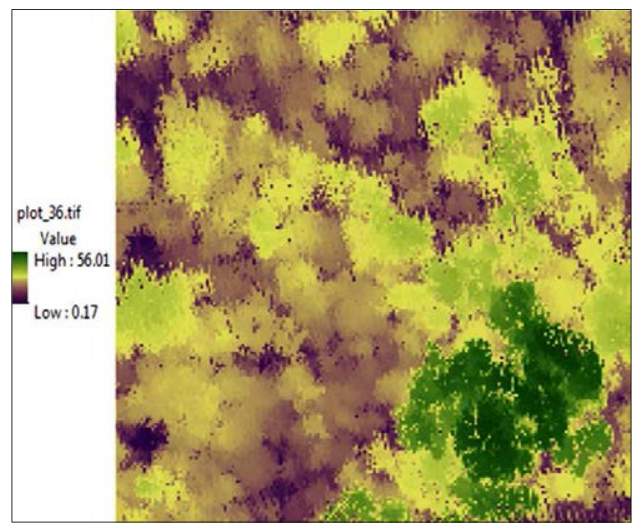

PARCELA 36

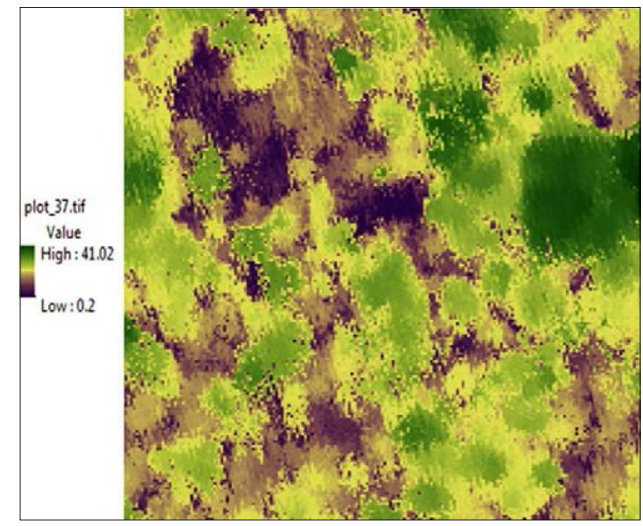

PARCELA 37

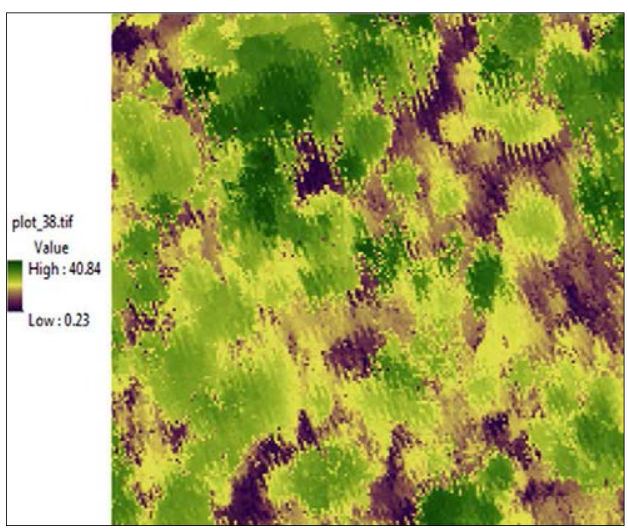

PARCELA 38

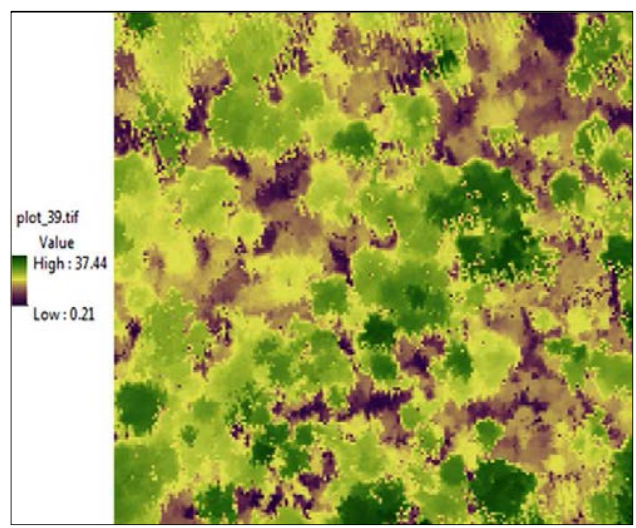

PARCELA 39

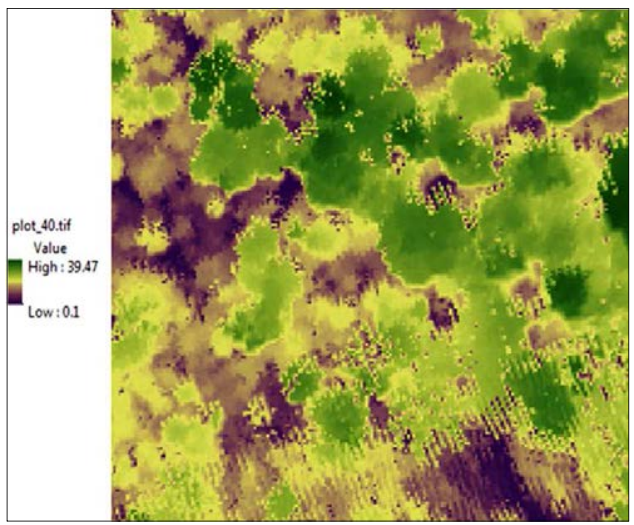

PARCELA 40 


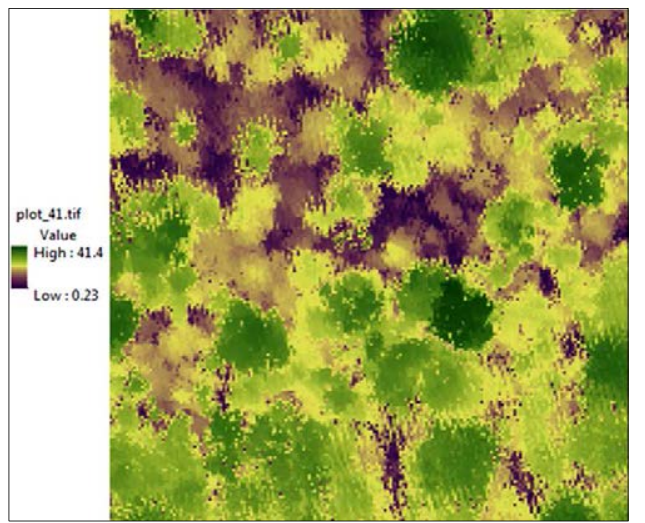

PARCELA 41

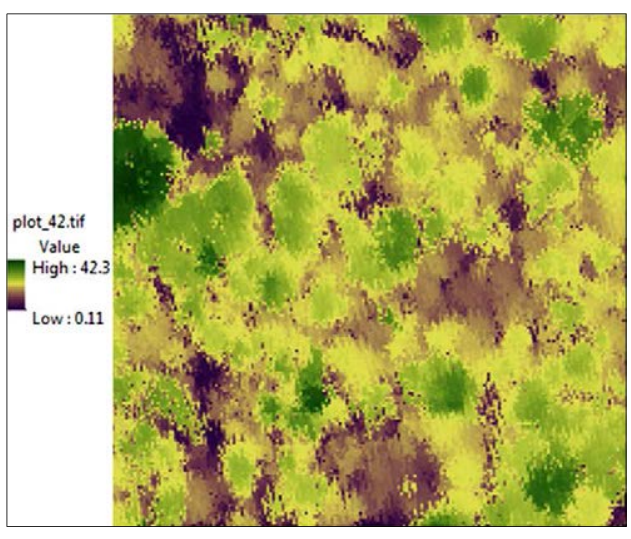

PARCELA 42

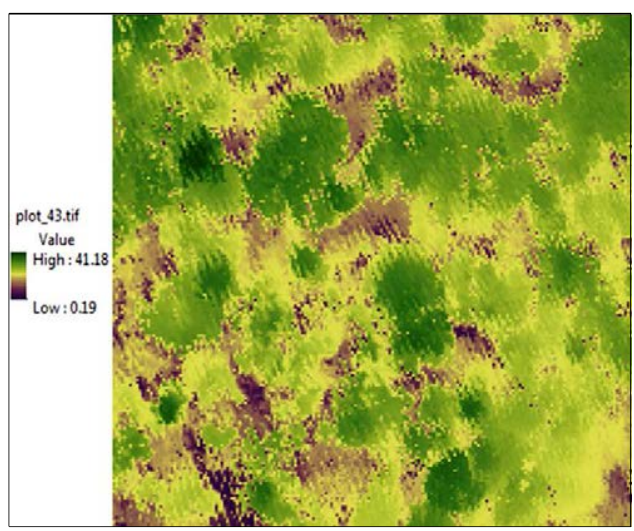

PARCELA 43

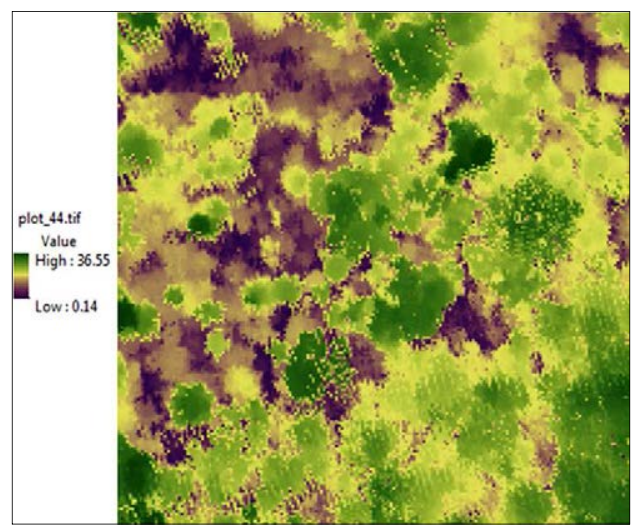

PARCELA 44

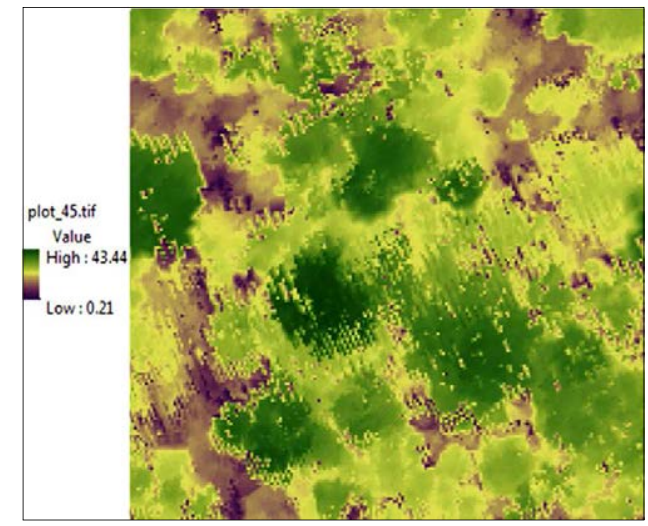

PARCELA 45

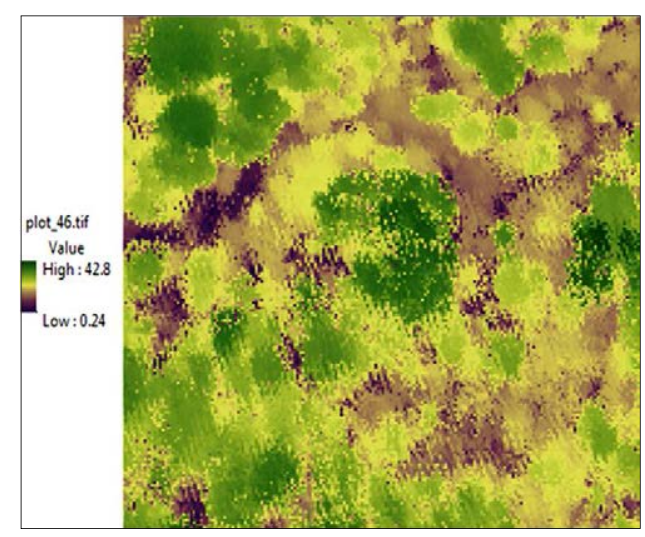

PARCELA 46

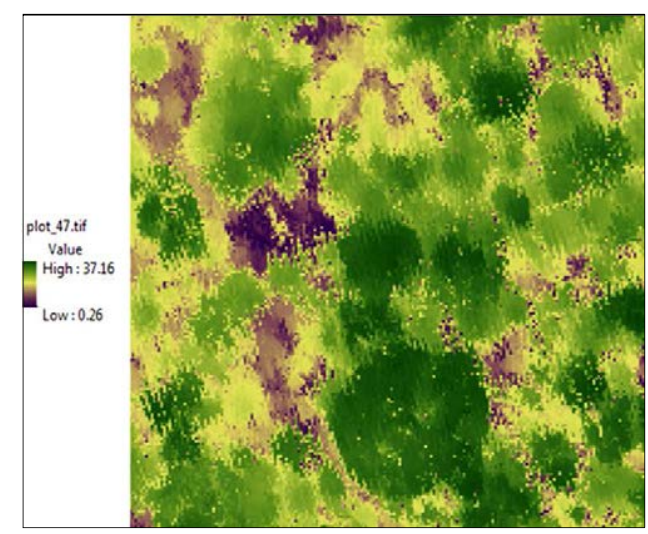

PARCELA 47

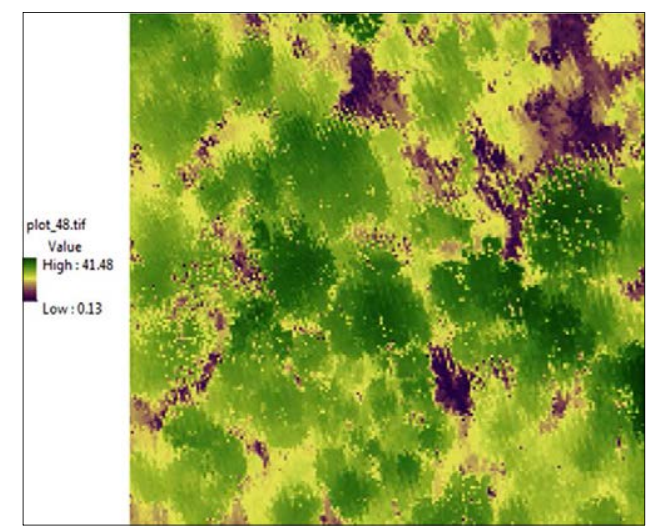

PARCELA 48 


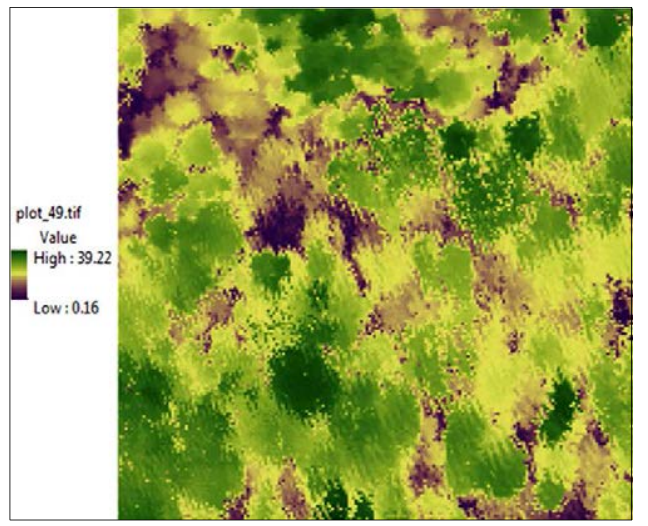

PARCELA 49

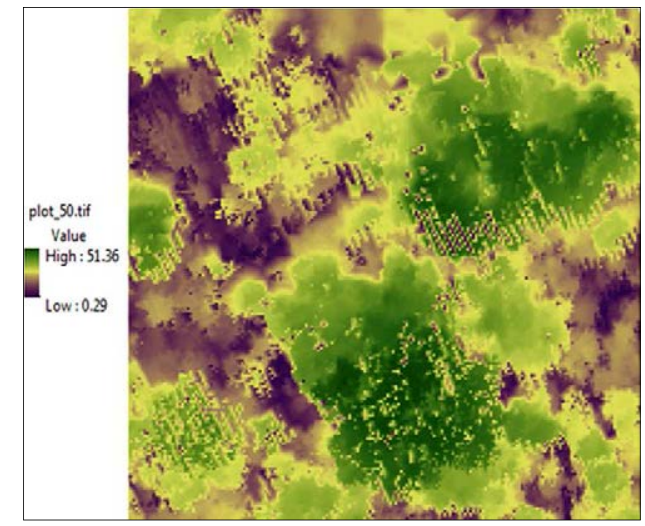

PARCELA 50 


\section{ANEXO D}

\section{Resumen de las estadísticas \\ descriptivas del $\mathrm{TCH}$ y $\mathrm{MCH}$ de las \\ subparcelas}




\begin{tabular}{|c|c|c|c|c|c|c|c|c|c|c|c|c|c|}
\hline PARCELA & TCH & STD & CV_TCH & MCH & STD & CV_MCH & PARCELA & TCH & STD & CV_TCH & MCH & STD & CV_MCH \\
\hline 1 & 23.23 & 8.19 & 0.35 & 22.36 & 8.82 & 0.39 & 26 & 21.58 & 7.51 & 0.35 & 20.85 & 8.31 & 0.40 \\
\hline 2 & 22.74 & 8.51 & 0.37 & 21.55 & 8.94 & 0.41 & 27 & 23.29 & 8.14 & 0.35 & 22.58 & 8.93 & 0.40 \\
\hline 3 & 18.12 & 8.19 & 0.45 & 17.50 & 8.63 & 0.49 & 28 & 22.37 & 8.55 & 0.38 & 21.38 & 9.28 & 0.43 \\
\hline 4 & 21.58 & 8.59 & 0.40 & 20.68 & 9.49 & 0.46 & 29 & 22.40 & 6.94 & 0.31 & 21.48 & 8.16 & 0.38 \\
\hline 5 & 19.39 & 8.54 & 0.44 & 18.19 & 9.16 & 0.50 & 30 & 20.26 & 8.32 & 0.41 & 19.04 & 9.26 & 0.49 \\
\hline 6 & 25.29 & 9.32 & 0.37 & 24.15 & 10.29 & 0.43 & 31 & 17.47 & 8.41 & 0.48 & 17.20 & 9.08 & 0.53 \\
\hline 7 & 23.90 & 7.98 & 0.33 & 22.72 & 8.74 & 0.38 & 32 & 22.62 & 7.05 & 0.31 & 21.77 & 7.87 & 0.36 \\
\hline 8 & 20.12 & 7.83 & 0.39 & 19.53 & 8.42 & 0.43 & 33 & 20.78 & 8.79 & 0.42 & 19.80 & 9.35 & 0.47 \\
\hline 9 & 22.44 & 9.02 & 0.40 & 21.49 & 9.80 & 0.46 & 34 & 23.09 & 6.98 & 0.30 & 21.99 & 7.92 & 0.36 \\
\hline 10 & 23.53 & 8.48 & 0.36 & 21.94 & 9.58 & 0.44 & 35 & 18.88 & 7.08 & 0.38 & 18.28 & 7.81 & 0.43 \\
\hline 11 & 21.46 & 8.66 & 0.40 & 20.16 & 9.71 & 0.48 & 36 & 23.89 & 10.35 & 0.43 & 22.78 & 10.83 & 0.48 \\
\hline 12 & 21.34 & 7.31 & 0.34 & 20.97 & 8.00 & 0.38 & 37 & 19.87 & 7.90 & 0.40 & 18.89 & 8.54 & 0.45 \\
\hline 13 & 16.96 & 6.97 & 0.41 & 16.37 & 7.43 & 0.45 & 38 & 21.58 & 8.03 & 0.37 & 20.63 & 8.99 & 0.44 \\
\hline 14 & 24.22 & 7.92 & 0.33 & 23.10 & 9.15 & 0.40 & 39 & 19.14 & 6.99 & 0.37 & 18.18 & 7.89 & 0.43 \\
\hline 15 & 24.39 & 8.70 & 0.36 & 23.98 & 9.71 & 0.41 & 40 & 20.04 & 7.95 & 0.40 & 18.96 & 8.61 & 0.45 \\
\hline 16 & 22.86 & 8.56 & 0.37 & 22.26 & 9.13 & 0.41 & 41 & 21.10 & 8.22 & 0.39 & 20.54 & 8.83 & 0.43 \\
\hline 17 & 21.78 & 8.70 & 0.40 & 21.45 & 9.42 & 0.44 & 42 & 19.48 & 7.00 & 0.36 & 19.04 & 7.49 & 0.39 \\
\hline 18 & 19.71 & 7.46 & 0.38 & 19.61 & 8.13 & 0.41 & 43 & 23.40 & 6.34 & 0.27 & 22.45 & 7.35 & 0.33 \\
\hline 19 & 23.57 & 8.75 & 0.37 & 22.99 & 9.61 & 0.42 & 44 & 18.64 & 7.62 & 0.41 & 18.48 & 8.13 & 0.44 \\
\hline 20 & 22.63 & 8.08 & 0.36 & 21.86 & 8.91 & 0.41 & 45 & 24.31 & 7.74 & 0.32 & 23.64 & 8.85 & 0.37 \\
\hline 21 & 21.13 & 6.12 & 0.29 & 20.39 & 6.97 & 0.34 & 46 & 22.37 & 7.39 & 0.33 & 21.73 & 8.28 & 0.38 \\
\hline 22 & 22.04 & 9.19 & 0.42 & 21.71 & 10.09 & 0.46 & 47 & 23.84 & 7.55 & 0.32 & 23.10 & 8.47 & 0.37 \\
\hline 23 & 22.53 & 8.72 & 0.39 & 22.12 & 9.28 & 0.42 & 48 & 25.18 & 8.01 & 0.32 & 24.36 & 8.96 & 0.37 \\
\hline 24 & 21.46 & 7.68 & 0.36 & 20.26 & 8.41 & 0.42 & 49 & 22.18 & 7.36 & 0.33 & 21.75 & 8.10 & 0.37 \\
\hline 25 & 21.47 & 8.73 & 0.41 & 20.17 & 9.34 & 0.46 & 50 & 26.13 & 11.04 & 0.42 & 25.06 & 12.19 & 0.49 \\
\hline
\end{tabular}

$\mathbf{T C H}=$ altura media al tope del dosel; $\mathbf{M C H}=$ altura media del perfil de dosel; $\mathbf{S T D =}$ desviación estándar; $\mathbf{C V =}$ coeficiente de variación . 


\section{ANEXO E}

\section{Análisis exploratorio de los datos \\ LiDAR de las subparcelas de estudio}




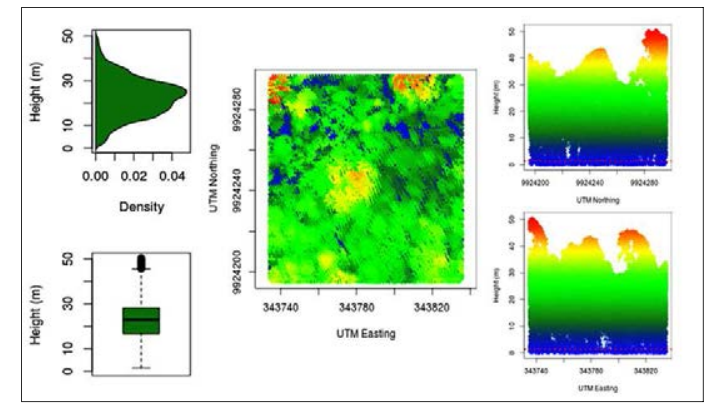

PARCELA 1

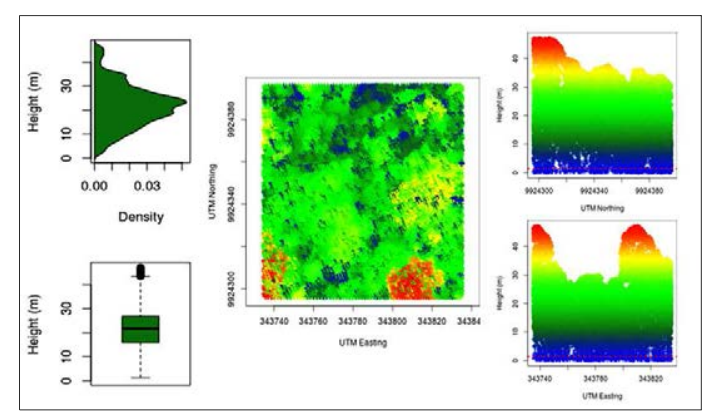

PARCELA 2

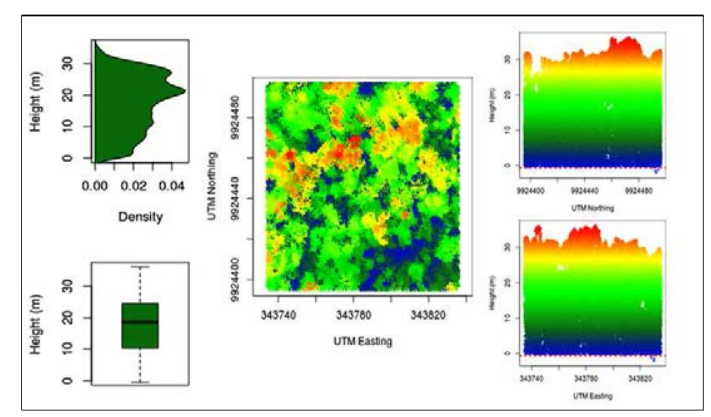

PARCELA 3

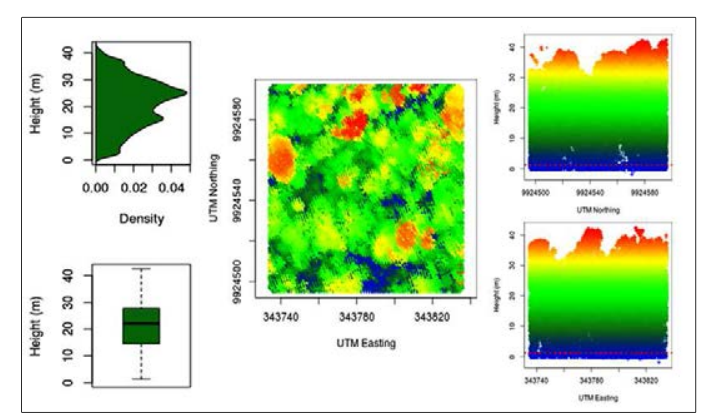

PARCELA 4

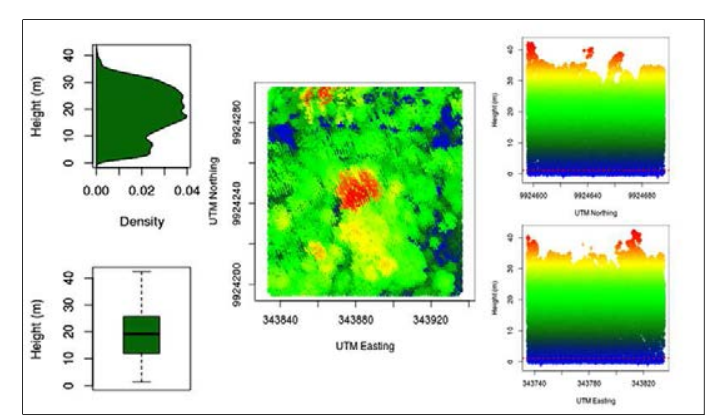

PARCELA 5

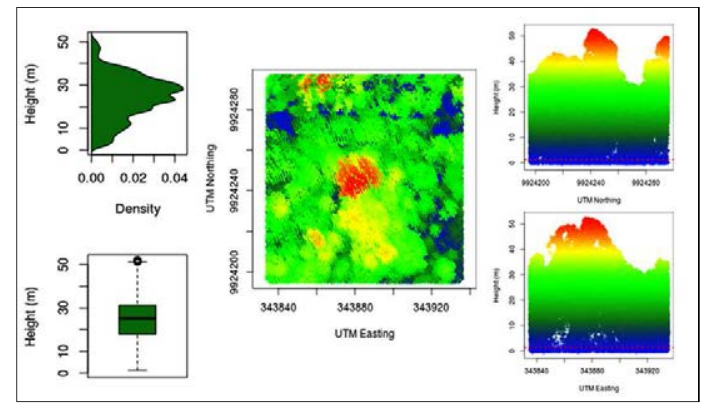

PARCELA 6

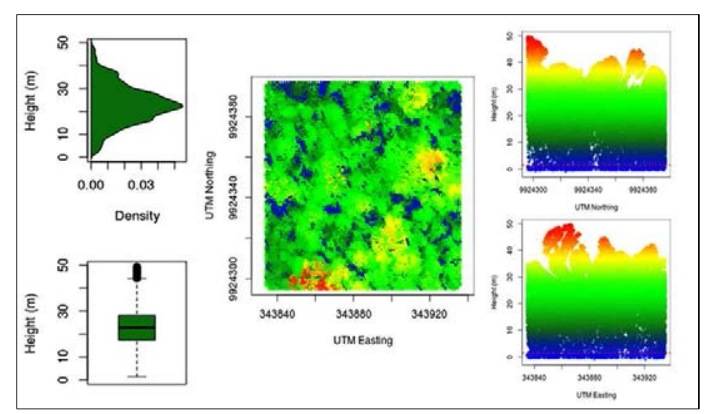

PARCELA 7

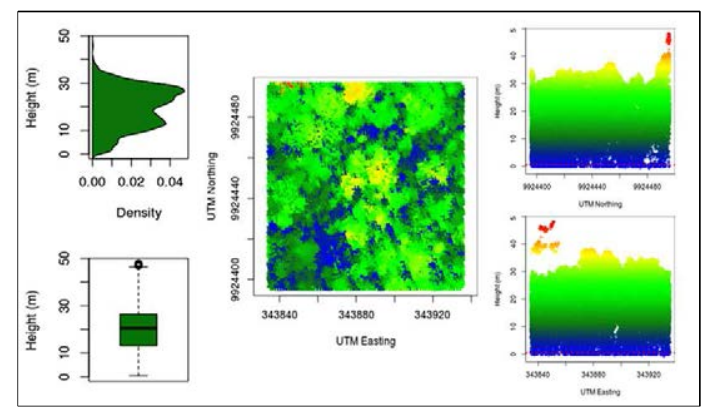

PARCELA 8

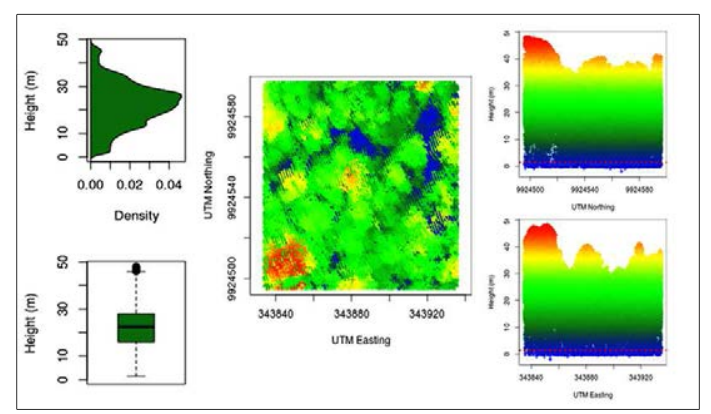

PARCELA 9

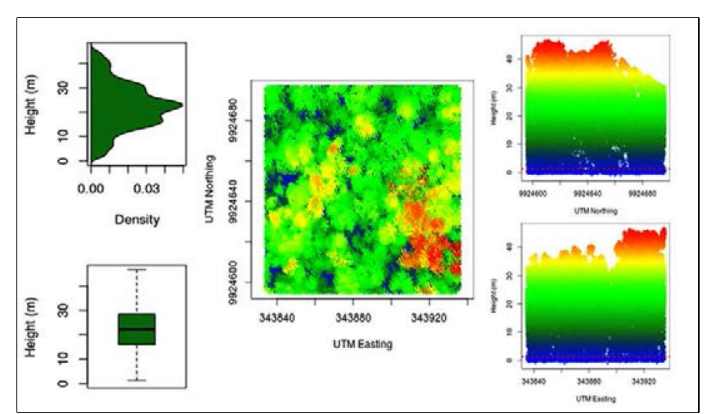

PARCELA 10 


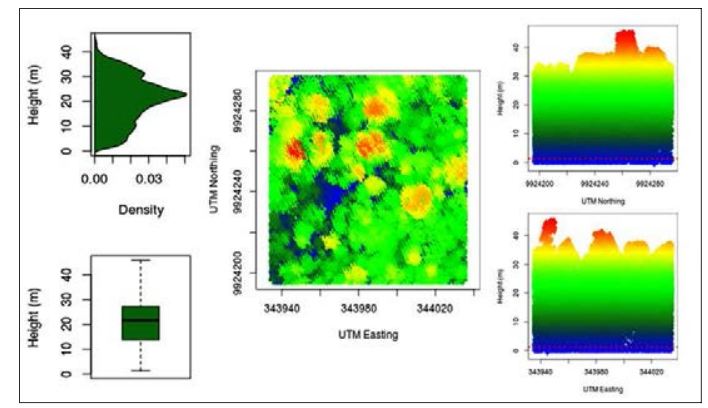

PARCELA 11

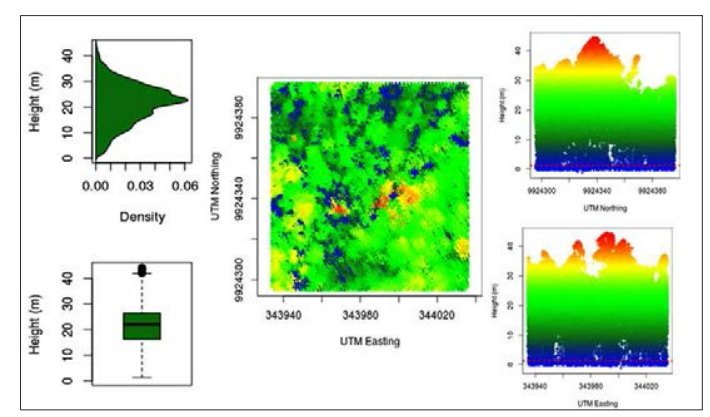

PARCELA 12

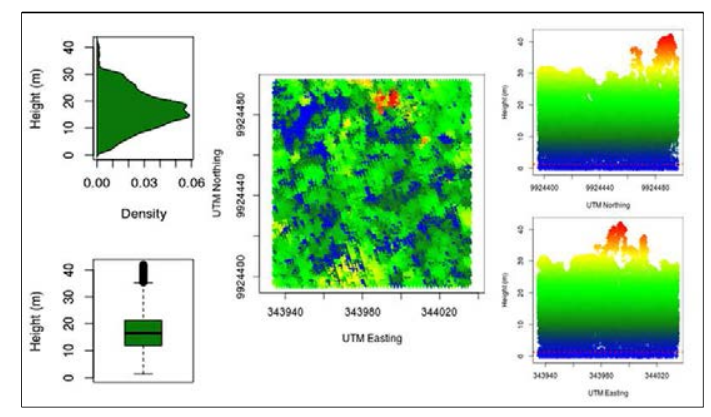

PARCELA 13

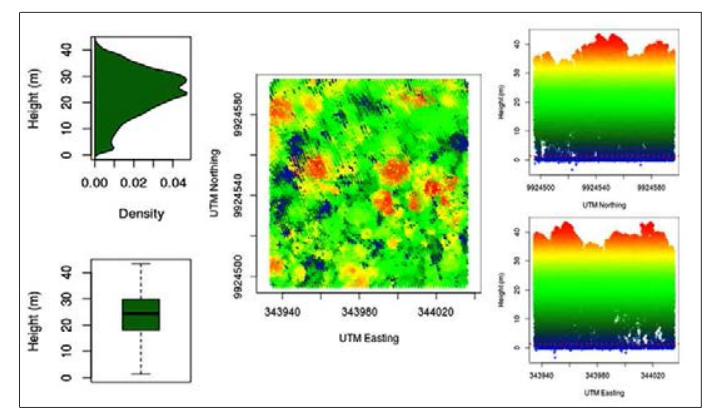

PARCELA 14

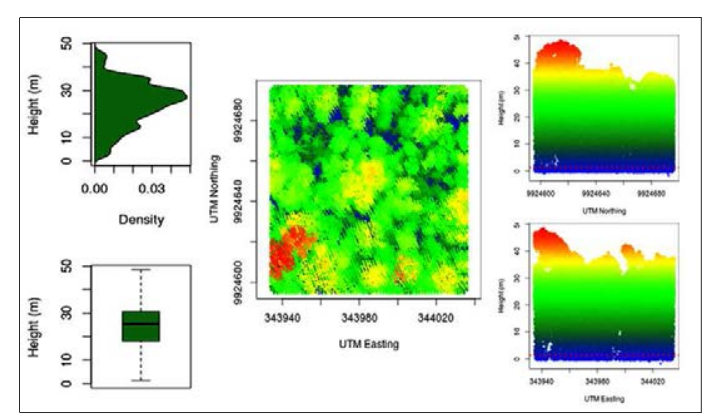

PARCELA 15

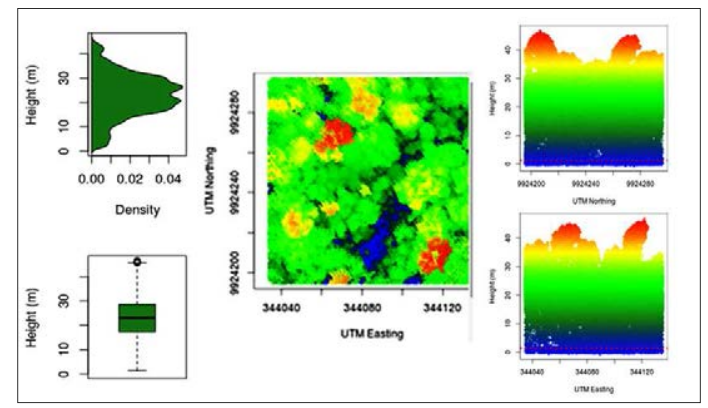

PARCELA 16

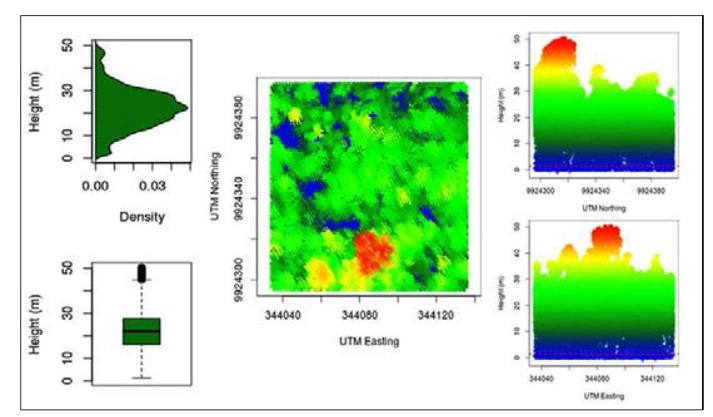

PARCELA 17

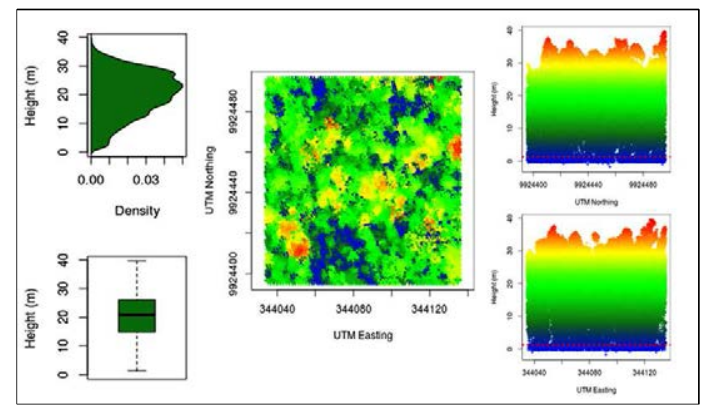

PARCELA 18

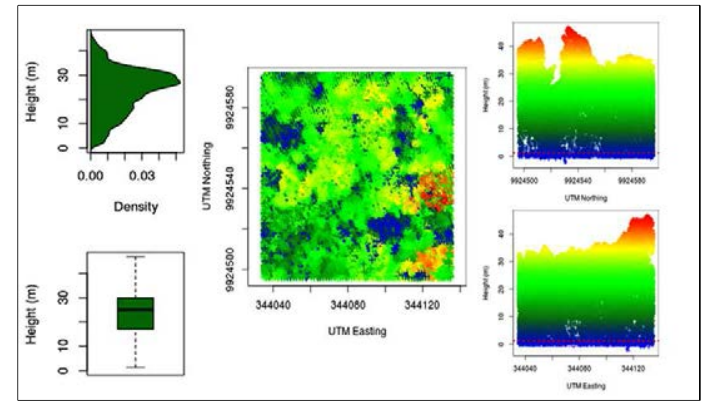

PARCELA 19

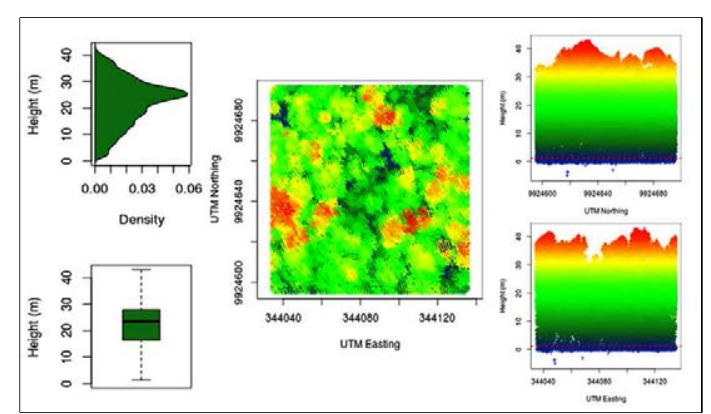

PARCELA 20 


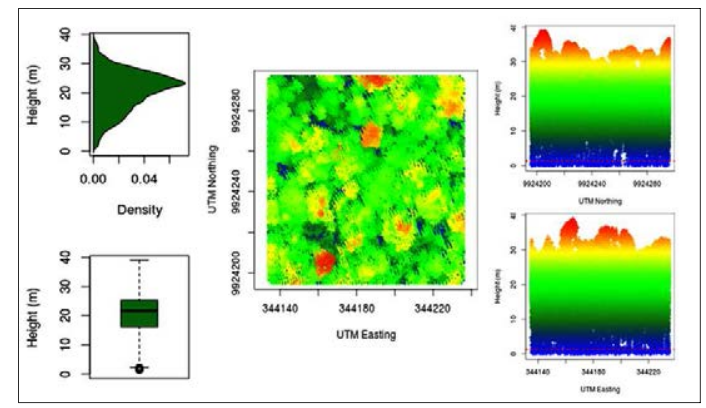

PARCELA 21

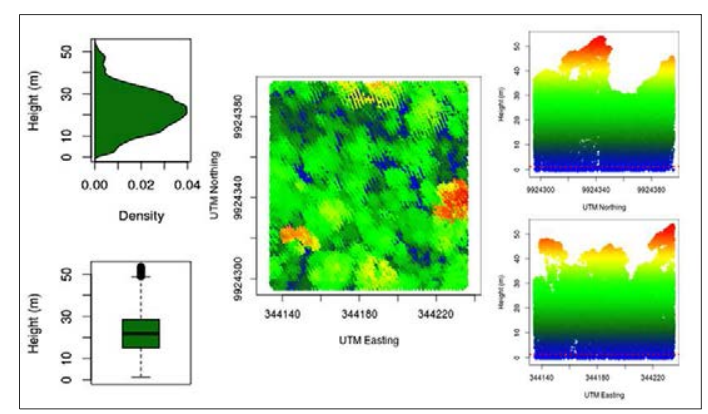

PARCELA 22

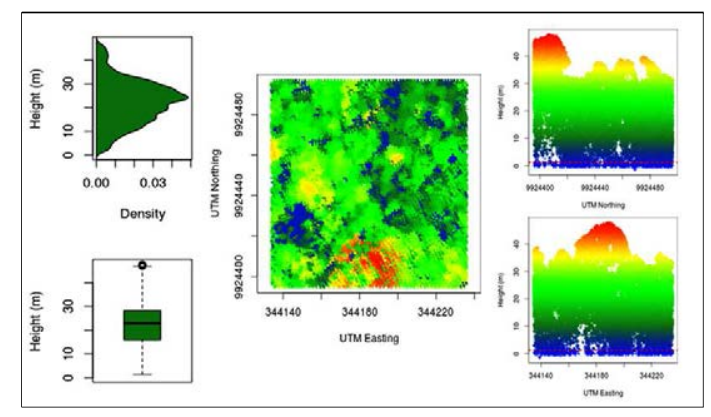

PARCELA 23

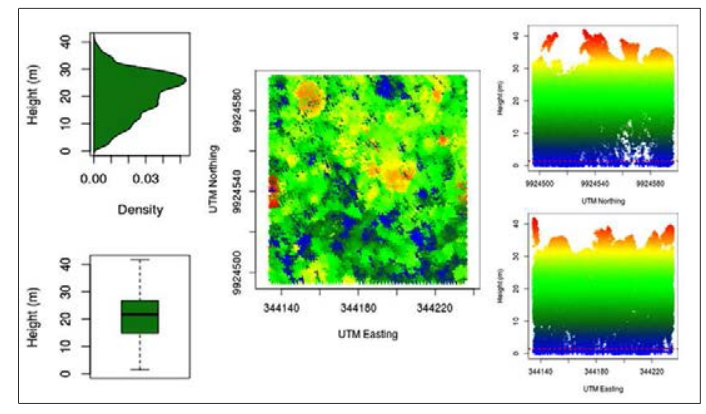

PARCELA 24

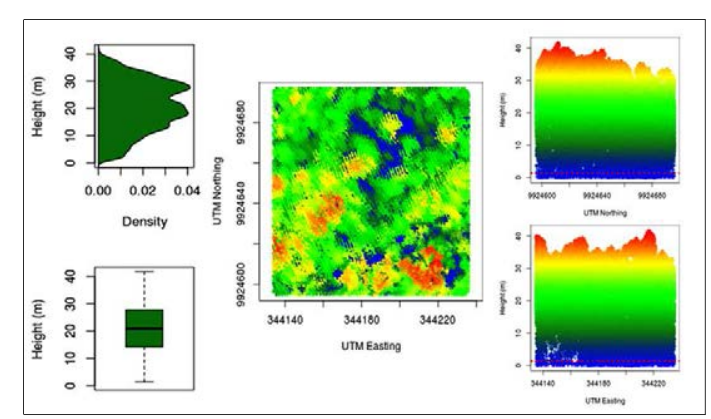

PARCELA 25

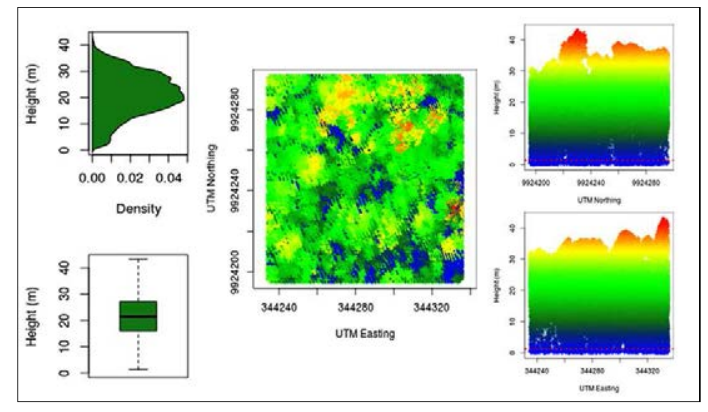

PARCELA 26

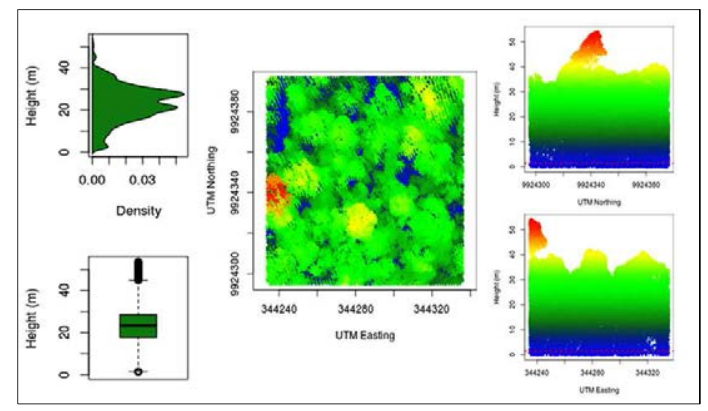

PARCELA 27

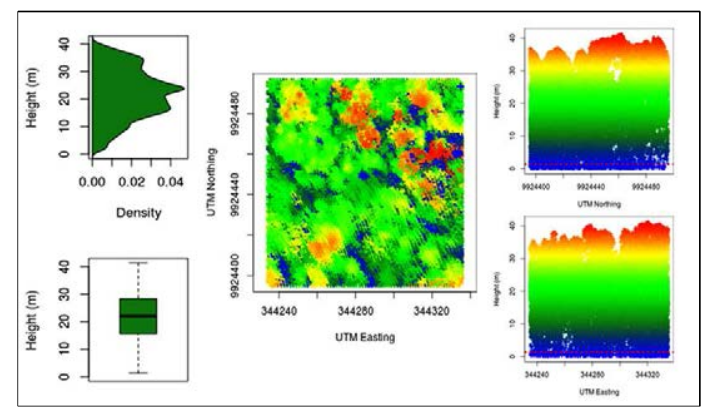

PARCELA 28

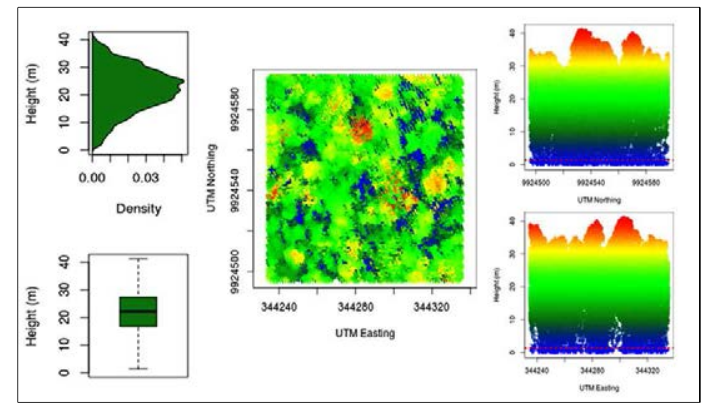

PARCELA 29

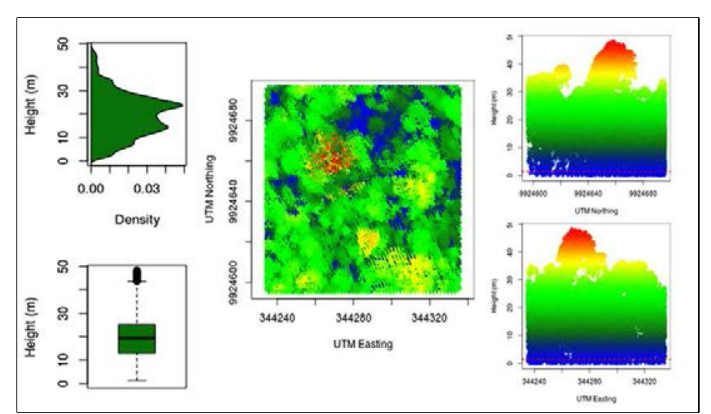

PARCELA 30 


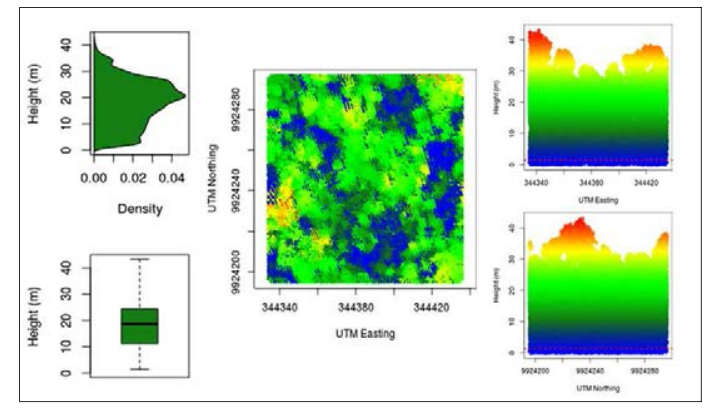

PARCELA 31

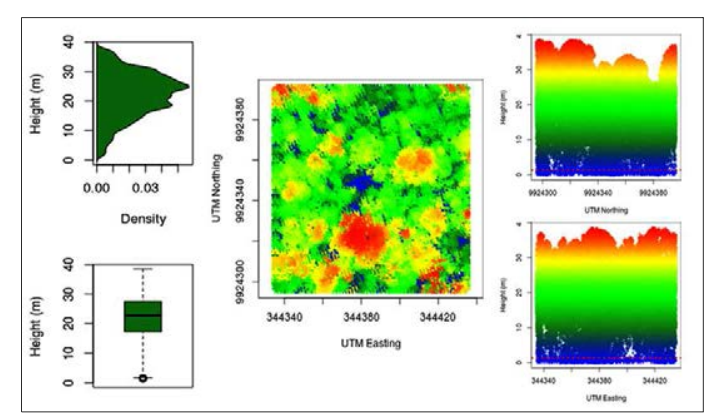

PARCELA 32

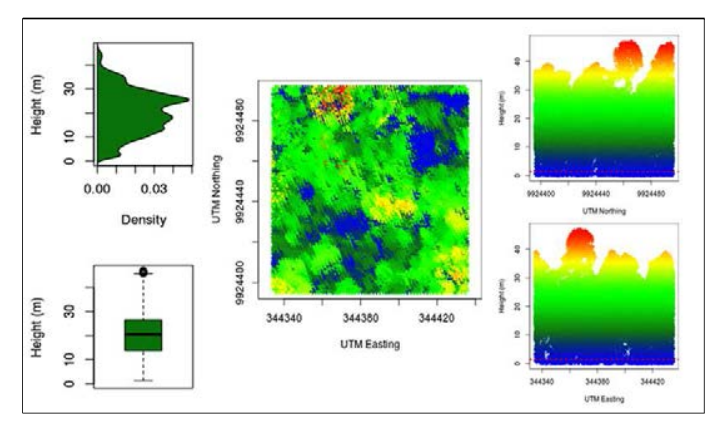

PARCELA 33

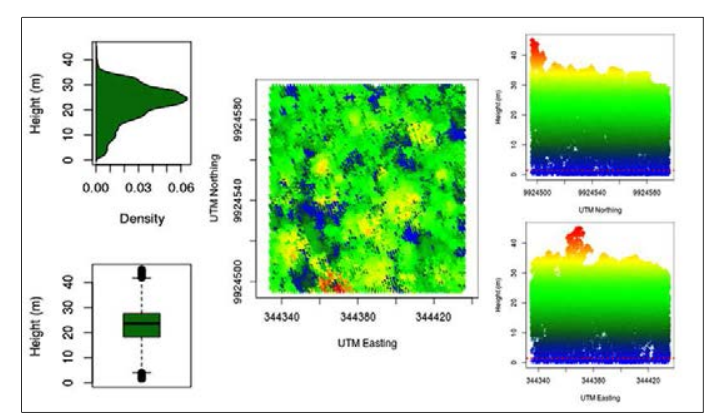

PARCELA 34

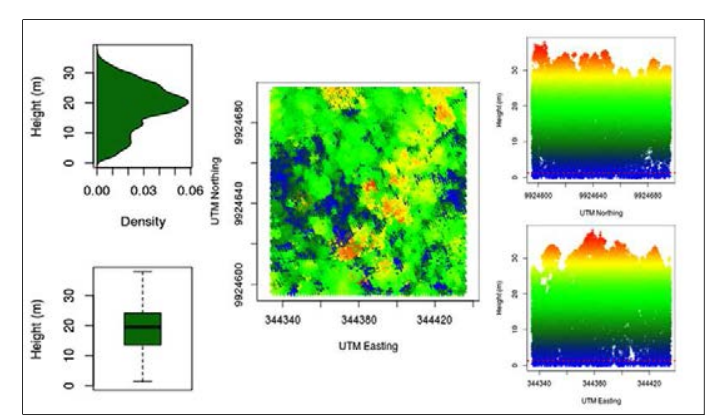

PARCELA 35

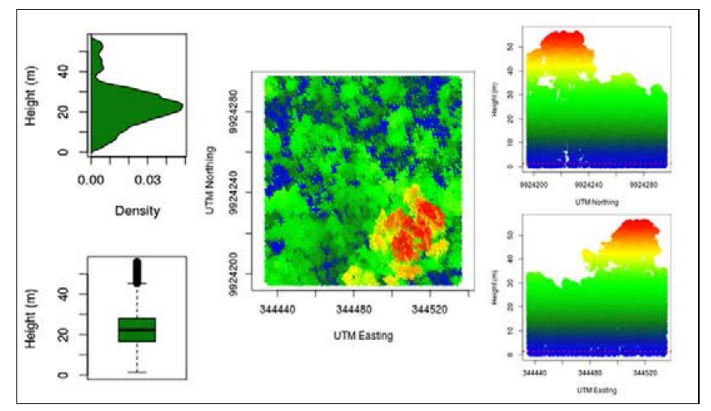

PARCELA 36

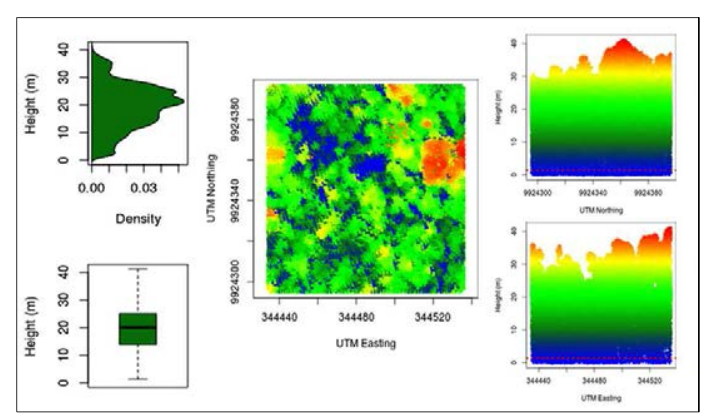

PARCELA 37

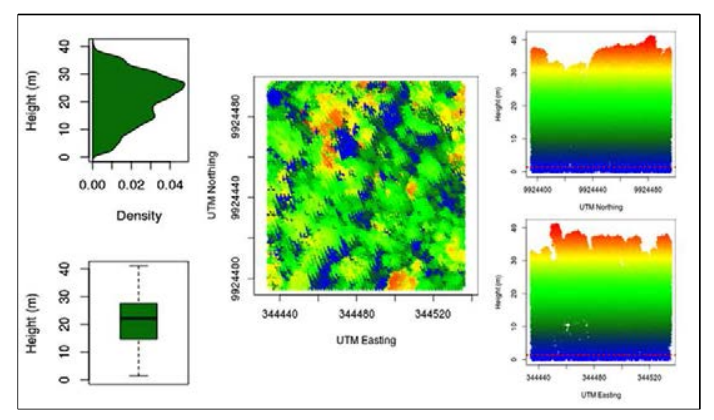

PARCELA 38

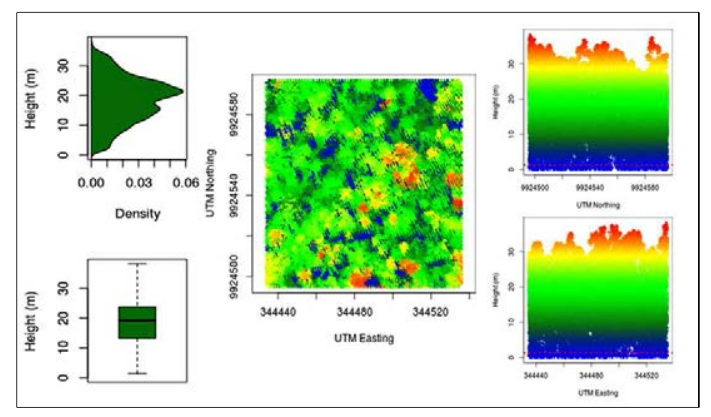

PARCELA 39

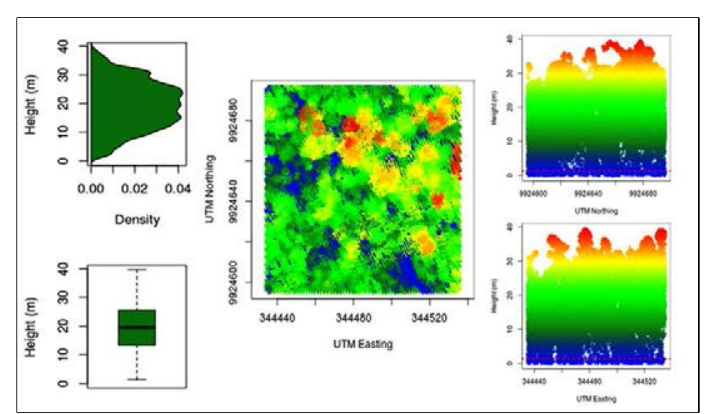

PARCELA 40 


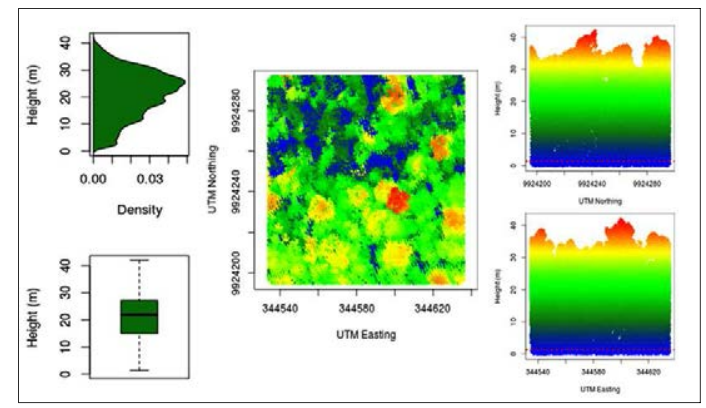

PARCELA 41

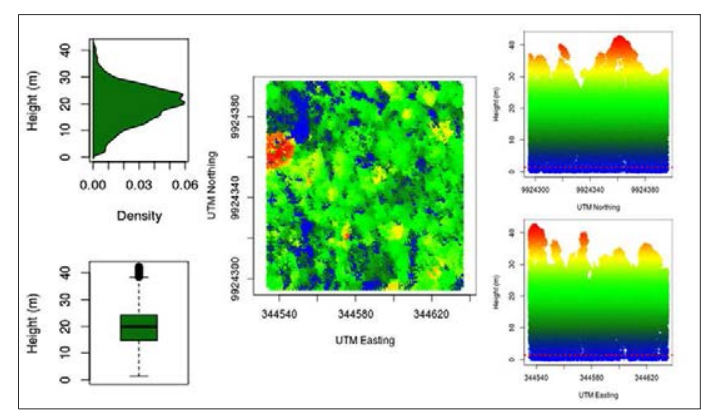

PARCELA 42

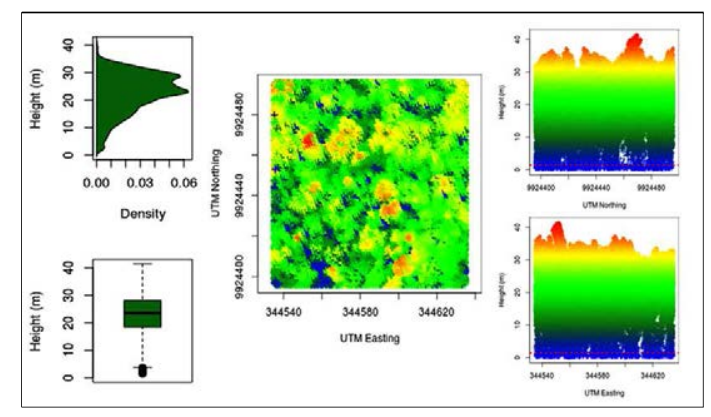

PARCELA 43

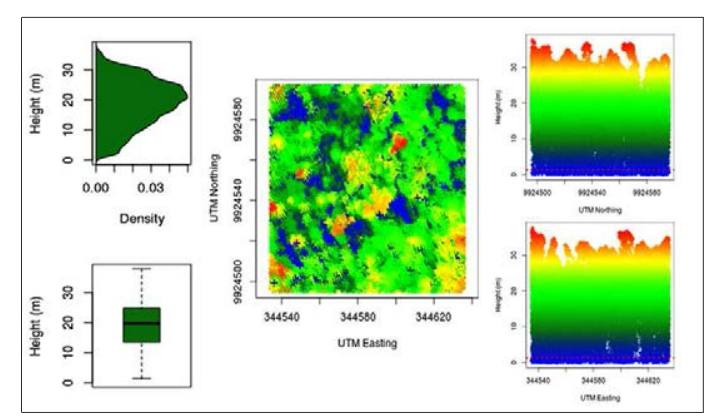

PARCELA 44

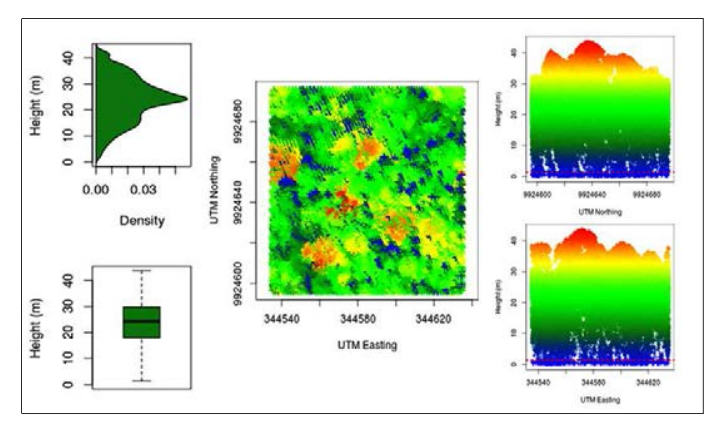

PARCELA 45

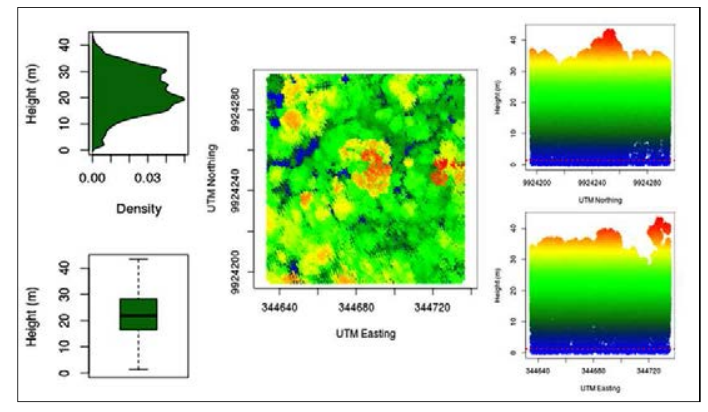

PARCELA 46

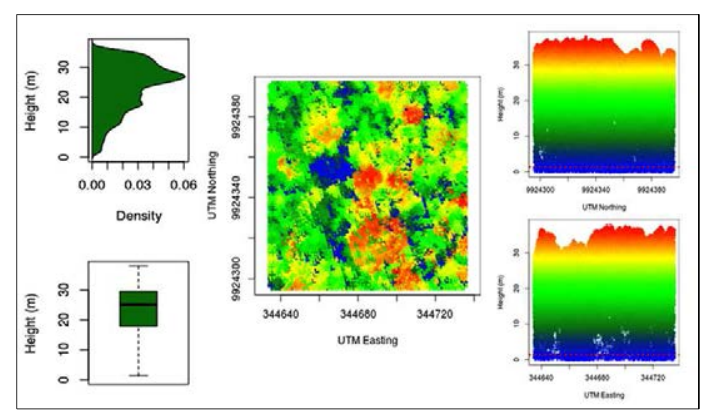

PARCELA 47

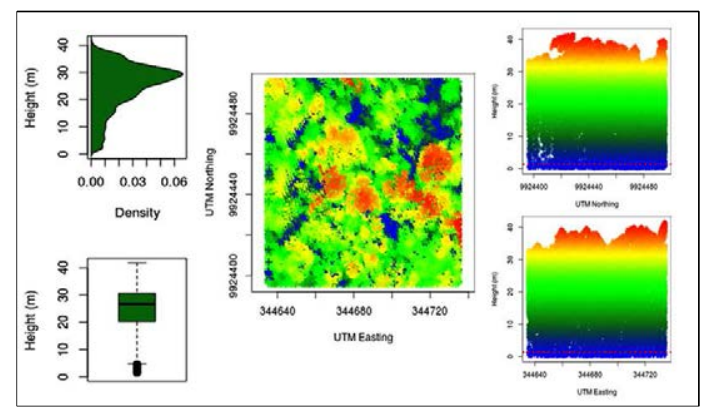

PARCELA 48

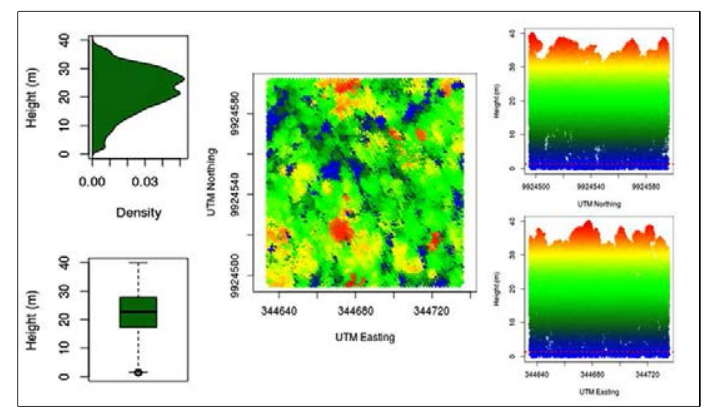

PARCELA 49

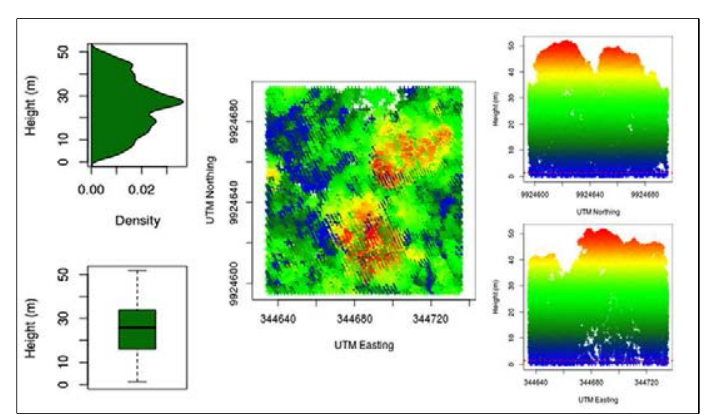

PARCELA 50 


\section{ANEXO F}

\section{Mapa de altura media al tope del dosel de la zona de estudio}




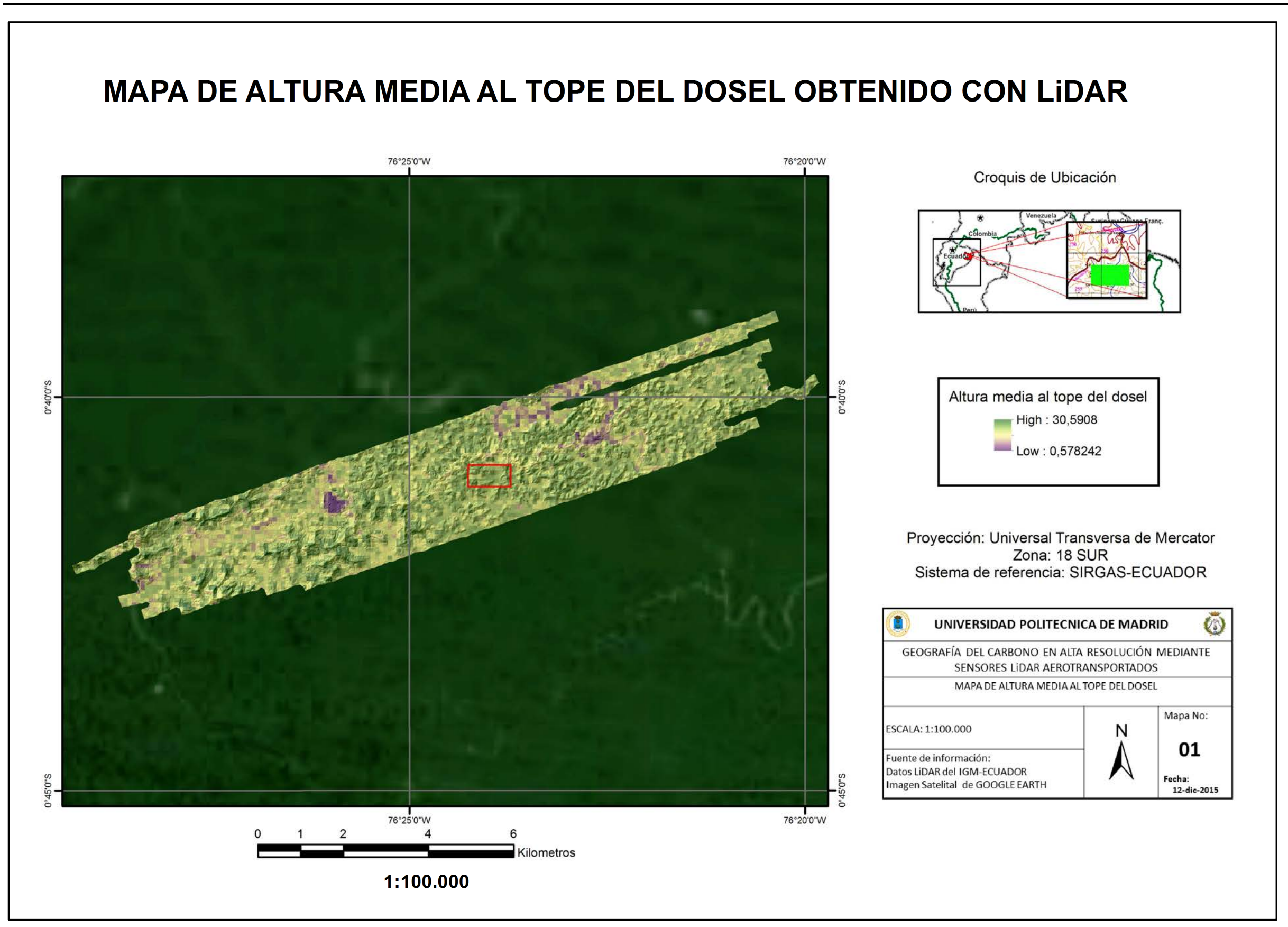




\section{ANEXO G}

\section{Mapa de las reservas de carbono sobre el suelo en la zona de estudio}


MAPA DE DENSIDAD DE CARBONO SOBRE EL SUELO OBTENIDO CON LIDAR

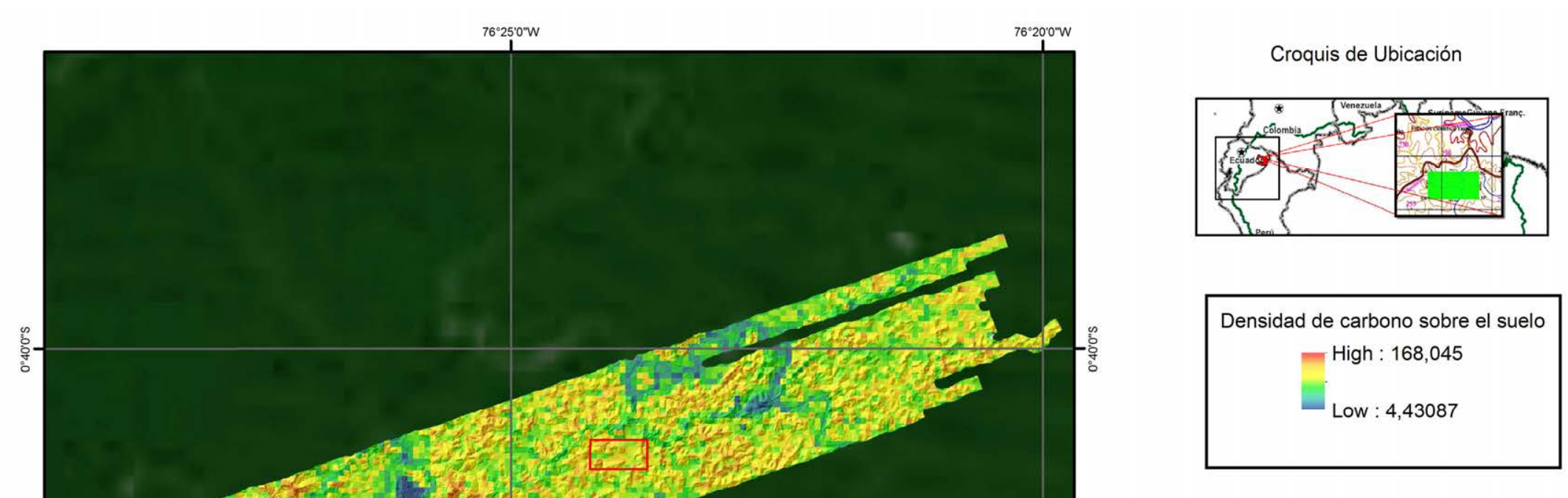

Proyección: Universal Transversa de Mercator Zona: 18 SUR

Sistema de referencia: SIRGAS-ECUADOR

\begin{tabular}{|c|c|c|}
\hline UNIVERSIDAD POLIT & DE MA & (5) \\
\hline $\begin{array}{r}\text { GEOGRAFí́A DEL CARBONO E } \\
\text { SENSORES LIDAR A }\end{array}$ & $\begin{array}{l}\text { ESOLUCI } \\
\text { ISPORTA }\end{array}$ & MEDIANTE \\
\hline MAPA DE DENSIDAD DE CARBONOS & UELO DEL & A DE ESTUDIO \\
\hline ESCALA: 1:100.000 & $\mathrm{N}$ & \\
\hline $\begin{array}{l}\text { Fuente de información: } \\
\text { Datos LiDAR del IGM-ECUADOR } \\
\text { Imagen Satelital de GOOGLE EARTH }\end{array}$ & & $\begin{array}{l}\text { Cech } \\
\text { Fecha: } \\
\text { 12-dic-2015 }\end{array}$ \\
\hline
\end{tabular}

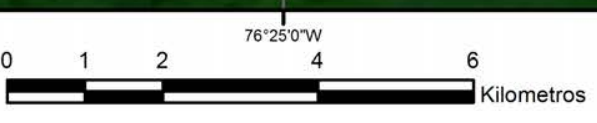

$1: 100.000$ 


\section{ANEXO H}

\section{Certificados de las estancias de investigación}


Estancia de investigación en el Dpto. de Ecología Global del Carnegie Institution for Science en la Universidad de Stanford 
Estancia de investigación en la Universidad de las Fuerzas Armadas 


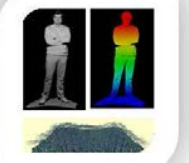

Esta tesis se terminó de escribir

el día 22 de diciembre de 2015 en la Escuela Técnica

Superior de Ingenieros en Topografía, Geodesia y Cartografía. 